\title{
Configurations and Hindered Decays of K Isomers in Deformed Nuclei with A >100
}

\author{
F.G. Kondev ${ }^{\mathrm{a}, *}$, G.D. Dracoulis ${ }^{\mathrm{b}, 1}$, T. Kibédi ${ }^{\mathrm{b}}$ \\ ${ }^{a}$ Nuclear Engineering Division, Argonne National Laboratory, Argonne, IL 60439, U.S.A. \\ ${ }^{b}$ Department of Nuclear Physics, Research School of Physics and Engineering, The Australian National University, Canberra, ACT 2601, Australia
}

\begin{abstract}
Spectroscopic information on the decay properties of high- $K$ isomers in deformed and transitional nuclei has been evaluated and collated. Assigned multi-quasiparticle configurations are included. Factors that control the transitions strengths, such as various contributions to $K$ mixing, are outlined. The systematics of $K$-forbidden transitions for different multipolarities are discussed for selected cases in terms of the hindrances, $F_{W}$, and of the reduced hindrance factor per degree of $\mathrm{K}$ forbiddenness, $f_{v}$, where $v=$ $|\Delta K-\lambda|, \Delta \mathrm{K}$ is the $\mathrm{K}$-value difference between the initial and final state and $\lambda$ is the transition multipole order. With the improved statistics for $E 1, M 1$ and $E 2$ transitions, a factorization into the product of the underlying multipolarity-dependent transition strength and a $v$-dependence, due to $K$ forbiddenness $\left(f_{0}\right)$, is possible. This suggests a weaker dependence on $K$ forbiddenness than is commonly assumed.
\end{abstract}

${ }^{*}$ Corresponding author.

Email address: E-mail: kondev@anl.gov (F.G. Kondev)

${ }^{1}$ Deceased. 


\section{Contents}

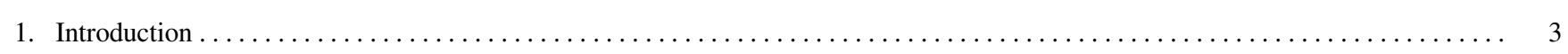

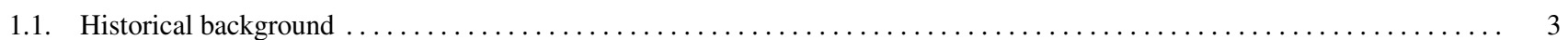

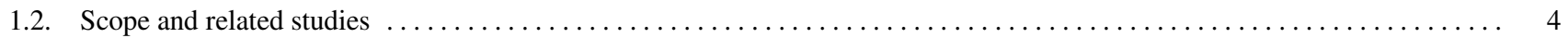

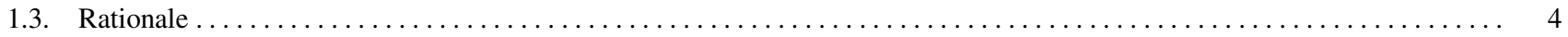

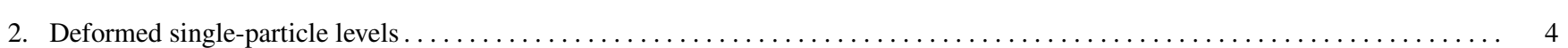

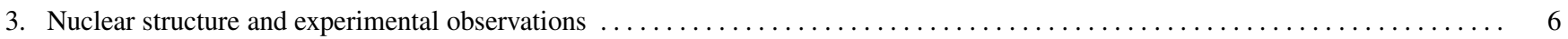

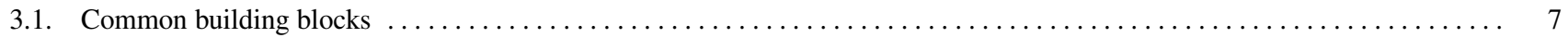

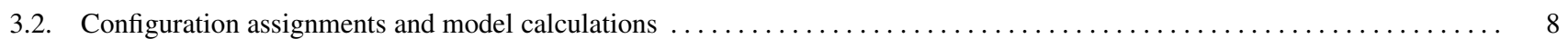

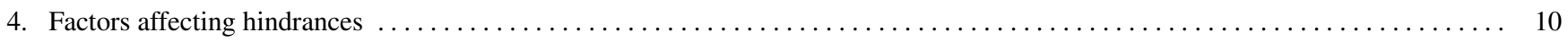

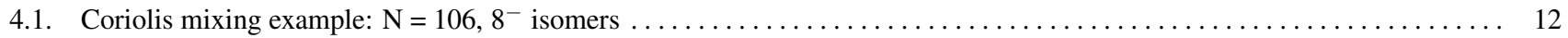

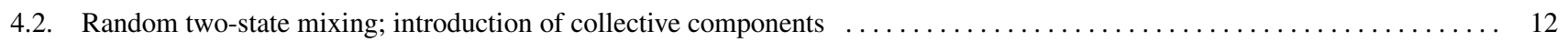

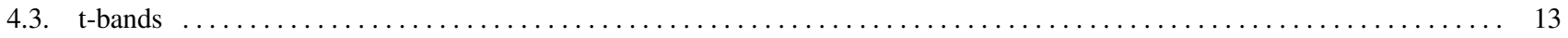

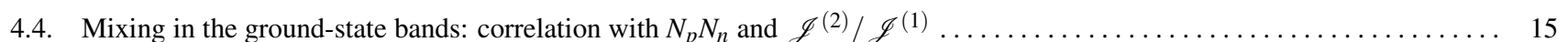

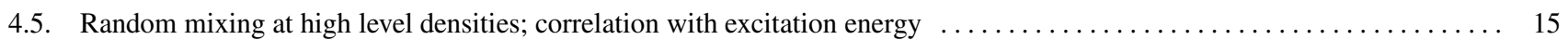

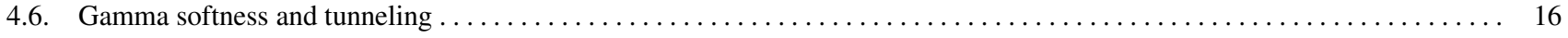

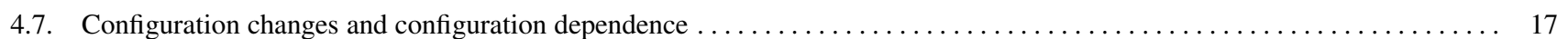

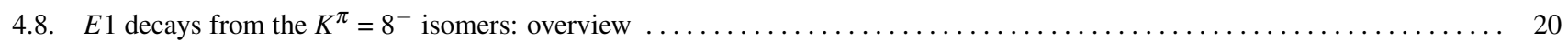

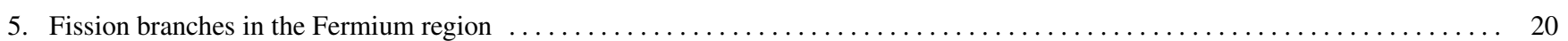

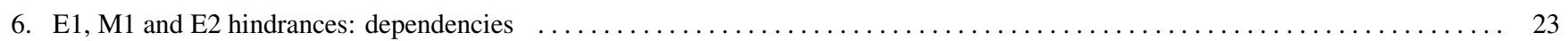

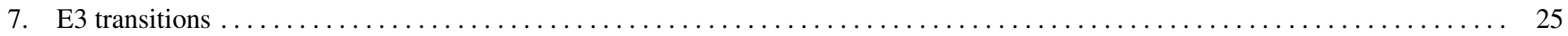

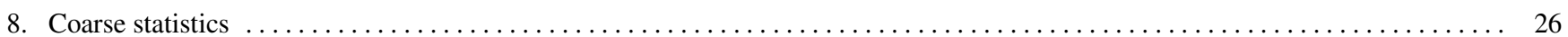

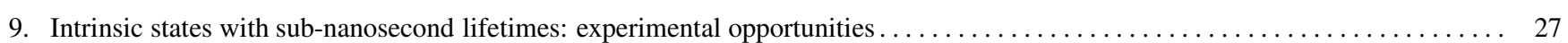

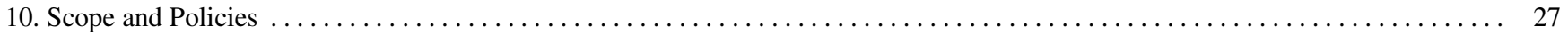

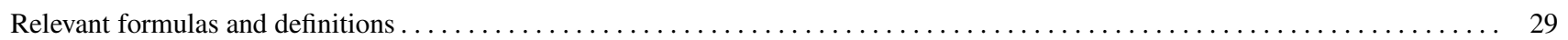

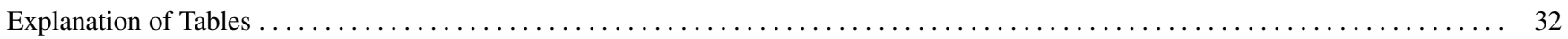

Tables

1. Spectroscopic Information and K-forbidden Transition Probabilities. $\ldots \ldots \ldots \ldots \ldots \ldots \ldots \ldots \ldots \ldots \ldots \ldots \ldots \ldots \ldots \ldots$

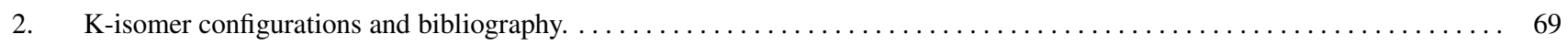




\section{Introduction}

\subsection{Historical background}

Isomers have often played a significant role in the understanding of nuclear properties, beginning with the ideas of Soddy [? ? ], the early discoveries of Hahn [? ] and the crucial theoretical insight of von Weizsäcker [? ]. In fact, von Weizsäcker showed that the combination of a large angular momentum change and low transition energy could lead to a long lifetime for electromagnetic decay. The subsequent observation of isomers in many nuclei and the ensuing attempts at interpretations, configuration assignments and quantitative analyses of the lifetimes (see, for example Refs. [? ? ? ? ? ? ]) stimulated the development of nuclear models. In effect, they provided a window into the important intrinsic states in nuclei, formed by the occupation of single-particle orbits near the proton and neutron Fermi surfaces.

Not the least of these developments was the primary evidence for the existence of deformed nuclei and the understanding that evolved from the seminal paper of Bohr and Mottelson [? ]. These authors interpreted the $\gamma$-ray cascade following the decay of a 5.5 hour isomer in ${ }^{180} \mathrm{Hf}$ [? ] as a sequence of transitions within the rotational band of an axially-deformed, even-even nucleus. The fact that the moment of inertia deduced from the state energy spacings was only about a third of that expected for rigid rotation of a nucleus with the deformation implied by the quadrupole moments [? ? ] led to the concept of pairing-induced superfluidity in nuclei [? ]. At first, the long half-life of the initial state in ${ }^{180} \mathrm{Hf}$ was assumed to arise from a transition of high multipolarity, but the interpretation changed to attribution of the hindrance to the breaking of a selection rule associated with a new quantum number, $K$, identified as the projection of the nuclear total angular momentum on the deformation axis [? ].

The early definition of an isomeric (metastable) state was that a level with a "measurable" lifetime (already in the 1950s down

to a limit of $\geq 10^{-10} \mathrm{~s}$ ). Later this drifted to mean states with lifetimes that were "directly" measurable; i.e., by electronic means, implying lifetimes of a nanosecond or more. The semantics are not important and, perhaps, a more careful definition would focus on whether the lifetimes are unusually long, implying a state of a different structure, and especially, an intrinsic state. Consider, for example, that the $2^{+}$members of rotational bands in well-deformed heavy nuclei have lifetimes of a few nanoseconds by virtue of the low energy of their $E 2$ decays, despite their large enhancement: these are not of interest in the present context. The central point is not whether the lifetimes are arbitrarily long or short, but whether the involved transition strengths that depend in detail on the underlying nuclear structure can be understood, a point emphasized many years ago by Moszkowski [? ]. Of course, from the point of view of possible applications, lifetimes and the feasibility of excitation by non-nuclear means are often of more specific interest [?].

As well as being a signature of intrinsic states, isomers provide an experimental tool that enables high-sensitivity spectroscopy, exposing not only the isomers themselves, but also the decay path of the states preceding and following them, which otherwise might be difficult to observe. Extensive studies have been carried out in the past few decades, particularly with heavy-ion induced, fusion-evaporation reactions, exploiting time-correlated techniques that allow the unambiguous placement of isomeric states and, importantly, the identification of rotational bands located above them (see section 3.2). However, such reactions are hampered by their limitations in reaching neutron-rich, deformed nuclei, but developments in using deep-inelastic reactions with heavy beams and relativistic fragmentation have seen a resurgence in isomeric studies. Those new approaches extended considerably the information on more neutron-rich, deformed systems, as reviewed recently [? ]. 


\subsection{Scope and related studies}

The focus of the present review is on isomers with seniority (the number of non-paired particles) equal to two and higher in even-even nuclei, three and higher in odd- $A$ nuclei, and greater than two in odd-odd nuclei. Although they are indirectly relevant, $K$-forbidden, one-quasiparticle decays in odd-A nuclei or two-quasiparticle isomers in odd-odd nuclei are, therefore, not included in the present tables.

There are a number of existing compilations with information on intrinsic structures in deformed nuclei that we list, here, for reference. They include early compilations of transition strengths in odd-A nuclei [? ], odd-odd nuclei [? ] and a range of odd-A, odd-odd and even-even isotopes [? ]. Later studies, that incidentally do not examine the decay properties in detail, include the early work of Jain et al. [? ] on intrinsic states and their configurations in deformed nuclei in the range $60<Z<76$ and $90<$ $N<114$; a series of studies [? ? ? ? ] covering configurations, intrinsic states and associated rotational bands in even-even and odd-odd, medium-heavy and heavy nuclei; and a recent compilation by Singh et al. [? ] focussed primarily on the properties of three-quasiparticle rotational bands and configurations in the $A=153-187$ region. Note also that, in the recent NUBASE2012 compilation [? ], associated with the 2012 Atomic Mass Evaluation [? ], excited isomeric states were included together with the nuclear ground states, although there are some differences in the coverage.

The identification of high- $K$ isomeric states as a means of probing the position of specific orbitals near the Fermi surface, of testing competing mean-field models of superheavy nuclei, and of investigating correlations, such as pairing, has been the subject of considerable recent activity [? ? ? ? ? ? ]. However, challenges remain in characterizing fully such states and their inhibited decays.

In investigations of properties of superheavy nuclei, the population of isomers, rather than the nuclear ground states, can complicate the interpretation of the corresponding $\alpha$-decay chains. This has been recently emphasized in theoretical calculations [? ], but in fact it was first raised much earlier [? ] (see Sect. 5).

\subsection{Rationale}

Given the extensive background and recent development in the study of isomers and, in this particular case, of $K$ isomers, it seemed appropriate to review the numerous nuclear structure physics aspects that govern their formation and properties. These are predominantly, but not exclusively, effects that will shorten the lifetimes by introducing allowed transitions in the context of otherwise nominally forbidden transitions. When using hindrances as a tool in nuclear spectroscopy, it is important to appreciate that a number of factors can contribute, sometimes individually, sometimes in concert, in altering the properties and, thus, in confusing the interpretation.

\section{Deformed single-particle levels}

The proton and neutron Nilsson levels of importance for the formation of prolate-deformed $K$ isomers are given in Figs. 1, 2 and 3. These were calculated using the Woods-Saxon potential with the so-called "universal" parametrization [? ] and $\beta_{4}=\beta_{2}^{2} / 6$, with $\beta_{6}=0$. Essentially three regions can be clearly separated: the lighter deformed nuclei (mass $\sim 100-150$ ), the mass $170-190$ region, which extends at its borders into the transitional nuclei, and the heavy nuclei with mass $\sim 250$.

The important orbitals are contained in the boxes in each figure. These are, for the $A=100-150$ region: the $5 / 2^{+}[422], 5 / 2^{-}[303]$ and $7 / 2^{+}[413]$ protons, and the $5 / 2^{+}[402], 5 / 2^{+}[413], 5 / 2^{-}[532], 7 / 2^{-}[523], 7 / 2^{+}$[404], and $9 / 2^{-}[514]$ neutrons; 

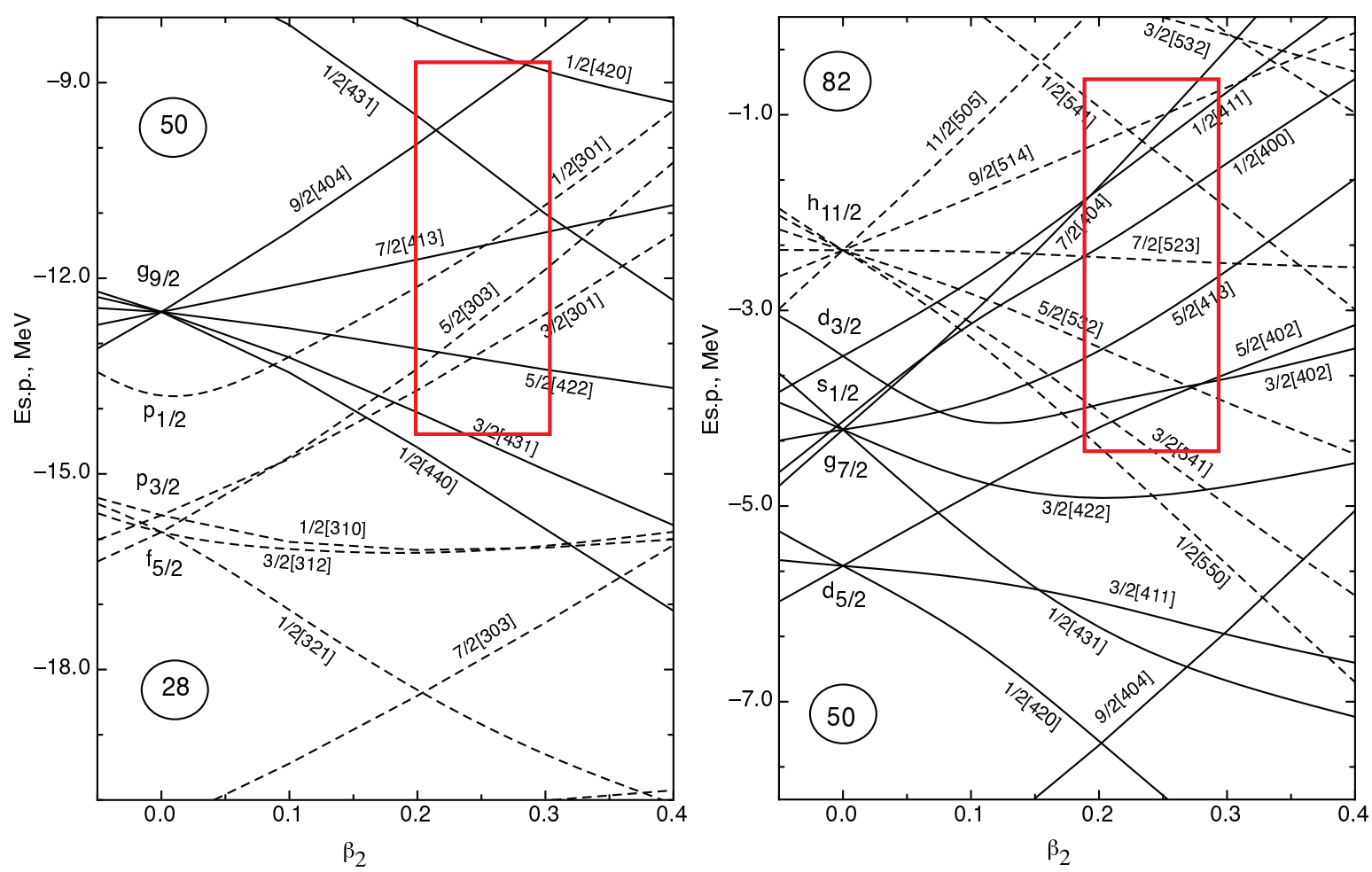

Fig. 1: Nilsson levels for protons (left) and neutrons (right) in the $A \sim 100-150$ region. Boxes indicate the main orbitals of interest.
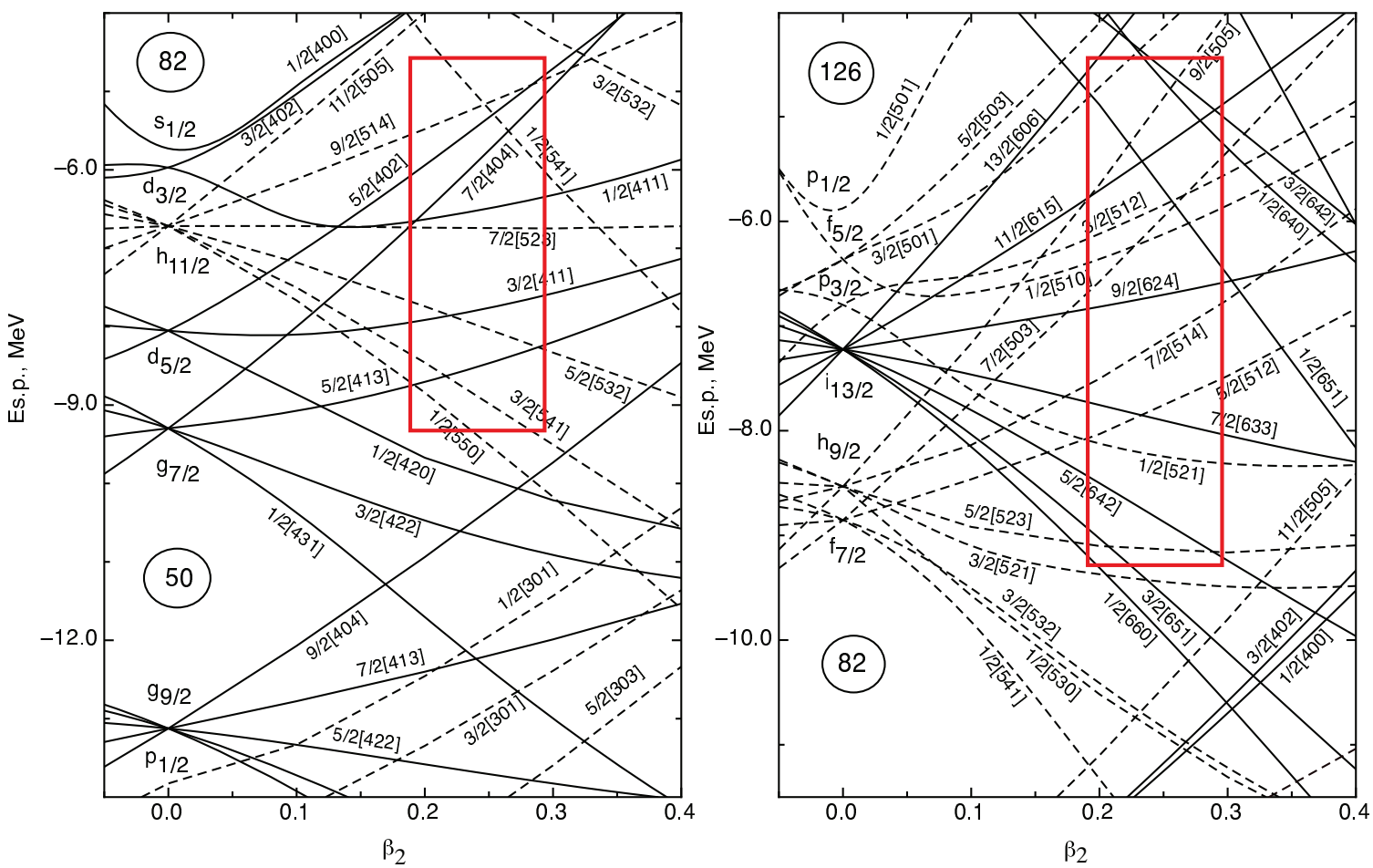

Fig. 2: Nilsson levels for protons (left) and neutrons (right) in the $A \sim 170-190$ region. Boxes indicate the main orbitals of interest. 

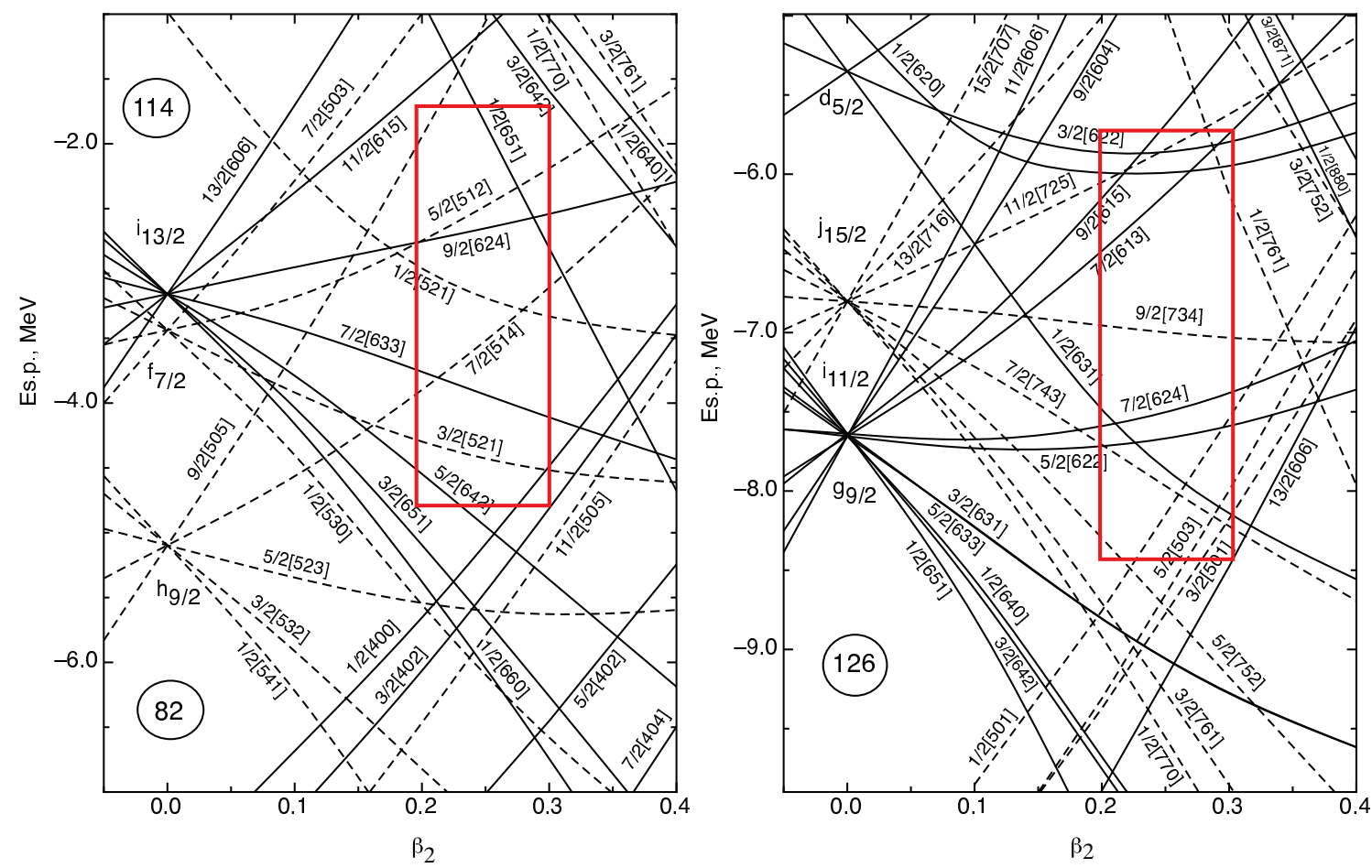

Fig. 3: Nilsson levels for protons (left) and neutrons (right) in the $A \sim 250$ region. Boxes indicate the main orbitals of interest.

for the $A=170-190$ region:

the $5 / 2^{+}[402], 5 / 2^{+}[413], 5 / 2^{-}[532], 7 / 2^{-}[523], 7 / 2^{+}$[404], and $9 / 2^{-}$[514] protons,

and the $5 / 2^{-}[512], 7 / 2^{+}[633], 7 / 2^{-}[514], 7 / 2^{-}[503], 9 / 2^{+}[624]$, and $11 / 2^{+}[615]$ neutrons;

and, finally, for the $A=250$ region:

the $5 / 2^{-}$[512], 7/2 ${ }^{+}$[633], 7/2- [514], and $9 / 2^{+}$[624] protons,

and the $5 / 2^{+}[622], 7 / 2^{+}[624], 7 / 2^{+}[613]$, and $9 / 2^{-}[734]$ neutrons.

Note that complementary orbitals appear in different regions. For example, the $7 / 2^{+}[404]$ and $9 / 2^{-}[514]$ neutrons in the $A=100-150$ region match the $7 / 2^{+}[404]$ and $9 / 2^{-}[514]$ protons in the $A=170-190$ region. Similarly, the $7 / 2^{-}[514]$ and $9 / 2^{+}[624]$ neutrons in the $A=170-190$ region are matched by the 7/2- [514] and 9/2 ${ }^{+}$[624] proton orbitals in the $A \sim 250$ region.

\section{Nuclear structure and experimental observations}

It should be remembered that isomeric states in deformed nuclei, as observed in the laboratory, are not bare intrinsic states. Deformation implies that the wave function will include a rotational component, so that an isomer cannot be viewed separately from the rotational band of which it forms the bandhead. Indeed, the band properties are sensitive to the components making up the configuration and can be used to test the proposed configuration [? ]. Furthermore, rotation inevitably implies some level of $K$ mixing. Consequently, in terms of spectroscopic and nuclear structure information, the preferred experimental situation is one where both (isomeric) bandheads and the associated collective structures can be identified. This occurs, in general, when the multi-quasiparticle states compete with collective levels based on lower-energy intrinsic states of less complexity, and when the intrinsic states are isomers, facilitating the temporal ordering of specific sequences. For both bandheads and their associated 
rotational states to be populated, they need to be near the yrast line, a situation that prevails for a number of nuclei close to stability in the $Z=70-76$ region. From the present compilation, about $70 \%$ of the isomers are found to have associated rotational bands, as indicated in Table 2

\subsection{Common building blocks}

Particular combinations of Nilsson orbitals, which can be viewed as simple building blocks for the formation of more complex multi-quasiparticle states, occur regularly in the A $\sim 180$ region. Common two-quasineutron $(v)$ and two-quasiproton $(\pi)$ ones include:

(i) $6^{+}: \pi 5 / 2^{+}[402], 7 / 2^{+}[404]$,

(ii) $6^{+}: v 5 / 2^{-}[512], 7 / 2^{-}[514]$,

(iii) $8^{-}: \pi 7 / 2^{+}[404], 9 / 2^{-}[514]$,

(iv) $8^{-}: v 7 / 2^{-}[514], 9 / 2^{+}[624]$.

As indicated earlier, in both sets there are alternative neutron and proton pairs. The two-proton, $6^{+}$state is observed at similar excitation energies in the well-deformed $\mathrm{Hf}$ isotopes (Fig. (4) with a lifetime in the nanosecond range, except in ${ }^{176} \mathrm{Hf}$ where its lower energy of $1333 \mathrm{keV}$ results in a much longer lifetime. The $1333-\mathrm{keV}$ state is, in fact, strongly mixed, with a wave function given by $61 \% v^{2}+39 \% \pi^{2}[?]$. For the $8^{-}$isomers in the $N=106$ isotones, shown in Fig. 4 the two-neutron configuration dominates, except in the case of ${ }^{178} \mathrm{Hf}$ where significant mixing occurs between the neutron and proton $8^{-}$alternatives, with the result that the lower experimental state is strongly mixed, being about $64 \% v^{2}+36 \% \pi^{2}\left[? ? ?\right.$ ? ]. However, the lifetimes of the $8^{-}$ states range from seconds in the lower- $Z$ cases to about a microsecond in ${ }^{188} \mathrm{~Pb}$, where the isomer exists in a deformed secondary minimum, while the ground state is spherical [? ]. (The factors which control the lifetimes will be outlined in more detail below.)

As can be seen in Table 2, the lowest 3-quasiparticle states in the odd-neutron isotopes neighboring ${ }^{178} \mathrm{Hf}$ arise from the coupling of the $7 / 2^{-}[514]$ or $9 / 2^{+}[624]$ neutrons to the two-proton $6^{+}$or $8^{-}$configurations giving an expectation of $K^{\pi}=19 / 2^{-}, 23 / 2^{+}$and $25 / 2^{-}$states (as seen in ${ }^{177} \mathrm{Hf}$, for example). The complementary situation occurs in the odd-proton isotopes ${ }^{177} \mathrm{Lu}$ and ${ }^{179} \mathrm{Ta}$, where the low-lying $7 / 2^{+}[404]$ and $9 / 2^{-}[514]$ protons couple to the two-neutron $8^{-}$cores. Higher seniority states are generally comprised of the same building blocks, with a preference for "balanced" configurations involving similar numbers of broken neutron and proton pairs, controlled partly by the fact that the pairing energy $\Delta_{n}<\Delta_{p}$. For example, the combination of the $v^{2}$ and $\pi^{2} 8^{-}$ configurations leads to the low-lying $K^{\pi}=16^{+}, T_{1 / 2}=31 \mathrm{y}$ isomer in ${ }^{178} \mathrm{Hf}$, whose energy is only a little below that of the simple sum of the energies of the two $8^{-}$component states, owing to attractive residual interactions. A situation somewhat complementary to that observed in the mass-180 region occurs near mass-130, where the same Nilsson configurations occur near the neutron, rather than the proton Fermi surface. Hence, the formation of $K^{\pi}=8^{-}$isomers occurs from the $v 7 / 2^{+}[404], 9 / 2^{-}[514]$ configuration. In very heavy deformed nuclei, similar low-lying $K^{\pi}=8^{-}$states can be formed by the $\pi 7 / 2^{-}[514], 9 / 2^{+}[624]$ and either of the $v 7 / 2^{+}[624], 9 / 2^{-}[734]$ or $v 7 / 2^{+}[613], 9 / 2^{-}[734]$ configurations. This leads to a situation resembling that in ${ }^{178} \mathrm{Hf}$, where low-lying $K^{\pi}=16^{+}$states, (several in this case) are predicted. Multi-quasiparticle calculations give similar results for the No $(Z=102)$ and $\mathrm{Rf}$ $(Z=104)$ chain of even-even isotopes [? ]. Two $8^{-}$alternatives lie in close proximity and low-lying $(\sim 2.5 \mathrm{MeV}) \mathrm{K}^{\pi}=16^{+}$states are expected, as shown in Fig. 5. The predicted $16^{+}$states have yet to be observed, but the situation will be different from that of

${ }^{178} \mathrm{Hf}$, since fission is likely to be the dominant decay mode, presenting a considerable experimental challenge. Similar predictions 


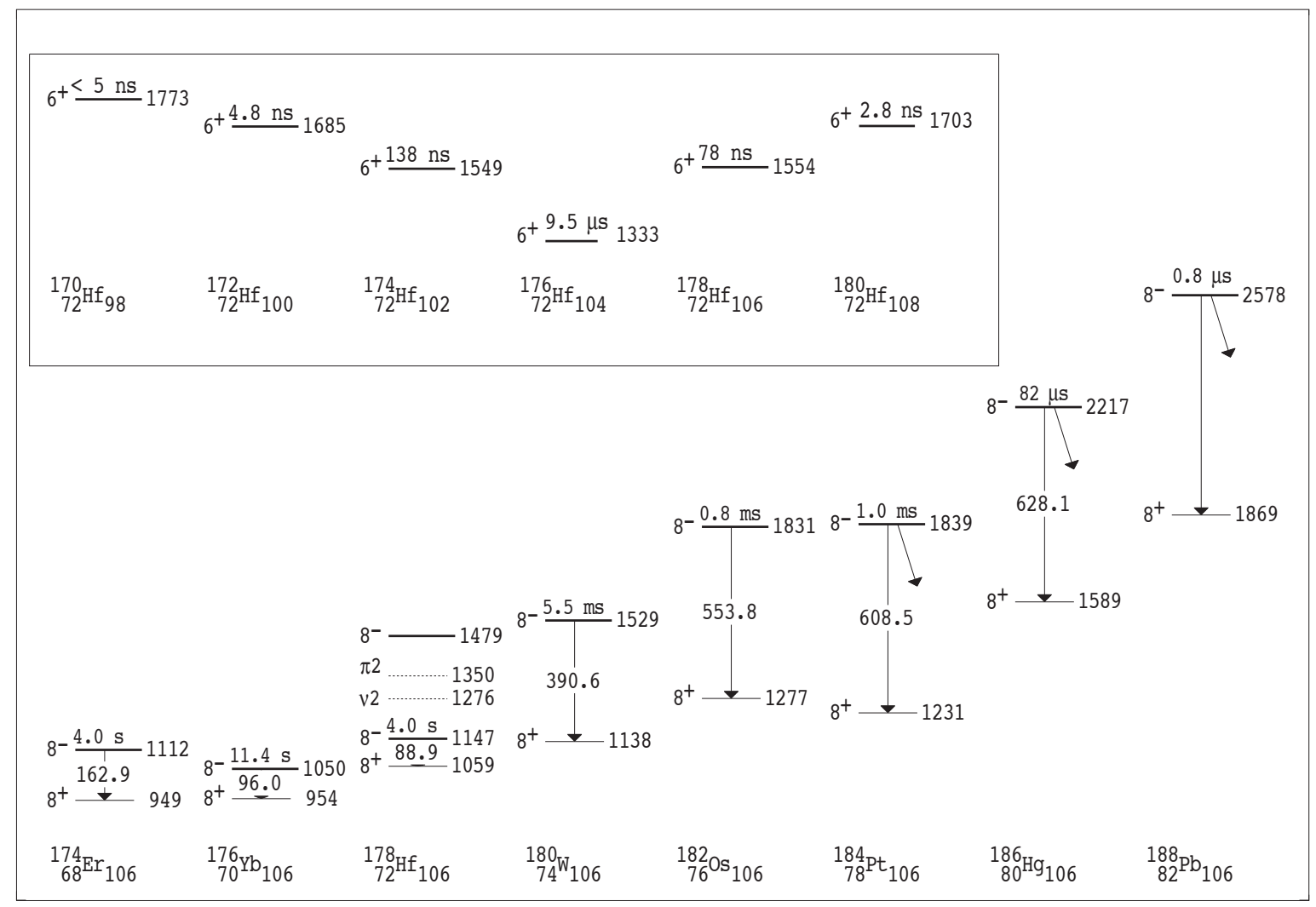

Fig. 4: Systematics of $K^{\pi}=6^{+}$isomers in the $Z=72$ (Hf) isotopes and the $K^{\pi}=8^{-}$isomers in the $N=106$ isotones.

have been made for ${ }^{250,252,254}$ No by Liu et al. [? ] and an even more extreme case is proposed to occur in ${ }^{270}$ Ds, where a lowlying $20^{+}$state could be produced by a combination of the favored $K^{\pi}=10^{-}, v 9 / 2^{+}[615], 11 / 2^{-}[725]$ and $\pi 9 / 2^{-}[505], 11 / 2^{+}[615]$ building blocks. Candidates for the $10^{-}$components are known experimentally [? ? ], but whether states as high as $K^{\pi}=20^{+}$could be formed experimentally remains a open question.

\subsection{Configuration assignments and model calculations}

Model calculations relevant to the $A \sim 180$ region include the early studies of multi-quasiparticle states by Åberg [? ] and, more generally, by Andersson et al [? ], while a broad range of isomers and assigned configurations were reviewed by de Voigt $e t$ al. [? ]. In recent years, a large number of comparisons with experiment as well as predictions for neutron-rich cases have been reported (see, for example Ref. [?]), based largely on multi-quasiparticle (fixed deformation) models that include self-consistent pairing/blocking, and either a Nilsson or Woods-Saxon basis for the initial single-particle states, as detailed in Refs. [? ? ? ]. The models differ largely in their degree of sophistication in the treatment of pairing and blocking. While they allow to identify which configurations will lead to relatively low-lying, high- $K$ states, they cannot by themselves predict the decay properties of isomers.

The assumption of fixed deformations is reasonable in the middle of the deformed region, but calculations that take into account the configuration-dependence of deformation produce somewhat different expectations in the more neutron-rich cases, where the nuclei tend to become softer (as $N=116$ is approached, for example). Nevertheless, the deformations still tend to be stabilized by particle excitations [?]. 


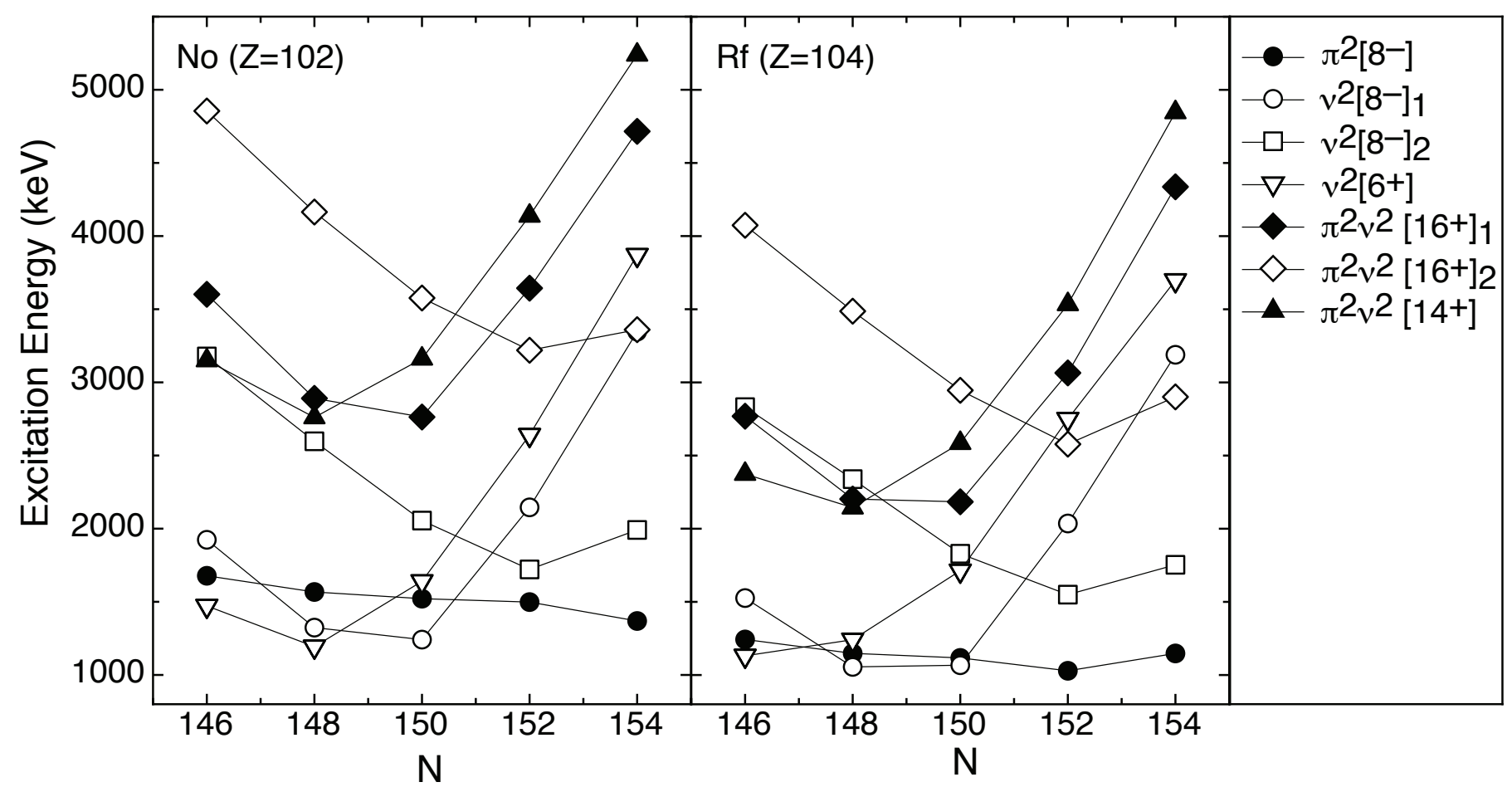

Fig. 5: Predicted two- and four-quasiparticle states in No and Rf isotopes. (Results from Kondev et al. [? ].) Configurations: $\pi^{2}\left[8^{-}\right]: \pi 7 / 2^{-}[514], 9 / 2^{+}[624]$; $v^{2}\left[8^{-}\right]_{1}: v 7 / 2^{+}[624], 9 / 2^{-}[734] ; v^{2}\left[8^{-}\right]_{2}: v 7 / 2^{+}[613], 9 / 2^{-}[734] ; v^{2}\left[6^{+}\right]: v 5 / 2^{+}[622], 7 / 2^{+}[624] ; \pi^{2} v^{2}\left[14^{+}\right]: v 5 / 2^{+}[622], 7 / 2^{+}[624] \otimes \pi 7 / 2^{-}[514], 9 / 2^{+}[624] ;$ $\pi^{2} v^{2}\left[16^{+}\right]_{1}: v 7 / 2^{+}[624], 9 / 2^{-}[734] \otimes \pi 7 / 2^{-}[514], 9 / 2^{+}[624] ; \pi^{2} v^{2}\left[16^{+}\right]_{2}: v 7 / 2^{+}[613], 9 / 2^{-}[734] \otimes$

$\pi 7 / 2^{-}[514], 9 / 2^{+}[624]$.

Obviously, configuration assignments lie at the heart of these studies. The factors which are commonly used to propose assignments include:

1. The spin and parity $J^{\pi}$ of the isomer: in some cases only a particular combination of orbitals can reasonably result in the desired long-lived state with the specific spin and parity at a low energy.

2. Decay properties, such as strengths and/or hindrances, that might be part of the considerations leading to the spin and parity assignments. This depends on the initial and final state configurations for each transition, including the initial and final $K$ value.

3. The rotational band properties such as (i) alignments, which depend on the properties of the protons and neutrons making up the configuration, and on the pairing, which is both seniority-dependent and configuration-dependent (i.e., specifically which particles are involved) and (ii) the in-band properties, principally the cascade and crossover $\gamma$-ray branching ratios from which configuration-dependent properties such as the $\left(g_{K}-g_{R}\right) / Q_{0}$ ratio can be deduced. (See Ref. [? ] for an evaluation of the rotational properties and of the role of specific orbitals for a large number of related multi-quasiparticle states.)

4. Guidance from multi-quasiparticle calculations such as those outlined earlier, and consideration of empirical quantities such as the excitation energies of lower-seniority components that occur frequently in the same or neighboring nuclei, the so-called "building blocks" [? ].

Clearly, in assigning configurations there is considerable value in identifying the associated rotational bands (sometimes difficult 


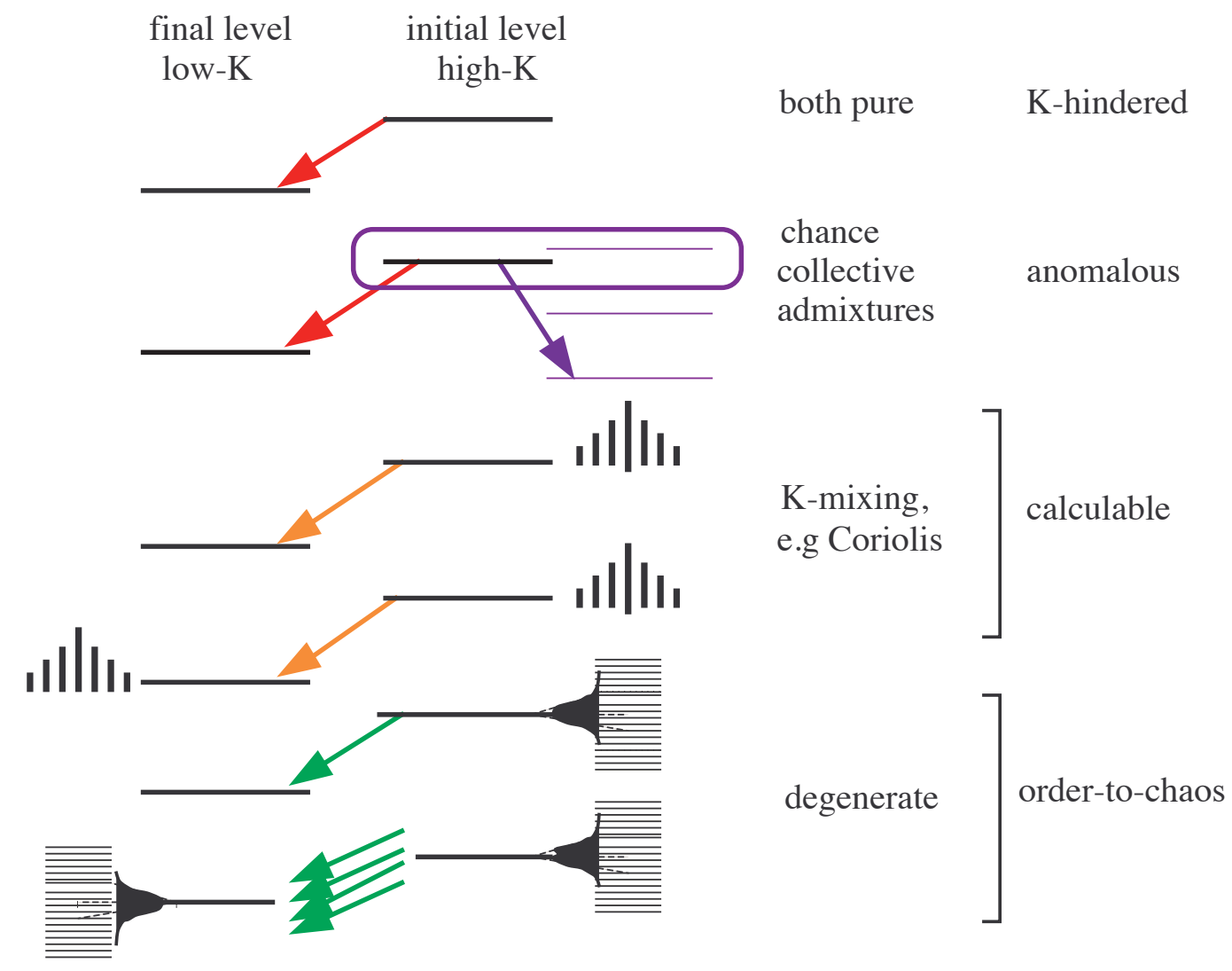

Fig. 6: A view of the hierarchy of $K$-admixtures, progressing from pure states (top) to a completely mixed situation (bottom).

to do directly for particularly long-lived states). Furthermore, most of the factors above are inter-related. Therefore, all need to be considered together to achieve a consistent assignment.

\section{Factors affecting hindrances}

Long lifetimes occur when low-energy and/or high-multipolarity transitions are involved [? ], and also when $K$ conservation is challenged. When the $\gamma$-ray multipolarity, $\lambda$, fails to match the difference in $K$ between the initial and final states, the shortfall, known as the forbiddenness, is given by $v=\Delta K-\lambda$. However, neither the presence or absence of isomers, nor the magnitude of individual lifetimes by themselves, are necessarily a measure of the conservation of the $K$ quantum number. The magnitude of the transition inhibition needs to be examined. The hindrance $\left(F_{W}\right)$ is given by the inverse of the transition strength, or equivalently, the ratio of the partial $\gamma$-ray lifetime $\left(\tau_{\gamma}\right)$ compared to the Weisskopf estimate $\left(\tau_{W}\right)$, so that $F_{W}=\frac{\tau_{\gamma}}{\tau_{W}}$.

Early work on hindrances by Gallagher [? ] and Rusinov [? ] was followed by Löbner [? ? ] who confirmed that the observed hindrances roughly scale with the forbiddenness with a dependence that implies that the reduced hindrance $f_{V}=F_{W}^{\frac{1}{v}}$ is approximately a constant of magnitude $\sim 100$. This has formed the basis of much of the subsequent discussion of $K$ hindrances: values in the range 30 - 200 are now seen as unexceptional, although there are large variations. However, Löbner also made the point that the systematics were offset from the values implied by Rusinov's simpler evaluation, implying an underlying, $K$-independent hindrance that was multipolarity dependent. This point will be discussed and quantified in Sect.6 


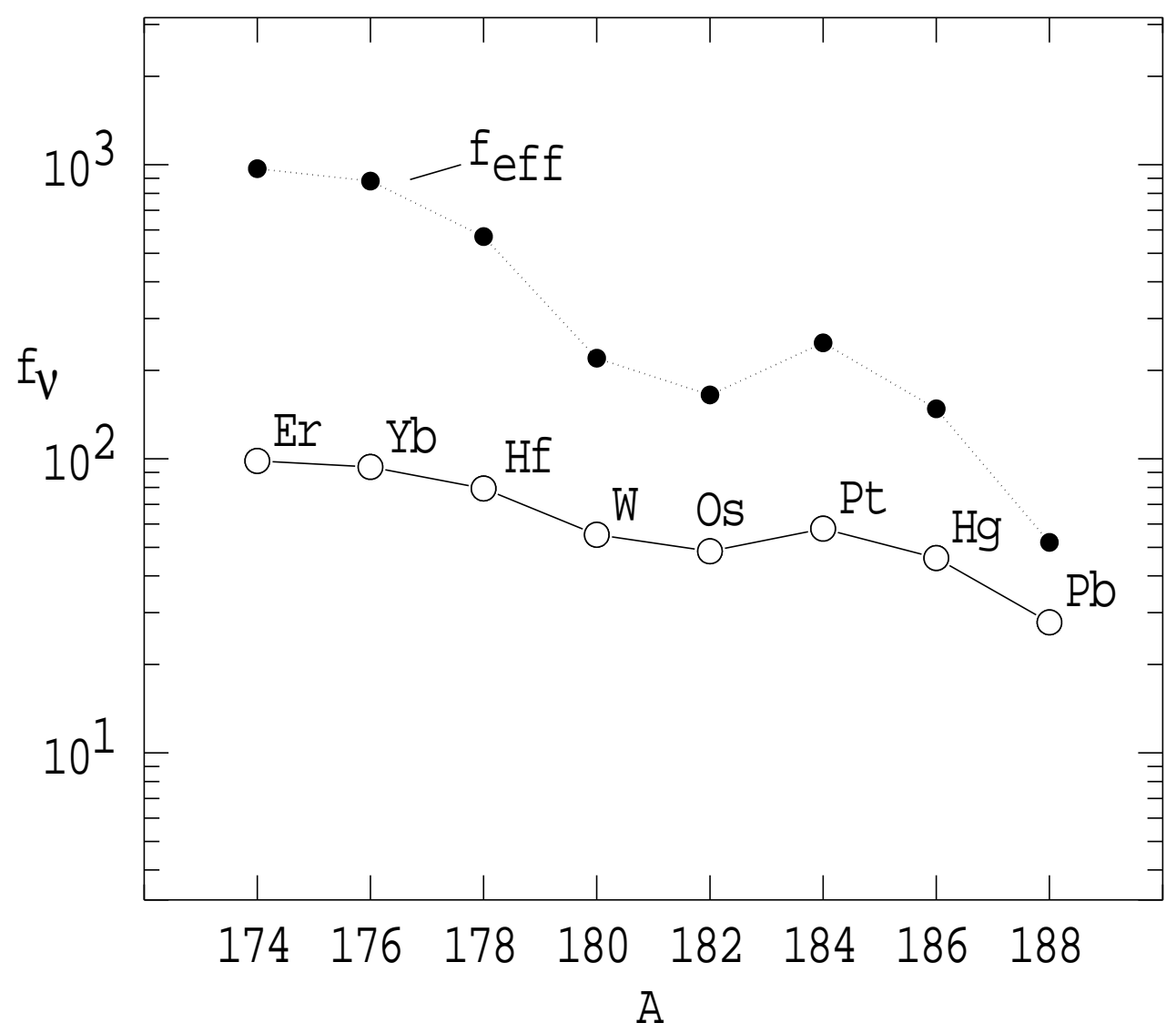

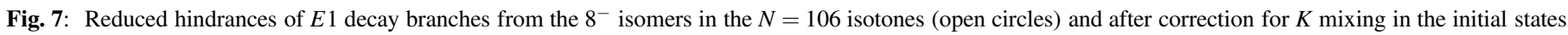
(filled circles) [? ]. Note that values for ${ }^{186} \mathrm{Hg}$ have been amended from those in Ref. [? ] given the updated experimental branches listed in Table 1.

Even approximate scaling through a single parameter is somewhat of a surprise since the transitions are in principle forbidden. They must proceed through miniscule admixtures in the tail of the wave function of either or both of the initial and final states. Such amplitudes and the resultant transition strengths would be expected to be extremely sensitive to the nuclear structure and the mixing. For these reasons, it is probably beyond the reach of current nuclear models to calculate absolute transition rates directly.

Mikhailov [?] has included modifications to the rotational model formulae for $M 1$ and $E 2$ transitions by taking into account the amplitudes of admixed components with $K$ values different from the nominal ones. These result, in effect, in allowed transitions that contribute to the total strength. The approach assumes a knowledge of the admixtures, obtained through a perturbation expansion, rather than via a specific coupling model. It can be useful in evaluating branching ratios, for example, where the amplitudes cancel. A similar formalism was presented by Bohr and Mottelson [? ]. Aside from model approaches, it is useful to classify the strengths, at least in a semi-quantitative fashion, since these form the basis for both experimental spin and parity assignments, and subsequent interpretations of the nuclear structure, including configuration assignments.

The basic issue is obviously that of $K$ mixing, which can be present, as indicated earlier, in either or both of the initial and final states. This mixing might arise from dynamical effects such as rotation or vibration, random interactions with one or more closelying states, or configuration mixing associated with the underlying nuclear potential. For instance, Coriolis coupling induced by rotation inevitably broadens the $K$ distributions, but in a relatively well-defined (calculable) fashion. One can envisage a hierarchy of mixing effects as sketched out in Fig. 6 that will be manifested in individual cases and in systematics, depending on the nuclear 
region. Examples are outlined below.

\subsection{Coriolis mixing example: $N=106,8^{-}$isomers}

The reduced hindrances (Fig. 7) of the 7-times forbidden $E 1$ decays in the $N=106,8^{-}$isomers discussed earlier are comparatively low, all having $f_{v} \leq 100$. This might be viewed as anomalous, since $E 1$ transitions (without $K$ hindrance) are usually significantly inhibited already with respect to single-particle values, with $F_{W} \sim 10^{5}-10^{6}$. (This is the argument behind the use of additional (arbitrary) reduction factors of $10^{3}$ or $10^{4}$ in some comparisons, and this can cause confusion. A more consistent approach will be discussed in Sect. 6)

The systematic fall with $Z$ seen here can be partly attributed to mixing in the final state, as discussed later, which is indirectly a function of the deformation of the $8^{+}$state of the band into which the isomer decays. The low absolute value, however, can be associated with mixing in the isomeric state due to the presence of the $9 / 2^{+}[624]$ Nilsson level, arising from the $i_{13 / 2}$ neutron, in the two-neutron configuration. To quantify this assertion, the authors of Ref. [? ] corrected the experimental values of $f_{v}$ for the $K$ admixtures in the bandhead wavefunction. These were obtained in a particle-rotor model [? ], with parameters adjusted to reproduce approximately the associated rotational band located above the isomer, allowing extraction of the underlying effective reduced hindrance $f_{e f f}$, shown in Fig. 7 . This resulted in much larger values of the reduced hindrance. As discussed in Ref. [? ], the formulation is non-linear and obviously very sensitive to the small $K$ components furthest removed from the nominal $K=8$ value, since these introduce allowed transitions into the sum of strengths.

\subsection{Random two-state mixing; introduction of collective components}

Another way that allowed components can occur and effectively overwhelm the very weak forbidden transitions is through a chance near-degeneracy between an isomer and a collective state of the same spin and parity. These occurrences are reflected in anomalously low reduced hindrances for $E 2$ transitions - with $f_{v} \leq 10$ - listed in Table 1 for various $\mathrm{Tm}, \mathrm{Yb}, \mathrm{Hf}, \mathrm{Lu}, \mathrm{Ta}$ and $\mathrm{Re}$ isotopes.

The key characteristic, which identifies these as examples of isolated two-state mixing, is that the $E 2$ and $M 1$ decays from the isomer to lower band members have an intensity ratio that reflects the in-band crossover/cascade ratio within the band itself, which in turn depends on the intrinsic properties $\left(\right.$ e.g. $\left.\left(g_{K}-g_{R}\right) / Q_{0}\right)$ ratio) of the band [? ? ]. Numerous cases have been identified, analyzed and explained within this scenario [? ? ? ? ? ? ? ? ? ]. In addition, the branch intensities were found to be very sensitive to the interaction matrix elements, which are a function of the difference in $K$ between the isomer and the state belonging to the interfering band [? ]. Values as low as a few $\mathrm{eV}$ in magnitude have been extracted in this way. (Approximate reproduction of the very small matrix element observed in ${ }^{171} \mathrm{Tm}$ has been achieved recently with the projected shell model [? ].) The decays from the 1515-keV, four-quasiparticle, $K^{\pi}=12^{+}, 450-\mathrm{ns}$ isomer in ${ }^{176} \mathrm{Lu}$ provide an example of how the fast transitions stand out as being anomalous. The reduced hindrances for its numerous branches, of different multipolarity, are given in Fig. 8 8 a). Most fall within the range of $f_{v}=30-300$ (with significant variations) except for the $1127-\mathrm{keV}$, twice-forbidden, $E 3$ transition, which is marginal at $f_{v}=12(3)$, and the six-times forbidden $355-\mathrm{keV}, E 2$ transition to the $10^{+}$member of a $K^{\pi}=4^{+}$band: its low value of $f_{v}=3.7(2)$ has been explained by two-state mixing between the isomer and the $12^{+}$member of the $K^{\pi}=4^{+}$band, which lies only $6 \mathrm{keV}$ higher. (This can only be the case, of course, if the states have the same spin and parity, which turns out to be a useful tool in assignments). An interaction matrix element of $5 \mathrm{eV}$ was sufficient to produce this observation [? ].

The assigned $v 7 / 2^{-}[514], 1 / 2^{-}[521], 9 / 2^{+}[624] \otimes \pi 7 / 2^{+}[404]$ configuration contains an $i_{13 / 2}$ neutron orbital and, in principle, could be corrected for $K$ mixing, as was discussed earlier for the $8^{-}$isomers (Sec.4.1). This is more important for the case of the 

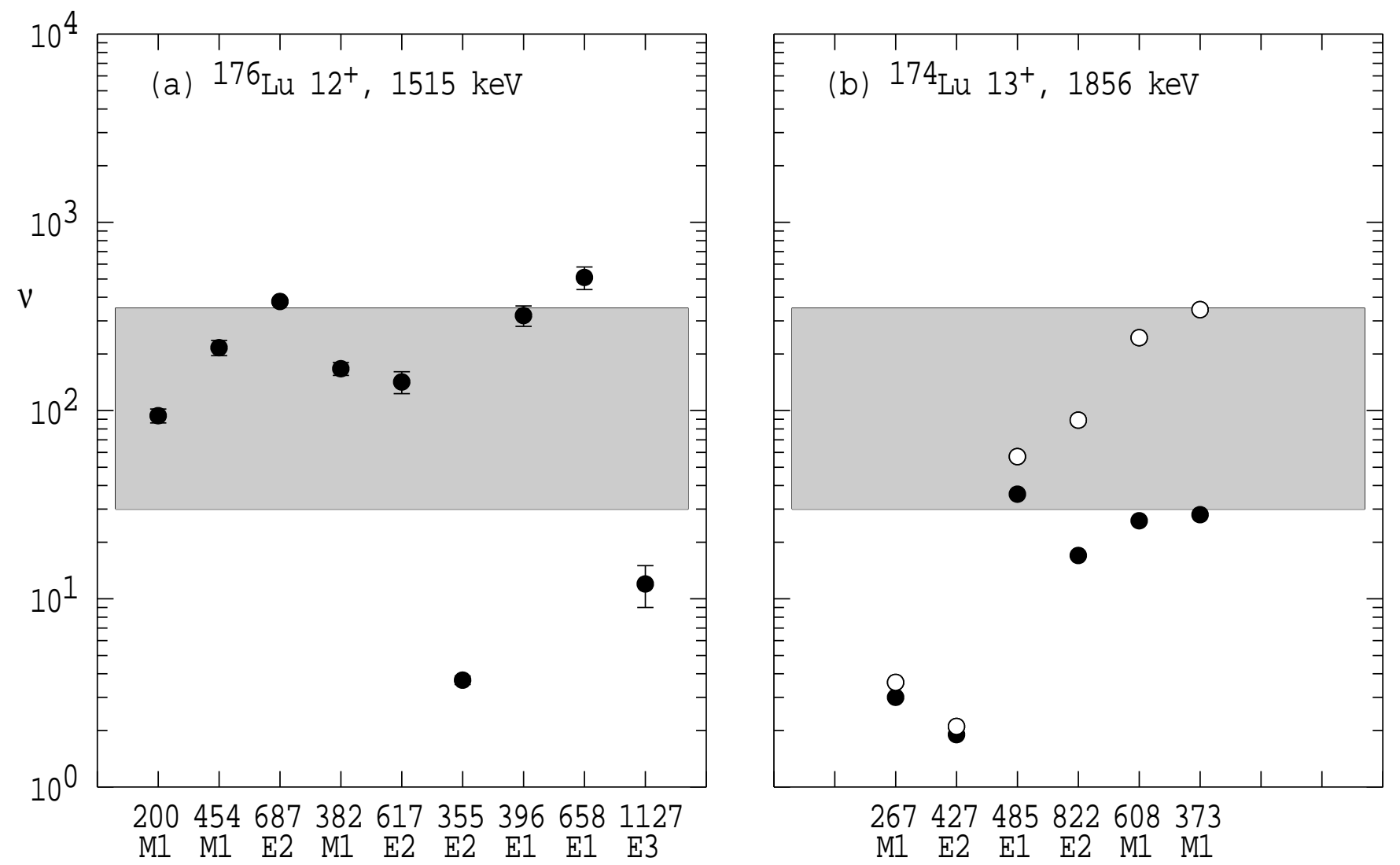

Fig. 8: Reduced hindrances for decays from (a) the $K^{\pi}=12^{+}, 1515-\mathrm{keV}$ isomer in ${ }^{176} \mathrm{Lu}\left[\right.$ ? ] and (b) the $13^{+}, 1856-\mathrm{keV}$ isomeric state in ${ }^{174} \mathrm{Lu}[$ ? ]

$K^{\pi}=13^{+}$isomer observed at $1856 \mathrm{keV}$ in ${ }^{174} \mathrm{Lu}[?]$ ]. It has a chance degeneracy with a low- $K$ rotational band member and $K$ mixing in both the initial and final states [? ]. Its $K$ mixing is also stronger because the Fermi level is lower in the $i_{13 / 2}$ neutron shell, thus enhancing Coriolis effects. The reduced hindrances of its decays are presented in Fig. 8(b). The 267- and 427-keV, $M 1$ and $E 2$ decays to the $K^{\pi}=0^{+}$band have very low values - they are essentially unhindered - all the other branches are being marginally low with $f_{v}$ values of 20-30.

In this case, the $K$ admixtures were calculated in Ref. [? ] with a simplified particle-rotor model for both the four-quasiparticle isomeric state (and its associated rotational band), which was assigned the $v 7 / 2^{-}[514], 5 / 2^{-}[512], 7 / 2^{+}[633] \otimes \pi 7 / 2^{+}[404]$ configuration, and for the family of two-quasiparticle bands arising from the coupling of the $7 / 2^{+}$[404] proton to the $i_{13 / 2}$ neutron set of orbitals, where the Fermi level is near the $7 / 2^{+}[633]$ state. The lowest of these are the $K^{\pi}=0^{+}$and $7^{+}$bands to which most of the isomeric decays proceed. The corrected reduced hindrances are given as open circles in Fig. 8 (b). The only ones which do not rise to be within the normal range are those to the $0^{+}$band since, as indicated, these are dominated by the admixture of the $12^{+}$ collective state, which induces very small amplitude collective transitions that overwhelm any forbidden components.

\section{3. t-bands}

The relatively fast decay of the $K^{\pi}=35 / 2^{-}, 3349-\mathrm{keV}, 1.0-\mu$ s isomer in ${ }^{179} \mathrm{~W}$ via a $576-\mathrm{keV}$ transition, seemingly directly to the $31 / 2^{-}$member of the $7 / 2^{-}$[514] band, was originally difficult to understand [? ]. It corresponded to a transition with $v=\Delta K-$ $\lambda=12$, and $f_{v}=2$. The problem was resolved in a subsequent detailed study [? ] which showed that the decay pattern was much more complex, largely because of mixing between the isomeric state and two other $35 / 2^{-}$states, one within the $K^{\pi}=7 / 2^{-}$band 

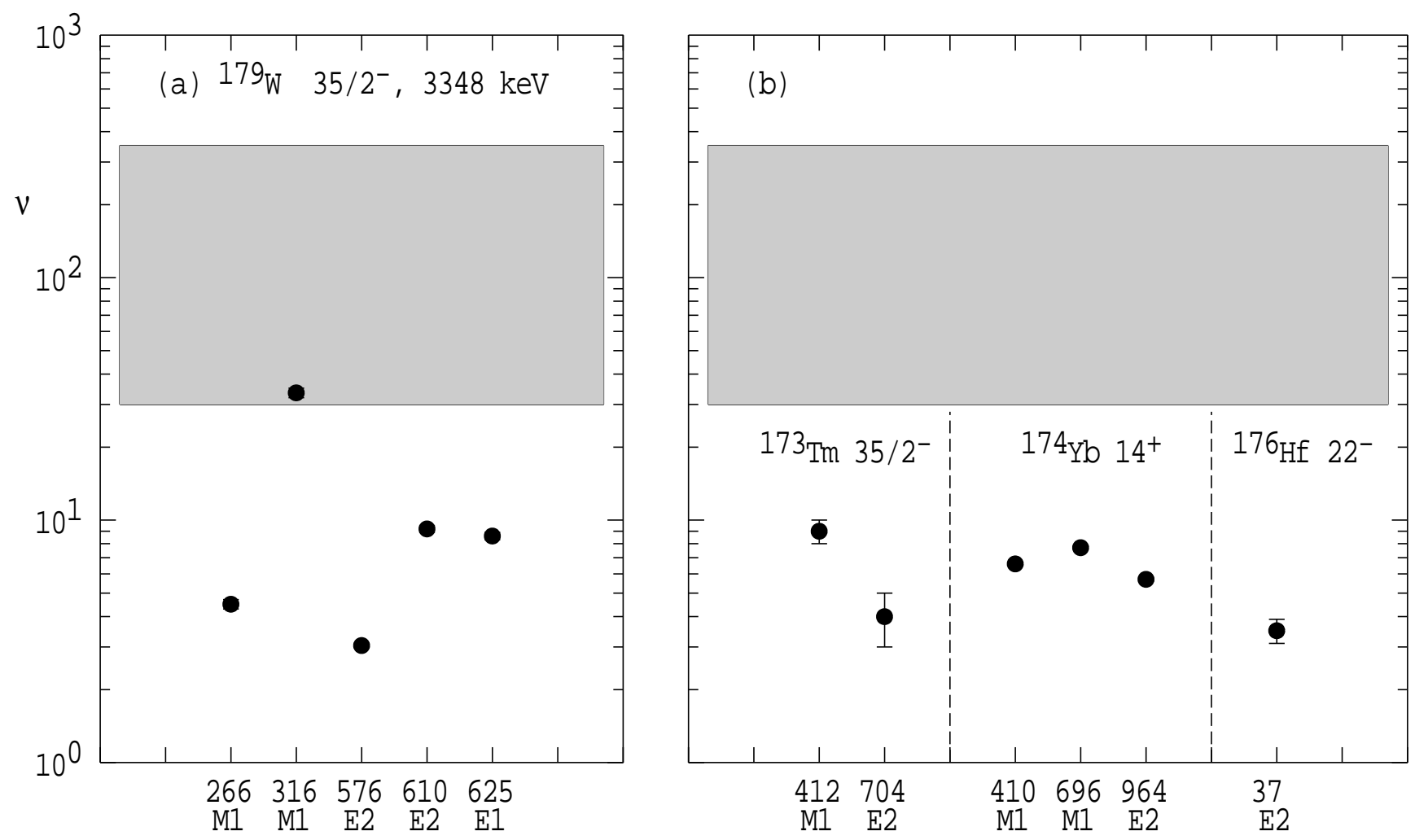

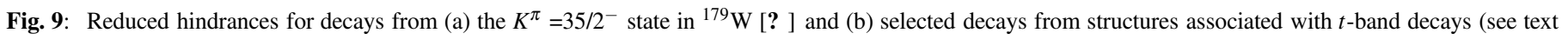
for details).

and another from an interfering band based on a $K^{\pi}=23 / 2^{-}$bandhead. The main branch at $610 \mathrm{keV}$ was, in fact, a decay to the interfering band.

Furthermore, the unusual properties of the interfering band were shown to arise from a process that was predicted to occur near mid-shell with the coupling of the $7 / 2^{+}[633]$ and $9 / 2^{+}[624]\left(i_{13 / 2}\right)$ orbitals. This leads to a band with $K \sim 8$ and significant aligned angular momentum, a phenomenon that is balanced midway between the coupling limits of rotation alignment and deformation alignment, and was originally termed "Fermi alignment" by Frauendorf [? ]. Such a band had been identified in the even-even neighbor, ${ }^{180} \mathrm{~W}$ [? ]. Its wavefunction contains a narrow, but finite, admixture of $K$ values, centered on $\mathrm{K}=8$. Coupling of an additional (spectator) nucleon, such as the $7 / 2^{-}[514]$ neutron in ${ }^{179} \mathrm{~W}$, leads to a "t-band" with $K \sim 23 / 2$ in that nucleus [? ].

Thus, this specific case involves both two- and three-state mixing, similar to that discussed above (Sect.4.2), and $K$ admixtures induced by the rotational motion in the final states lead to anomalously-low reduced hindrances. These hindrances for ${ }^{179} \mathrm{~W}$ are collected in Fig. 9(a). (Related effects were reported for ${ }^{174} \mathrm{Hf}$ and ${ }^{175} \mathrm{Hf}[?$ ].) There are also a number of transitions associated with $t$-band structures in other nuclei exhibiting anomalously low hindrances. They have yet to be explained quantitatively and do not seem to be caused by random mixing with specific states, or other factors (see below). They all correspond to transitions between multi-quasiparticle states which differ only by an additional $\mathrm{K}^{\pi}=8^{+}$component (the $t$-band structure) in the configuration. Well-defined examples include the decays between the four-quasiparticle $K^{\pi}=14^{+}$isomer in ${ }^{174} \mathrm{Yb}$ and members of the band based on the two-particle $K^{\pi}=6^{+}$configuration [? ]; the five-quasiparticle $K^{\pi}=35 / 2^{-}$isomer in ${ }^{173} \mathrm{Tm}$ and its decays to members of the three-quasiparticle $K^{\pi}=19 / 2^{-}$band [? ], and the low-energy $E 2$ branch from the six-quasiparticle $K^{\pi}=22^{-}$isomer in ${ }^{176} \mathrm{Hf}$ to the $20^{-}$member of the $K^{\pi}=14^{-}$band [? ]. All have similar, low, reduced hindrances, as illustrated in Fig. 9 b). In principle, 
these should be amenable to detailed modeling, but this has yet to be demonstrated.

Effects such as specific state mixing will be superimposed on more general phenomena, such as an indirect dependence on deformation or configuration, as will be discussed.

\subsection{Mixing in the ground-state bands: correlation with $N_{p} N_{n}$ and $\mathscr{J}^{(2)} / \mathscr{J}^{(1)}$}

As intimated earlier, the decay of isomers in even-even nuclei into the states of the ground-state collective sequences will also be controlled by mixing in the final states, often the $J^{\pi}=8^{+}$ground-state band members in particular. Coriolis antipairing, for example, implies non-zero $K$ values for these states, as does the phenomenon of rotation alignment or backbending. These phenomena depend on the position of the Fermi level relative to the high- $j$ orbitals and also on the magnitude and stiffness of the deformation. This is the rationale for a possible correlation between hindrances and moments of inertia and/or the number of valence particles.

The first of these considerations led to the evaluation of reduced hindrances for the $\Delta K=8, E 1$ decays of the $8^{-}$isomers in the $\mathrm{Hf}$ isotopes (see Sect. 4.1) as a function of the ratio of dynamic and static moments of inertia, $\mathscr{J}^{(2)} / \mathscr{J}^{(1)}$, of states in the ground-state band [?]. This parameter provides a qualitative guide to the amount of mixing due to rotation. A clear fall of $f_{v}$ with

increasing $\mathscr{J}^{(2)} / \mathscr{J}^{(1)}$ ratios above unity was evident and has been confirmed in subsequent studies. (See Sect. 4.8 below for a global evaluation.)

A more specific issue arises when the final states are close to, or in, the frequency range where alignment occurs, resulting in a specific change in character and mixed $K$ values from the $s$-band states. This was evaluated, for example, in the study by Bruce $e t$ al. [?] of the $8^{-}$isomers in the $N=74$ isotones, in which a $h_{9 / 2}^{2}$ proton alignment occurs in the ground-state band. The study used a band-mixing model combined with an expansion in terms of rotational $d$ functions to quantify the $K$ admixtures. It was successful in accounting for the fall in $f_{v}$ of the $E 1$ decays in the $8^{-}$sequence from ${ }^{130} \mathrm{Ba}$ to ${ }^{138} \mathrm{Gd}$.

The analysis in terms of the $N_{p} N_{n}$ product, using the number of valence protons $\left(\mathrm{N}_{p}\right)$ and neutrons $\left(\mathrm{N}_{n}\right)$ as a method of cataloging deformation effects (see Ref. [? ]) is a more global approach (of which a $\mathscr{J}^{(2)} / \mathscr{J}^{(1)}$ correlation could be viewed as a subset) with a more specific parameter. To the extent that the single parameter $N_{p} N_{n}$ reflects the characteristics of the deformation, one would expect a correlation with the hindrances since i) lower deformation implies higher rotational frequencies (for a given spin) and larger Coriolis effects, hence more $K$ mixing in both initial and final states, and ii) low values of $N_{p} N_{n}$ will encompass transitional nuclei where $\gamma$-softness effects could lead to loss in the identity of $K$, essentially through mixing and, hence, low hindrances. Various studies examining selected characteristics of the $A \sim 130,180$ and 250 regions have been reported by Walker and collaborators [? ? ? ? ? ]. A relatively robust correlation is observed when specific groups of deexcitation of long-lived states are isolated, such as the decay of two-quasiparticle isomers in even-even isotopes, but not surprisingly, the correlation, initially evident in Ref. [? ], becomes less obvious once more cases are included, such as those involving different seniority isomers (three-, four-, five-quasiparticle etc.) with decays of differing forbiddenness and different configuration changes (see, for example Refs. [? ? ]). The explanation for this is that the dependence on the $N_{p} N_{n}$ product is mainly probing a final state dependence - specifically mixing in the ground-state bands, not the properties of the initial multi-quasiparticle states. One more factor which will distort the general trends is the effect of mixing (in the initial state only, in this case) with a multitude of states in regions of high level density.

\subsection{Random mixing at high level densities; correlation with excitation energy}

As discussed in Section 4.2, a small random interaction between an isomer and a close-lying collective state can result in very low hindrances. In cases where an isomer occurs significantly above the yrast line, the chance of near-degeneracies with states of 

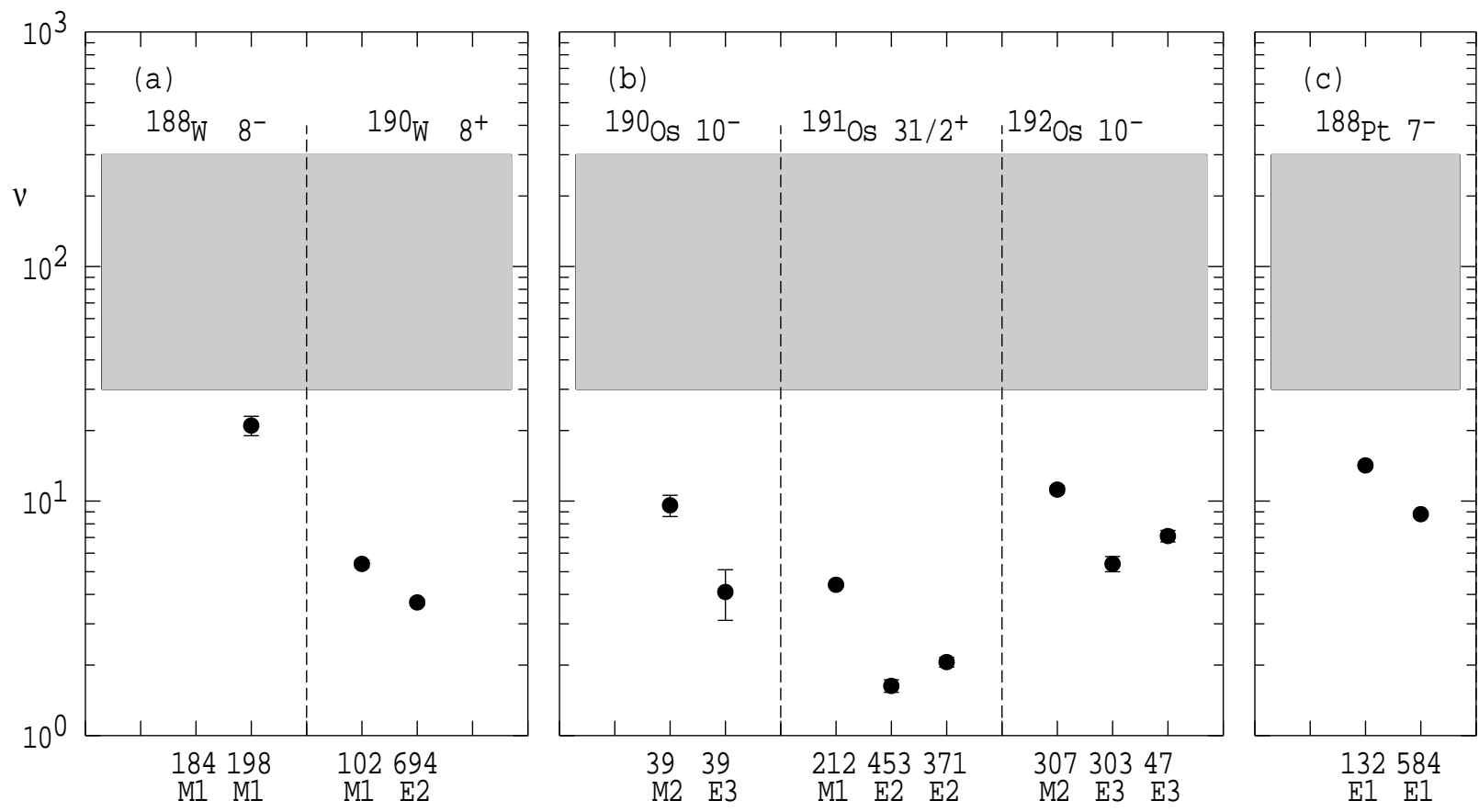

Fig. 10: Reduced hindrances for $K$-forbidden decays in the transitional tungsten, osmium and platinum isotopes.

the same spin and parity and low $K$ will increase rapidly as the level density rises, resulting in a dilution of the $K$ quantum number. This effect was studied by Walker $e t a l$. [? ] by examining the reduced hindrances of $K$-forbidden $E 2$ and $E 3$ transitions from fourand five-quasiparticle states for a range isotopes, as a function of the energy above a nominal yrast line represented by a rigid rotor, $E-E_{R}$. A clear fall of $f_{v}$ with $E-E_{R}$ was established and reproduced by a statistical model incorporating a parametrization of the density of states and a phenomenological normalization of the mixing strength.

A refinement of the approach was proposed in Ref. [? ], where only $85 \%$ of the rigid rotor value was used in estimating the relative excitation energy and more examples were incorporated [? ? ] with essentially the same result. As will be the case for any such global evaluation, even with a restriction to specific seniorities, large variations are observed, many of which can be understood through the specific processes already discussed above.

Of more general interest is the elucidation of the transition from an ordered to a chaotic quantum system in hot rotating nuclei that can, in principle, be probed through the study of isomers (e.g. Refs. [? ? ? ? ]). The hope would be to identify intrinsic states that are nominally of high- $K$ value and which would be isomeric if it were not for $K$ mixing. This becomes an experimental challenge, since such states obviously become more difficult to identify if they are very short lived. In this context, recent predictions of the presence of very long-lived, high- $K$ isomers in superdeformed nuclei [? ] offer an interesting possibility in the sense that the lifetimes are predicted to be so long that, even with significant mixing, there may be sufficient residual $K$ hindrance that the intrinsic states remain isomeric, perhaps in the nanosecond region. They would then constitute a highly sensitive probe of the proposed mixing effects.

\subsection{Gamma softness and tunneling}

There is another set of very low hindrances listed in Table 1 including what otherwise should be highly-forbidden decays directly from very high- $K$ isomers into the (nominally) $K=0$ ground-state bands. These include cases in nuclei which are well- 
deformed, such as ${ }^{174} \mathrm{Hf}$ and ${ }^{175} \mathrm{Hf}\left[?\right.$ ? ] and ${ }^{176} \mathrm{~W}$ [? ? ], as well as nuclei closer to the deformed perimeter, such as the ${ }^{182}$ Os

and ${ }^{184}$ Os isotopes [?]. The $K^{\pi}=25^{+}, 7050-\mathrm{keV}$ isomer in ${ }^{182} \mathrm{Os}$, for example, displays a series of $K$-conserving decays, but also a direct branch with $f_{v} \sim 2$ to the $24^{+}$member of the ground-state band.

Most of these cases have been interpreted in a related, qualitative fashion in terms of triaxial shape fluctuations and/or tunneling, resulting in a possible path from high- $K$ to low- $K$ states, either by changing the shape with a fixed rotating axis or by changing the orientation axis with a fixed shape (see Refs. [? ? ]). Experimentally, an important development has been the identification of structures above the $K^{\pi}=25^{+}$isomer in ${ }^{182} \mathrm{Os}$, which show irregular patterns, inconsistent with high- $K$ states in well-deformed nuclei, and were interpreted instead as evidence for multiphonon vibrations [?].

Fluctuations in the triaxial direction mean that the projection is not well defined and by implication, that $K$ is not a good quantum number. While this is well known, unlike the case of rotational effects in well-deformed nuclei, the elucidation of $K$ distributions when fluctuations (direction and shape) are present is not well developed quantitatively. The qualitative expectation that $K$ hindrances will be diluted in nuclei that are susceptible to gamma vibrations is somewhat borne out by experiment, with examples of low $f_{v}$ values emerging in heavier $\mathrm{W}$ and Os isotopes, such as ${ }^{188,190} \mathrm{~W}$ and ${ }^{190,191,192}$ Os, as depicted in Fig. 10 , Note that there are other cases discussed in the literature, where the perceived absence of isomers is taken as evidence for triaxial effects, but this is less easy to justify or quantify.

Although not directly related, it is worth noting that extensive Coulomb excitation studies of ${ }^{178}$ Hf directed originally at understanding the unexpected population of the $K^{\pi}=16^{+}$isomer from the ground-state have shown that both the mixing in many of the low- $K$ bands, and the population of states, such as the $16^{+}$isomer, must occur through complex paths [? ? ].

\subsection{Configuration changes and configuration dependence}

In addition to $K$ forbiddenness, factors which produce retardation also include the selection rules involved in significant changes of configuration. Gallagher [? ] showed that transitions between two intrinsic states involving the change of two particle orbits will be forbidden (in effect, one particle has to be a spectator) and this could occur in addition to the hindrance induced by $K$ forbiddenness. Andreeff et al. [?] relate the anomalously large $E 1$ hindrances in the decay of the $3^{+}$isomer in ${ }^{170}$ Tm to what they term " $\Omega$ forbiddenness", although Löbner questioned whether this was an effect distinct from those already covered [? ].

From the study of the decay of multi-particle isomers in spherical nuclei it is also known that large hindrances occur if several orbits are different in the initial and final states. Two representative examples are the $433-\mathrm{keV}, 23^{+} \rightarrow 21^{+}$, and $747-\mathrm{keV}, 22^{+} \rightarrow 20^{+}$ $E 2$ decays from the corresponding isomers in ${ }^{210} \mathrm{Rn}$ and ${ }^{212} \mathrm{Rn}$, which involve the change of several neutron orbitals in each case (core-excited to non core-excited states) and have hindrances of $3 \times 10^{3}$ and $3 \times 10^{4}$, respectively [? ? ]. Within the decays of multiquasiparticle states in deformed nuclei, the fact that there are usually common component configurations in the initial and final states, a consequence of the building-block scenario discussed in Section 3.1, should be reflected, at some level, in the transition strengths; particularly so when a one-particle transition is involved with a common spectator group, when these transitions might or might not be $K$ forbidden. The obvious cases to examine initially are those where the change involves either a $\pi^{2} \rightarrow 0$ or $v^{2} \rightarrow 0$ transition (in simplified nomenclature). An interesting case in this context would be the decay of an $8^{+}$intrinsic state with the two-neutron configuration implicated in the $t$-band structures (Section 4.3), but such a strength is at present not known.

One example was pointed out by Kondev et al. [? ] from the study of multi-quasiparticle isomers in ${ }^{176} \mathrm{Ta}$ and ${ }^{178} \mathrm{Ta}$. The E2 decays from the $6^{+}$isomers in ${ }^{174} \mathrm{Hf},{ }^{176} \mathrm{Hf}$ and ${ }^{178} \mathrm{Hf}$, in which the dominant configurations are $\pi^{2}$, mixed $\left(\pi^{2}+v^{2}\right)$, and $\pi^{2}$, have reduced hindrances of 19,43 and 17 , respectively. These match the values of $f_{v}=15$ for the $K^{\pi}=14^{-} \rightarrow 8^{-}$transition in ${ }^{176}$ Ta, $f_{v}$ 


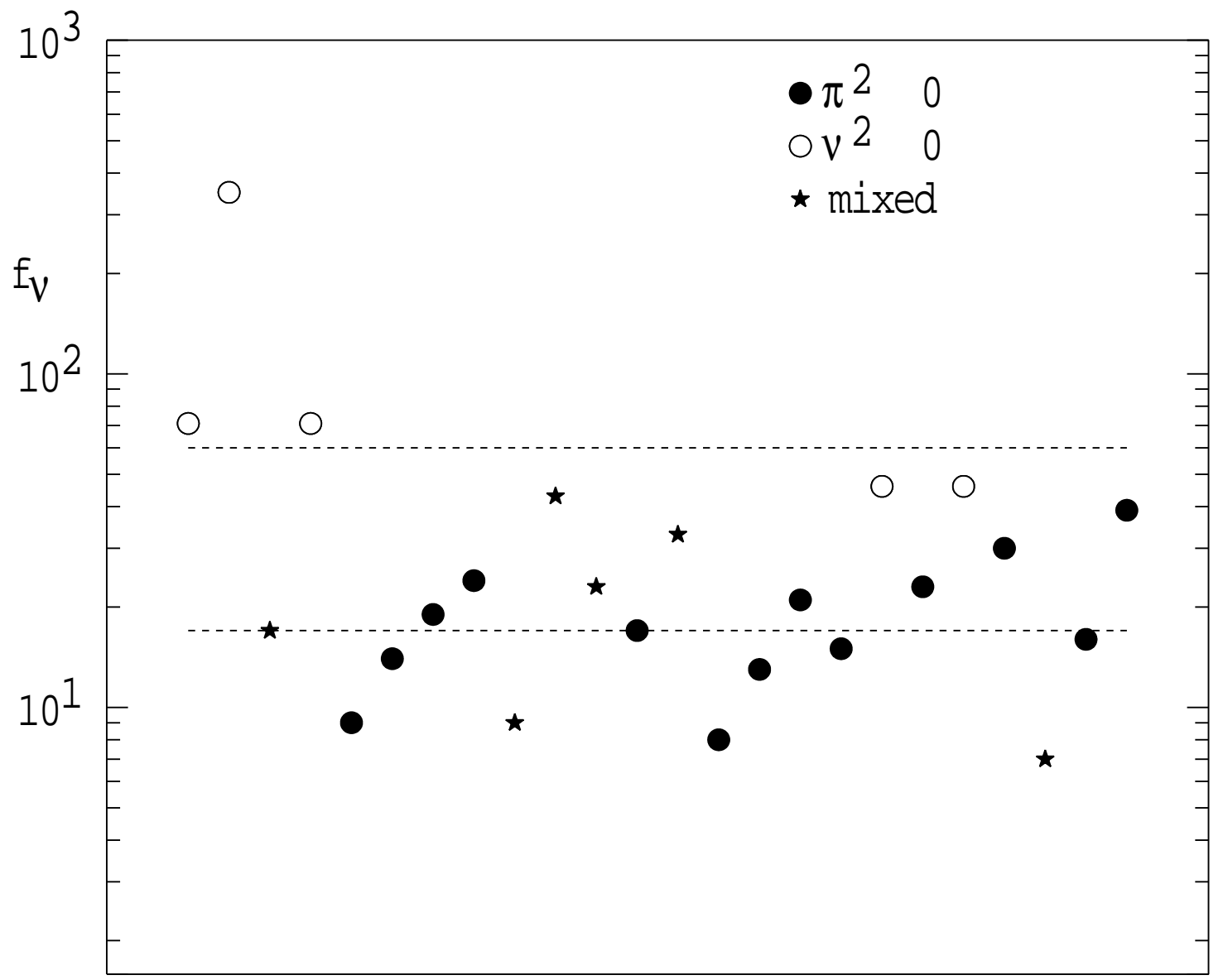

Fig. 11: Reduced hindrances for $\Delta \mathrm{K}=6, E 2$ transitions with related proton and neutron configuration changes as discussed in the text and listed in Table $\mathrm{A}$ (The points are plotted in the same order as listed in the table.)

$=46$ for the $K^{\pi}=14^{-} \rightarrow 8^{-}$transition in ${ }^{178} \mathrm{Ta}$, and $f_{v}=16$ for the $K^{\pi}=15^{-} \rightarrow 9^{-}$transition in ${ }^{180} \mathrm{Ta}$. The different $K$ values in the last case simply reflect the change in the spectator neutron.

A more comprehensive list of related transitions is given Table $\mathrm{A}$ and illustrated in Fig. 11 In general, it seems that all $v^{2} \rightarrow 0$ decays are slower (larger $f_{V}$ ) than the corresponding $\pi^{2} \rightarrow 0$ transitions, although there are fewer cases of the former, and the latter also show large variations. A number of these transitions involve mixed configurations, as indicated in both the figure and the table, either from known configurations or from random mixing. For example, the decay of the $K^{\pi}=13^{+}$isomer in ${ }^{174}$ Lu is less hindered than one may expect for a $v^{2} \rightarrow 0$ transition. However, this is a well-documented case of an accidental degeneracy, resulting in a fast transition, as discussed in Sect. 4.2 The same applies for the $K^{\pi}=21 / 2^{-}$case in ${ }^{179} \mathrm{Ta}[?]$ and the ${ }^{175} \mathrm{Hf}, K^{\pi}=35 / 2^{-}$decay [? ].

Different configurations and, thus, configuration changes also occur for the $K^{\pi}=8^{-}$isomers. Two-neutron configurations are assigned for the isomers at $N=74\left(v 7 / 2^{+}[404], 9 / 2^{-}[514]\right), N=106\left(v 7 / 2^{-}[514], 9 / 2^{+}[624]\right)$ and $N=150\left(v 7 / 2^{+}[624]\right.$, $9 / 2^{-}$[734]), while those at $Z=72$ are two-proton cases $\left(\pi 7 / 2^{+}[404], 9 / 2^{-}[514]\right)$, as is the $8^{-}$isomer in ${ }^{254} \mathrm{No}\left(\pi 7 / 2^{-}[514]\right.$, $\left.9 / 2^{+}[624]\right)$. While there is no clear configuration dependence of the strengths of corresponding $E 1$ transitions between the $K^{\pi}=8^{-}$ isomer and the $8^{+}$member of the $\mathrm{K}^{\pi}=0^{+}$, ground-state band, $\left[8^{-}\right] \rightarrow 0^{+}$, comparable to that observed for the $E 2$ transitions, there is some clustering of the $\mathrm{f}_{v}$ values around $\mathrm{f}_{v} \sim 20-40$ in the $A=130$ region, those in the mass-180 region have $\mathrm{f}_{v} \sim 70-90$ and much 
Table A

Configurations changes for selected E2 transitions in the mass-180 region.

\begin{tabular}{|c|c|c|c|c|c|}
\hline & $\left.\mathrm{K}^{\pi}, \mathrm{J}^{\pi} a\right)$ & Configuration $\left.^{b}\right)$ & Transition & $\mathrm{f}_{v}^{c)}$ & Comment \\
\hline \multirow[t]{2}{*}{${ }^{174} \mathrm{Tm}$} & i: $14^{-}, 14^{-}$ & $v 5 / 2^{-}, 7 / 2^{-}, 9 / 2^{+} \otimes \pi 7 / 2^{-}$ & & & \\
\hline & $\mathrm{f}: 8^{-}, 12^{-}$ & $v 9 / 2^{+} \otimes \pi 7 / 2^{-}$ & $v^{2}\left[6^{+}\right] \rightarrow\left[0^{+}\right]$ & 71 & \\
\hline \multirow[t]{2}{*}{${ }^{174} \mathrm{Yb}$} & i: $6^{+}, 6^{+}$ & $v 5 / 2^{-}, 7 / 2^{-}$ & & & \\
\hline & $\mathrm{f}: 0^{+}, 4^{+}$ & & $v^{2}\left[6^{+}\right] \rightarrow\left[0^{+}\right]$ & 350 & \\
\hline \multirow[t]{2}{*}{${ }^{174} \mathrm{Lu}$} & $\mathrm{i}: 13^{+}, 13^{+}$ & $v 5 / 2^{-}, 7 / 2^{-}, 7 / 2^{+} \otimes \pi 7 / 2^{+}$ & & & \\
\hline & $\mathrm{f}: 7^{+}, 11^{+}$ & $v 7 / 2^{+} \otimes \pi 7 / 2^{+}$ & $v^{2}\left[6^{+}\right] \rightarrow\left[0^{+}\right]$ & 17 & accidental mixing \\
\hline \multirow[t]{2}{*}{${ }^{175} \mathrm{Lu}$} & i: $19 / 2^{+}, 19 / 2^{+}$ & $v 5 / 2^{-}, 7 / 2^{-} \otimes \pi 7 / 2^{+}$ & & & \\
\hline & $\mathrm{f}: 7 / 2^{+}, 15 / 2^{+}$ & $\pi 7 / 2^{+}$ & $v^{2}\left[6^{+}\right] \rightarrow\left[0^{+}\right]$ & 71 & \\
\hline \multirow[t]{2}{*}{${ }^{172} \mathrm{Hf}$} & $\mathrm{i}: 6^{+}, 6^{+}$ & $\pi 5 / 2^{+}, 7 / 2^{+}$ & & & \\
\hline & $\mathrm{f}: 0^{+}, 4^{+}$ & & $\pi^{2}\left[6^{+}\right] \rightarrow\left[0^{+}\right]$ & 9 & \\
\hline \multirow[t]{2}{*}{$173 \mathrm{Hf}$} & i: $19 / 2^{+}, 19 / 2^{+}$ & $v 7 / 2^{+} \otimes \pi 5 / 2^{+}, 7 / 2^{+}$ & & & \\
\hline & $\mathrm{f}: 7 / 2^{+}, 15 / 2^{+}$ & $v 7 / 2^{+}$ & $\pi^{2}\left[6^{+}\right] \rightarrow\left[0^{+}\right]$ & $\leq 14$ & \\
\hline \multirow[t]{2}{*}{${ }^{174} \mathrm{Hf}$} & i: $6^{+}, 6^{+}$ & $\pi 5 / 2^{+}, 7 / 2^{+}$ & & & \\
\hline & $\mathrm{f}: 0^{+}, 4^{+}$ & & $\pi^{2}\left[6^{+}\right] \rightarrow\left[0^{+}\right]$ & 19 & \\
\hline \multirow[t]{2}{*}{${ }^{175} \mathrm{Hf}$} & i: $19 / 2^{+}, 19 / 2^{+}$ & $v 7 / 2^{+} \otimes \pi 5 / 2^{+}, 7 / 2^{+}$ & & & \\
\hline & $\mathrm{f}: 7 / 2^{+}, 15 / 2^{+}$ & $v 7 / 2^{+}$ & $\pi^{2}\left[6^{+}\right] \rightarrow\left[0^{+}\right]$ & 24 & \\
\hline \multirow[t]{2}{*}{${ }^{175} \mathrm{Hf}$} & i: $35 / 2^{-}, 35 / 2^{-}$ & $v 5 / 2^{-}, 7 / 2^{-}, 7 / 2^{+} \otimes \pi 7 / 2^{+}, 9 / 2^{-}$ & & & \\
\hline & f: $23 / 2^{-}, 31 / 2^{-}$ & $v 7 / 2^{+} \otimes \pi 7 / 2^{+}, 9 / 2^{-}$ & $v^{2}\left[6^{+}\right] \rightarrow\left[0^{+}\right]$ & 9 & accidental mixing \\
\hline \multirow[t]{2}{*}{${ }^{176} \mathrm{Hf}$} & $\mathrm{i}: 6^{+}, 6^{+}$ & $61 \% v 5 / 2^{-}, 7 / 2^{-}+39 \% \pi 5 / 2^{+}, 7 / 2^{+}$ & & & \\
\hline & $\mathrm{f}: 0^{+}, 4^{+}$ & & $v^{2}\left[6^{+}\right] \rightarrow\left[0^{+}\right]$ & 43 & configuration mixing \\
\hline \multirow[t]{2}{*}{${ }^{176} \mathrm{Hf}$} & i: $14^{-}, 14^{-}$ & $v 5 / 2^{-}, 7 / 2^{-} \otimes \pi 7 / 2^{+}, 9 / 2^{-}$ & & & \\
\hline & $\mathrm{f}: 8^{-}, 12^{-}$ & $38 \% v 7 / 2^{-}, 9 / 2^{+}+62 \% \pi 7 / 2^{+}, 9 / 2^{-}$ & $v^{2}\left[6^{+}\right] \rightarrow\left[0^{+}\right]$ & 23 & configuration mixing \\
\hline \multirow[t]{2}{*}{${ }^{178} \mathrm{Hf}$} & $\mathrm{i}: 6^{+}, 6^{+}$ & $\pi 5 / 2^{+}, 7 / 2^{+}$ & & & \\
\hline & $\mathrm{f}: 0^{+}, 4^{+}$ & & $\pi^{2}\left[6^{+}\right] \rightarrow\left[0^{+}\right]$ & 17 & \\
\hline \multirow[t]{2}{*}{${ }^{178} \mathrm{Hf}$} & i: $14^{-}, 14^{-}$ & $v 7 / 2^{-}, 9 / 2^{+} \otimes \pi 5 / 2^{+}, 7 / 2^{+}$ & & & \\
\hline & $\mathrm{f}: 8^{-}, 12^{-}$ & $64 \% v 7 / 2^{-}, 9 / 2^{+}+36 \% \pi 9 / 2^{-}, 7 / 2^{+}$ & $\pi^{2}\left[6^{+}\right] \rightarrow\left[0^{+}\right]$ & 33 & configuration mixing \\
\hline \multirow[t]{2}{*}{${ }^{180} \mathrm{Hf}$} & $\mathrm{i}: 6^{+}, 6^{+}$ & $\pi 5 / 2^{+}, 7 / 2^{+}$ & & & \\
\hline & $\mathrm{f}: 0^{+}, 4^{+}$ & & $\pi^{2}\left[6^{+}\right] \rightarrow\left[0^{+}\right]$ & $\leq 8$ & \\
\hline \multirow[t]{2}{*}{${ }^{173} \mathrm{Ta}$} & i: $21 / 2^{-}, 21 / 2^{-}$ & $\pi 5 / 2^{+}, 7 / 2^{+}, 9 / 2^{-}$ & & & \\
\hline & f: $9 / 2^{-}, 17 / 2^{-}$ & $\pi 9 / 2^{-}$ & $\pi^{2}\left[6^{+}\right] \rightarrow\left[0^{+}\right]$ & 13 & \\
\hline \multirow[t]{2}{*}{${ }^{175} \mathrm{Ta}$} & i: $21 / 2^{-}, 21 / 2^{-}$ & $\pi 5 / 2^{+}, 7 / 2^{+}, 9 / 2^{-}$ & & & \\
\hline & $\mathrm{f}: 9 / 2^{-}, 17 / 2^{-}$ & $\pi 9 / 2^{-}$ & $\pi^{2}\left[6^{+}\right] \rightarrow\left[0^{+}\right]$ & 21 & \\
\hline \multirow[t]{2}{*}{${ }^{176} \mathrm{Ta}$} & i: $14^{-}, 14^{-}$ & $v 7 / 2^{+} \otimes \pi 5 / 2^{+}, 7 / 2^{+}, 9 / 2^{-}$ & & & \\
\hline & $\mathrm{f}: 8^{-}, 12^{-}$ & $v 7 / 2^{+} \otimes \pi 9 / 2^{-}$ & $\pi^{2}\left[6^{+}\right] \rightarrow\left[0^{+}\right]$ & 15 & \\
\hline \multirow[t]{2}{*}{${ }^{176} \mathrm{Ta}$} & i: $20^{-}, 20^{-}$ & $v 5 / 2^{-}, 7 / 2^{-}, 7 / 2^{+} \otimes \pi 5 / 2^{+}, 7 / 2^{+}, 9 / 2^{-}$ & & & \\
\hline & $\mathrm{f}: 14^{-}, 18^{-}$ & $v 7 / 2^{+} \otimes \pi 5 / 2^{+}, 7 / 2^{+}, 9 / 2^{-}$ & $v^{2}\left[6^{+}\right] \rightarrow\left[0^{+}\right]$ & 46 & \\
\hline \multirow[t]{2}{*}{${ }^{177} \mathrm{Ta}$} & i: $21 / 2^{-}, 21 / 2^{-}$ & $\pi 5 / 2^{+}, 7 / 2^{+}, 9 / 2^{-}$ & & & \\
\hline & $\mathrm{f}: 9 / 2^{-}, 17 / 2^{-}$ & $\pi 9 / 2^{-}$ & $\pi^{2}\left[6^{+}\right] \rightarrow\left[0^{+}\right]$ & 23 & \\
\hline \multirow[t]{2}{*}{${ }^{178} \mathrm{Ta}$} & i: $15^{-}, 15^{-}$ & $v 5 / 2^{-}, 7 / 2^{-}, 9 / 2^{+} \otimes \pi 9 / 2^{-}$ & & & \\
\hline & $\mathrm{f}: 9^{-}, 13^{-}$ & $v 9 / 2^{+} \otimes \pi 9 / 2^{-}$ & $v^{2}\left[6^{+}\right] \rightarrow\left[0^{+}\right]$ & 46 & \\
\hline \multirow[t]{2}{*}{${ }^{178} \mathrm{Ta}$} & i: $21^{-}, 21^{-}$ & $v 5 / 2^{-}, 7 / 2^{-}, 9 / 2^{+} \otimes \pi 5 / 2^{+}, 7 / 2^{+}, 9 / 2^{-}$ & & & \\
\hline & $\mathrm{f}: 15^{-}, 19^{-}$ & $v 5 / 2^{-}, 7 / 2^{-}, 9 / 2^{+} \otimes \pi 9 / 2^{-}$ & $\pi^{2}\left[6^{+}\right] \rightarrow\left[0^{+}\right]$ & 30 & \\
\hline \multirow[t]{2}{*}{${ }^{179} \mathrm{Ta}$} & i: $21 / 2^{-}, 21 / 2^{-}$ & $\pi 5 / 2^{+}, 7 / 2^{+}, 9 / 2^{-}$ & & & \\
\hline & $\mathrm{f}: 9 / 2^{-}, 17 / 2^{-}$ & $\pi 9 / 2^{-}$ & $\pi^{2}\left[6^{+}\right] \rightarrow\left[0^{+}\right]$ & 7 & accidental mixing \\
\hline${ }^{180} \mathrm{Ta}$ & i: $15^{-}, 15^{-}$ & $v 9 / 2^{+} \otimes \pi 5 / 2^{+}, 7 / 2^{+}, 9 / 2^{-}$ & & & \\
\hline & $\mathrm{f}: 9^{-}, 13^{-}$ & $v 9 / 2^{+} \otimes \pi 9 / 2^{-}$ & $\pi^{2}\left[6^{+}\right] \rightarrow\left[0^{+}\right]$ & 16 & \\
\hline${ }^{181} \mathrm{Ta}$ & i: $21 / 2^{-}, 21 / 2^{-}$ & $\pi 5 / 2^{+}, 7 / 2^{+}, 9 / 2^{-}$ & & & \\
\hline & f: $9 / 2^{-}, 17 / 2^{-}$ & $\pi 9 / 2^{-}$ & $\pi^{2}\left[6^{+}\right] \rightarrow\left[0^{+}\right]$ & 39 & \\
\hline
\end{tabular}

a) i: initial state; f: final state

$\left.{ }^{b}\right)(v) 7 / 2^{-}: 7 / 2^{-}$[514], 7/2+: 7/2+ [633], 5/2- : 5/2- [512], 9/2+ : 9/2+ [624].

( $\pi$ ) $7 / 2^{-}: 7 / 2^{-}[523], 5 / 2^{+}: 5 / 2^{+}[402], 7 / 2^{+}: 7 / 2^{+}[404], 9 / 2^{-}: 9 / 2^{-}[514]$.

$\left.{ }^{c}\right)$ Rounded off values. See Table 1 for details.

larger values of $\mathrm{f}_{v} \sim 170-200$ are seen in the heavier, mass-250 region. 
The full systematics for the $K^{\pi}=8^{-}$isomers covering the three regions of deformation is given in Fig. 12 , as a function of several parameters, as discussed in Section 4 . Several remarks are necessary before discussing these results. First, the parameter $E-E_{\text {rot }}$ in Fig. 12 (a) is meant to act as an indirect guide to level densities above the yrast line, but such densities also depend on mass number and other nuclear properties (such as pairing) so that a single global dependence should not be expected. Second, the two lower representations (Fig. 12 b) and 12 (c)) use independent variables, which are related in terms of the physics, the $N_{p} N_{n}$

product being a rather approximate surrogate for deformation, while the $\mathscr{J}^{(2)} / \mathscr{J}^{(1)}$ ratio of moments depends on $K$ mixing and/or on the change of softness in the ground-state bands.

The two main effects underlying these systematics are mixing in the initial isomeric state, which produces part of the correlated decrease with energy in Fig. 12 a) and mixing in the final state, in this case the $8^{+}$member of the ground-state band, as evidenced by Fig. 12 (b) and (c).

Note that the curves drawn on these figures are not fits, but are rather meant to guide the discussion. The two lines in Fig. 12(a), for example, are selected to follow the trajectories of the hindrances for the $8^{-}$state decays in the mass- 180 region. There is a clear difference in both magnitude and energy dependence between the set of cases depending on whether the $\left(8^{-} \rightarrow 8^{+}\right)$transition corresponds to either a $v^{2} \rightarrow 0$ or a $\pi^{2} \rightarrow 0$ configuration change, reflecting the earlier discussion on configuration dependency. The points enclosed in the higher rectangle in Fig. 12 a) are for ${ }^{180} \mathrm{Hf}$ and ${ }^{182} \mathrm{Hf}$, while the lower rectangle contains data for ${ }^{174} \mathrm{Er},{ }^{176} \mathrm{Hf}$ and ${ }^{178} \mathrm{~W}$, the $N=106$ isotones discussed earlier.

The points which are significantly below the relatively smooth trend, highlighted by the ellipse in Fig. 12 (c), correspond to the $8^{-}$state decays in ${ }^{170} \mathrm{Hf}$ and ${ }^{172} \mathrm{Hf}$. They are also low in Fig. 12 (b), because they are at relatively high excitation energies compared to the yrast line (Fig. 12(a)), and, therefore, reside in regions of higher level density. The main point here is that each of these effects (configuration dependence, random mixing etc.) will be superimposed on what might otherwise be a general trend. Future developments in the understanding of the trends will require combining different effects, which can be modeled, at least approximately.

\section{Fission branches in the Fermium region}

In principle, high- $K$ isomers should occur in both the first (normal-deformed) and second (superdeformed) wells in the heavy nuclei that exhibit multiple minima in their nuclear potentials. Following the early discovery of two-quasiparticle isomers in the first well of ${ }^{250} \mathrm{Fm}(Z=100)$ and ${ }^{254} \mathrm{No}(Z=102)[?]$, a number of spectroscopic studies have been carried out in the Fermium region using various techniques, which have differing sensitivities, both in terms of decay branches ( $\alpha$ decay, $\gamma$ decay, fission, etc.) and lifetime ranges. As listed in Table 1 there are now numerous (mainly two-quasiparticle) isomers identified in this region, but only a limited number for which spontaneous fission branches (or limits) have been measured. Those cases are summarized in Table B and will be discussed later in this section.

In contrast, the so-called Fission Isomers correspond to decays from states in the second well. These lie at the other extreme in terms of known properties, in that nothing is known of their spin, parity, or decay branches, except for the spontaneous fission half-lives. They have been extensively discussed and documented [? ? ? ? ], including six cases in the even-even Pu and Cm isotopes where two decays are seen corresponding to what are assumed to be the decay of a high- $K$, two-quasiparticle state and of the $0^{+}$"ground" state. In all of these, the half-lives of the two-quasiparticle states (at excitation energies with respect to the second-well minimum of about $\sim 0.9-1.3 \mathrm{MeV}$ ) are in the tens of nanosecond range. This is relatively short, but it is orders of 

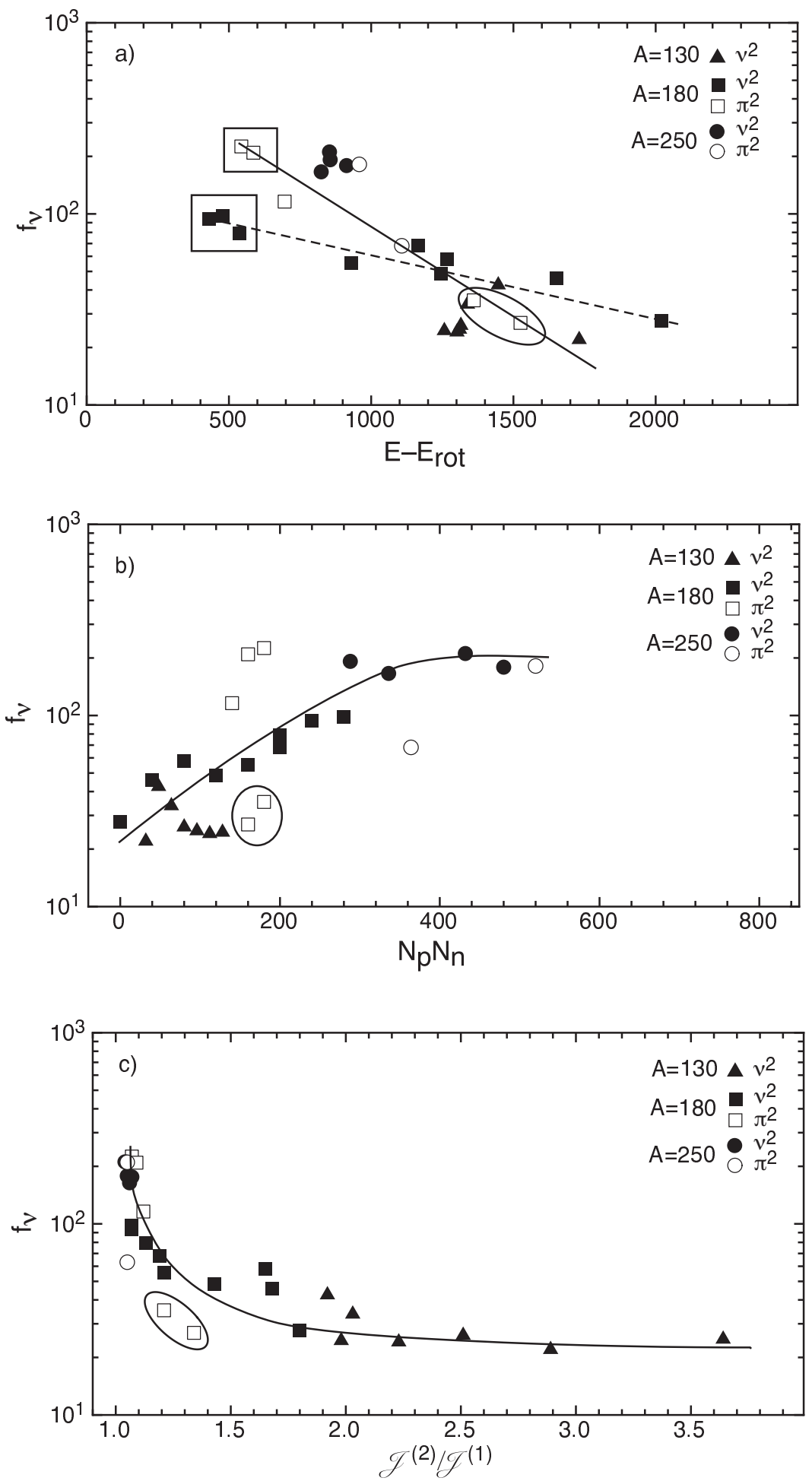

Fig. 12: Forbidden $\Delta K=8 E 1$ decays from $K^{\pi}=8^{-}$isomers in different deformed regions: (a) as a function of the excitation energy with respect to the yrast line $\left(E-E_{r o t}\right)$, where $\mathrm{E}_{r o t}=\frac{\hbar}{2 \mathfrak{S}} \cdot J \cdot(J+1)+\Delta$, with $\frac{\hbar}{2 \mathfrak{S}}=\frac{36300}{0.707 \cdot \mathrm{A}^{5 / 2} \cdot(1+\beta)}$ in $\mathrm{keV}, \beta=0.25$ and $\Delta=0, \Delta_{v}, \Delta_{\pi}$ and $\Delta_{v}+\Delta_{\pi}$ for even-N - even-Z, odd-N - even-Z, even-N odd-Z, and odd-N - odd-Z, respectively [? ]; (b) as a function of the valence particle product $N_{p} N_{n}$, and (c) as a function the ratio of dynamic $\left(\mathscr{J}(2)=4 / \Delta E_{\gamma}\right)$ and kinematic $\left(\mathscr{J}^{(1)}=(2 \cdot J-1) / E_{\gamma}\right)$ moments of inertia at $I=8$ in the ground-state band (compare also Figs. 8 and 9 of Ref. [? ]). The curves and outlined groups are discussed in the text. 
Table B

$\mathrm{K}$-isomer fission decays and fission hindrance factors.

\begin{tabular}{|c|c|c|c|c|c|c|c|c|c|}
\hline \multirow[b]{2}{*}{$\mathrm{K}^{\pi}(\hbar)$} & \multicolumn{3}{|c|}{ ground state } & \multirow[b]{2}{*}{$\mathrm{E}(\mathrm{keV})$} & \multirow[b]{2}{*}{$\mathrm{K}^{\pi}(\hbar)$} & \multicolumn{2}{|c|}{ isomer } & \multirow[b]{2}{*}{$\left.\mathrm{T}_{1 / 2}^{S F} c\right)$} & \multirow[b]{2}{*}{$\mathrm{HF}^{d)}$} \\
\hline & $\mathrm{T}_{1 / 2}^{\text {expt a) }}$ & $\mathrm{b}_{S F}(\%)^{b)}$ & $\mathrm{T}_{1 / 2}^{S F c)}$ & & & $\mathrm{T}_{1 / 2}^{\text {expt a }}$ & $\mathrm{b}_{S F}(\%)^{b)}$ & & \\
\hline \multicolumn{10}{|c|}{${ }^{244} \mathrm{Cm}(\mathrm{Z}=96, \mathrm{~N}=148)$. Data from Refs. [? ? ? ]. } \\
\hline $0^{+}$ & $18.1(1) \mathrm{yr}$ & $1.37(3) \mathrm{E}-4$ & $1.32(3) \mathrm{E}+7 \mathrm{yr}$ & 1040.2 & $6^{+}$ & $34(2) \mathrm{ms}$ & $\leq 8.0 \mathrm{E}-10$ & $\geq 127 \mathrm{yr}$ & $\geq 9.4 \mathrm{E}-6$ \\
\hline \multicolumn{10}{|c|}{${ }^{250} \mathrm{Fm}(\mathrm{Z}=100, \mathrm{~N}=150)$. Data from Refs. [? ? ? ? ? ? ]. } \\
\hline $0^{+}$ & $28.4(7) \mathrm{m}$ & $0.0069(10)$ & $286(42) \mathrm{d}$ & 1199.3 & $8^{-}$ & $1.92(5) \mathrm{s}$ & $\leq 8.2 \mathrm{E}-05$ & $\geq 26.4 \mathrm{~d}$ & $\geq 0.079$ \\
\hline \multicolumn{10}{|c|}{${ }^{256} \mathrm{Fm}(\mathrm{Z}=100, \mathrm{~N}=156)$. Data from Refs. [? ? ]. } \\
\hline $0^{+}$ & $157.6(13) \mathrm{m}$ & $91.9(3)$ & $1.029(9) \mathrm{E}+04 \mathrm{~s}$ & 1425.3 & $7^{-}$ & $70(5) \mathrm{ns}$ & $\sim 2.0 \mathrm{E}-03$ & $\sim 0.00350(25) \mathrm{s}$ & $\sim 3.40(25) \mathrm{E}-07$ \\
\hline \multicolumn{10}{|c|}{${ }^{250} \mathrm{No}(\mathrm{Z}=102, \mathrm{~N}=148)$. Data from Refs. [? ? ]. } \\
\hline $0^{+}$ & $4.9(6) \mu \mathrm{s}$ & $\sim 100 \ddagger$ & $\sim 4.9(6) \ddagger \mu \mathrm{s}$ & $800+X \neq$ & $\left(6^{+}\right)$ & $54(10) \mu \mathrm{s}$ & $\sim 100$ & $\sim 54(10) \mu \mathrm{s}$ & $\sim 11$ (2) \\
\hline \multicolumn{10}{|c|}{${ }^{254} \mathrm{No}(\mathrm{Z}=102, \mathrm{~N}=152)$. Data from Refs. [? ? ]. } \\
\hline \multirow[t]{2}{*}{$0^{+}$} & $51.2(4) \mathrm{s}$ & $0.23(1)$ & $2.22(10) \mathrm{E}+04 \mathrm{~s}$ & 1294.9 & $8^{-}$ & $265.0(14) \mathrm{ms}$ & $0.020(12)$ & $1.3(8) \mathrm{E}+03 \mathrm{~s}$ & $0.06(4)$ \\
\hline & & & & 2918.1 & $\left(16^{+}\right)$ & $183.8(16) \mu \mathrm{s}$ & $\leq 0.013$ & $\geq 1.4 \mathrm{~s}$ & $\geq 6.0 \mathrm{E}-05$ \\
\hline \multicolumn{10}{|c|}{${ }^{262} \operatorname{Rf}(\mathrm{Z}=104, \mathrm{~N}=158)$. Data from Refs. [? ? ]. } \\
\hline $0^{+}$ & $2.1(2) \mathrm{s}$ & $\sim 100$ & $2.1(2) \mathrm{s}$ & $0+X \ddagger$ & $\left(8^{-}, 9^{-}\right) \neq$ & $47(5) \mathrm{ms}$ & $\sim 100$ & $4.7(5) \mathrm{E}-02 \mathrm{~s}$ & $\sim 0.022(3)$ \\
\hline
\end{tabular}

a) Experimental half-life.

b) SF branching intensity in $\%$.

c) Partial SF half-life, $\mathrm{T}_{1 / 2}^{S F}=100 \cdot \mathrm{T}_{1 / 2}^{\text {expt }} / \mathrm{b}_{S F}$.

d) Hindrance factor, $\mathrm{HF}=\mathrm{T}_{1 / 2}^{S F}$ (isomer)/ $\mathrm{T}_{1 / 2}^{S F}$ (ground state).

magnitude longer than the values for the $0^{+}$ground states in the second well, which are characterized by half-lives in the tens of picoseconds. Examples are also known in the odd-odd ${ }^{244} \mathrm{Am}$ and ${ }^{242} \mathrm{Bk}$ isotopes [? ? ? ]. Similar to the even-even cases, they are associated with higher-seniority (presumably higher- $K$ ) states, but have much longer lifetimes.

There has been continuing activity addressing the complex question of whether such excited states would be expected to be shorter- or longer-lived, given changes in the fission barriers, $\alpha$-decay probabilities and the effects of nuclear structure (such as $K$ hindrance). Obviously, the first- and second-well states are likely to exhibit different sensitivities to these properties and to other underlying effects. However, one-dimensional barrier calculations that allow one to visualize a double-well structure are only part of the necessary considerations, since dynamics exploring a more complicated three-dimensional deformation space ultimately control fission probabilities. For example, Baran and Lojewski [? ] carried out theoretical studies predicting spontaneousfission probabilities for the high- $K$, two-quasiparticle states in ${ }^{250} \mathrm{Fm}$ and ${ }^{254}$ No using a one-dimensional approach and the $W K B$ approximation. They concluded that the rate of spontaneous fission could be orders of magnitude slower than that of the ground states, due to higher and wider fission barriers. However, their more extensive calculations [? ], which examined a range of nuclei, indicated generally that the results were very dependent on $Z$, with some examples $(Z>104)$ having faster fission and, depending on other decay branches, shorter lifetimes than the ground states.

By incorporating a dynamical treatment of pairing, Lazarev [? ] concluded that spontaneous fission of high- $K$ isomers depended critically on dynamically-induced superfluidity in the tunneling process. He also re-iterated the known sensitivity to triaxial shape distortions, which implicitly mix $K$, and pointed out the possible ambiguities that might be introduced into the experimental study of superheavy nuclei in the event that high- $K$ states were longer-lived than the corresponding ground states. Recently, the possibility that $K$ isomers could be more stable (have longer lifetimes) in superheavy elements has again been pointed out qualitatively in terms 


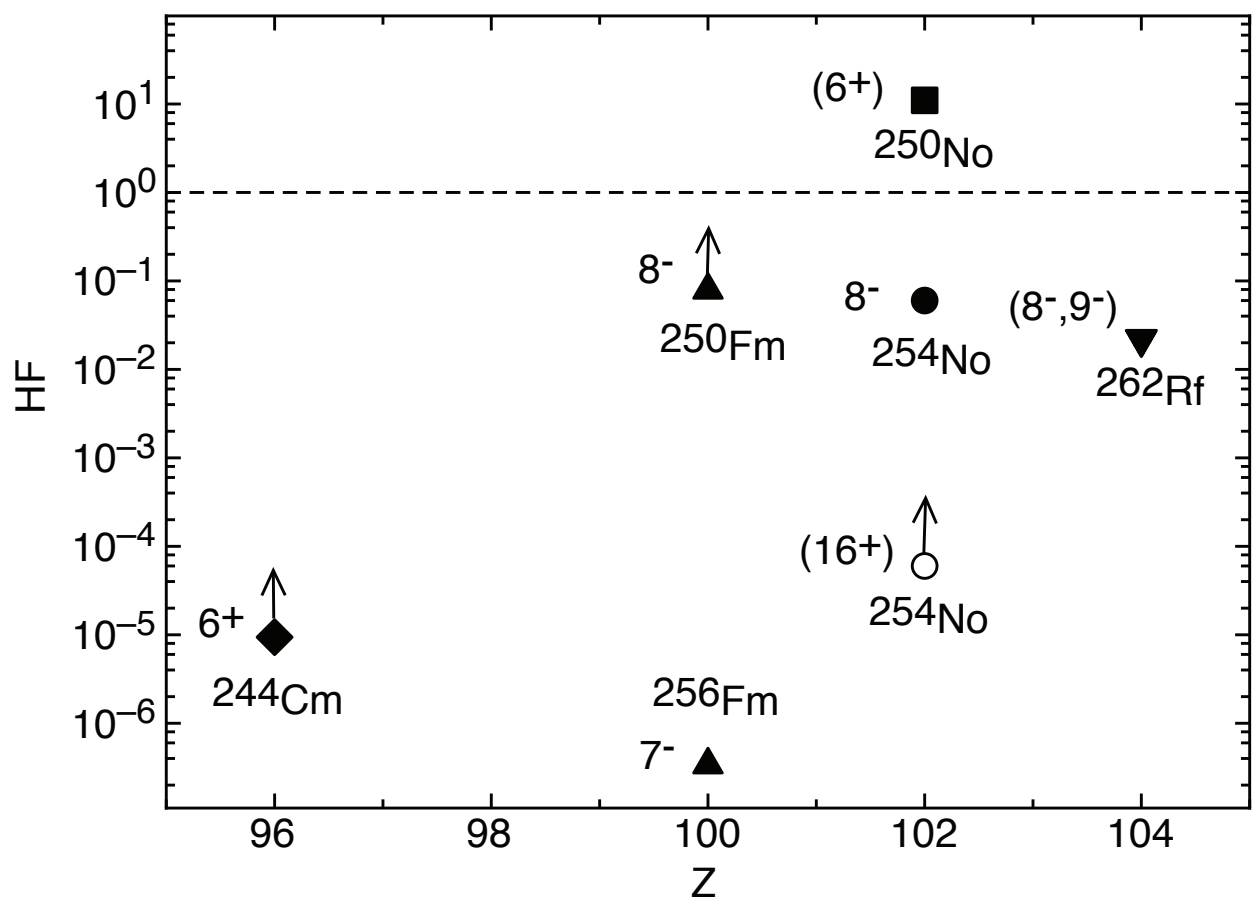

Fig. 13: Hindrances (measurements and limits) of spontaneous fission for two- and four-quasiparticle isomers relative to unity for the ground states (dashed line).

of the shape of the fission barriers when using configuration constraints in calculations of the potential energies and fission barriers, but without inclusion of dynamical effects or the calculation of lifetimes [? ? ? ].

As indicated above, experimental information on spin and parity remains limited to states in the first well. Table $\mathrm{B}$ lists those nuclei that are known to have spontaneous-fission branches. The partial fission half-lives for the ground state and the isomers are presented, together with the hindrance factor defined as $H F=T_{1 / 2}^{S F}($ isomer $) / T_{1 / 2}^{S F}($ ground state $)$. The deduced hindrance factors are also plotted in Fig. 13, In all cases, except ${ }^{250}$ No, the partial fission lifetimes for the isomers are in fact shorter than those of the respective ground state, although a number are only limits. It should also be pointed out that, in the case of ${ }^{250}$ No, the assignment of spontaneous activity to its isomer is not unambiguous. The experimental evidence at present does not indicate higher hindrances for spontaneous fission from $K$ isomers, at least not for those located in the first well.

\section{E1, M1 and E2 hindrances: dependencies}

Given the historical development of the study of hindered transitions and how they have been used to make decisions on which values are acceptable in terms of spin/parity assignments and structure effects, we show here the $E 1$ strengths in a form that partly matches the original presentation of Löbner [? ]. The important difference here is that, whereas Löbner showed a simple range for each value of $\Delta K$, with an expanded data set it is now possible to show the distribution in each case by suitable binning.

Several points emerge: the first is that, in general, the distributions are not symmetric, hence care must be taken in evaluating "normal" values. Most of the $\Delta K=8$ values, for example, fall with equal probability in a range which corresponds to $f_{v}=20-300$. Furthermore, the approximate centroid positions increase much more slowly than would be expected from the rule of thumb of 100 for each additional degree of forbiddenness [? ? ? ], indicated by the dashed line in Fig. 14

Prompted by the earlier comments regarding the use of arbitrary factors in discussing $E 1$ hindrances, and by the more complete 

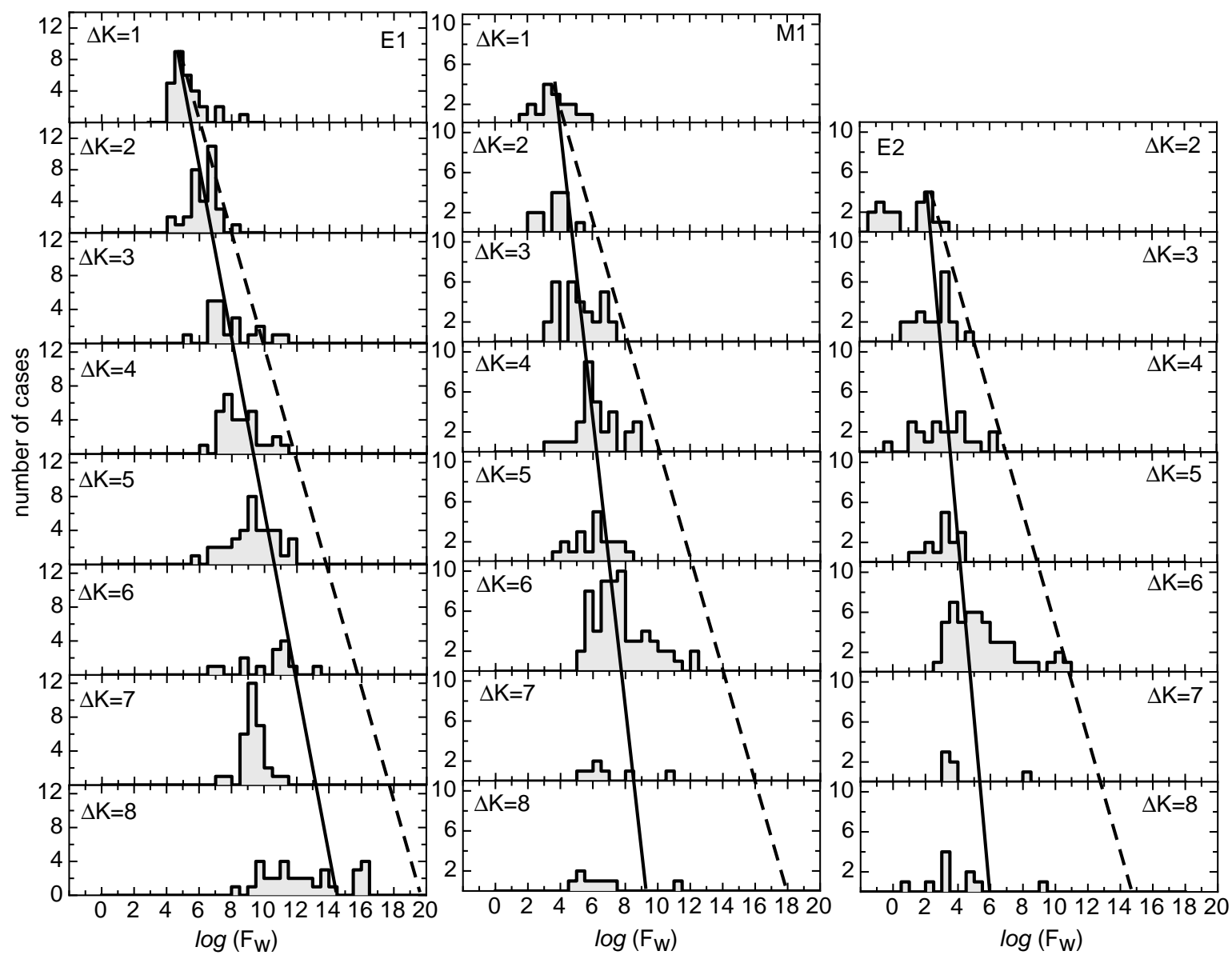

Fig. 14: Distribution of $E 1, M 1$ and $E 2$ decays as a function of $\Delta K$. The solid lines are fits to a restricted range of points, as discussed in the text. The dashed line correspond to $f_{0}=100$ (see the text for details).

systematics, the approach taken here has been to factorize the overall hindrance into a product of an underlying (intrinsic) hindrance and a factor related to the additional hindrance due to $K$ forbiddenness. That is,

$$
F=F_{0} \times f_{0}^{v}
$$

and in a logarithmic form:

$$
\log F=\log F_{0}+v \times \log f_{0} .
$$

This concurs with the inference by Löbner [? ? ] that there was a multipolarity-dependent offset in the hindrances, contrary to the expectation of the simple Rusinov rule [? ]. The centroids are plotted as a function of $\Delta K$ in Fig. 15, overlaid by several linear fits to eqn. (2), with and without the exclusion of some points (open circles).

Some comments are necessary regarding possible exceptional cases in these compilations. In particular, the $E 2$ distribution for $\Delta K=2$ (allowed) transitions has two peaks, the lower corresponding to transitions that are enhanced over the Weisskopf estimates. Examination of the latter reveals that they are probably not, and in some instance, definitely not, decays from intrinsic states. (Qualifications to this effect are included in Table1) Two long-lived states of this group, for example, are isomers in ${ }^{192}$ Os [? ] that arise from transitions within rotation-aligned structures. They are collective, enhanced, in-band transitions that result in significant state lifetimes only because of their low energies. They belong in the same category as the $2^{+}$ground-state band members in 
well-deformed, even-even nuclei, discussed in the introduction.

Obviously, the statistics in the $\Delta K=7, M 1$ and $E 2$ cases are too low to be significant in terms of the fits. The $\Delta K=8, M 1$ and $E 2$ cases have low statistics as well, although both $\Delta K=8$ distributions appear to cluster at relatively low values. Examination of these indicates that most are the $t$-band cases discussed in Sect. 4.3.

In contrast to these, the $\Delta \mathrm{K}=7, E 1$ distribution is strongly peaked, but also at a low value compared to the trend. Examination of the individual cases contributing to this peak brings out several points: essentially, all cases fit into the two categories of i) isomeric states located well above the yrast line and, therefore, influenced by K mixing, as discussed in Sect. 4.5, or ii) isomeric states with strong Coriolis $K$ mixing due to the presence of an $i_{13 / 2}$ neutron in the configuration. One example of the latter is the $\mathrm{K}^{\pi}=7^{-}$isomer in ${ }^{180} \mathrm{Os}$ (see, for example [? ], for calculation of the $K$ mixing); it decays by five $E 1$ transitions, four of which are relatively fast due to the mixing and, hence, contributing disproportionately to the apparent peak.

Notwithstanding qualifications such as these, clear correlations are evident in Fig. 15, consistent with an underlying hindrance $\left(F_{0}\right)$ for each multipolarity. Rather than including an arbitrary reduction factor that has often been used to modify experimental values for $E 1$ transitions before comparison with theory, a suggested value with a nominal error defining a range can be extracted here for $E 1, M 1$ and $E 2$ multipolarities, as listed in Table C Given sufficient statistics, this approach should be applicable to other multipolarities, as well, and this is discussed briefly for $E 3$ transitions in the next section. The analysis indicates that the extracted value of $f_{0}$ is much smaller than $f_{v} \sim 100$ commonly used as the basis of comparisons. These results obviously argue against the approach of taking the $v^{\text {th }}$ root of the total hindrance as a parameter (the Rusinov method [?]), since this brings together the underlying hindrance (expected since most transitions involve changes in seniority) with the systematic dependence on the $K$ forbiddeness. This approach will be discussed in more detail in a separate publication [? ].

\section{E3 transitions}

At this stage, the number of $E 3$ cases is insufficient to warrant a centroid analysis for different values of $\Delta \mathrm{K}$. The individual data are illustrated in Fig. 16, The open points correspond to several different regions, e.g. the transitional ${ }^{190}$ Os and ${ }^{192}$ Os nuclei and examples from the lighter ${ }^{130} \mathrm{Ba}$ and ${ }^{132} \mathrm{Ce}$ isotopes. A fit only to the filled points presented in Fig. 16 gives $F_{0}=3.7(+36-19)$ and $f_{0}=41(+11-9)$ (compare with Table C).

Table C

Fit parameters corresponding to the results of Fig.15.

\begin{tabular}{|c|c|c|c|}
\hline$\sigma \lambda$ & $F_{0}$ & $f_{0}$ & $\chi^{2}$ \\
\hline \multicolumn{4}{|c|}{ all points } \\
\hline$\overline{\mathrm{E} 1}$ & $2.0_{-15}^{+65} \times 10^{5}$ & $6.4_{-21}^{+32}$ & 2.2 \\
\hline M1 & $2.9^{+71} \times 10^{3}$ & $4.5^{+27}$ & 1.0 \\
\hline E2 & $1.2_{-7}^{+17} \times 10^{2}$ & $2.8_{-10}^{+15}$ & 1.2 \\
\hline \multicolumn{4}{|c|}{ selected points: see Fig. 15} \\
\hline E1 & $1.0_{-8}^{+37} \times 10^{5}$ & $12.5_{-62}^{+124}$ & 0.3 \\
\hline M1 & $1.7_{-3}^{+55} \times 10^{3}$ & $6.7_{-33}^{+65}$ & 0.3 \\
\hline E2 & $1.2_{-7}^{+17} \times 10^{2}$ & $3.2_{-15}^{+28}$ & 0.9 \\
\hline
\end{tabular}




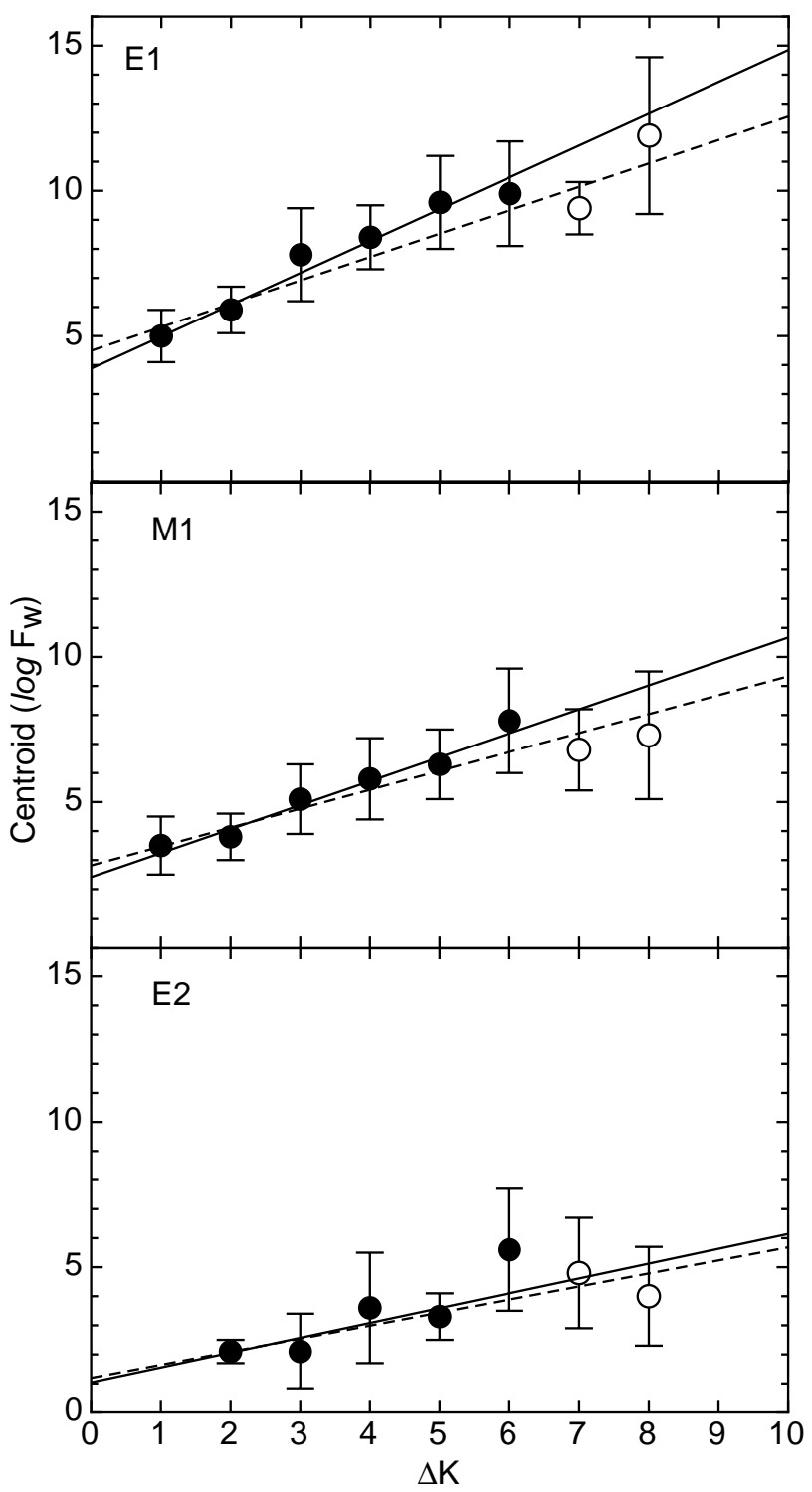

Fig. 15: Linear fits to eqn. 2 The dashed line includes all points, and open circles denote data excluded from the final fit (solid line), as discussed in the text.

\section{Coarse statistics}

Finally, we include here some observations about the data base and statistics in general. The decay of approximately 370 isomeric states and the strength of their numerous deexcitation decays ( $\sim 1050 \gamma$-ray transitions) are listed in the tables. Only $\sim$ $65 \%$ of all cases have firm spin and parity assignments, the remainder being uncertain, as summarized in Fig. 17, As can be seen from the statistics for the different multipolarities in Fig. 18, there are few cases known for the main multipolarities above $\Delta K=8$, and there is an almost uniform distribution for $E 1$ multipolarity up to $\Delta K=7$. One point to note is that there is a strong correlation between the $E 2$ and $M 1$ cases, since most represent branches from the same isomer to members of a rotational band based on a lower- $K$ state. Approximately $70 \%$ of the reported isomeric states have rotational bands associated with them. 


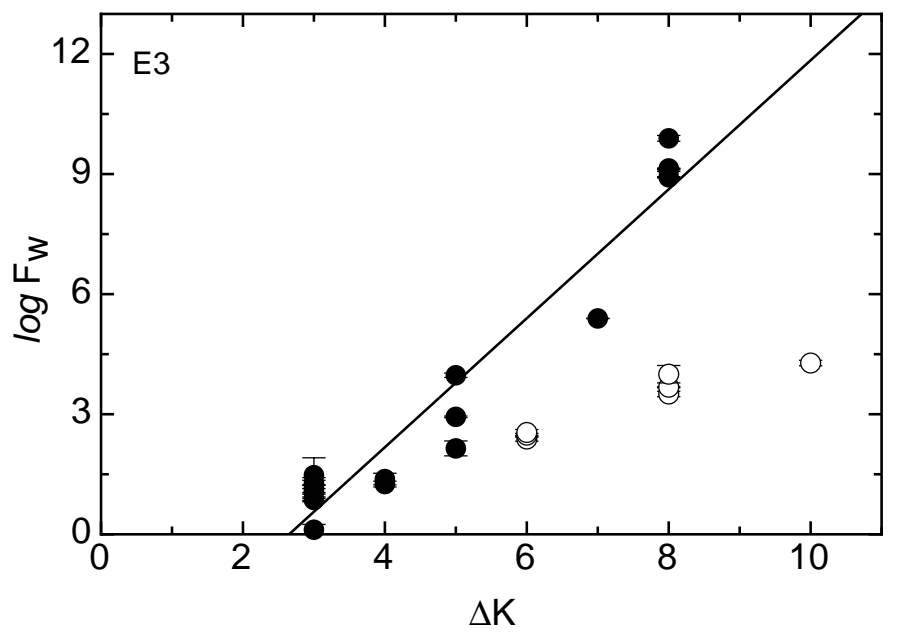

Fig. 16: $K$-forbidden strengths for $E 3$ transitions. The line corresponds to a fit to the points from the rare-earth region (filled symbols).

\section{Intrinsic states with sub-nanosecond lifetimes: experimental opportunities}

Significant progress has been made in recent years in the area of determination of sub-nanosecond lifetimes with the development of $\mathrm{LaBr}_{3}$ detectors. These are characterized by moderate energy resolution, but excellent timing characteristics, reaching into the tens of picosecond range. The use of such detectors in conjunction with high-resolution, multi-detector Ge arrays has opened up the possibility of isolating individual states in complex decay schemes, and determining lifetimes well below one nanosecond (see for example Refs. [? ? ]). A number of developments incorporating arrays of such detectors in new facilities are progressing [? ? ], but most (preparatory) measurements to date have been aimed at low-lying, collective states within rotational bands [? ? ] or at specific states in spherical nuclei [?].

Inspection of existing level schemes of well-deformed nuclei in the multi-quasiparticle regime, as outlined in this review, suggest that there are numerous prospects for the characterization of intrinsic states with lifetimes less than $1 \mathrm{~ns}$, the limit presently achieved (depending on energy and population) with most Ge arrays. Factors which contribute to this situation include the fact that the energy of the first transition in a high- $K$ rotational band is commonly $\sim 200 \mathrm{keV}$ or higher, comparable in many instances to the energy gap to the next, higher- $K$ intrinsic state. The net result of this situation is that in nuclei, such as ${ }^{174} \mathrm{Yb}$ for example [? ], the high-spin level sequences proceed through succession of high- $K$ intrinsic states and a few rotational band levels. While the lifetimes of some of the intrinsic states of ${ }^{174} \mathrm{Yb}$ have already been measured [? ], several others were not, leading to considerable ambiguity in the assignments and actual placements in the level scheme. This could be relieved if the lifetimes of the short-lived (less than $1 \mathrm{~ns}$ ) intrinsic states could be measured. There are about five examples of such states in the $\sim 13 \hbar$ to $\sim 21 \hbar$ spin region of ${ }^{174} \mathrm{Yb}$ alone [?] and many more short-lived intrinsic states, whose lifetimes are known as upper limits only, are listed in Table 1 .

\section{Scope and Policies}

As indicated above, there is a large body of information on isomeric states in deformed nuclei, and with emerging experimental techniques, this is likely to grow. The focus of the present review, however, is on those states that have been sufficiently well characterized in terms of spin, $\mathrm{K}$ value, and parity or at least limited ambiguity in assignment, and with well-defined decay branches. This is sufficient information to evaluate reliably the transition strengths. The tables include re-evaluated properties of $K$ isomers, 

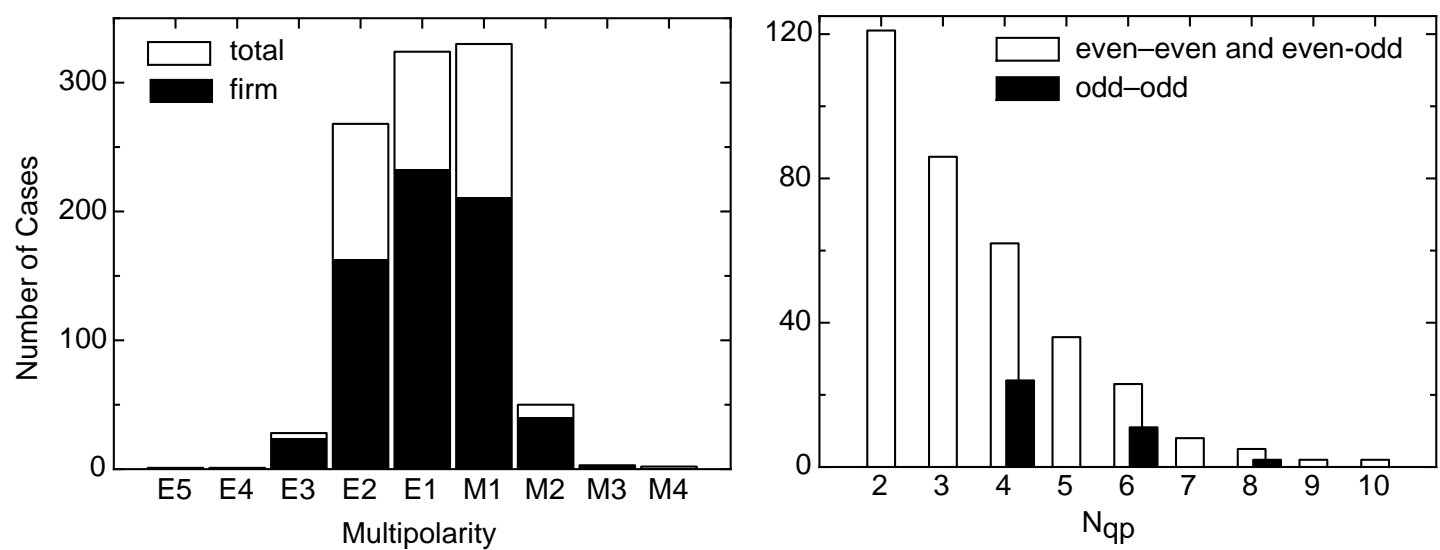

Fig. 17: Breakdown of the number of cases with respect to multipolarity and the seniority, $\mathrm{N}_{q p}$.

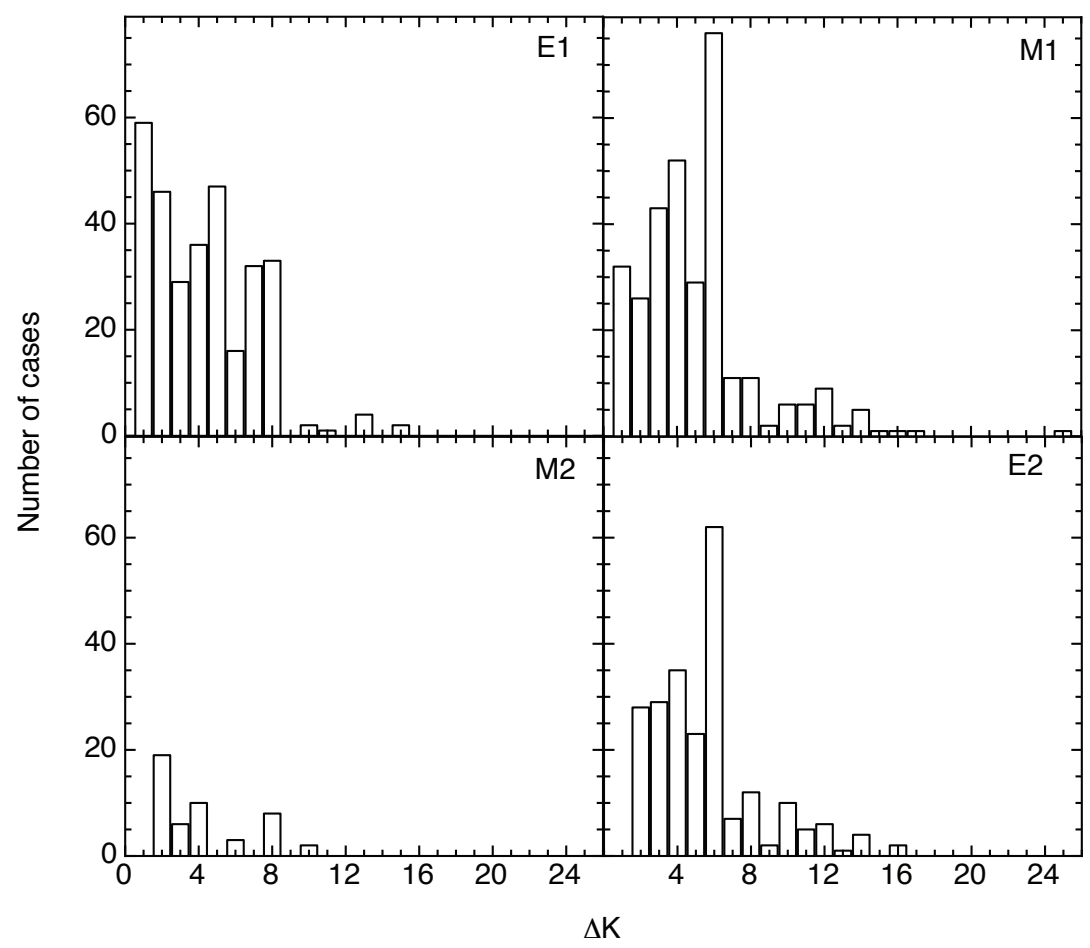

Fig. 18: Breakdown of the statistics for different multipolarities as a function of $\Delta K$. 
including half-lives, $\gamma$-ray transition multipolarities and intensities, and deduced widths and hindrances, as well as a table of assigned multi-quasiparticle configurations, and bibliography. In using these results, the reader is encouraged to refer to the original papers, particularly with respect to evaluations of the hindrances and to evidences needed for the configuration assignments, as well as with respect to the the nuclear structure interpretation and comparisons with nuclear models.

In the development of the present compilation, data available in the general purpose databases, such as the "Evaluated Nuclear Structure Data File" (ENSDF) [?] and the "Unevaluated Nuclear Data List" (XUNDL) [? ], as well as in the NubASE2012 evaluation [? ], were consulted. The tables include corrections for the (numerous) inconsistencies existing in those databases, and a large volume of additional data. The data are organized in ENSDF-formatted files and a number of computer programs, frequently used in nuclear data evaluation procedures for the ENSDF database, were applied.

In general, the quoted level energies were obtained from a least-squares fit to the corresponding $\gamma$-ray energies. The level quantum numbers and $\gamma$-ray transition multipolarities were inferred from the corresponding level schemes, as reported in the original articles and, where these were uncertain, they are placed in brackets. When the half-life of the isomer is known as a limit or approximate, the corresponding values for the widths and hindrances are also given as limits or approximate. In the cases of $\Delta I=0, \pm 1$ transitions with mixed multipolarities, only those for which the mixing ratios are well defined were included. In all other cases, the transitions were assumed to be pure dipole. The transition intensities are represented with the corresponding branching ratios and by normalizing to 100 units for the strongest branch, as adopted in ENSDF. In cases where the intensity of a particular branch was known as an upper limit, e.g. $I_{\gamma}<a$, a value of $I_{\gamma}=a / 2 \pm a / 2$ was assumed in calculating the corresponding widths and hindrances. Uncertainties were propagated using the python module "Uncertainties" [? ] and conversion coefficients were calculated using the BrICC code [? ]. Complete bibliographical references are included in Table 2 for each isomeric state. The NSR reference scheme is used and most of the references were retrieved from the NSR database [?].

\section{Relevant formulas and definitions}

The lifetime of an isomeric state is related to the total decay width, $\Gamma$, a linear sum of all partial decay widths ( $\gamma$ ray, conversion electrons, $\alpha$ decay, $\beta$ decay, fission, etc.), through the uncertainty relationship (in convenient units):

$$
\Gamma \times \tau=\hbar=0.6582 \times 10^{-15}[\mathrm{eV} \cdot \mathrm{sec}]
$$

where $\tau$ is the level mean life, which is related to the half-life as $\mathrm{T}_{1 / 2}=\ln 2 \times \tau$.

For an isomeric state with $N$ branches, predominantly $\gamma$ rays and internal conversion in the present cases, the partial $\gamma$-ray mean life of an individual transition $j, \tau_{\gamma}^{j}$, is given by:

$$
\tau_{\gamma}^{j}=\tau^{\exp } \times \frac{\sum_{k=1}^{N} I_{\gamma}^{k} \times\left(1+\alpha_{T}^{k}\right)}{I_{\gamma}^{j}},
$$

where $\tau^{\exp }$ is the experimental level mean life, $I_{\gamma}^{k}(k=1,2,3, \ldots, N)$ are the $\gamma$-ray intensities of the depopulating transitions and $\alpha_{T}^{k}$ are the total conversion coefficients. The corresponding partial $\gamma$-ray decay width, $\Gamma_{\gamma}^{j}$, can be deduced from equations 3 and 4 as:

$$
\Gamma_{\gamma}^{j}=\frac{\hbar}{\tau_{\gamma}^{j}}=\frac{\hbar}{\tau^{\exp }} \times \frac{I_{\gamma}^{j}}{\sum_{k=1}^{N} I_{\gamma}^{k} \times\left(1+\alpha_{T}^{k}\right)} .
$$


In the case of mixed multipolarity transitions, labeled $\sigma \lambda$ and $\sigma^{\prime} \lambda^{\prime}$ with $\lambda^{\prime}=\lambda+1$, where $\sigma \lambda$ is the multipolarity and $\lambda$ is the multipole order of the transition, the relationship between the mixing ratio $\delta\left(\sigma^{\prime} \lambda^{\prime} / \sigma \lambda\right)$ and the experimental transition probabilities and widths is:

$$
\delta^{2}\left(\sigma^{\prime} \lambda^{\prime} / \sigma \lambda\right)=\frac{I_{\gamma}\left(\sigma^{\prime} \lambda^{\prime}\right)}{I_{\gamma}(\sigma \lambda)}=\frac{\Gamma_{\gamma}\left(\sigma^{\prime} \lambda^{\prime}\right)}{\Gamma_{\gamma}(\sigma \lambda)} .
$$

Thus, for a mixed transition $j$, the partial $\gamma$-ray mean life and decay width for the individual components become:

$$
\begin{array}{ccc}
\tau_{\gamma}(\sigma \lambda)=\tau_{\gamma}^{j} \times\left(1+\delta^{2}\right) \quad \text { and } & \Gamma_{\gamma}(\sigma \lambda)=\Gamma_{\gamma}^{j} \times \frac{1}{1+\delta^{2}}, \\
\tau_{\gamma}\left(\sigma^{\prime} \lambda^{\prime}\right)=\tau_{\gamma}^{j} \times \frac{1+\delta^{2}}{\delta^{2}} \quad \text { and } \quad \Gamma_{\gamma}\left(\sigma^{\prime} \lambda^{\prime}\right)=\Gamma_{\gamma}^{j} \times \frac{\delta^{2}}{1+\delta^{2}},
\end{array}
$$

where $\tau_{\gamma}^{j}$ and $\Gamma_{\gamma}^{j}$ are determined from equations 4 and 5, respectively, and the total conversion coefficient for the mixed transition is obtained from the relation:

$$
\alpha_{T}^{k}=\frac{\alpha_{T}(\sigma \lambda)+\delta^{2} \times \alpha_{T}\left(\sigma^{\prime} \lambda^{\prime}\right)}{1+\delta^{2}} .
$$

Various alternative approaches are used in the literature for comparing $\gamma$-ray transition strengths, such as reduced $\gamma$-ray transition probabilities, $B_{\gamma}(\sigma \lambda) \downarrow$ (downward arrow indicates de-excitation probability), but these are simply related. For example,

$$
\frac{\tau_{W}}{\tau_{\gamma}}=\frac{B_{\gamma}(\sigma \lambda) \downarrow}{B_{s p}(\sigma \lambda) \downarrow}
$$

where $\tau_{W}$ and $B_{s p}(\sigma \lambda) \downarrow$ are the single-particle (Weisskopf) estimates for the partial $\gamma$-ray transition mean life and reduced transition probability, respectively. The required $B_{s p}(\sigma \lambda) \downarrow$ for electric $(\sigma \equiv E)$ transitions are given by:

$$
B_{s p}(E \lambda) \downarrow=\frac{1}{4 \pi} \times\left(\frac{3}{3+\lambda}\right)^{2} \times\left(1.2 \times A^{1 / 3}\right)^{2 \lambda} \quad\left[e^{2} \cdot f m^{2 \lambda}\right],
$$

and for magnetic $(\sigma \equiv M)$ transitions by:

$$
B_{s p}(M \lambda) \downarrow=\frac{10}{4 \pi} \times\left(\frac{3}{3+\lambda}\right)^{2} \times\left(1.2 \times A^{1 / 3}\right)^{2 \lambda-2} \quad\left[\mu_{N}^{2} \cdot f m^{2 \lambda-2}\right],
$$

where $e^{2}=1.439964485(33)[\mathrm{MeV} \cdot \mathrm{fm}]$ and $\mu_{N}^{2}=\left(e \hbar / 2 M_{p} c\right)^{2}=0.0159223489(13)\left[\mathrm{MeV} \cdot \mathrm{fm}^{3}\right][?]$.

The partial $\gamma$-ray decay width and mean life for a transition with an energy, $\mathrm{E}_{\gamma}$, and multipolarity, $\sigma \lambda$, are related to $B_{\gamma}(\sigma \lambda) \downarrow[?$ ]:

$$
\Gamma_{\gamma}(\sigma \lambda)=\frac{\hbar}{\tau_{\gamma}(\sigma \lambda)}=\frac{8 \pi \times(\lambda+1)}{\lambda \times[(2 \lambda+1) ! !]^{2}} \times\left(\frac{E_{\gamma}}{\hbar c}\right)^{2 \lambda+1} \times B_{\gamma}(\sigma \lambda) \downarrow .
$$

Furthermore, the strength $|M|^{2}$ of an individual transition in single-particle (Weisskopf) units (W.u.) is related to its widths, lifetimes and $B$ values, and to the inverse of its hindrance factor $\left(F_{W}\right)$ by:

$$
|M|^{2}(\text { W.u. })=\Gamma_{\gamma} / \Gamma_{W}=\tau_{W} / \tau_{\gamma}=B_{\gamma} \downarrow / B_{s p} \downarrow=1 / F_{W}
$$

For transitions that are (in principle) forbidden, the degree of $K$ forbiddenness, $v$, is defined as:

$$
v=\Delta K-\lambda
$$

where $\Delta K=\left|K_{i}-K_{f}\right|$ is the difference between the $K$ quantum numbers of the initial and final states, and $\lambda$ is the multipole order of the transition. The reduced hindrance per degree of $K$ forbiddenness is given by:

$$
f_{v}=F_{W}^{\frac{1}{v}}
$$


Table D

Values of the coefficients defined in sets of equations 17 and 18

\begin{tabular}{cccc}
\hline$\sigma \lambda$ & $a(\sigma \lambda)$ & $b(\sigma \lambda)$ & $c(\sigma \lambda)$ \\
\hline E1 & $9.75598 \mathrm{E}-15$ & $6.28848 \mathrm{E}-16$ & $6.44578 \mathrm{E}-02$ \\
E2 & $1.37398 \mathrm{E}-08$ & $8.16202 \mathrm{E}-10$ & $5.94043 \mathrm{E}-02$ \\
E3 & $2.94918 \mathrm{E}-02$ & $1.75194 \mathrm{E}-03$ & $5.94043 \mathrm{E}-02$ \\
E4 & $9.37820 \mathrm{E}+04$ & $5.89394 \mathrm{E}+03$ & $6.28473 \mathrm{E}-02$ \\
E5 & $4.17472 \mathrm{E}+11$ & $2.89263 \mathrm{E}+10$ & $6.92891 \mathrm{E}-02$ \\
& & & \\
M1 & $3.17627 \mathrm{E}-14$ & $5.68710 \mathrm{E}-14$ & $1.79049 \mathrm{E}+00$ \\
M2 & $4.47329 \mathrm{E}-08$ & $7.38146 \mathrm{E}-08$ & $1.65012 \mathrm{E}+00$ \\
M3 & $9.60171 \mathrm{E}-02$ & $1.58440 \mathrm{E}-01$ & $1.65012 \mathrm{E}+00$ \\
M4 & $3.05328 \mathrm{E}+05$ & $5.33029 \mathrm{E}+05$ & $1.74576 \mathrm{E}+00$ \\
M5 & $1.35917 \mathrm{E}+12$ & $2.61560 \mathrm{E}+12$ & $1.92470 \mathrm{E}+00$ \\
\hline
\end{tabular}

Both $F_{W}$ and $f_{v}$ quantities are used in evaluating the validity of multipolarity assignments, as well as in exploring nuclear structure effects and the relationship between the initial and final state configurations.

The required formulae for calculating $B$ values and lifetimes are widespread in the literature (see Refs. [? ? ], for example). For explicit multipolarities, one can use equations 11, 12 and 13 to obtain simpler expressions for electric transitions:

$$
\begin{array}{rlrl}
\tau_{W}(E \lambda)=a(E \lambda) \times \frac{1}{E_{\gamma}^{2 \lambda+1} \times A^{2 \lambda / 3}} & & {[s]} \\
B_{\gamma}(E \lambda) \downarrow & =b(E \lambda) \times \frac{1}{\tau_{\gamma} \times E_{\gamma}^{2 \lambda+1}} & & {\left[e^{2} \cdot f m^{2 \lambda}\right]} \\
B_{s p}(E \lambda) \downarrow & =c(E \lambda) \times A^{2 \lambda / 3} & & {\left[e^{2} \cdot f m^{2 \lambda}\right],}
\end{array}
$$

while for magnetic transitions:

$$
\begin{array}{cc}
\tau_{W}(M \lambda)=a(M \lambda) \times \frac{1}{E_{\gamma}^{2 \lambda+1} \cdot A^{(2 \lambda-2) / 3}} & {[s]} \\
B_{\gamma}(M \lambda) \downarrow=b(M \lambda) \times \frac{1}{\tau_{\gamma} \times E_{\gamma}^{2 \lambda+1}} & {\left[\mu_{N}^{2} \cdot f m^{2 \lambda-2}\right]} \\
B_{s p}(M \lambda) \downarrow=c(M \lambda) \times A^{(2 \lambda-2) / 3} & {\left[\mu_{N}^{2} \cdot f m^{2 \lambda-2}\right] .}
\end{array}
$$

The values for the coefficients $a(\sigma \lambda), b(\sigma \lambda)$ and $c(\sigma \lambda)$, listed in Table $\mathrm{D}$ were calculated using the recommended values for the fundamental constants [?], with $E_{\gamma}$ in $[M e V]$ and $\tau_{\gamma}(\sigma \lambda)$ in $[s]$.

\section{Acknowledgments}

This material is based upon work supported by the U.S. Department of Energy, Office of Science, Office of Nuclear Physics under contract No. DE-AC02-06CH11357 and by the Australian Research Council Discovery Program grants DP0986725, DP03445844 and DP140102986. The authors are thankful to Dr. R.V.F. Janssens and Dr. D.J. Hartley for the critical reading of the manuscript, and for many useful comments, and suggestions. 


\section{Explanation of Tables}

\section{TABLE1 1 Spectroscopic Information and K-forbidden Transition Probabilities.}

The nomenclature $2.5(3) E-3$ corresponds to $(2.5 \pm 0.3) \times 10^{-3}$.

${ }^{A} X \quad$ Mass number $(A)$ and chemical symbol $(X)$ of the nuclide studied.

$Z, N \quad$ Proton $(Z)$ and neutron $(N)$ numbers.

$\mathrm{E}_{i},[\mathrm{keV}] \quad$ Excitation energy of the isomeric state. Additional letters $(+X,+Y,+Z .$.$) indicate uncertain energy$ gaps relative to lower states. Uncertain assignments are indicated with $\dagger$.

$\mathrm{T}_{1 / 2}^{e x p} \quad$ Experimental half-life.

$\mathrm{K}_{i}^{\pi},[\hbar] \quad K$ value and parity $(\pi)$ of the isomer. The $K$ value coincides with the spin $\left(J_{i}\right)$ of the isomer, e.g $K_{i}=J_{i}$. Uncertain assignments are given in parentheses. Isomers labelled with $J^{\pi}=\ldots$ are not $K$ isomers, but arise from other mechanisms, such as rotation alignment, for example.

$\mathbf{J}_{f}^{\pi},[\hbar] \quad$ Spin value and parity $(\pi)$ of the final state. Uncertain assignments are indicated by parentheses.

$\mathrm{K}_{f}^{\pi},[\hbar] \quad K$ value and parity $(\pi)$ of the final state. Uncertain assignments are given in parentheses.

$\sigma \lambda \quad$ Gamma-ray transition multipolarity.

$\mathrm{E}_{\gamma},[\mathrm{keV}] \quad$ Gamma-ray transition energy. Transitions that are not observed experimentally, but are required by coincidence relationships, are given in brackets. Uncertain assignments are indicated with $\dagger$.

$\mathrm{I}_{\gamma} \quad$ Relative gamma-ray transition intensity normalized to 100 units for the strongest branch.

$\alpha_{T} \quad$ Total electron conversion coefficient calculated using the BRICC program [? ].

$\Gamma_{\gamma},[\mathrm{eV}] \quad$ Partial gamma-ray decay width.

$\mathrm{F}_{W} \quad$ Weisskopf hindrance factor.

$v \quad$ The degree of $\mathrm{K}$ forbiddenness.

$f_{v} \quad$ Reduced hindrance per degree of $\mathrm{K}$ forbiddenness. Values deduced with uncertain $\mathrm{K}^{\pi}$ and/or $\mathrm{J}^{\pi}$ values are indicated by italics. 
TABLE 2.

${ }^{A} X$

Z, N

References

$\mathrm{E}_{i},[k e V]$

$\mathrm{T}_{1 / 2}^{\exp }$

$\mathrm{K}^{\pi},[\hbar]$

Configuration
Mass number $(A)$ and chemical symbol $(X)$ of the nuclide studied.

Proton $(Z)$ and neutron $(N)$ numbers of the nuclide studied.

Bibliography given using the NSR reference key numbers [?].

Excitation energy of the isomeric state. Tentative assignments are indicated with $\dagger$. Additional letters $(+X,+Y,+Z .$.$) indicate uncertain energy gaps relative to lower states.$

Experimental half-life.

$K$ value and parity $(\pi)$ of the isomer. Uncertain assignments are indicated in brackets.

The addition of an asterisk $(*)$ indicates that an associated rotational band has been observed. Also, states labelled with $J^{\pi}=\ldots$ are not $K$ isomers, but arise from other mechanisms, such as rotation alignment, for example.

The isomer configuration using the Nilsson quantum numbers given separately for the neutrons and/or protons. Tentative assignments are indicated with $\dagger$. 


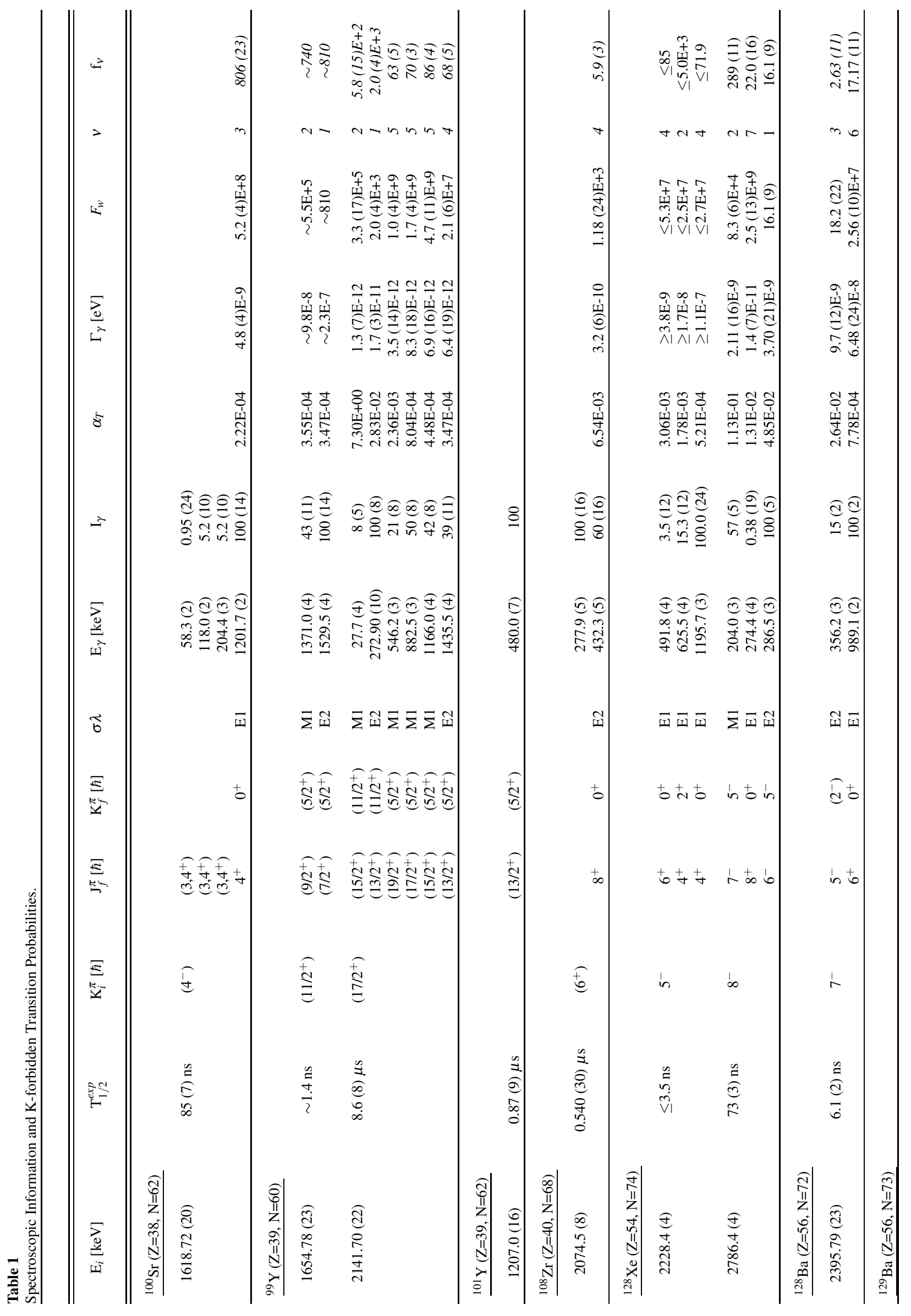




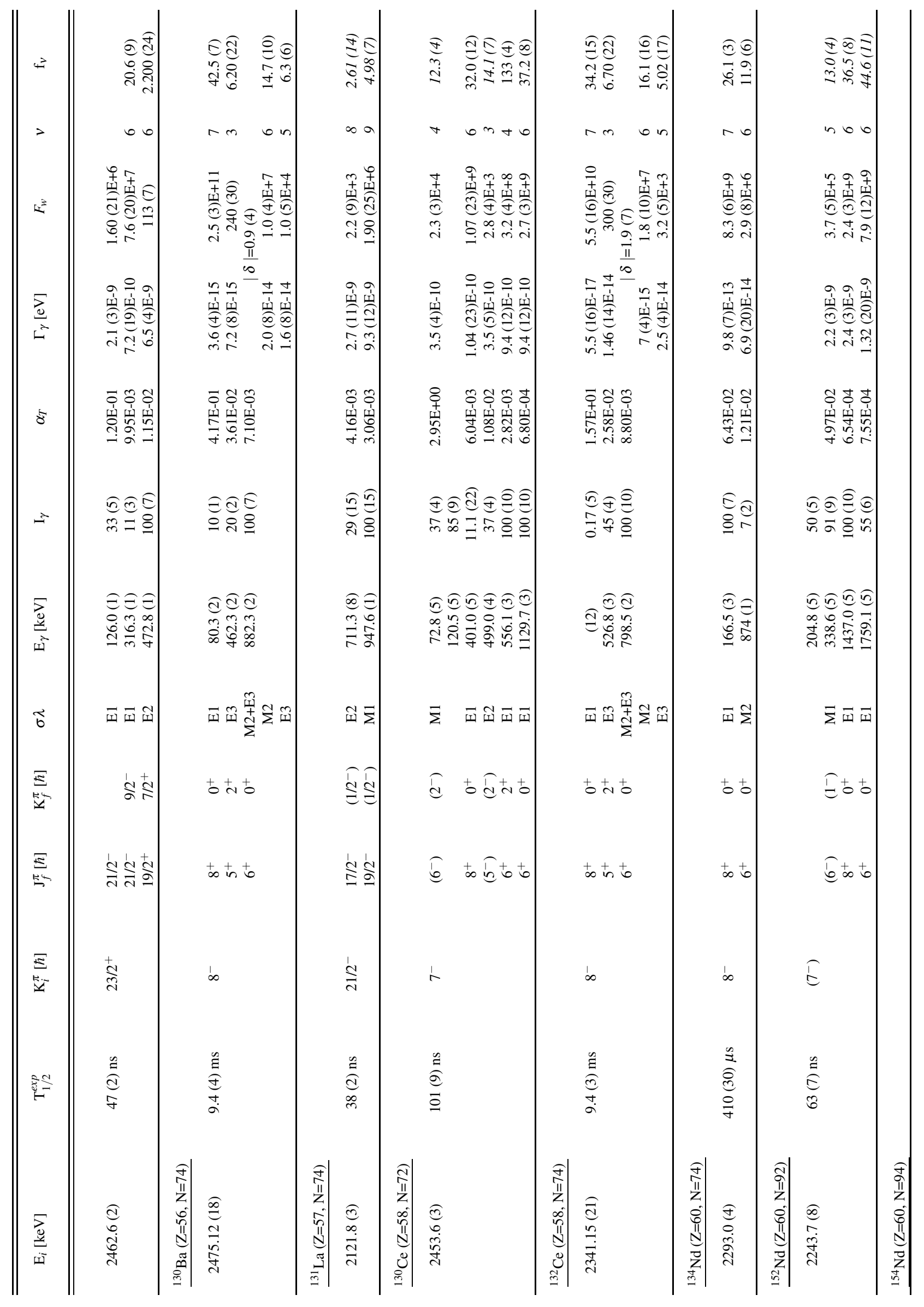




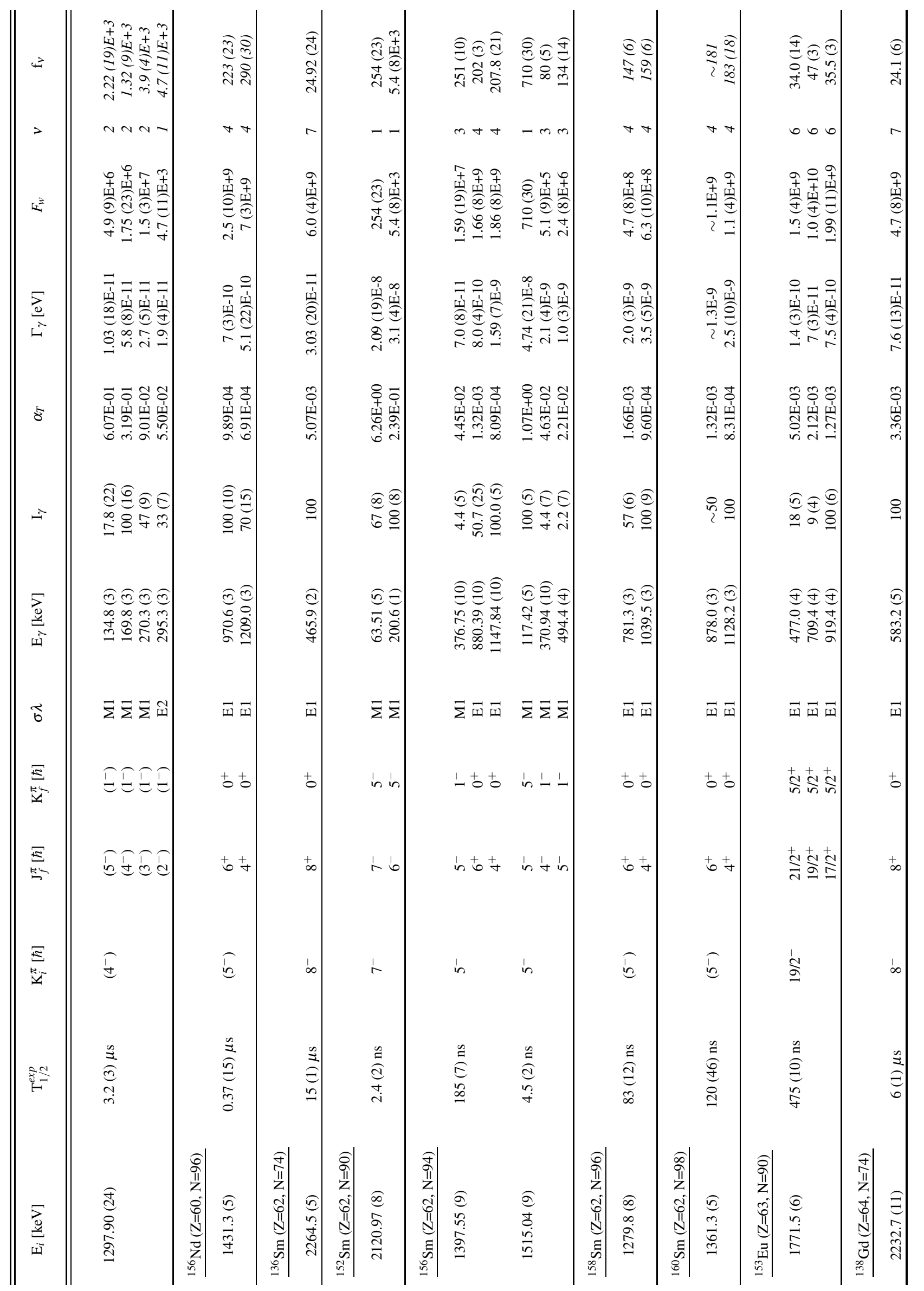




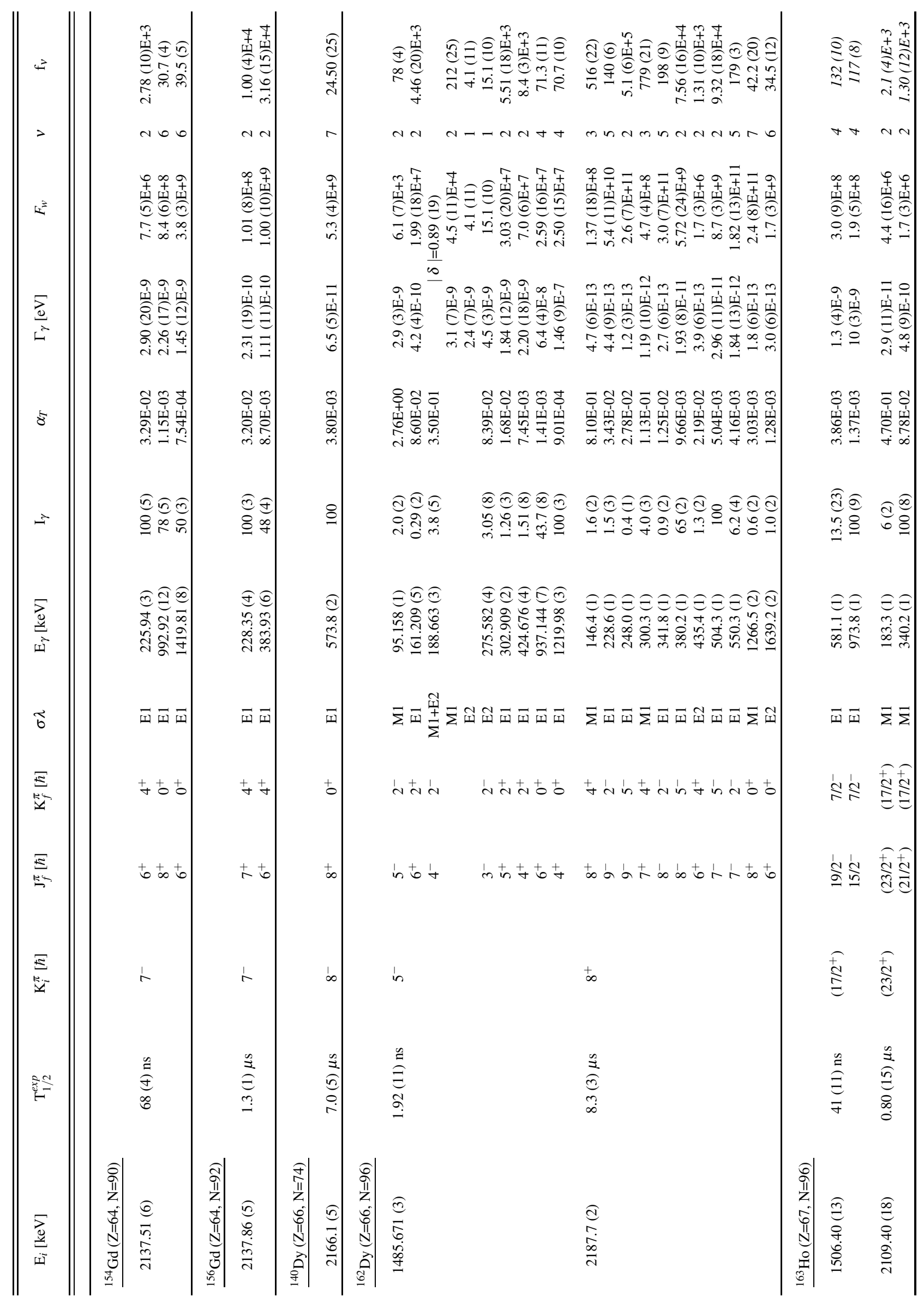




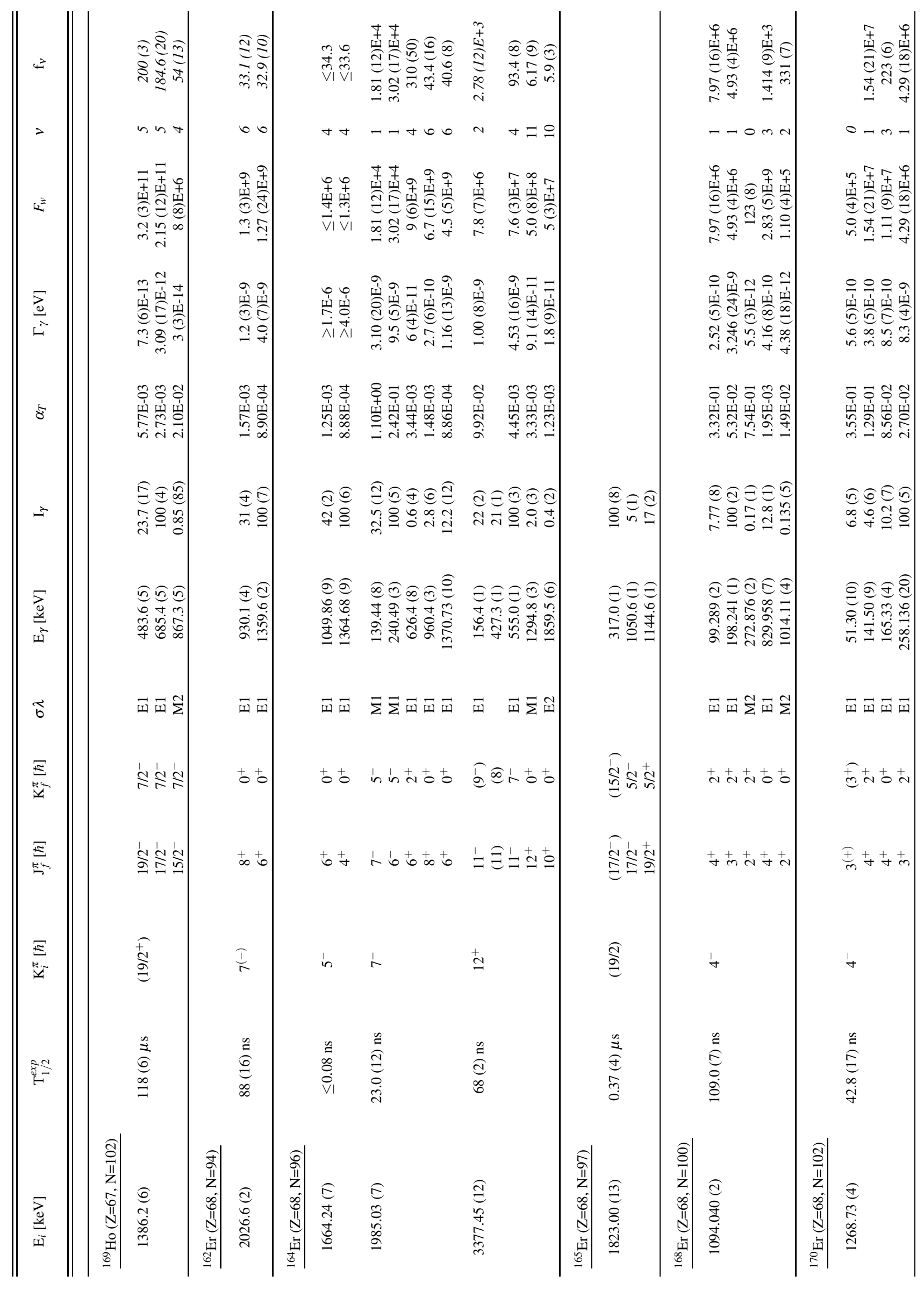




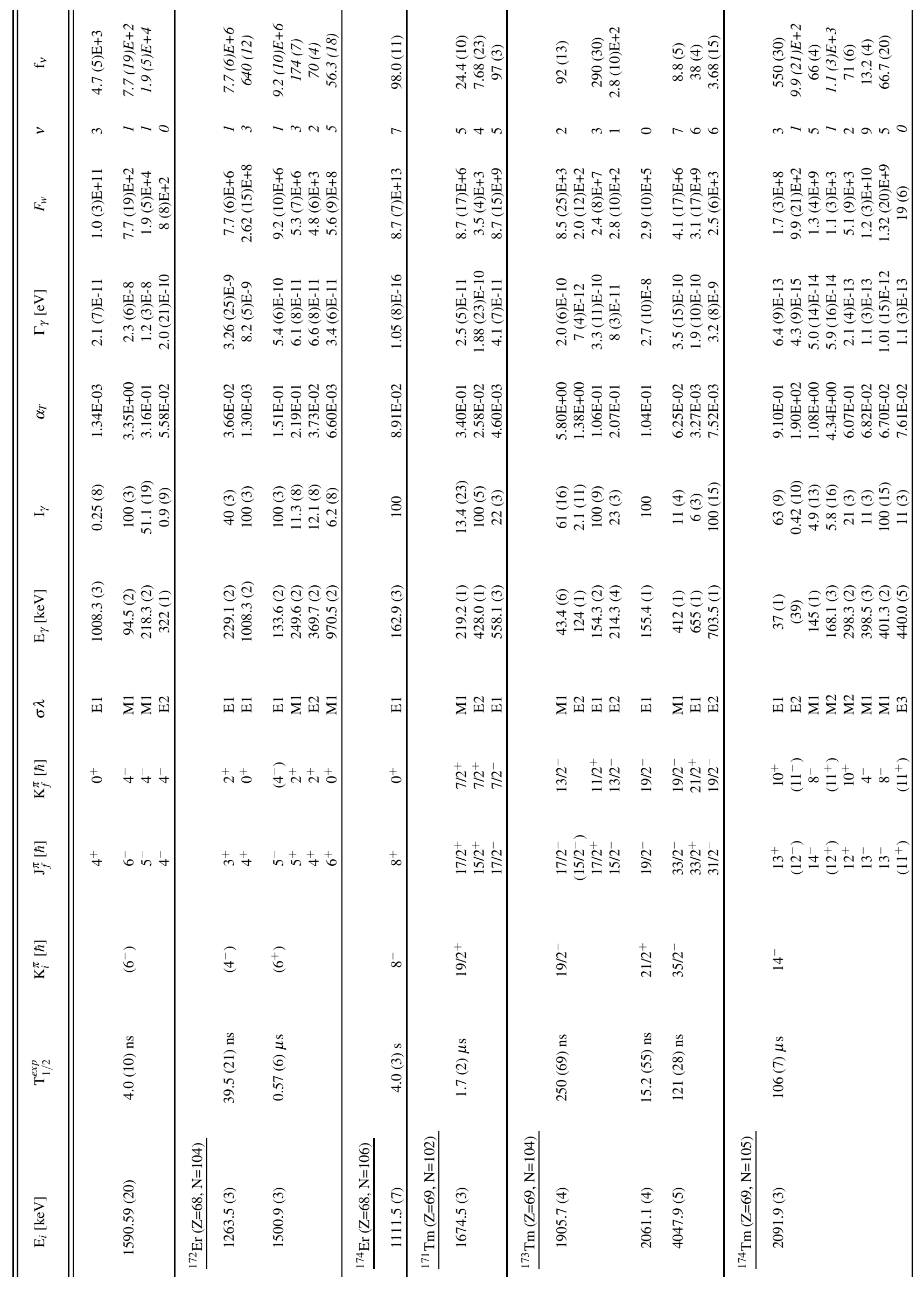




\begin{tabular}{|c|c|c|c|c|c|c|c|c|c|c|c|}
\hline$\rightarrow$ & 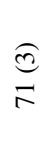 & & 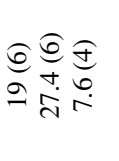 & 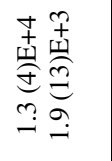 & & 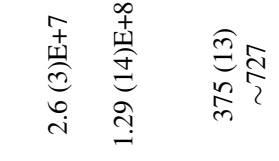 & 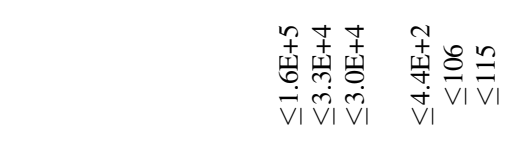 & 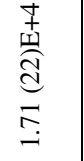 & & 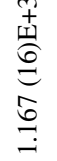 & 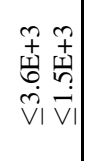 \\
\hline$>$ & ナ & & $m m n$ & $n-0$ & & $-\quad-0 \quad+\theta$ & $000--10 \mathrm{Nm}$ & -0 & & m & -- \\
\hline$x^{3}$ & 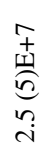 & & 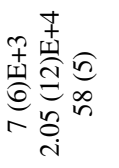 & 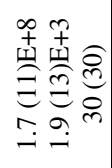 & & 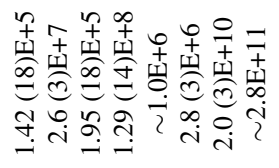 & 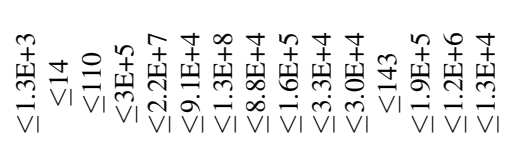 & 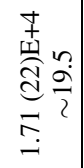 & & 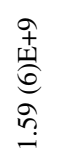 & 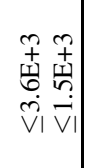 \\
\hline 竞 & 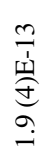 & & 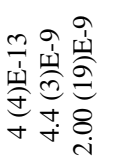 & 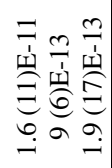 & & 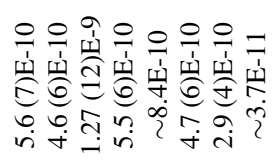 & 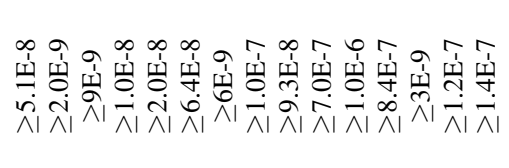 & 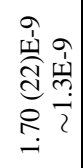 & & 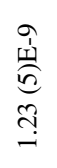 & 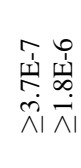 \\
\hline$\S$ & 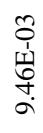 & & 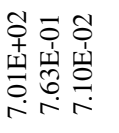 & 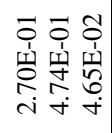 & & 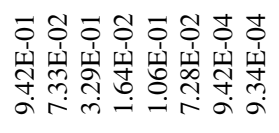 & 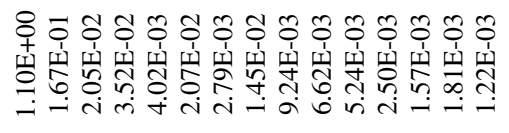 & 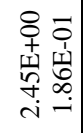 & & 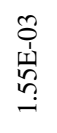 & 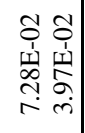 \\
\hline$\Rightarrow$ & $\widehat{0}$ & & 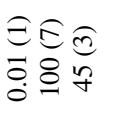 & 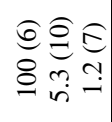 & & 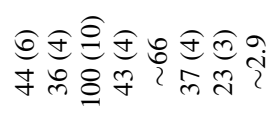 & 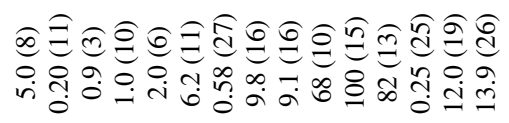 & $8 \frac{n}{2}$ & & $\stackrel{8}{8}$ & 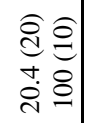 \\
\hline 恿 & 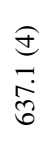 & & 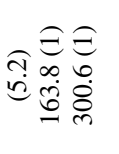 & 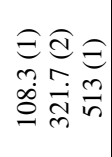 & & 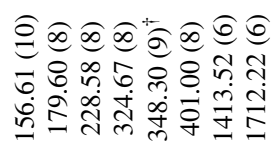 & 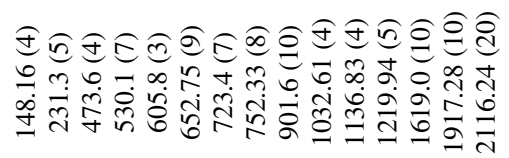 & 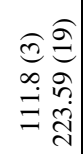 & & 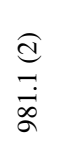 & 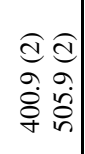 \\
\hline$\widetilde{b}$ & ్ㅗ & & $\bar{\Sigma} \bar{\Sigma} \tilde{I}$ & 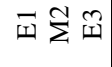 & & 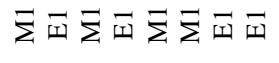 & 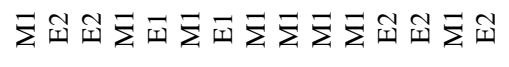 & $\bar{\Sigma} \widetilde{I}$ & & $\overline{1}$ & $\bar{\Sigma} \bar{\Sigma}$ \\
\hline $\begin{array}{l}E \\
E \\
E \\
y\end{array}$ & $\infty$ & & $\frac{\sqrt{n}}{1} \mathfrak{N}$ & 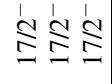 & & m & $\stackrel{+}{m} \stackrel{+}{m} \stackrel{+}{m} \stackrel{+}{\sim} \stackrel{+}{\sim} \stackrel{+}{\sim} b^{+} 0^{+}$ & in in & & to & $\dot{z}+$ \\
\hline $\begin{array}{ll}E \\
E\end{array}$ & $\stackrel{1}{\simeq}$ & & 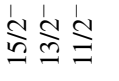 & 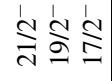 & & 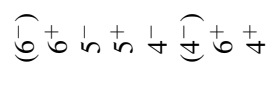 & 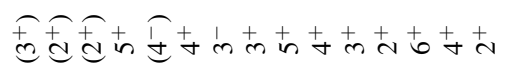 & 6 in & & + & 6 in \\
\hline $\begin{array}{l}E \\
\pm \\
\pm\end{array}$ & & & $\frac{\sqrt{2}}{2}$ & 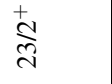 & & in & $\stackrel{+}{+}$ & $r$ & & $\dot{t}$ & 6 \\
\hline$\stackrel{\mathrm{m}}{\mathrm{B}}=$ & & & 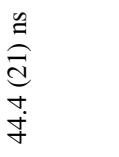 & 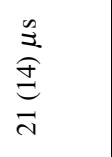 & & 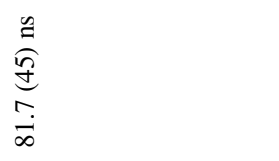 & 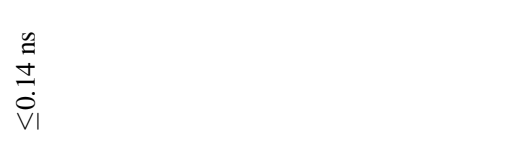 & $\underset{\substack{\infty \\
ٍ}}{\stackrel{0}{\infty}}$ & & 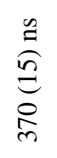 & 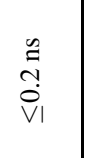 \\
\hline 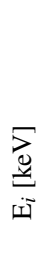 & & 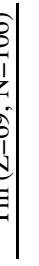 & 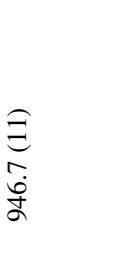 & 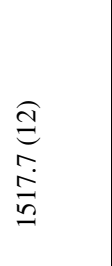 & 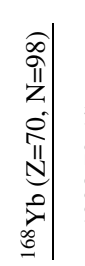 & 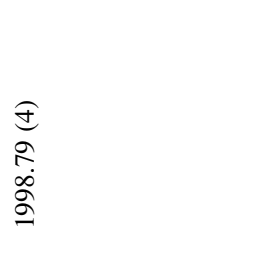 & 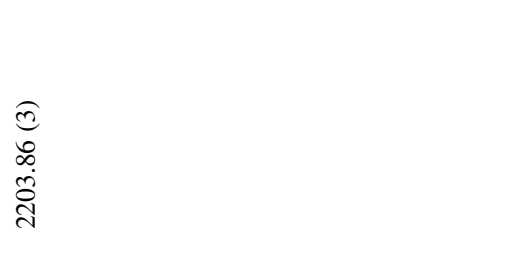 & 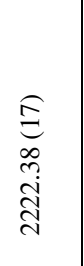 & 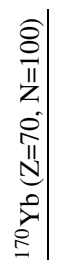 & 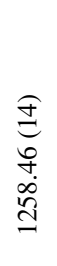 & 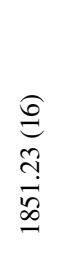 \\
\hline
\end{tabular}




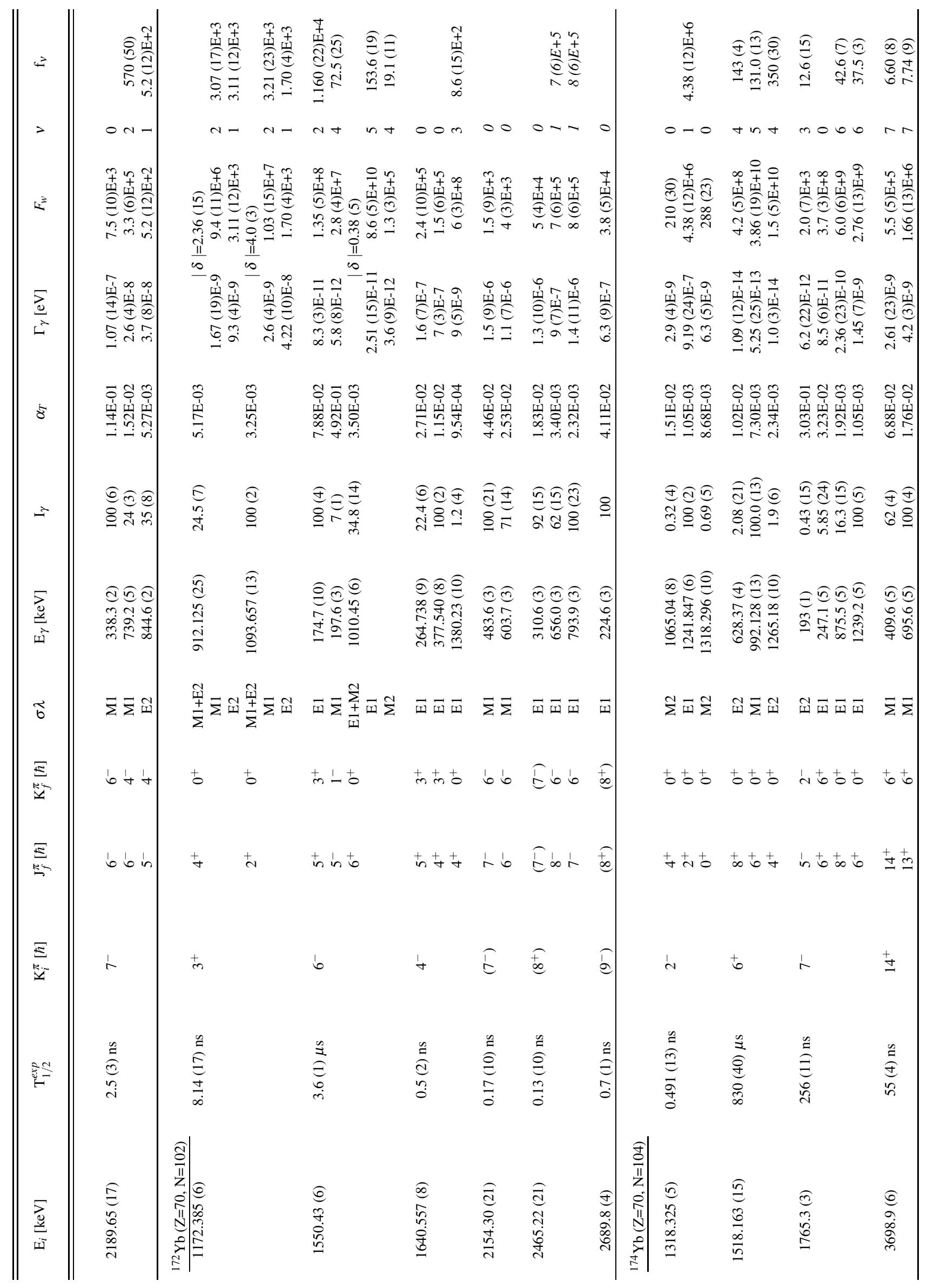




\begin{tabular}{|c|c|c|c|c|c|c|c|c|c|c|c|c|}
\hline 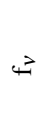 & 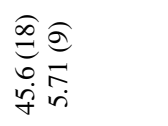 & & 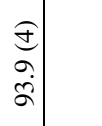 & 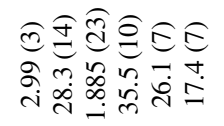 & & & & 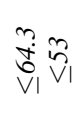 & & 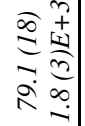 & 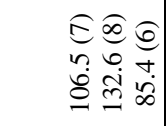 & 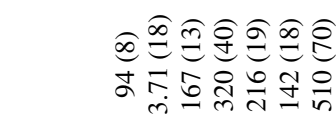 \\
\hline$>$ & 06 & & $r$ & $\cong n=0 n t$ & 0 & 0 & $\circ$ & $N-$ & 0 & $\forall-1$ & $n n t$ & $m 0 m+m n t$ \\
\hline$x^{3}$ & 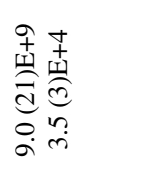 & & 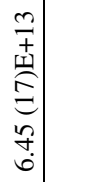 & 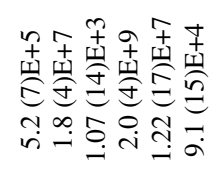 & 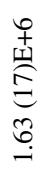 & $\begin{array}{l}0 \\
+ \\
1 \\
\infty \\
\infty \\
\text { VI }\end{array}$ & $\begin{array}{l}\text { तิ } \\
\text { V। }\end{array}$ & 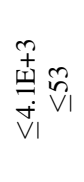 & 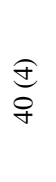 & 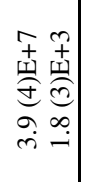 & 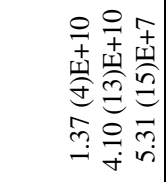 & 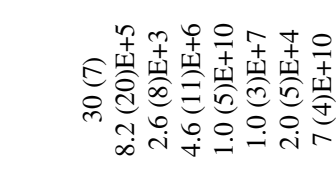 \\
\hline 胥 & 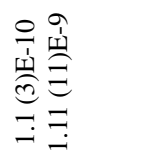 & & 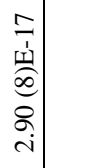 & 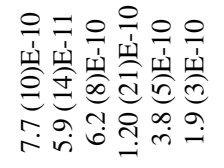 & 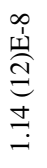 & $\begin{array}{l}\stackrel{i}{1} \\
\text { ले } \\
\stackrel{\lambda}{\lambda}\end{array}$ & $\begin{array}{l}\infty \\
\text { ஸे } \\
\text { ஸे } \\
\dot{\lambda}\end{array}$ & 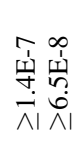 & 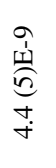 & 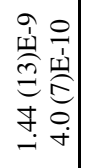 & 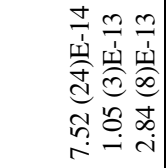 & 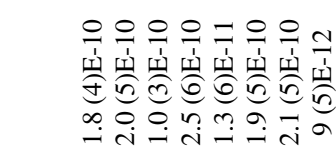 \\
\hline 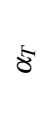 & 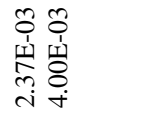 & & 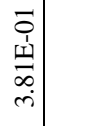 & 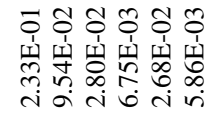 & 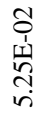 & 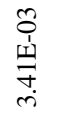 & 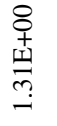 & 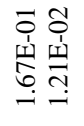 & 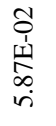 & 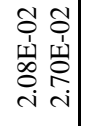 & 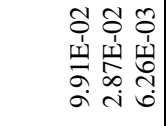 & 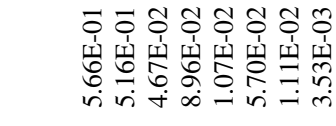 \\
\hline$\Rightarrow$ & 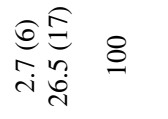 & & 8 & 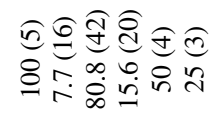 & 8 & 8 & 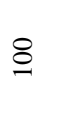 & $\begin{array}{l}\sqrt[6]{6} 5 \\
85 \\
0\end{array}$ & 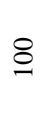 & 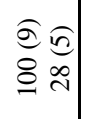 & 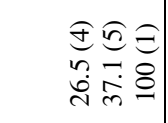 & 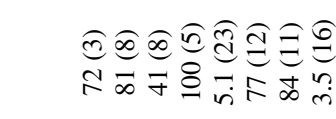 \\
\hline 岔 & 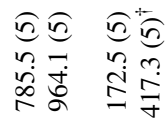 & & $\begin{array}{l}0 \\
\hat{\sigma} \\
\alpha \\
\check{\alpha}\end{array}$ & 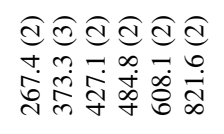 & $\begin{array}{l}\tilde{n} \\
\infty \\
\infty \\
\stackrel{i}{1}\end{array}$ & $\begin{array}{l}\sqrt[6]{0} \\
0 \\
0 \\
0 \\
0\end{array}$ & 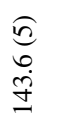 & 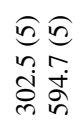 & $\begin{array}{l}\sqrt{c} \\
\infty \\
\infty \\
\infty\end{array}$ & 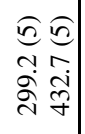 & 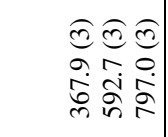 & 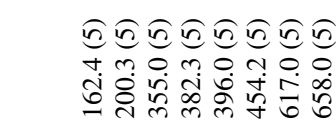 \\
\hline$\widetilde{b}$ & 피꼬 & & 피 & $\bar{\Sigma} \bar{\Sigma} \tilde{I} \bar{\Sigma} \bar{\Sigma} \tilde{I}$ & $\vec{\Psi}$ & $\overrightarrow{\text { ㅍ }}$ & $\bar{\Sigma}$ & $\bar{\Sigma} \tilde{y}$ & 포 & 포쇠 & $\bar{\Sigma} \bar{\Sigma} \mathbb{I}$ & 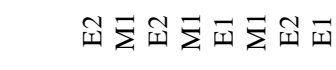 \\
\hline $\begin{array}{l}E \\
E \\
E\end{array}$ & $\therefore+\frac{i}{d} \stackrel{a}{=}$ & & to & ナたナとだ & $\stackrel{+}{=}$ & \pm & $\stackrel{f}{n}$ & $\underbrace{f}_{0}$ & 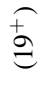 & 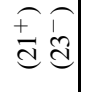 & 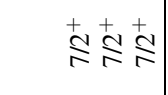 & ${ }_{\infty}^{+}+{ }^{+}+\infty{ }^{+}{ }_{\infty}^{+}+\infty$ \\
\hline$E_{-}^{E}$ & 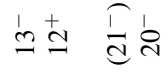 & & $+\infty$ & 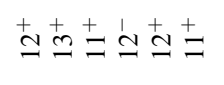 & $\stackrel{+}{\longrightarrow}$ & \pm & $\stackrel{f}{n}$ & $\stackrel{F}{\infty} \underset{E}{=}$ & $\stackrel{\Im}{\approx}$ & 㢳筞 & 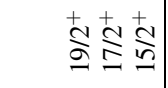 & 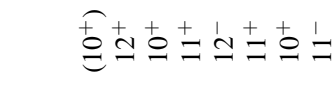 \\
\hline $\begin{array}{l}E \\
\Sigma\end{array}$ & $\begin{array}{l}\widehat{\tilde{I}} \\
\tilde{d}\end{array}$ & & $\infty$ & $\stackrel{+}{m}$ & \pm & $\stackrel{f}{i n}$ & $\stackrel{f}{\mathscr{E}}$ & $\stackrel{\Im}{\stackrel{2}{2}}$ & $\frac{\bar{c}}{\bar{d}}$ & డ్d & 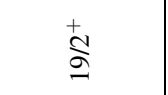 & $\stackrel{+}{\simeq}$ \\
\hline s= & 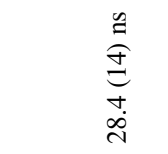 & & \begin{tabular}{l}
$\stackrel{\infty}{0}$ \\
\multirow{2}{*}{} \\
$\Xi$
\end{tabular} & 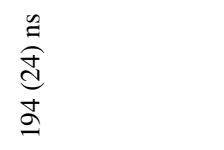 & 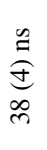 & 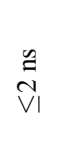 & $\begin{array}{l}\stackrel{y}{a} \\
\vec{V} \mid\end{array}$ & $\begin{array}{l}\tilde{y} \\
\vec{V} \mid\end{array}$ & $\begin{array}{l}\stackrel{a}{\varrho} \\
\stackrel{\Xi}{\sigma}\end{array}$ & 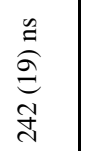 & 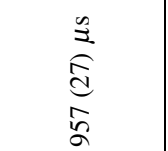 & $\begin{array}{l}\frac{n}{2} \\
\hat{E} \\
\frac{m}{0}\end{array}$ \\
\hline 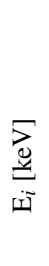 & 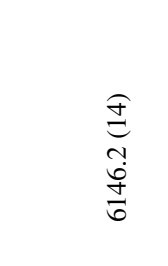 & 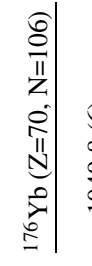 & 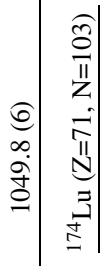 & 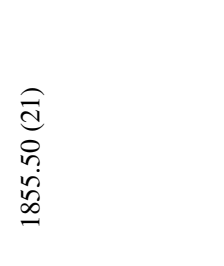 & $\begin{array}{l}\hat{e} \\
\text { an } \\
\text { òं } \\
\text { i }\end{array}$ & $\frac{\widehat{\infty}}{\stackrel{\infty}{\vec{d}}}$ & $\begin{array}{l}\hat{0} \\
\substack{n \\
n \\
\infty \\
i \\
i}\end{array}$ & 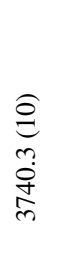 & \begin{tabular}{l}
$\widehat{\Xi}$ \\
\multirow{+}{+}{} \\
$\infty$ \\
$\stackrel{+}{+}$
\end{tabular} & 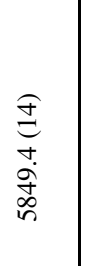 & 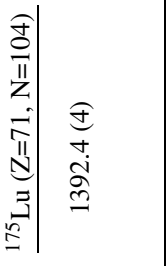 & 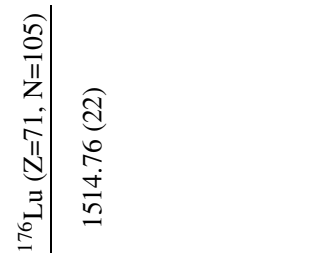 \\
\hline
\end{tabular}




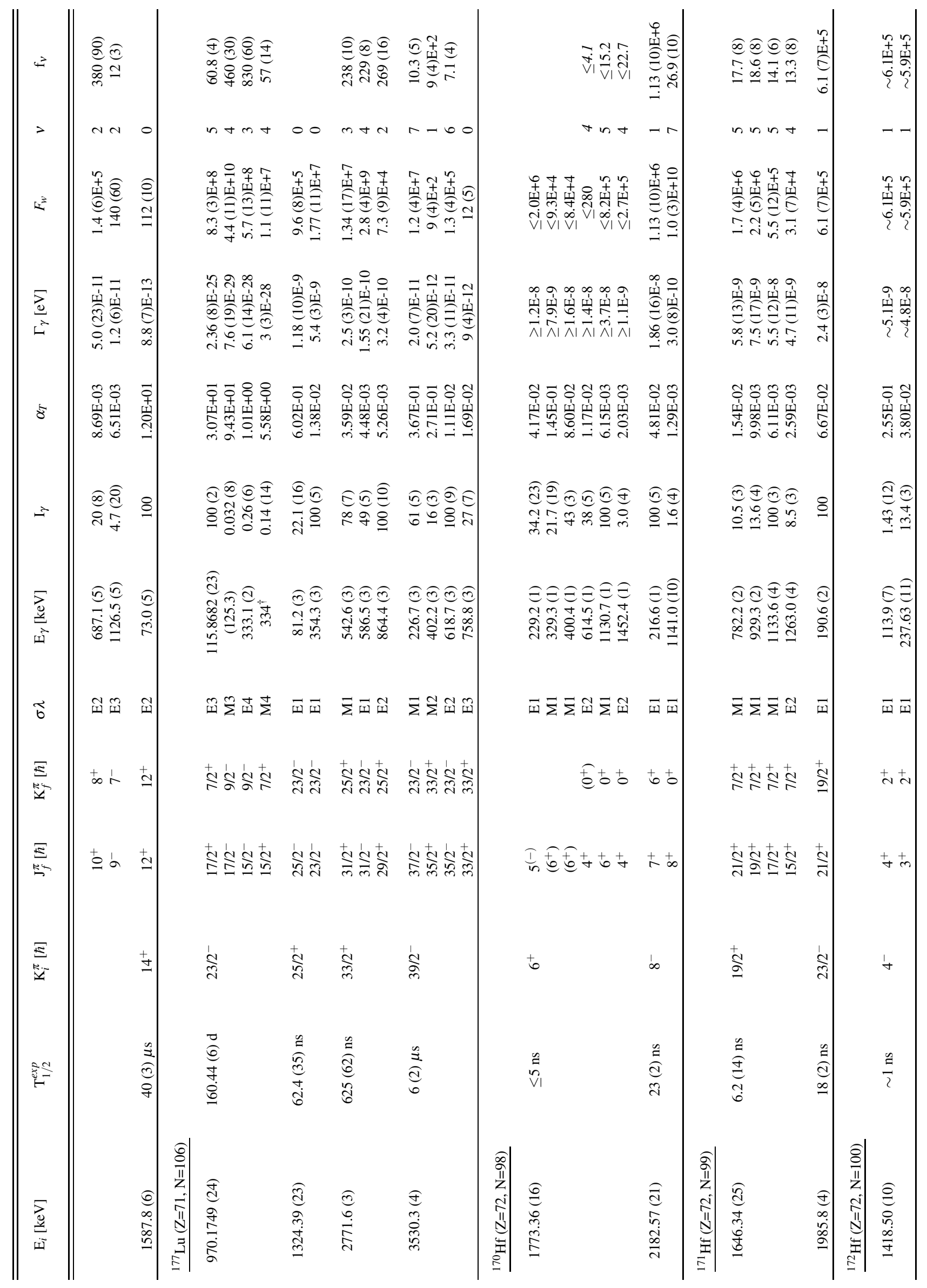




\begin{tabular}{|c|c|c|c|c|c|c|c|c|c|}
\hline$\rightarrow$ & 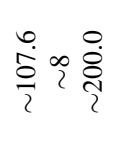 & 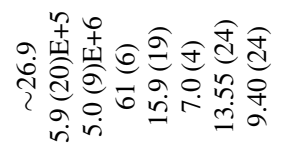 & 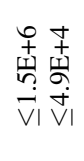 & 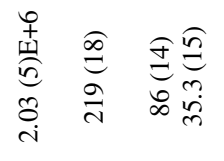 & 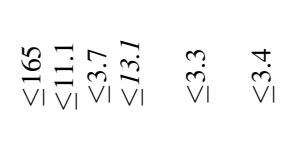 & 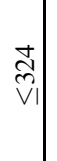 & & 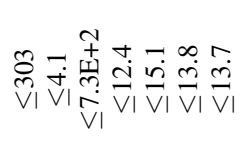 & 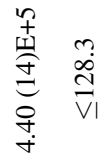 \\
\hline$>$ & $m \mathrm{Nm}$ & $m--m a t n \theta$ & -- & -OMONI & $m n+n=0$ & - & & $N+-\operatorname{nn} n t$ & - \\
\hline$x^{3}$ & 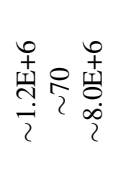 & 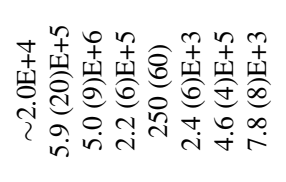 & 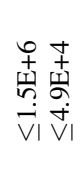 & 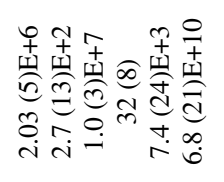 & 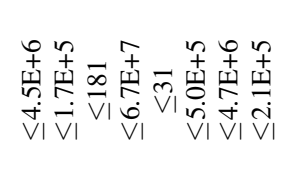 & ज़ & & 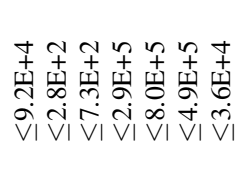 & 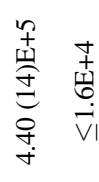 \\
\hline$\frac{D_{0}}{s_{2}^{2}}$ & 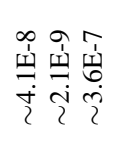 & 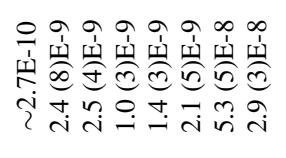 & 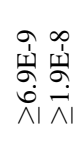 & 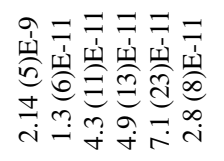 & 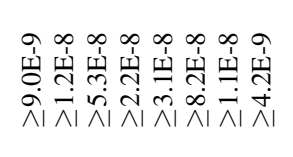 & 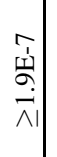 & & 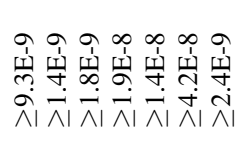 & 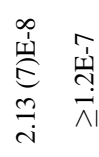 \\
\hline$\S$ & 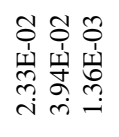 & 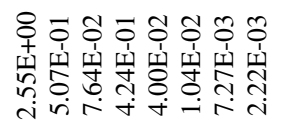 & 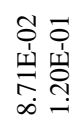 & 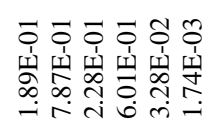 & 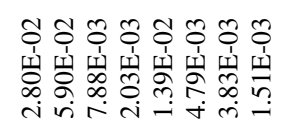 & $\begin{array}{l}8 \\
+ \\
\text { 站 } \\
\text { - } \\
-1\end{array}$ & & 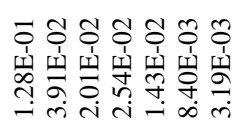 & 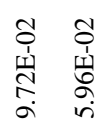 \\
\hline$\Rightarrow$ & 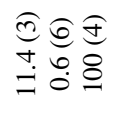 & 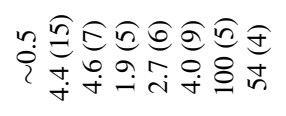 & 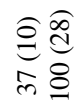 & 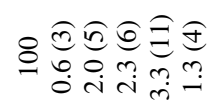 & 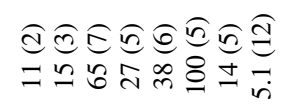 & $\approx$ & & 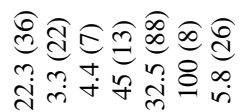 & $\stackrel{8}{\circ}$ \\
\hline 鹰 & 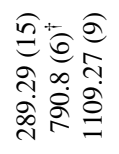 & 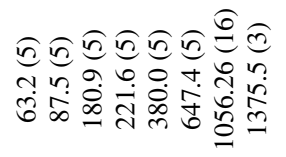 & 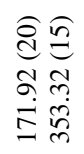 & 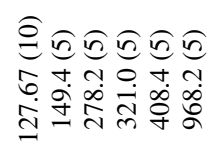 & 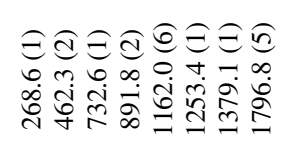 & 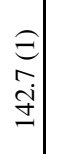 & & 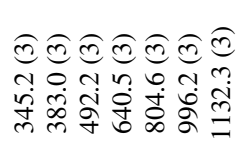 & $\begin{array}{l}\hat{0} \\
\infty \\
\dot{0} \\
\underline{0}\end{array}$ \\
\hline$\widehat{b}$ & 포 芝可 & 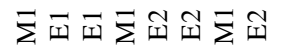 & $\bar{ㅍ} \bar{\Sigma}$ & 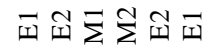 & 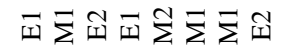 & $\bar{\Sigma}$ & & 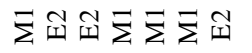 & 피 \\
\hline 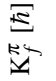 & さむち & さナナナさざすすす & +2 & $56+5+t 0$ & 施古导 t t & $\stackrel{+}{=}$ & & 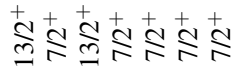 & 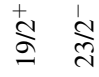 \\
\hline $\begin{array}{l}E \\
E\end{array}$ & $\begin{array}{r}+ \\
+ \\
\end{array}$ & 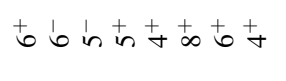 & to in & 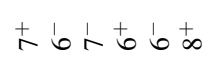 & 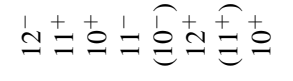 & $\stackrel{+}{2}$ & & 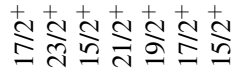 & $\stackrel{+}{\stackrel{N}{\sim}} \underset{\sim}{\stackrel{N}{N}}$ \\
\hline $\begin{array}{l}E \\
E \\
\Sigma\end{array}$ & & 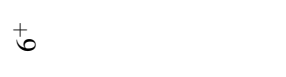 & 6 & $\infty$ & $\stackrel{+}{\beth}$ & $\stackrel{+}{ \pm}$ & & $\stackrel{9}{2}$ & $\stackrel{\text { I }}{\stackrel{\lambda}{7}}$ \\
\hline$\overbrace{\overrightarrow{3}=}^{2}$ & & 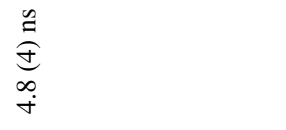 & 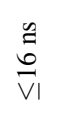 & 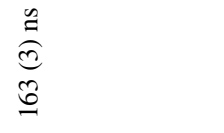 & $\begin{array}{c}\tilde{\Xi} \\
\tilde{V} \mid\end{array}$ & $\begin{array}{c}\tilde{a} \\
\vec{\nabla} \mid\end{array}$ & & $\begin{array}{l}\tilde{a} \\
\stackrel{n}{v} \mid\end{array}$ & 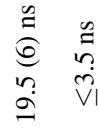 \\
\hline 敢 & & $\begin{array}{l}\widehat{\Xi} \\
o \\
0 \\
\dot{0} \\
0 \\
0\end{array}$ & 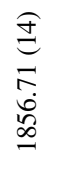 & 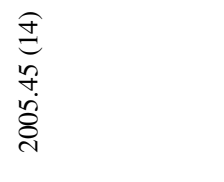 & 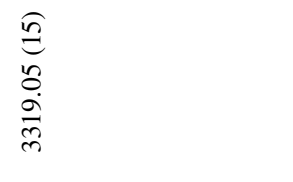 & 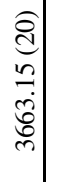 & 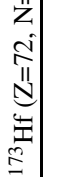 & 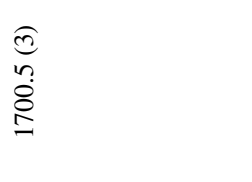 & 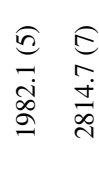 \\
\hline
\end{tabular}




\begin{tabular}{|c|c|c|c|c|c|c|}
\hline$\rightarrow$ & & 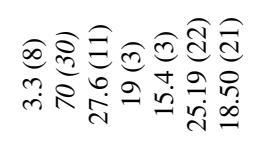 & 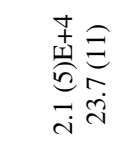 & 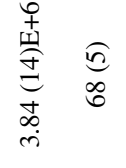 & 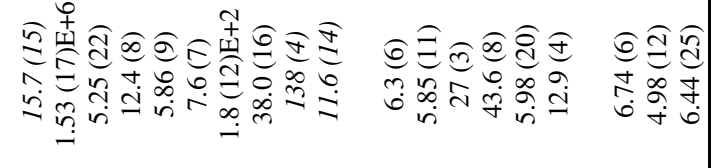 & 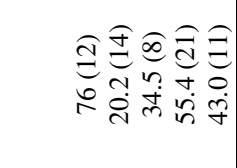 \\
\hline$>$ & & 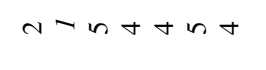 & $0-i n$ & -or & 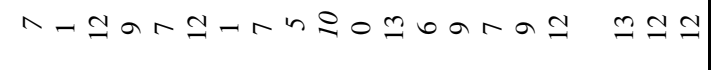 & ton in 0 \\
\hline$x^{3}$ & & 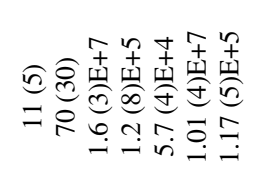 & 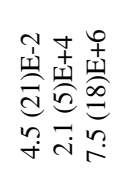 & 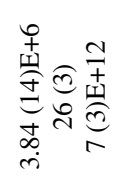 & 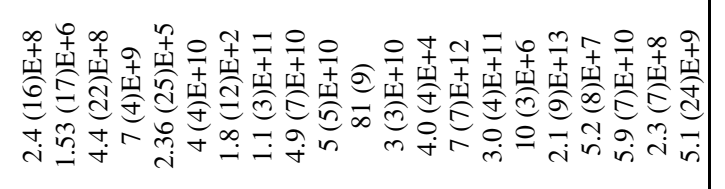 & 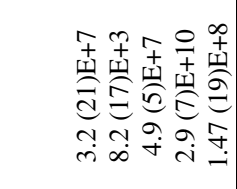 \\
\hline 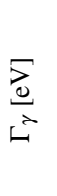 & & 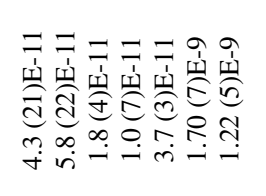 & 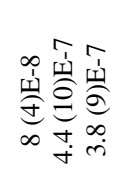 & 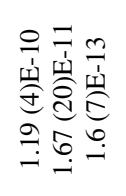 & 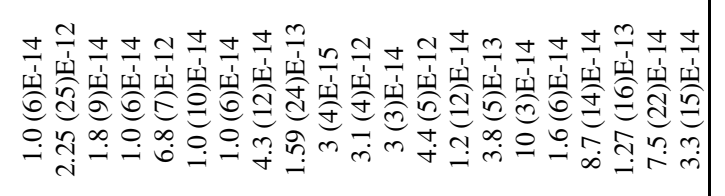 & 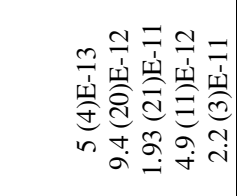 \\
\hline 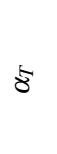 & & 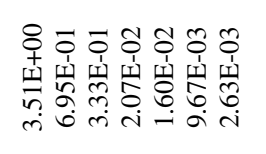 & 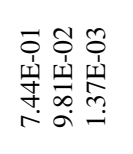 & 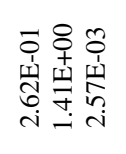 & 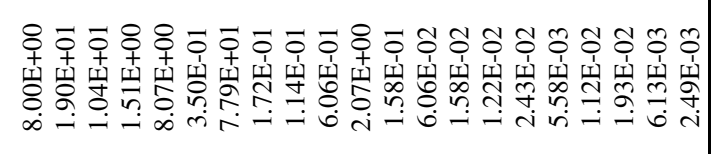 & 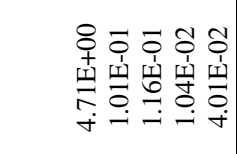 \\
\hline$\rightarrow$ & & 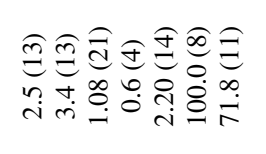 & 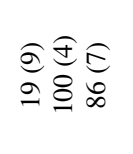 & 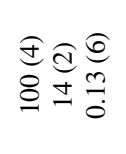 & 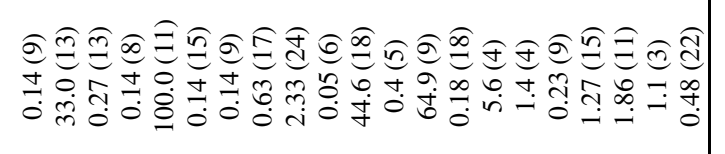 & 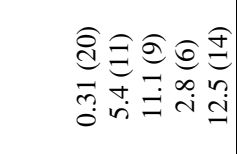 \\
\hline 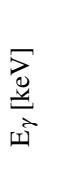 & & 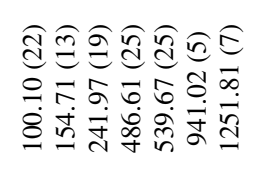 & 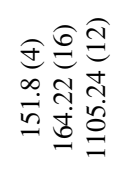 & 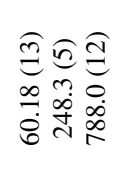 & 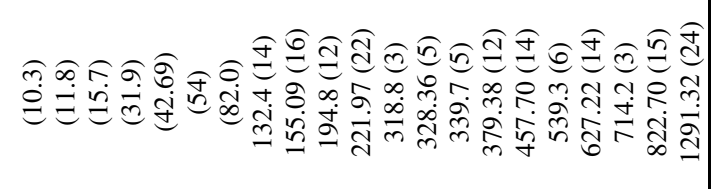 & 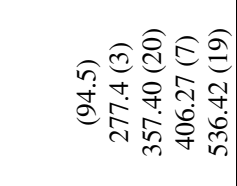 \\
\hline$\widetilde{b}$ & & 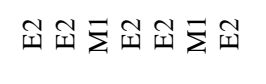 & 외피 피 & $\overline{\text { 포 }} \bar{\Sigma}$ & 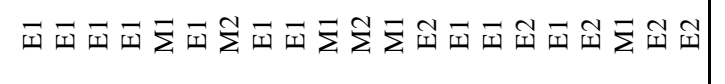 & $\bar{\Sigma} \widetilde{I} \bar{\Sigma} \bar{\Sigma}$ \\
\hline$\underset{\Sigma}{E}$ & & 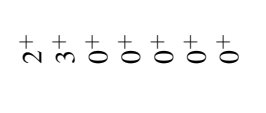 & $+\begin{array}{l}+t \\
+\end{array}$ & 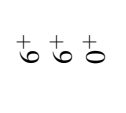 & 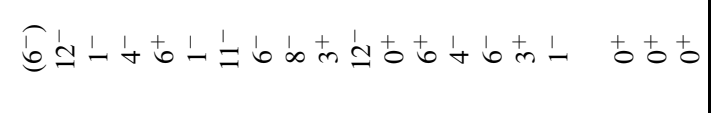 & 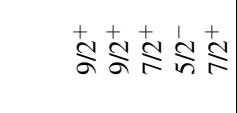 \\
\hline $\begin{array}{l}E \\
E\end{array}$ & & 守庄古古市古古 & $+t_{0}^{+}$ & $\pm+\infty+\infty$ & 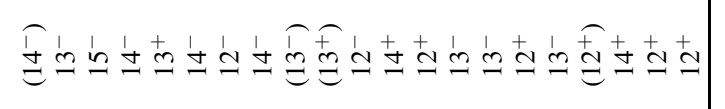 & 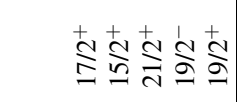 \\
\hline $\begin{array}{ll}E \\
\Sigma\end{array}$ & & to & 6 & $\infty$ & $\stackrel{+}{ \pm}$ & $\frac{9}{2}$ \\
\hline 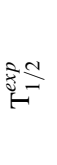 & & 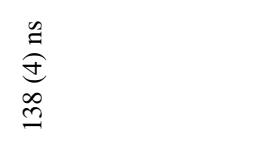 & 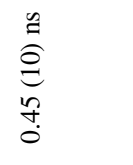 & 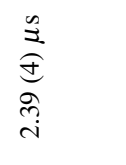 & 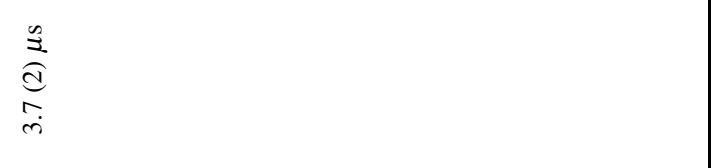 & $\underset{\substack{\xi \\
ٍ}}{\stackrel{\infty}{\infty}}$ \\
\hline iv & 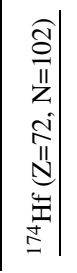 & 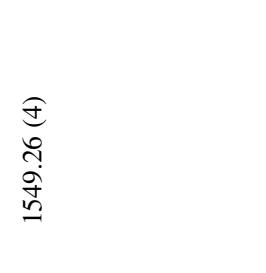 & $\begin{array}{l}\hat{E} \\
\stackrel{q}{q} \\
\stackrel{g}{E} \\
\underline{E}\end{array}$ & 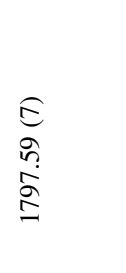 & 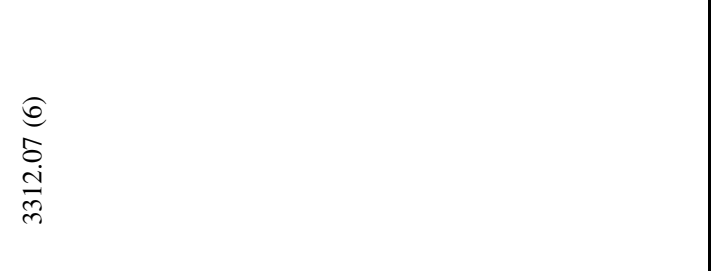 & 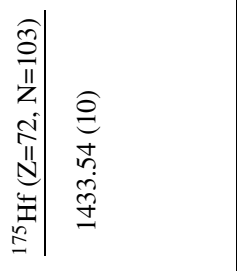 \\
\hline
\end{tabular}




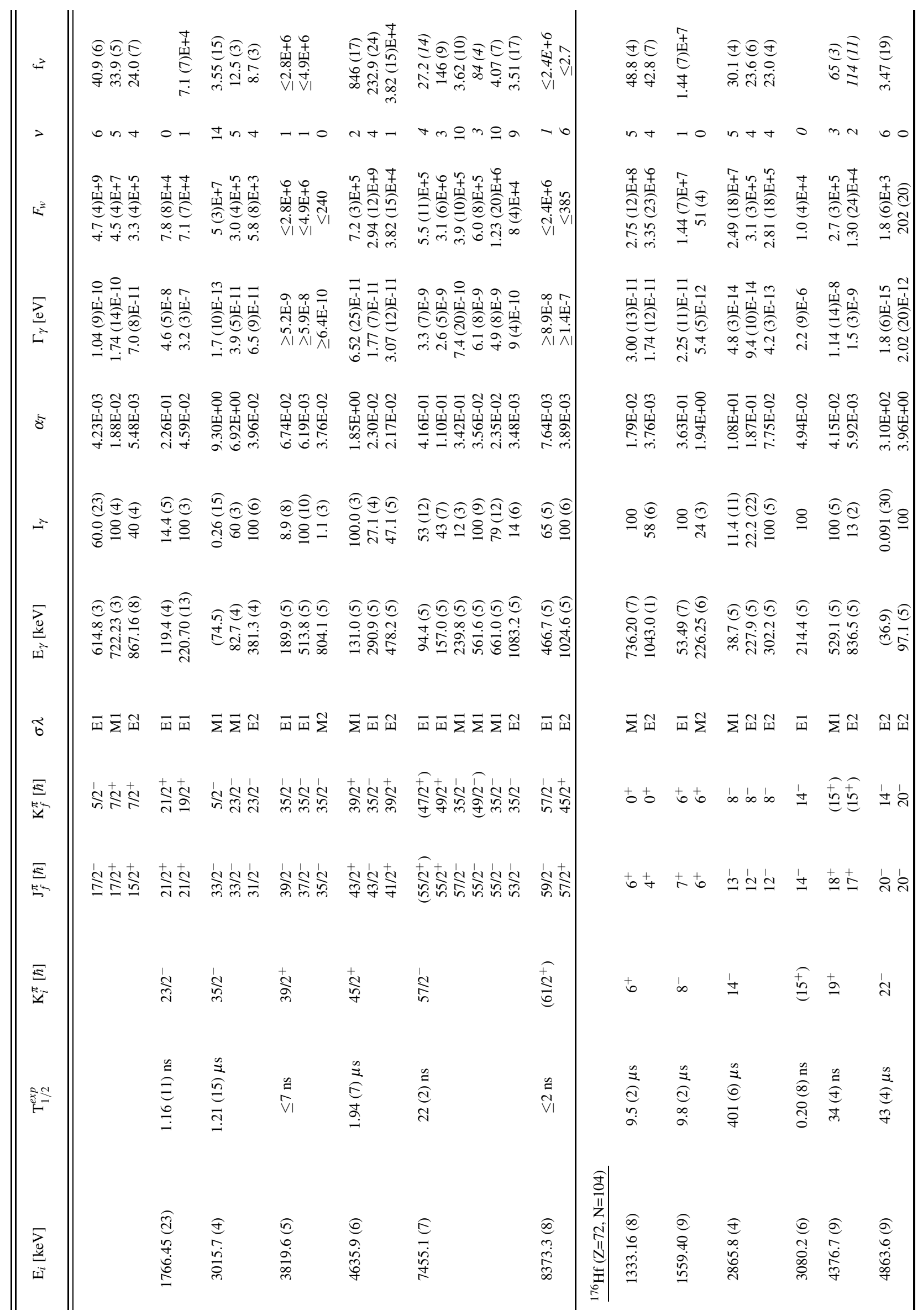




\begin{tabular}{|c|c|c|c|c|c|c|c|c|c|c|c|c|c|c|}
\hline 4 & & 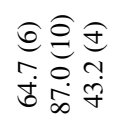 & 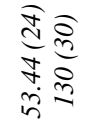 & & 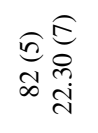 & & & 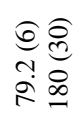 & 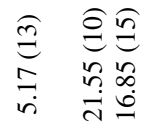 & 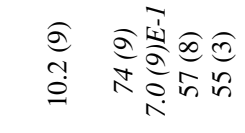 & 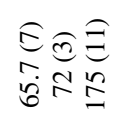 & 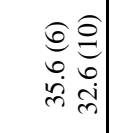 & & 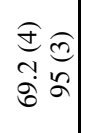 \\
\hline$>$ & & orn & $n \gamma$ & 00 & $m+$ & 0 & & -6 & NOW & omonNnt & $n+m$ & $0 n+$ & & 6 in \\
\hline$x^{3}$ & & 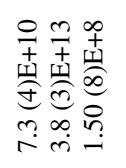 & 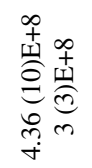 & 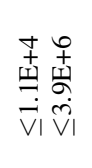 & 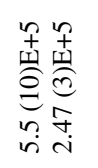 & 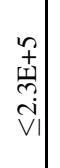 & & 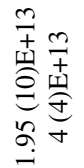 & 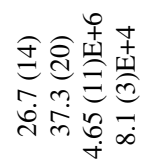 & 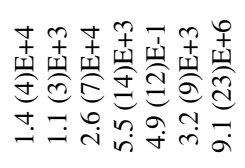 & 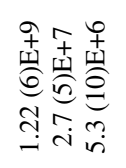 & 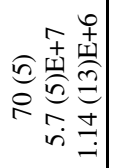 & & 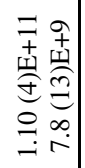 \\
\hline$\frac{\sum_{0}}{2}$ & & 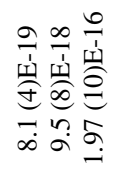 & 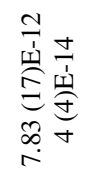 & 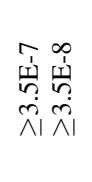 & 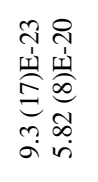 & 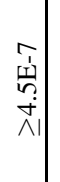 & & 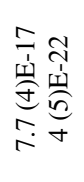 & 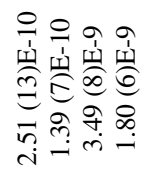 & 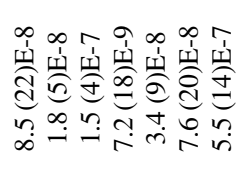 & 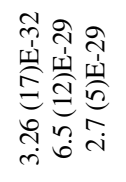 & 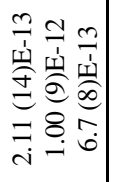 & & 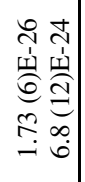 \\
\hline 5 & & 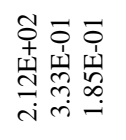 & 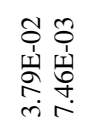 & 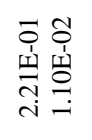 & 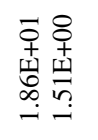 & 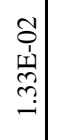 & & 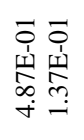 & 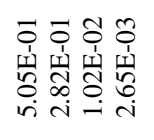 & 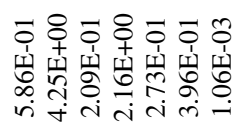 & 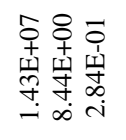 & 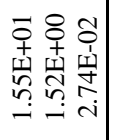 & & 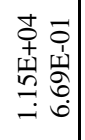 \\
\hline$\Rightarrow$ & & 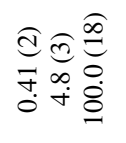 & $8 \stackrel{0}{n}$ & $\begin{array}{l}\Xi 0 \\
\stackrel{0}{0}\end{array}$ & $\begin{array}{l}\hat{\hat{n}} \widehat{\hat{n}} \\
0 \\
0 \\
0 \\
0 \\
0\end{array}$ & 8 & & 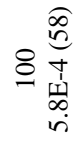 & 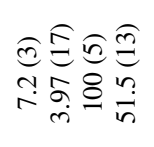 & 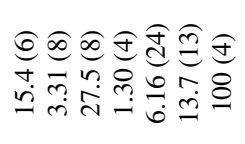 & 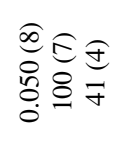 & 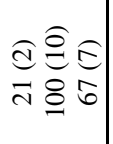 & & 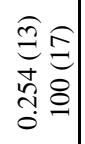 \\
\hline 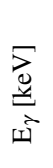 & & 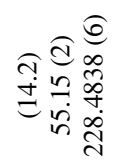 & $\stackrel{0}{\infty}$ & 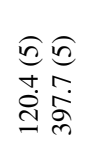 & 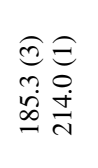 & $\begin{array}{l}n \\
\dot{2} \\
\dot{n}\end{array}$ & & 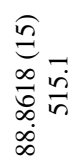 & 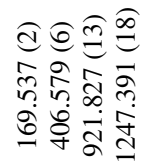 & 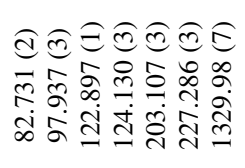 & 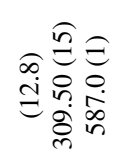 & 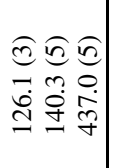 & & 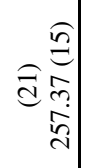 \\
\hline$\widehat{b}$ & & $\bar{\Sigma}$ 可 & $\bar{\Sigma} \tilde{I}$ & 포 $\bar{x}$ & $\sum \pi$ & 피 & & $\bar{\varpi} \Sigma$ & 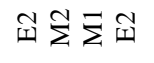 & 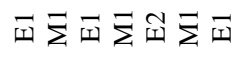 & $m \sum$ & $\tilde{\Sigma} \Sigma \tilde{\Sigma}$ & & $\Sigma \tilde{2}$ \\
\hline $\begin{array}{l}E \\
E \\
E\end{array}$ & & 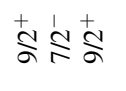 & $\underset{1}{\stackrel{n}{N}}$ & & 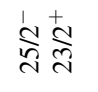 & $\underset{m}{\stackrel{1}{N}}$ & & t。 & $\stackrel{+}{\sim} \infty \begin{array}{l}+ \\
0\end{array}$ & 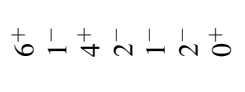 & $\infty \infty^{1} \infty$ & 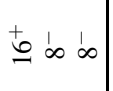 & & 告 \\
\hline $\begin{array}{l}E \\
E \\
E\end{array}$ & & 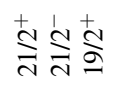 & $\stackrel{1}{\stackrel{1}{N}}$ & 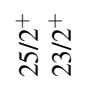 & $\stackrel{\stackrel{+}{S} \stackrel{+}{S}}{\mathrm{~m}}$ & 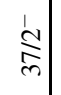 & & $+\infty$ & $+\infty \begin{array}{l}+ \\
+\end{array}$ & 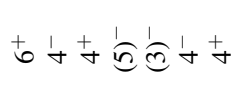 & $\stackrel{1}{ } \simeq$ & $\underset{ \pm}{ \pm} \simeq$ & & $\underset{\mathrm{N}}{\mathrm{N}} \stackrel{2}{9}$ \\
\hline $\begin{array}{l}E \\
5 \\
\Sigma\end{array}$ & & $\stackrel{+}{\stackrel{\lambda}{d}}$ & $\frac{\widehat{T}}{\frac{1}{g}}$ & $\frac{1}{\sqrt{2}}$ & $\underset{m}{\stackrel{1}{c}}$ & సે & & $\infty$ & \pm & in & $\stackrel{+}{ \pm}$ & I & & 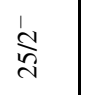 \\
\hline$\stackrel{5}{=}=$ & & 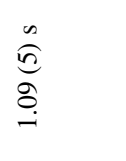 & 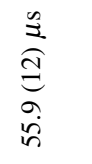 & $\begin{array}{l}\stackrel{a}{g} \\
\vec{V} \mid\end{array}$ & 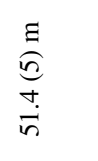 & $\begin{array}{c}0 \\
\vdots \\
\vec{V} \mid\end{array}$ & & $\underset{\substack{d \\
\stackrel{+}{+}}}{\stackrel{n}{+}}$ & 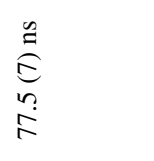 & 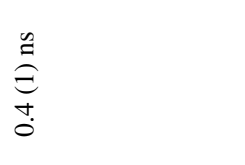 & $\underset{\vec{m}}{\stackrel{\lambda}{\Xi}}$ & 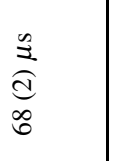 & & 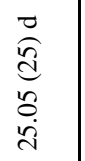 \\
\hline 总 & 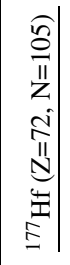 & 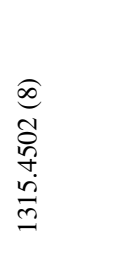 & 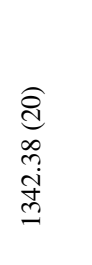 & $\begin{array}{l}\underset{6}{=} \\
\frac{n}{2} \\
\stackrel{m}{I} \\
=\end{array}$ & 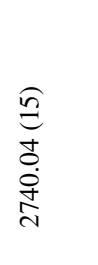 & 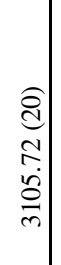 & 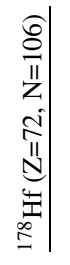 & 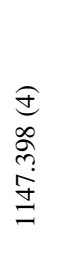 & 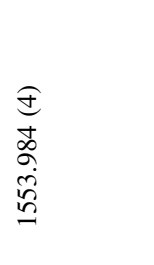 & 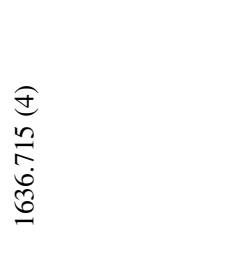 & \begin{tabular}{l}
$\hat{0}$ \\
$o$ \\
0 \\
0 \\
\multirow{+}{*}{} \\
d
\end{tabular} & 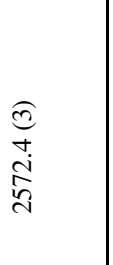 & 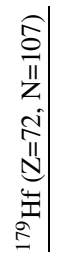 & 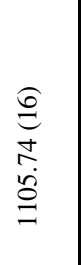 \\
\hline
\end{tabular}




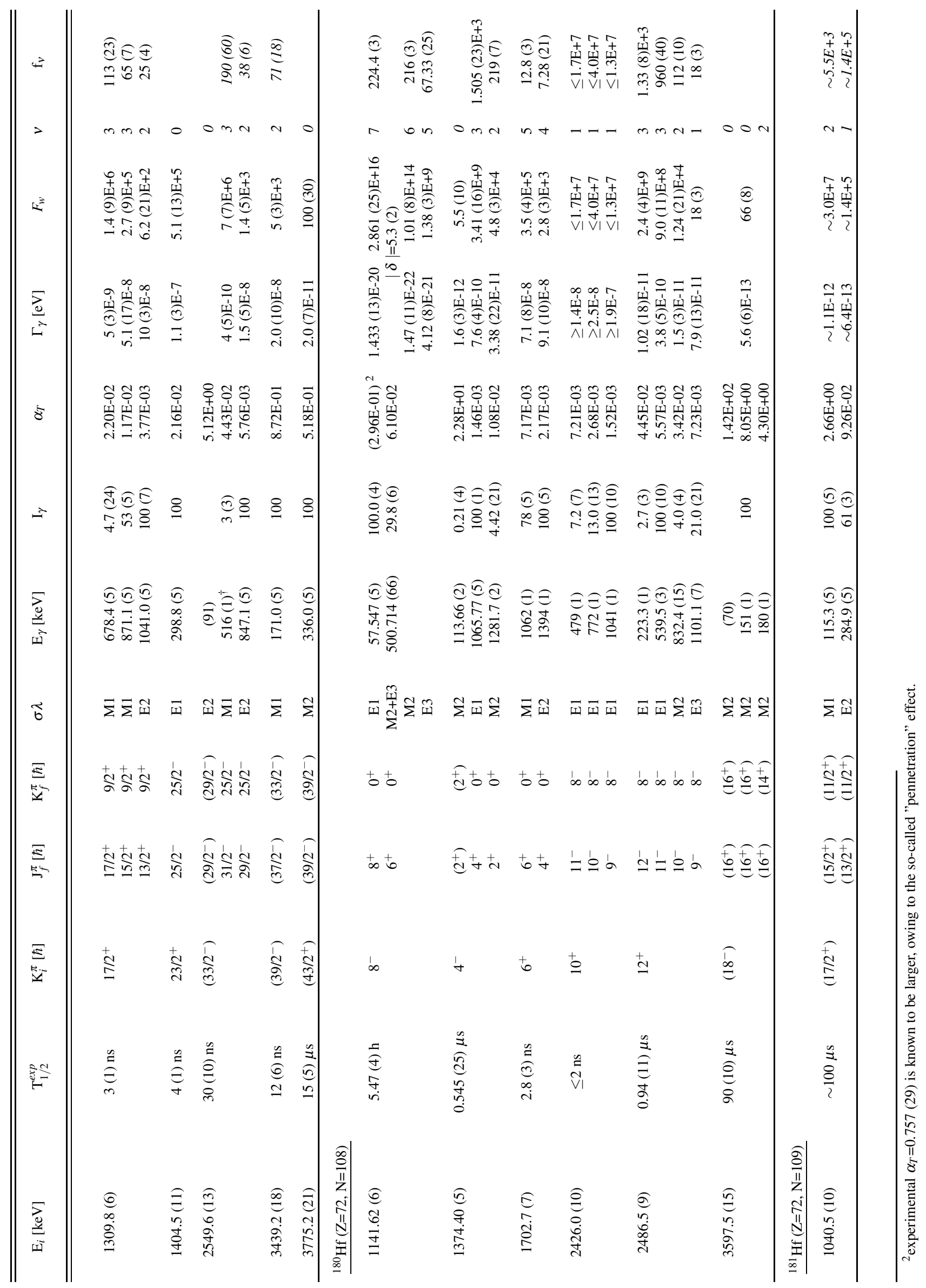




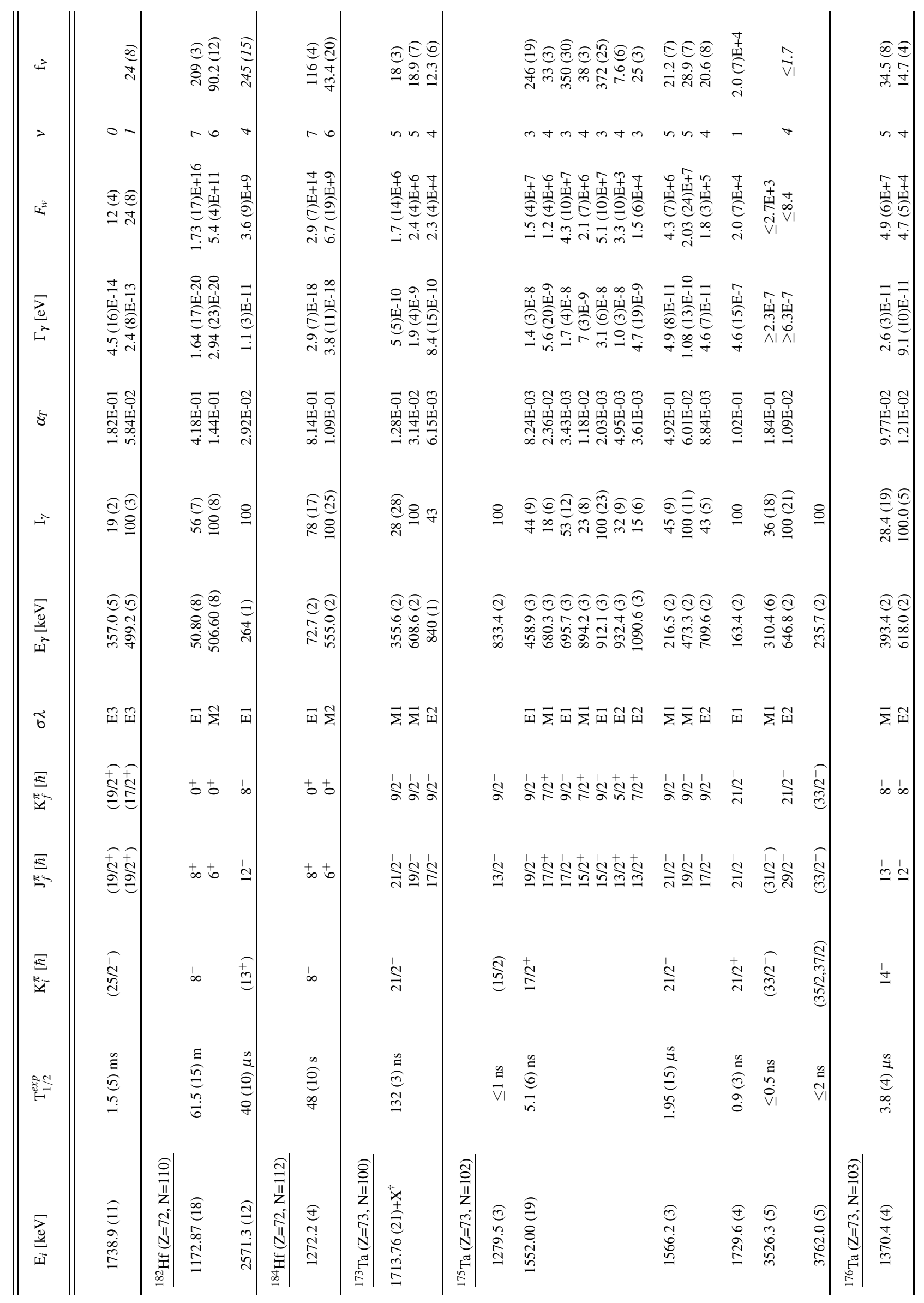




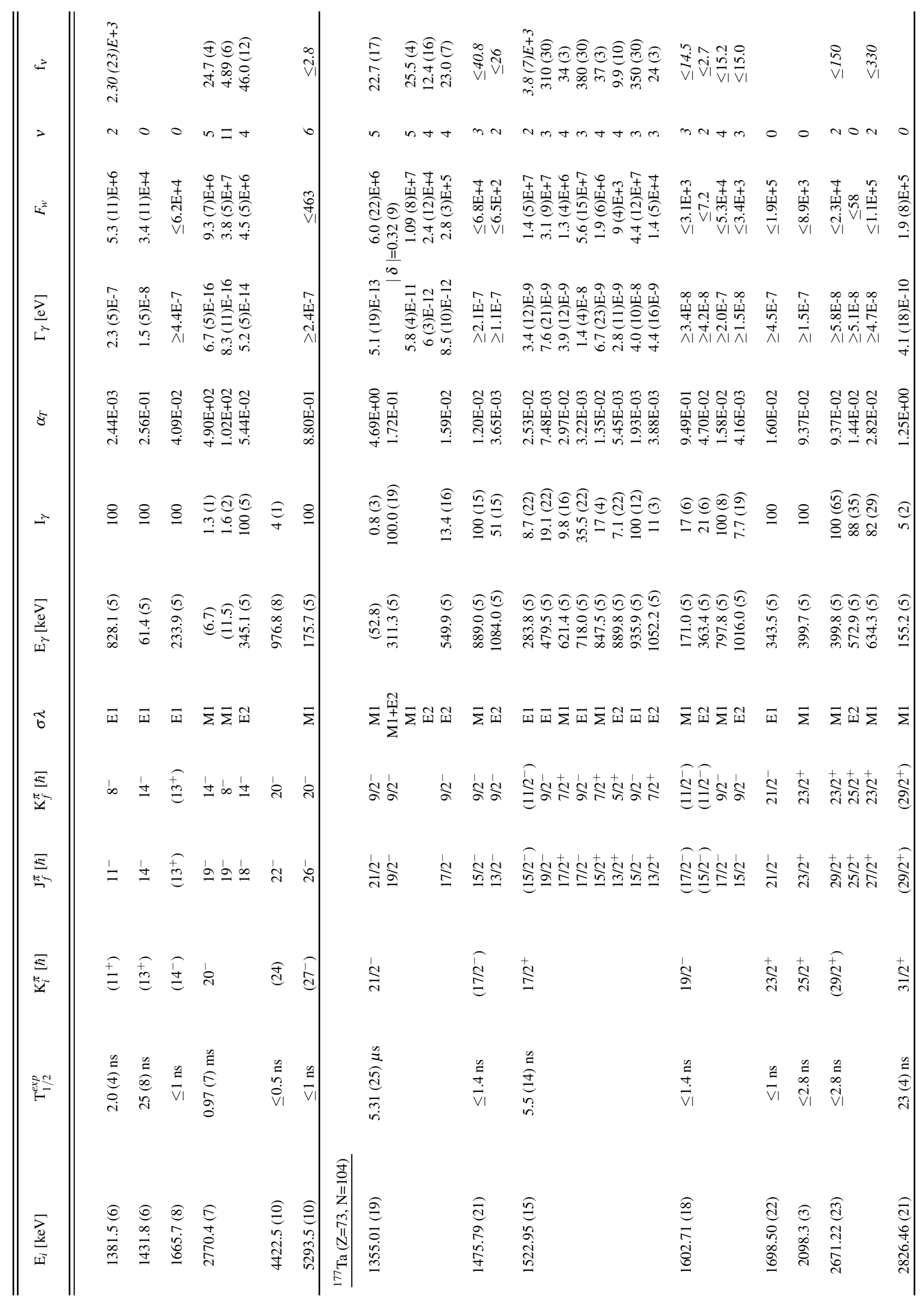




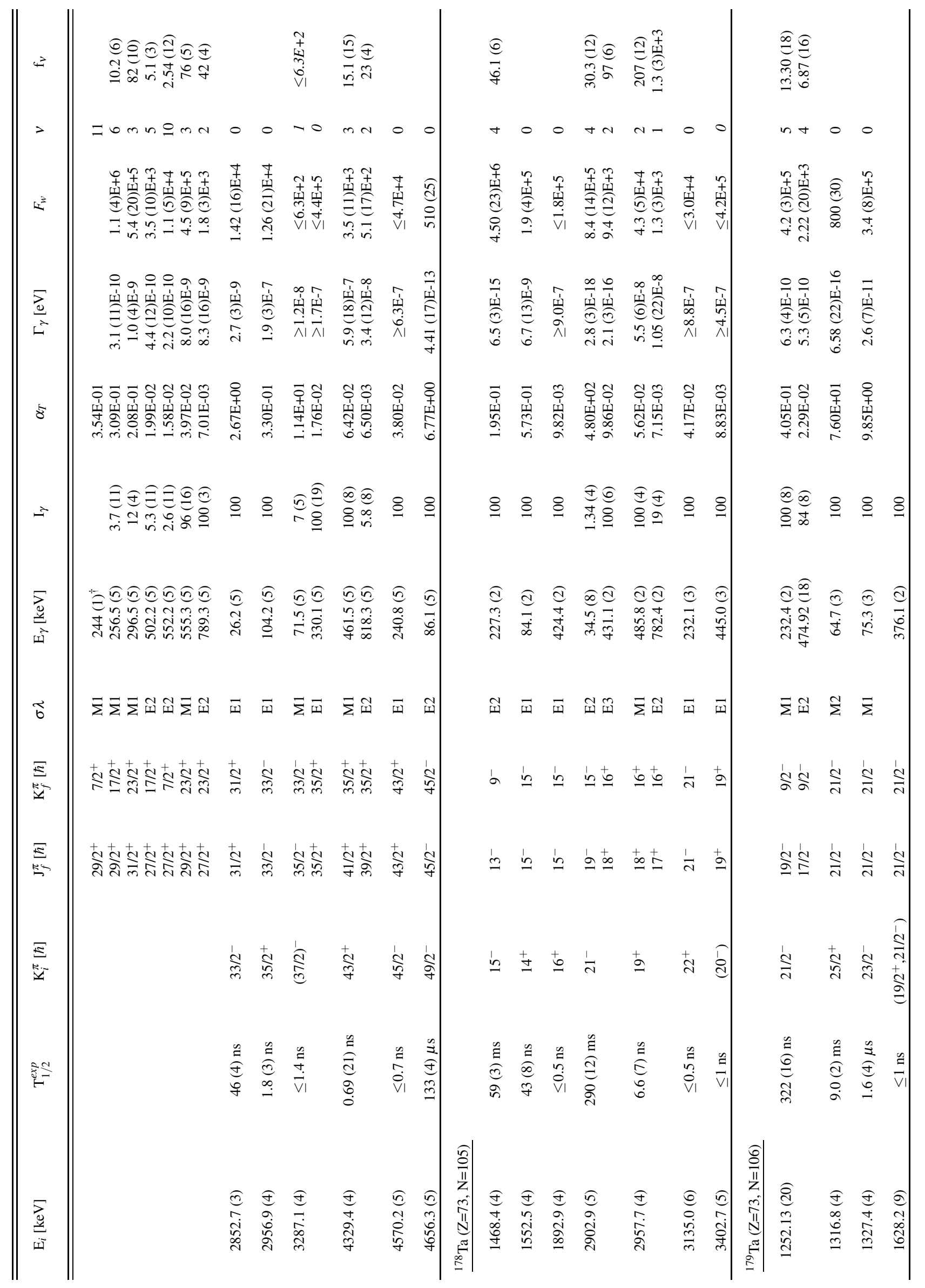




\begin{tabular}{|c|c|c|}
\hline 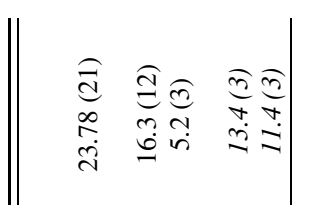 & 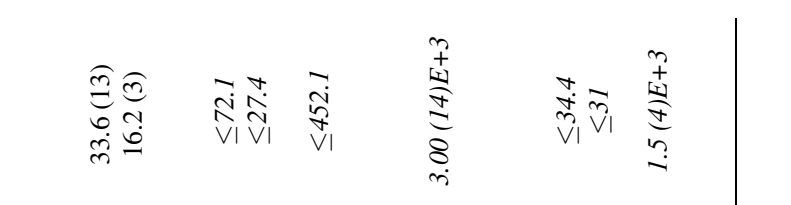 & 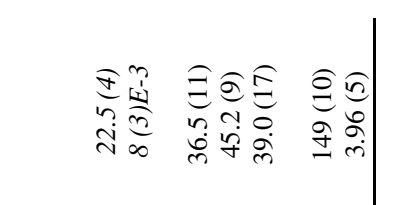 \\
\hline 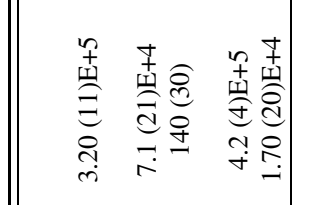 & 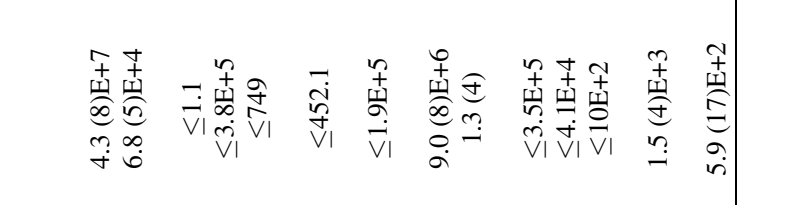 & 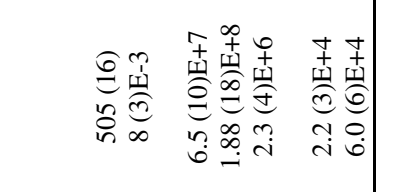 \\
\hline 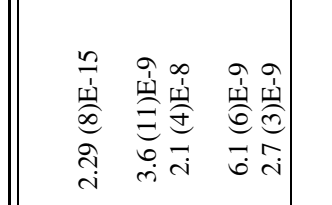 & 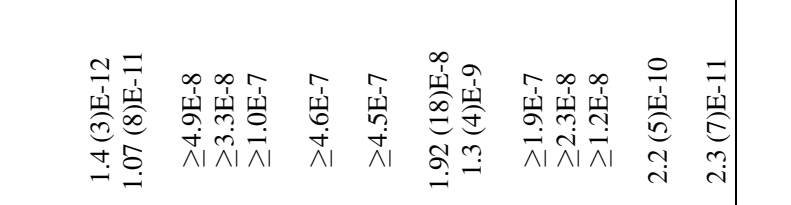 & 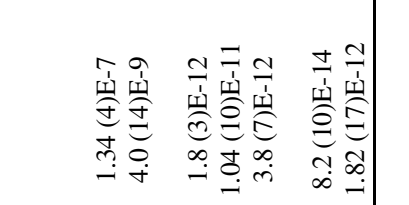 \\
\hline 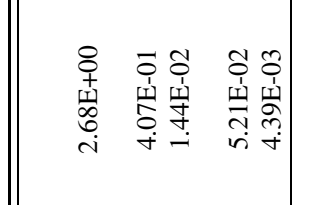 & 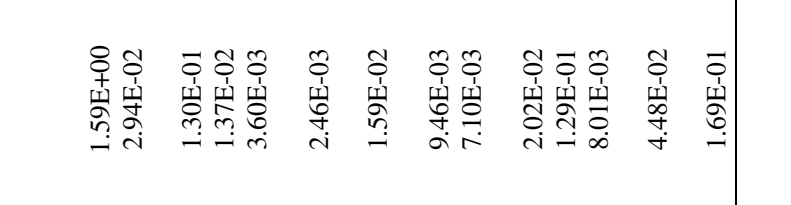 & 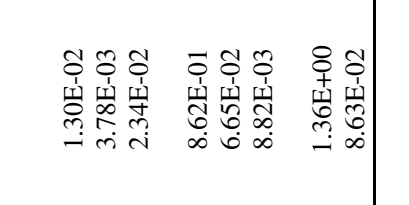 \\
\hline 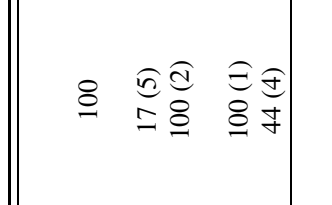 & 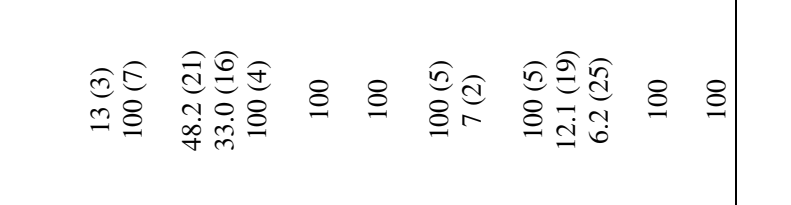 & 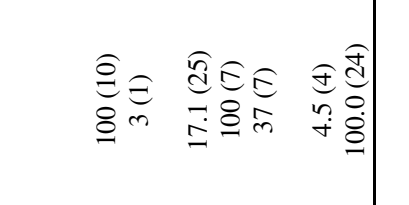 \\
\hline 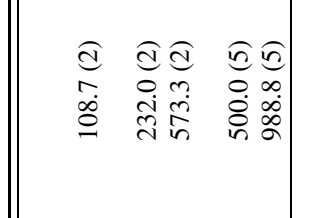 & 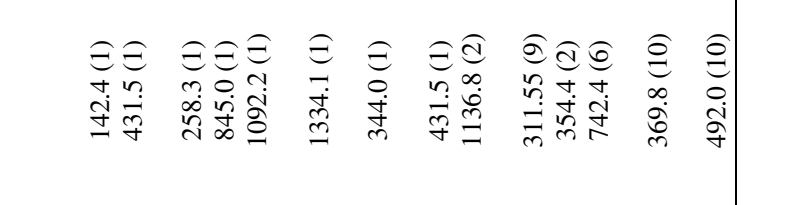 & 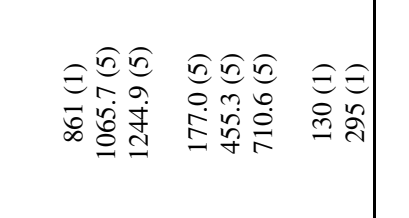 \\
\hline & 部 & \\
\hline & 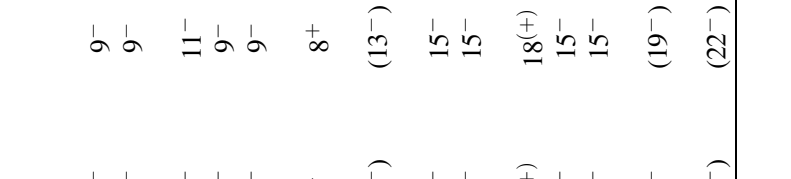 & \\
\hline & 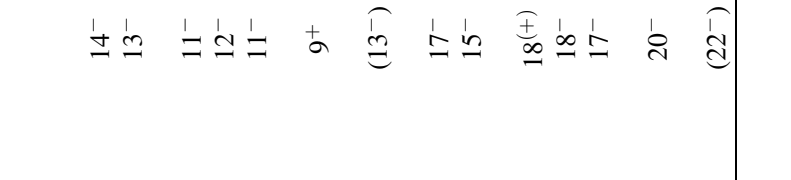 & 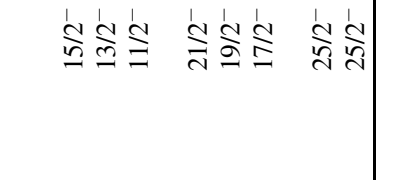 \\
\hline & 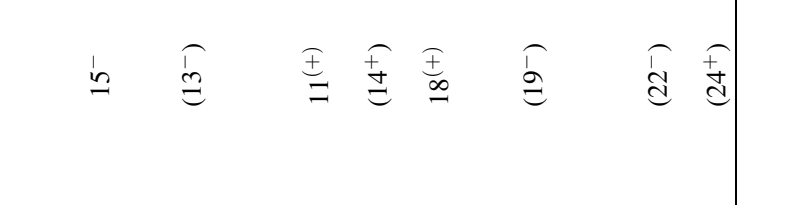 & 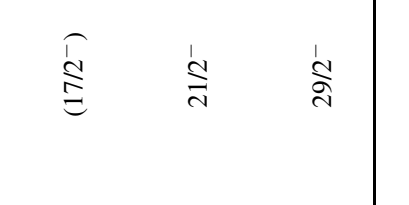 \\
\hline & 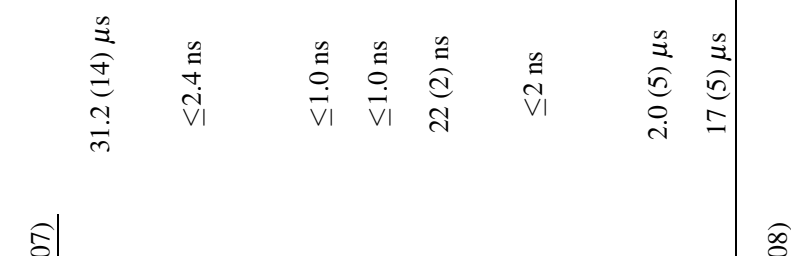 & \\
\hline
\end{tabular}




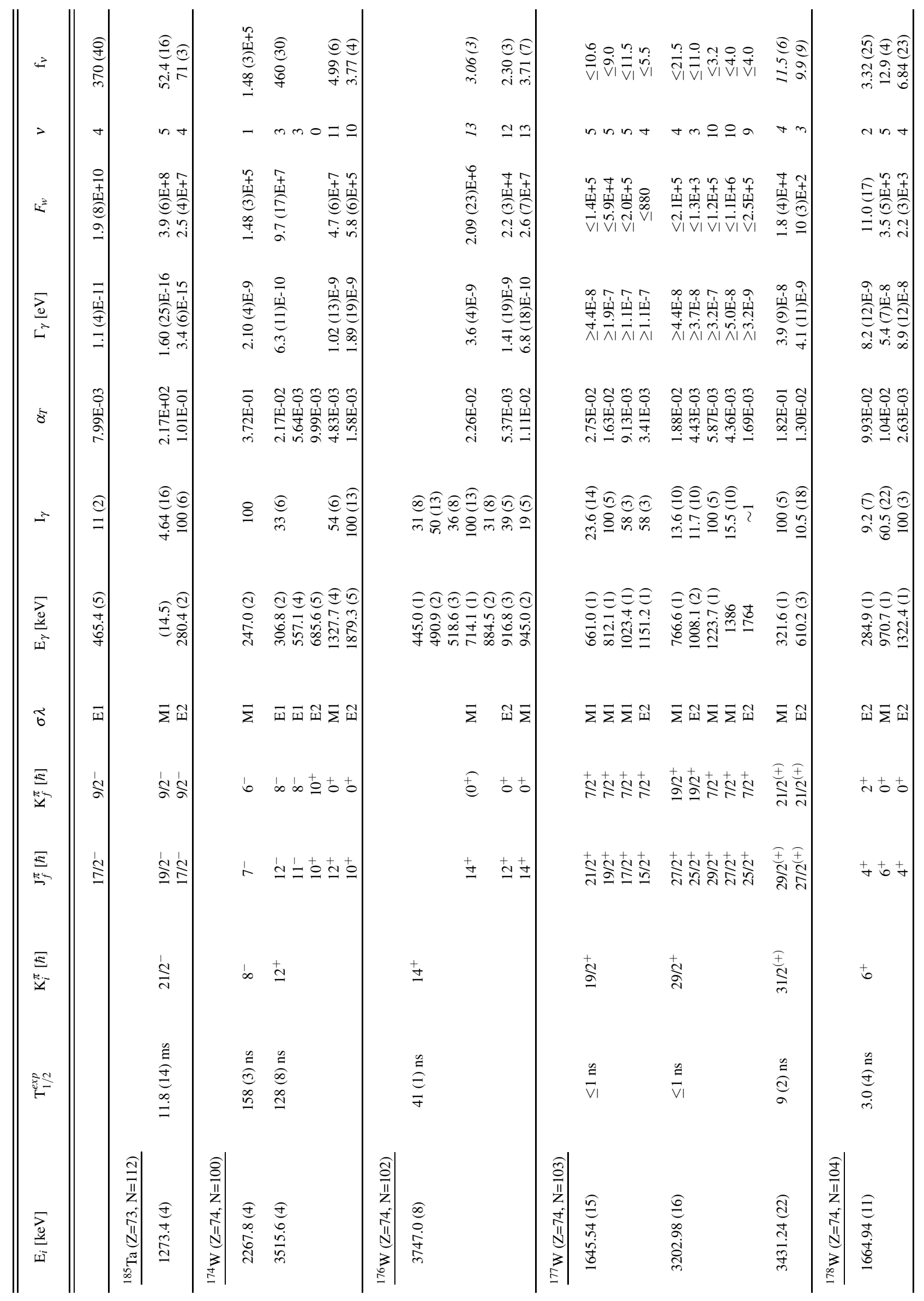




\begin{tabular}{|c|c|c|c|c|c|c|c|c|c|c|c|c|}
\hline$\rightarrow$ & 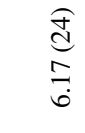 & 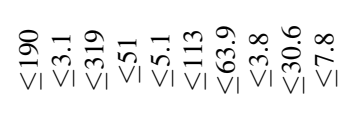 & 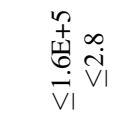 & 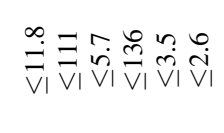 & & & & 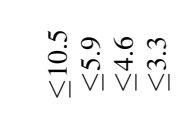 & 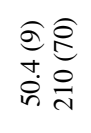 & 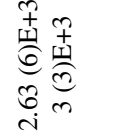 & $\begin{array}{l}\stackrel{m}{+} \\
\stackrel{m}{m} \\
\stackrel{+}{v}\end{array}$ & 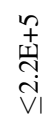 \\
\hline$>$ & om & 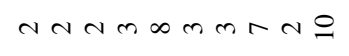 & $0-=$ & $n+\nabla+=0$ & 00 & 0 & 0 & $o+n=a$ & $N-$ & $N-0$ & $N$ & - \\
\hline$x^{3}$ & 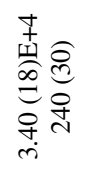 & 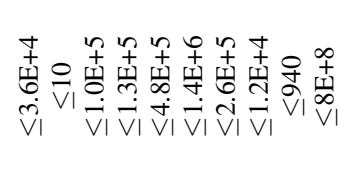 & 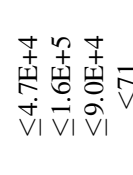 & 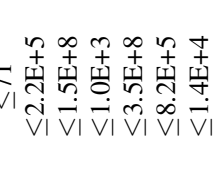 & 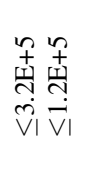 & 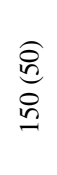 & 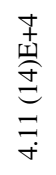 & 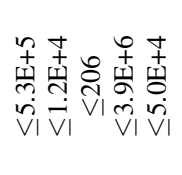 & 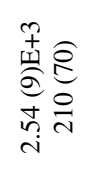 & 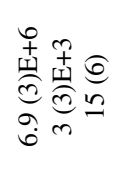 & 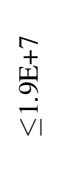 & 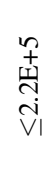 \\
\hline 胥 & 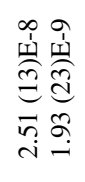 & 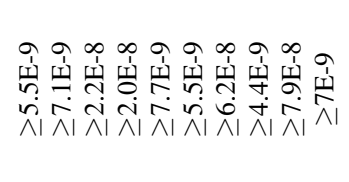 & 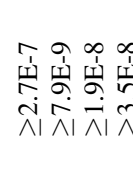 & 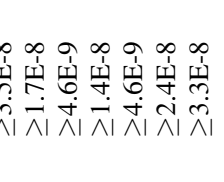 & 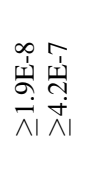 & 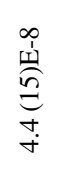 & 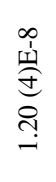 & 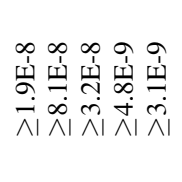 & 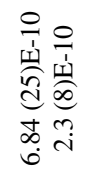 & 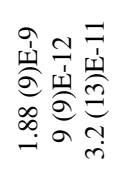 & 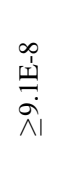 & \\
\hline$\S$ & 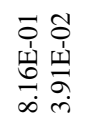 & 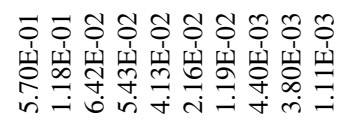 & 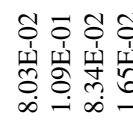 & 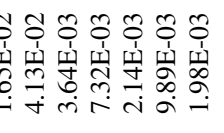 & 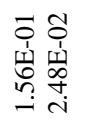 & 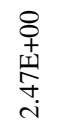 & 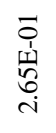 & 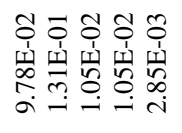 & 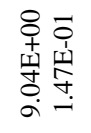 & 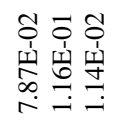 & 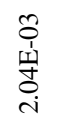 & 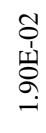 \\
\hline$\Rightarrow$ & 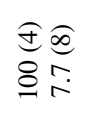 & 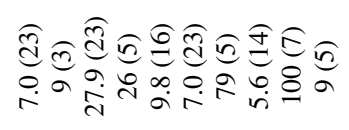 & 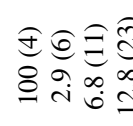 & 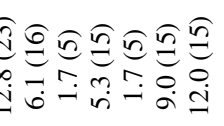 & $\begin{array}{l}\hat{n} \underset{0}{0} \\
+ \\
+ \\
+\end{array}$ & 8 & 8 & 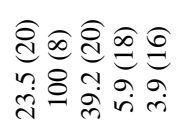 & 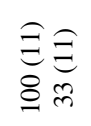 & 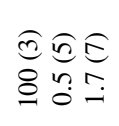 & $\stackrel{8}{\circ}$ & $\stackrel{6}{\circ}$ \\
\hline 鹰 & 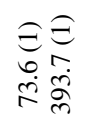 & 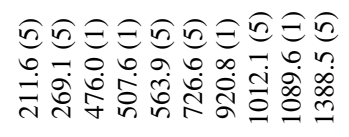 & 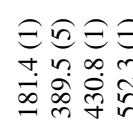 & 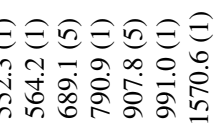 & 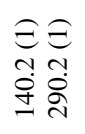 & $\underset{\substack{\Xi \\
\infty \\
\infty \\
\infty}}{ }$ & 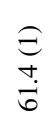 & 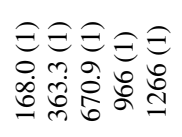 & 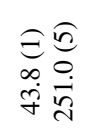 & 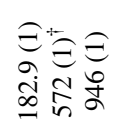 & $\begin{array}{l}\hat{\Xi} \\
\text { oे } \\
\text { on }\end{array}$ & 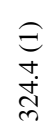 \\
\hline$\widetilde{b}$ & 푀 포 & 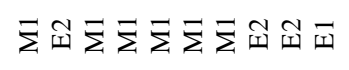 & $\bar{\Psi} \bar{\Sigma} \bar{\Sigma} \tilde{I}$ & 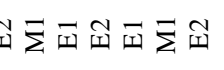 & 포피 & $\bar{\Sigma}$ & 포 & 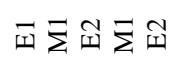 & $\Sigma \widetilde{I}$ & 牙芒色 & 피 & 피 \\
\hline $\begin{array}{l}E \\
E\end{array}$ & to & 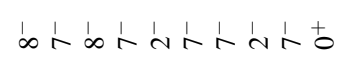 & $\stackrel{+}{さ}$ & むんさんする。 & $\stackrel{+}{\simeq} さ$ & $\stackrel{m}{m}$ & \pm & 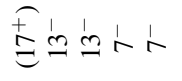 & $\stackrel{1}{\infty} \stackrel{1}{\infty}$ & ปั่ & $\stackrel{+}{a}$ & $\stackrel{\infty}{\sim}$ \\
\hline$E$ & to in & 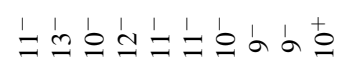 & 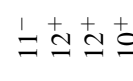 & 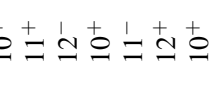 & $\stackrel{+}{m} \pm$ & $\stackrel{m}{m}$ & \pm & 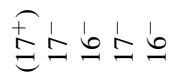 & $\grave{n}$ & $\operatorname{dyn}$ & $\stackrel{+}{\star}$ & ते \\
\hline $\begin{array}{l}E \\
E \\
E\end{array}$ & $r$ & $=$ & $\stackrel{+}{\simeq}$ & & $\stackrel{m}{m}$ & $\stackrel{1}{ \pm}$ & $\stackrel{+}{2}$ & $\stackrel{\infty}{\infty}$ & $\frac{1}{4}$ & $\stackrel{+}{a}$ & 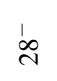 & $\stackrel{+}{\circ}$ \\
\hline$\stackrel{5}{3}=$ & 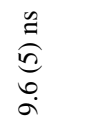 & $\begin{array}{l}n \\
\stackrel{n}{N} \\
V 1\end{array}$ & $\stackrel{\mathscr{a}}{\vec{\nabla} \mid}$ & & $\stackrel{n}{\vec{\nabla} \mid}$ & 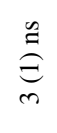 & $\stackrel{\varrho}{\Xi}$ & $\begin{array}{c}n \\
\stackrel{n}{V}\end{array}$ & $\begin{array}{l}a \\
\stackrel{a}{d} \\
\text { to }\end{array}$ & & $\begin{array}{c}n \\
\stackrel{n}{n} \\
v_{1}\end{array}$ & $\stackrel{\varrho}{\Xi}$ \\
\hline 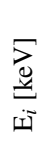 & 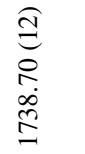 & 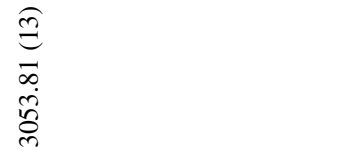 & 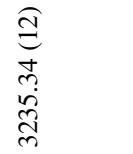 & & \begin{tabular}{l}
$n$ \\
\multirow{n}{n}{} \\
$n$ \\
$n$ \\
$n$ \\
$n$ \\
$n$
\end{tabular} & 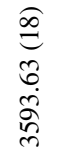 & 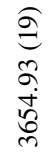 & 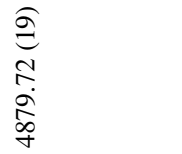 & $\begin{array}{l}\hat{n} \\
\stackrel{n}{m} \\
\stackrel{n}{n}\end{array}$ & 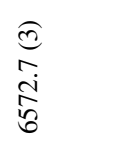 & $\begin{array}{l}\underset{+}{J} \\
+ \\
\infty \\
\stackrel{\infty}{+} \\
\infty\end{array}$ & $\begin{array}{l}\mathcal{\Xi} \\
m \\
8 \\
\infty \\
\infty\end{array}$ \\
\hline
\end{tabular}




\begin{tabular}{|c|c|c|c|c|c|c|c|c|c|c|c|c|c|c|c|}
\hline$\Rightarrow$ & 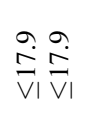 & & $\stackrel{\partial}{\vec{\nabla} \mid}$ & & 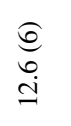 & 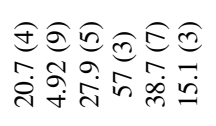 & & 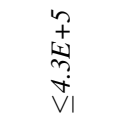 & & 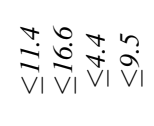 & 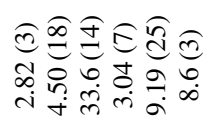 & & & & $\stackrel{\widehat{\overbrace{}}}{\stackrel{\infty}{\infty}}$ \\
\hline$>$ & $\nabla m$ & 0 & $n \sim$ & & $\bullet$ & nnn $n$ ont & $\circ$ & $0-0$ & 0 & $n+N m$ & $\mathscr{9} \operatorname{In} \simeq \simeq$ & 0 & 00 & 00 & NO \\
\hline$x^{3}$ & 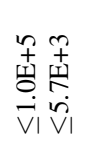 & 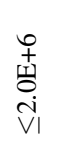 & 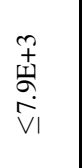 & & 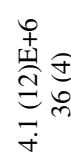 & 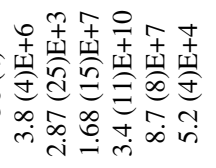 & 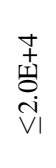 & 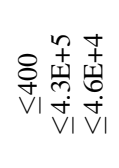 & 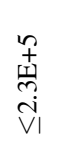 & 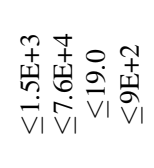 & 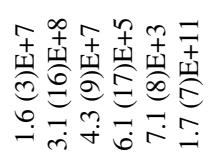 & $\begin{array}{l}\underset{+}{ \pm} \\
\stackrel{\oplus}{\oplus} \\
\overrightarrow{\tilde{v}}\end{array}$ & 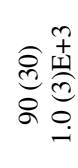 & 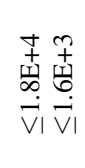 & 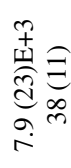 \\
\hline 雍 & 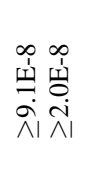 & $\begin{array}{l}\stackrel{\hat{n}}{4} \\
\stackrel{n}{\wedge} \\
\stackrel{+}{\wedge}\end{array}$ & 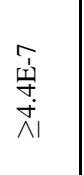 & & 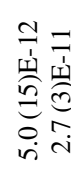 & 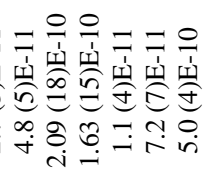 & 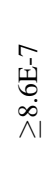 & 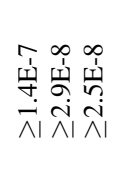 & 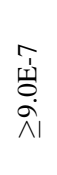 & 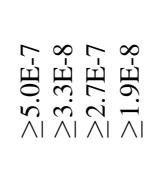 & 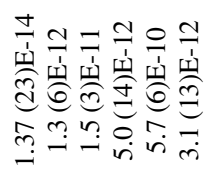 & 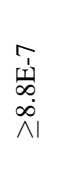 & 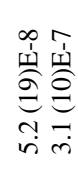 & 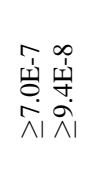 & 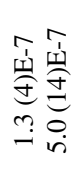 \\
\hline 8 & 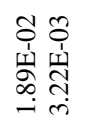 & 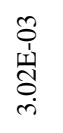 & 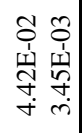 & & 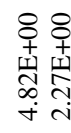 & 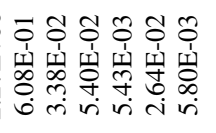 & 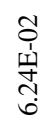 & 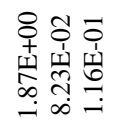 & 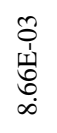 & 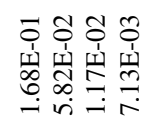 & 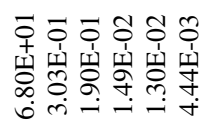 & 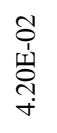 & 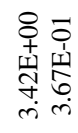 & 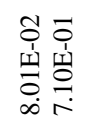 & 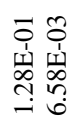 \\
\hline$\approx$ & 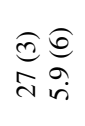 & $\stackrel{8}{\circ}$ & 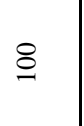 & & 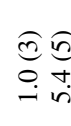 & 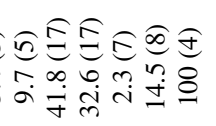 & 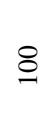 & 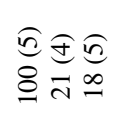 & 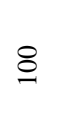 & 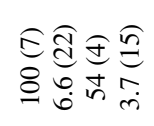 & 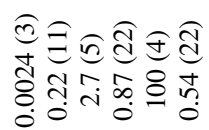 & $\nsubseteq$ & $\begin{array}{l}6 \sqrt{0} \\
\simeq 气\end{array}$ & 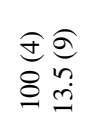 & 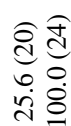 \\
\hline 恿 & 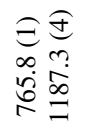 & 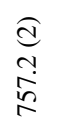 & 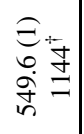 & & 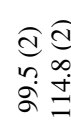 & 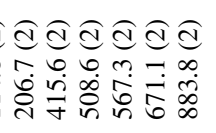 & 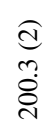 & 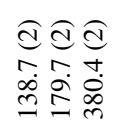 & 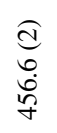 & 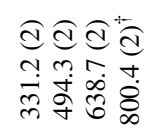 & 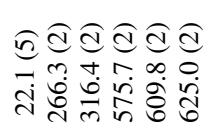 & 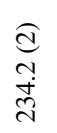 & $\begin{array}{l}\widehat{d} \widehat{d} \\
0 \\
0 \\
\vec{b} \\
\stackrel{d}{d}\end{array}$ & 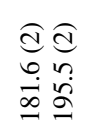 & 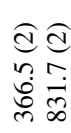 \\
\hline$\vec{b}$ & $\sum \tilde{y}$ & $\vec{r}$ & $\sum \widetilde{\Sigma}$ & & $\Sigma \tilde{\Sigma}$ & 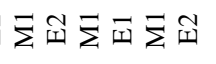 & $\overrightarrow{\text { 피 }}$ & $\bar{\Sigma} \bar{I} \bar{\Sigma}$ & $\bar{ㅍ}$ & 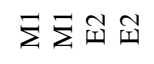 & 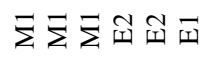 & 피 & $\bar{\Sigma} \bar{\Sigma}$ & 可主 & $\bar{\Sigma} \tilde{I}$ \\
\hline$\underset{E}{E}$ & 点点 & $\stackrel{\infty}{i}$ & $\begin{array}{lll}+ & + \\
0 & 0 \\
0\end{array}$ & & & 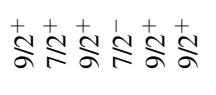 & $\stackrel{+}{\stackrel{n}{s}}$ & 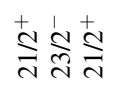 & $\stackrel{+}{\stackrel{N}{N}}$ & 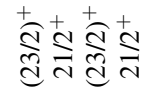 & 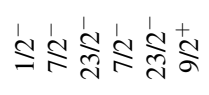 & $\frac{1}{\sqrt[m]{n}}$ & 颃 & $\underset{m}{\stackrel{1}{c} \underset{m}{N}}$ & $\stackrel{+}{\stackrel{+}{S}}$ \\
\hline$\underset{s}{E}$ & $\stackrel{+}{a}+\infty$ & $\stackrel{\infty}{\sim}$ & $\stackrel{+}{m} \stackrel{ \pm}{m}$ & & 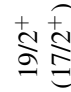 & 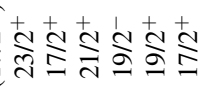 & $\stackrel{+}{\stackrel{N}{N}}$ & 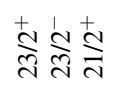 & $\stackrel{+}{\stackrel{n}{c}}$ & 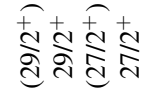 & 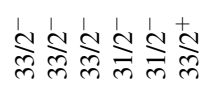 & $\frac{1}{\sqrt{n}}$ & 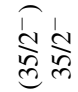 & 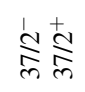 & 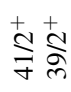 \\
\hline$\Xi$ & & ๙ิ & 装 & & $\stackrel{+}{\stackrel{N}{N}}$ & & 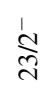 & $\underset{\substack{\text { d } \\
\text { d }}}{+}$ & 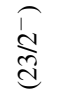 & 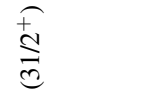 & $\frac{1}{N}$ & $\underset{m}{\stackrel{+}{N}}$ & $\underset{m}{\stackrel{1}{2}}$ & $\stackrel{+}{\stackrel{1}{c}}$ & $\stackrel{+}{\stackrel{y}{\&}}$ \\
\hline 至 & & $\begin{array}{l}\mathscr{a} \\
\vec{V} \mid\end{array}$ & $\stackrel{\mathscr{a}}{\vec{V} \mid}$ & & 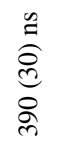 & & $\begin{array}{l}n \\
\vdots \\
n \\
\dot{V}\end{array}$ & 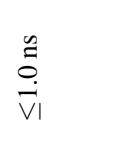 & $\begin{array}{l}n \\
\tilde{n} \\
\tilde{b} \\
\vec{V} \mid\end{array}$ & 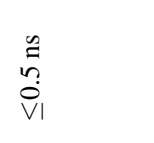 & $\begin{array}{l}\frac{n}{a} \\
\stackrel{a}{0} \\
\stackrel{0}{n}\end{array}$ & $\begin{array}{l}n \\
\stackrel{2}{n} \\
\tilde{v} \\
\vec{v}\end{array}$ & $\begin{array}{l}\stackrel{a}{a} \\
\stackrel{d}{0}\end{array}$ & $\begin{array}{l}n \\
ٌ \\
n \\
\dot{V}\end{array}$ & $\begin{array}{l}\stackrel{n}{a} \\
\stackrel{0}{0}\end{array}$ \\
\hline 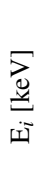 & & $\begin{array}{l}\hat{f} \\
\stackrel{0}{0} \\
\stackrel{0}{0} \\
\infty\end{array}$ & $\begin{array}{l}n \\
n \\
n \\
0 \\
0 \\
=\end{array}$ & 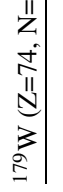 & 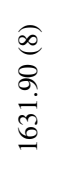 & & 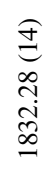 & 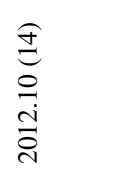 & 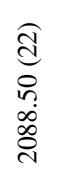 & 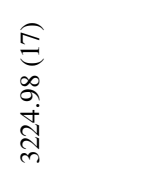 & 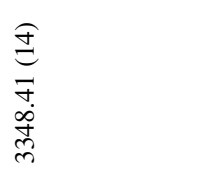 & 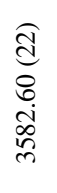 & 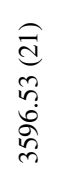 & 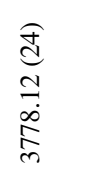 & $\begin{array}{l}\widehat{0} \\
0 \\
0 \\
0 \\
0 \\
0\end{array}$ \\
\hline
\end{tabular}




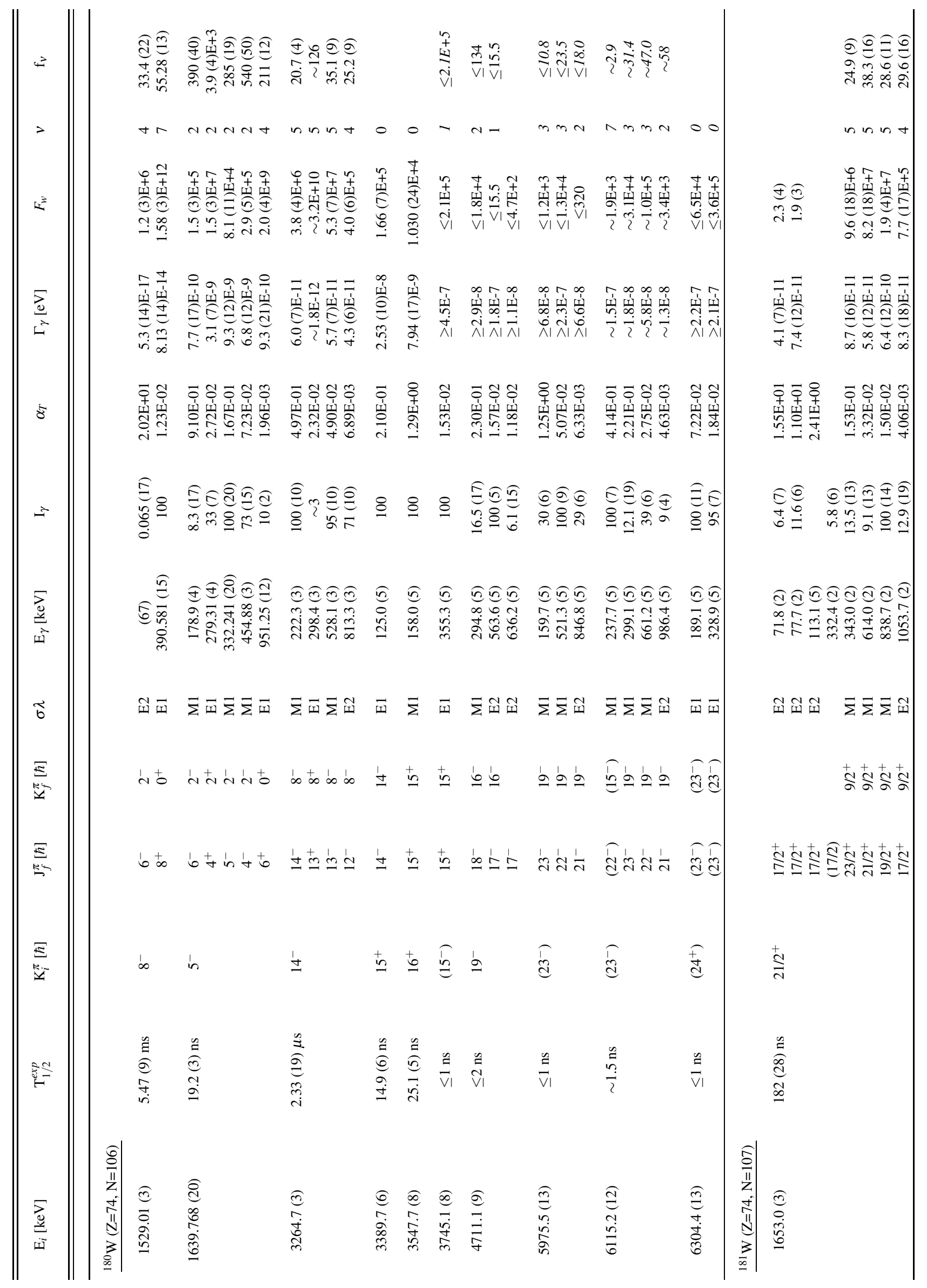




\begin{tabular}{|c|c|c|}
\hline$\frac{\frac{\partial}{x}}{\bar{z}}$ & 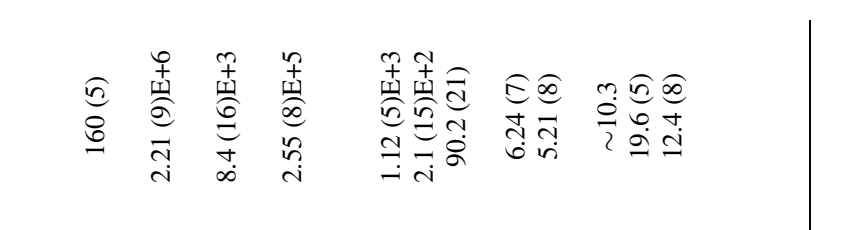 & 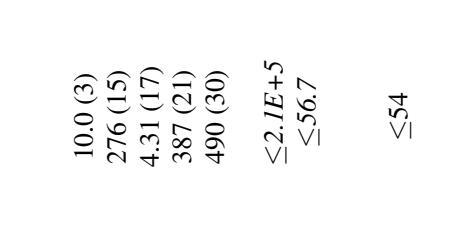 \\
\hline & 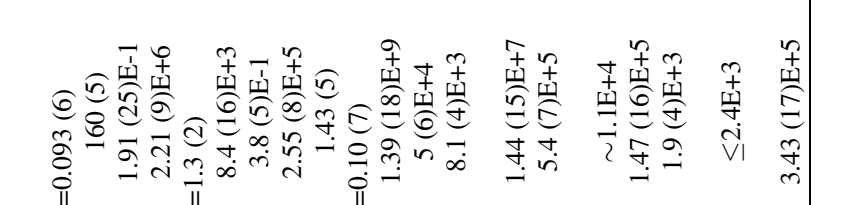 & 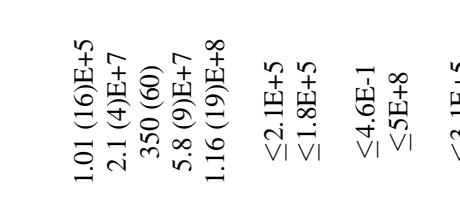 \\
\hline 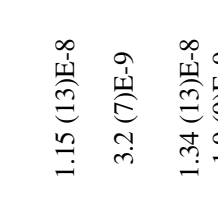 & 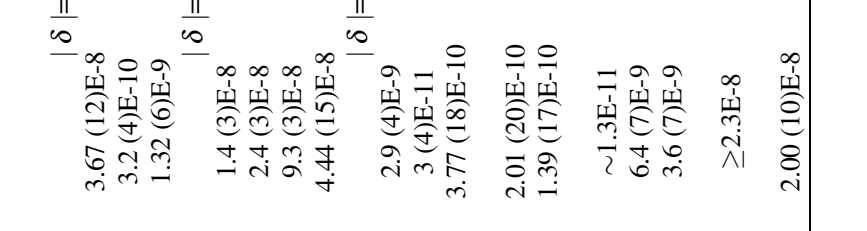 & 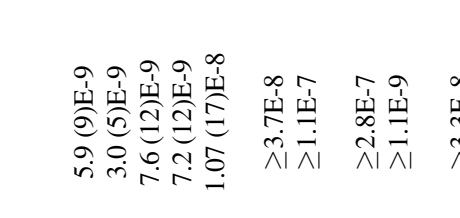 \\
\hline 高 & 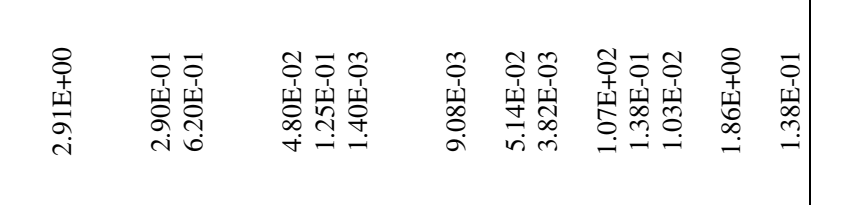 & 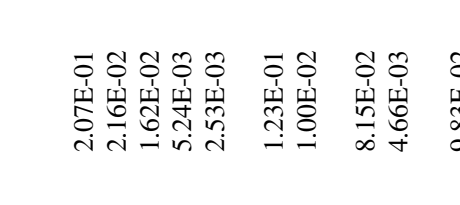 \\
\hline 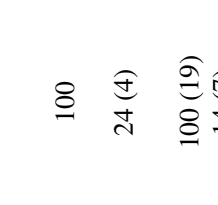 & 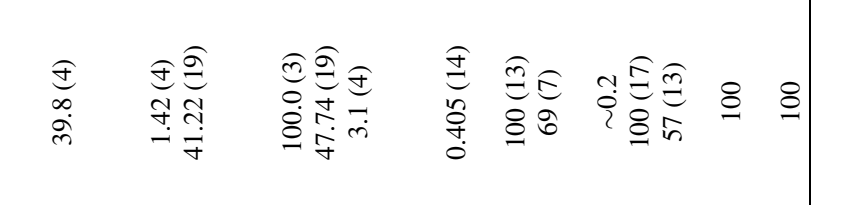 & 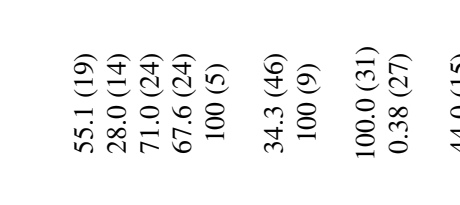 \\
\hline 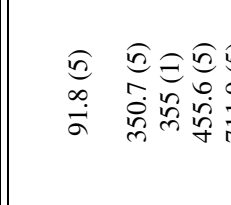 & 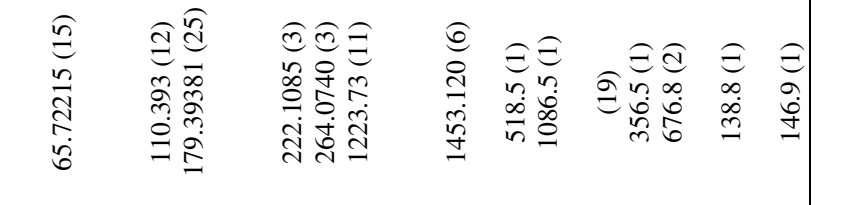 & 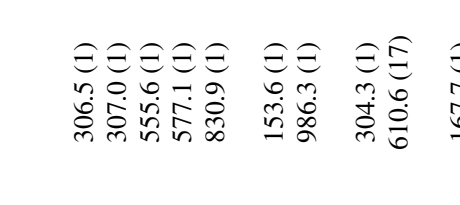 \\
\hline & 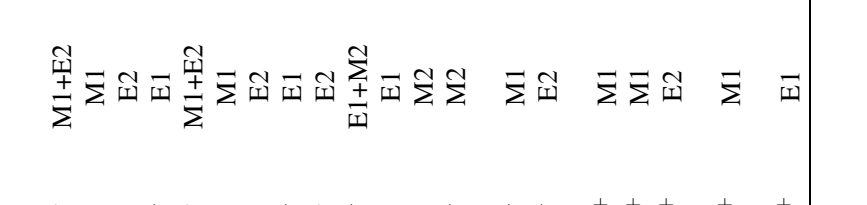 & 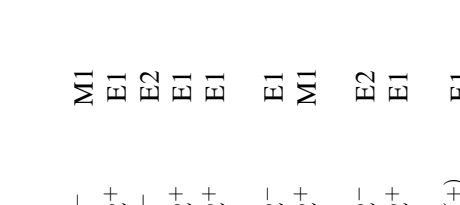 \\
\hline & 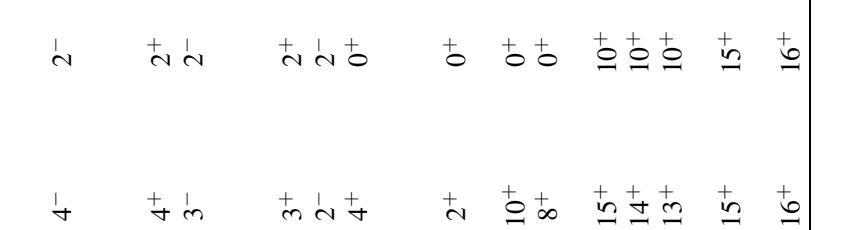 & 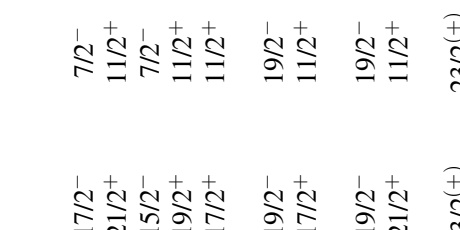 \\
\hline & . $\quad 3=0$ & $\underline{1}$ \\
\hline & 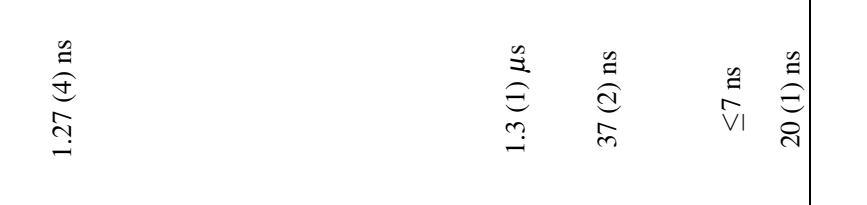 & $\frac{1}{\bar{z}}$ \\
\hline & & \\
\hline
\end{tabular}




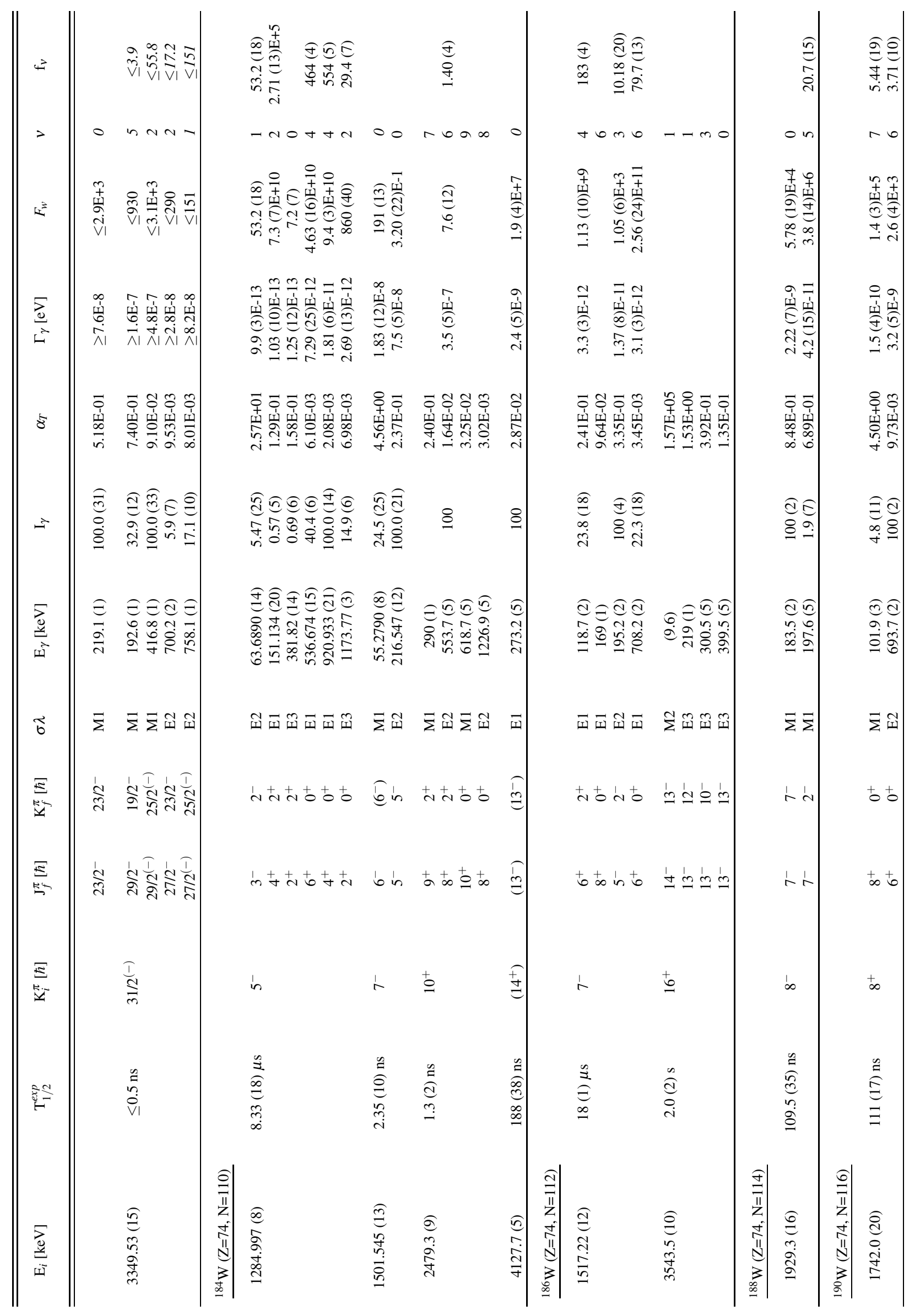




\begin{tabular}{|c|c|c|c|c|c|c|c|c|c|c|c|c|c|c|}
\hline$\rightarrow$ & & & & & 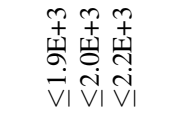 & $\begin{array}{l}\hat{a} \\
\vec{v} 1\end{array}$ & $\begin{array}{l}\stackrel{n}{0} \\
\stackrel{0}{v 1}\end{array}$ & & & & 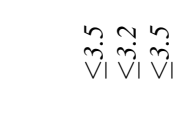 & & & 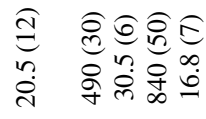 \\
\hline$>$ & 0 & & & & ONNNO & - & $O N$ & & & & n $06 \pm \pm$ & & & $+m+m m$ \\
\hline$x^{3}$ & $\begin{array}{l}n \\
n \\
n\end{array}$ & & & & 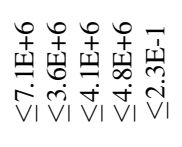 & $\begin{array}{l}\hat{\sigma} \\
\vec{v} 1\end{array}$ & 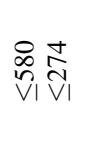 & & & 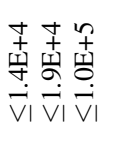 & 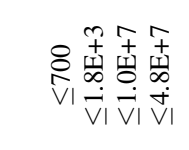 & & & 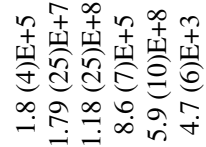 \\
\hline 麄 & 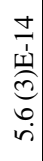 & & & & 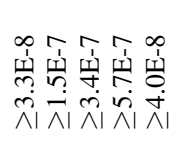 & 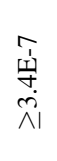 & 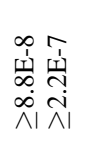 & & & 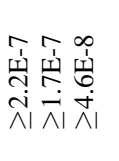 & 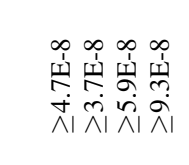 & & & 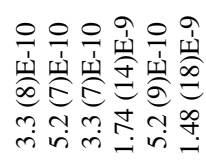 \\
\hline 5 & $\begin{array}{l}\overline{0} \\
+ \\
\oplus \\
0 \\
+ \\
+\end{array}$ & & & & 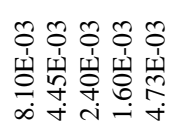 & 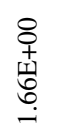 & 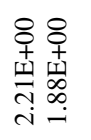 & & & 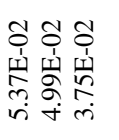 & 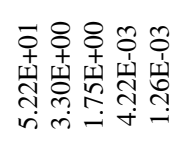 & & & 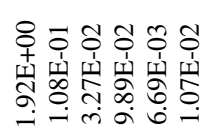 \\
\hline$\Rightarrow$ & $\stackrel{8}{\varnothing}$ & & 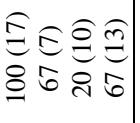 & & 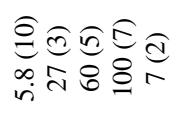 & 8 & $\begin{array}{l}\widehat{0} \hat{0} \\
\dot{\sigma} \\
0\end{array}$ & 8 & & 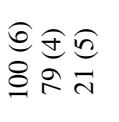 & 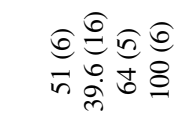 & 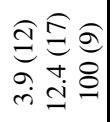 & & 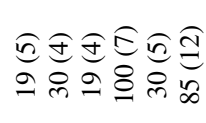 \\
\hline 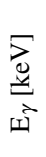 & $\begin{array}{l}\widehat{\infty} \\
0 \\
\dot{a} \\
\vdots\end{array}$ & & 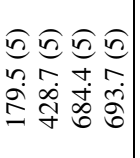 & & 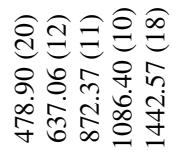 & 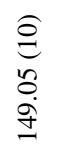 & 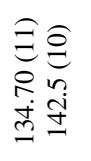 & 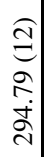 & & 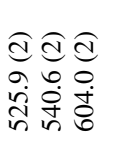 & 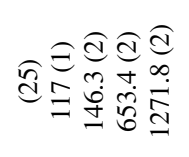 & 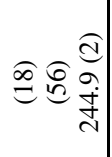 & & 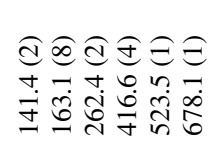 \\
\hline$\widetilde{b}$ & $\stackrel{N}{\Sigma}$ & & & & 피피피포 & $\bar{\Sigma}$ & $\bar{\Sigma} \bar{\Sigma}$ & & & $\bar{\Sigma} \bar{\Sigma} \bar{\Sigma}$ & $\bar{\Sigma} \bar{\Sigma} \bar{\Sigma} \bar{I} \bar{I}$ & & & 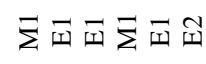 \\
\hline $\begin{array}{l}E \\
E \\
\end{array}$ & $\stackrel{+}{\infty}$ & & 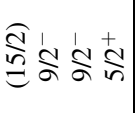 & & 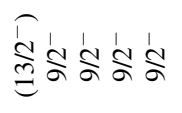 & $\stackrel{+}{\stackrel{N}{I}}$ & $\stackrel{+}{\stackrel{+}{N}} \underset{\mathrm{N}}{\mathbb{S}}$ & నิ & & & 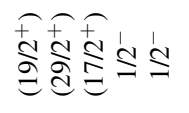 & 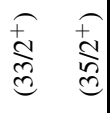 & & ${ }_{\infty}^{+} \sigma_{\infty}^{+} \sigma_{\infty}^{+}$ \\
\hline $\begin{array}{l}E \\
E\end{array}$ & $+\infty$ & & 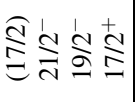 & & 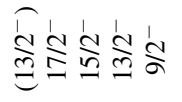 & $\stackrel{+}{\frac{2}{2}}$ & $\stackrel{+}{\stackrel{+}{N}} \stackrel{+}{\stackrel{N}{N}}$ & के & & 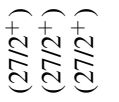 & 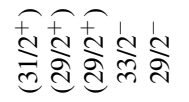 & 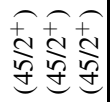 & & 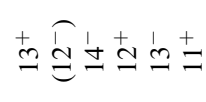 \\
\hline $\begin{array}{l}E \\
\Sigma\end{array}$ & ? & & ลิ & & $\stackrel{+}{\frac{1}{2}}$ & $\stackrel{+}{\stackrel{N}{c}}$ & $\stackrel{+}{\stackrel{\lambda}{N}}$ & $\stackrel{\widehat{S}}{\stackrel{\Xi}{ \pm}}$ & & 先 & 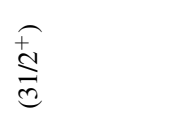 & 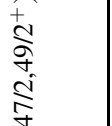 & & $\stackrel{+}{m}$ \\
\hline 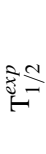 & 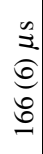 & & 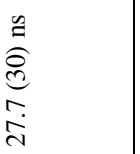 & & 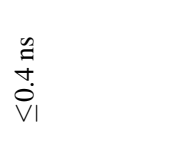 & $\begin{array}{l}n \\
\stackrel{n}{n} \\
\tilde{\delta} \\
v \mid\end{array}$ & $\begin{array}{l}n \\
\vdots \\
n \\
\tilde{V} \\
\dot{v}\end{array}$ & $\stackrel{\varrho}{\Xi}$ & & $\begin{array}{l}\stackrel{\omega}{E} \\
\vec{V} \mid\end{array}$ & $\begin{array}{l}\varrho \\
\vec{V} \mid\end{array}$ & 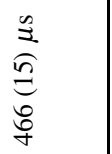 & & 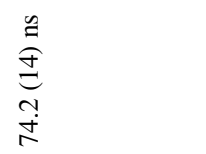 \\
\hline 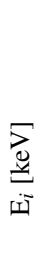 & $\begin{array}{l}\text { త্d } \\
0 \\
\dot{\infty} \\
\infty \\
\infty\end{array}$ & 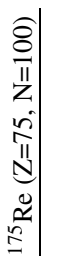 & 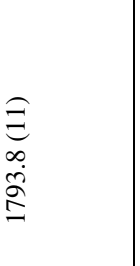 & 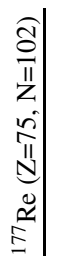 & 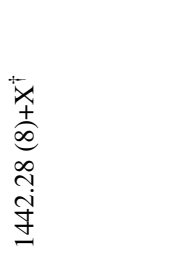 & 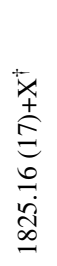 & 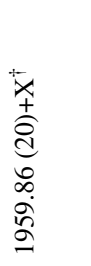 & 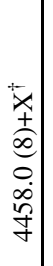 & 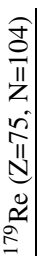 & 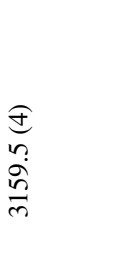 & 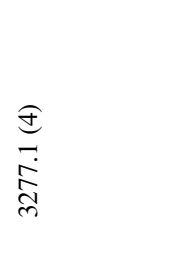 & $\begin{array}{l}0 \\
0 \\
0 \\
\dot{0} \\
\dot{0} \\
i\end{array}$ & 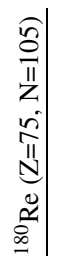 & 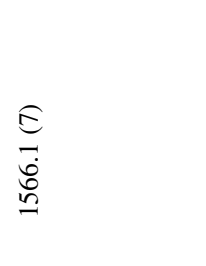 \\
\hline
\end{tabular}




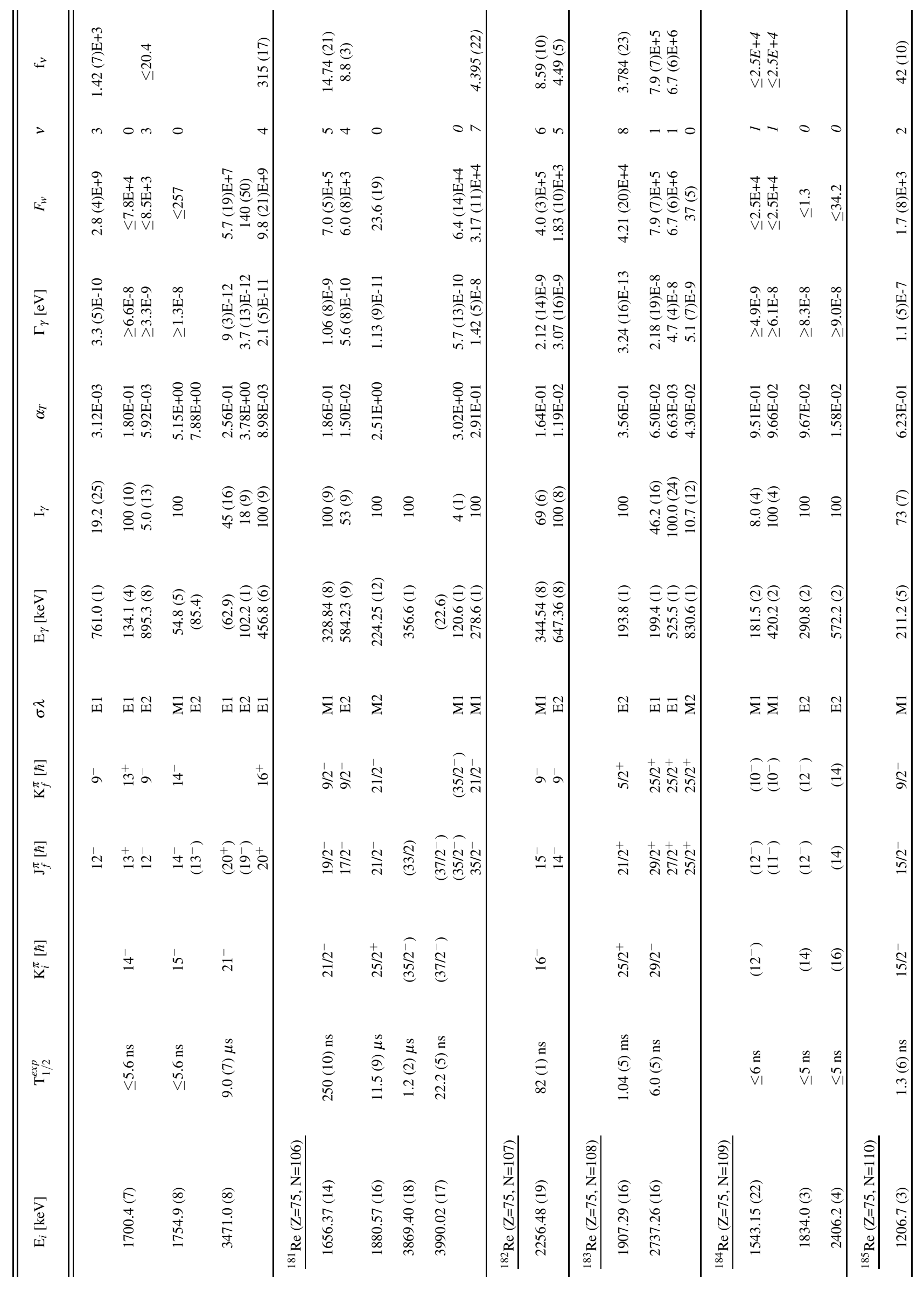




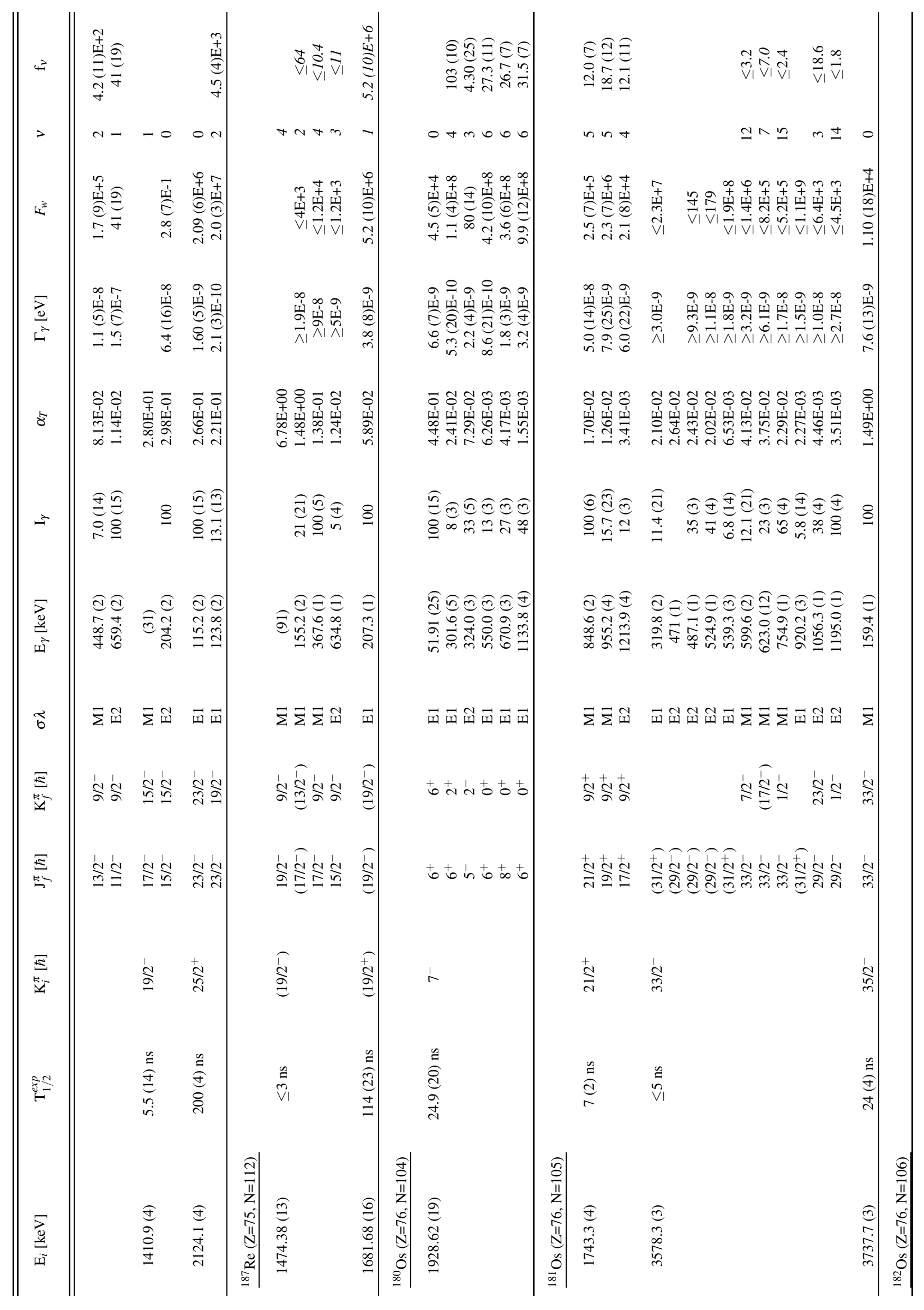




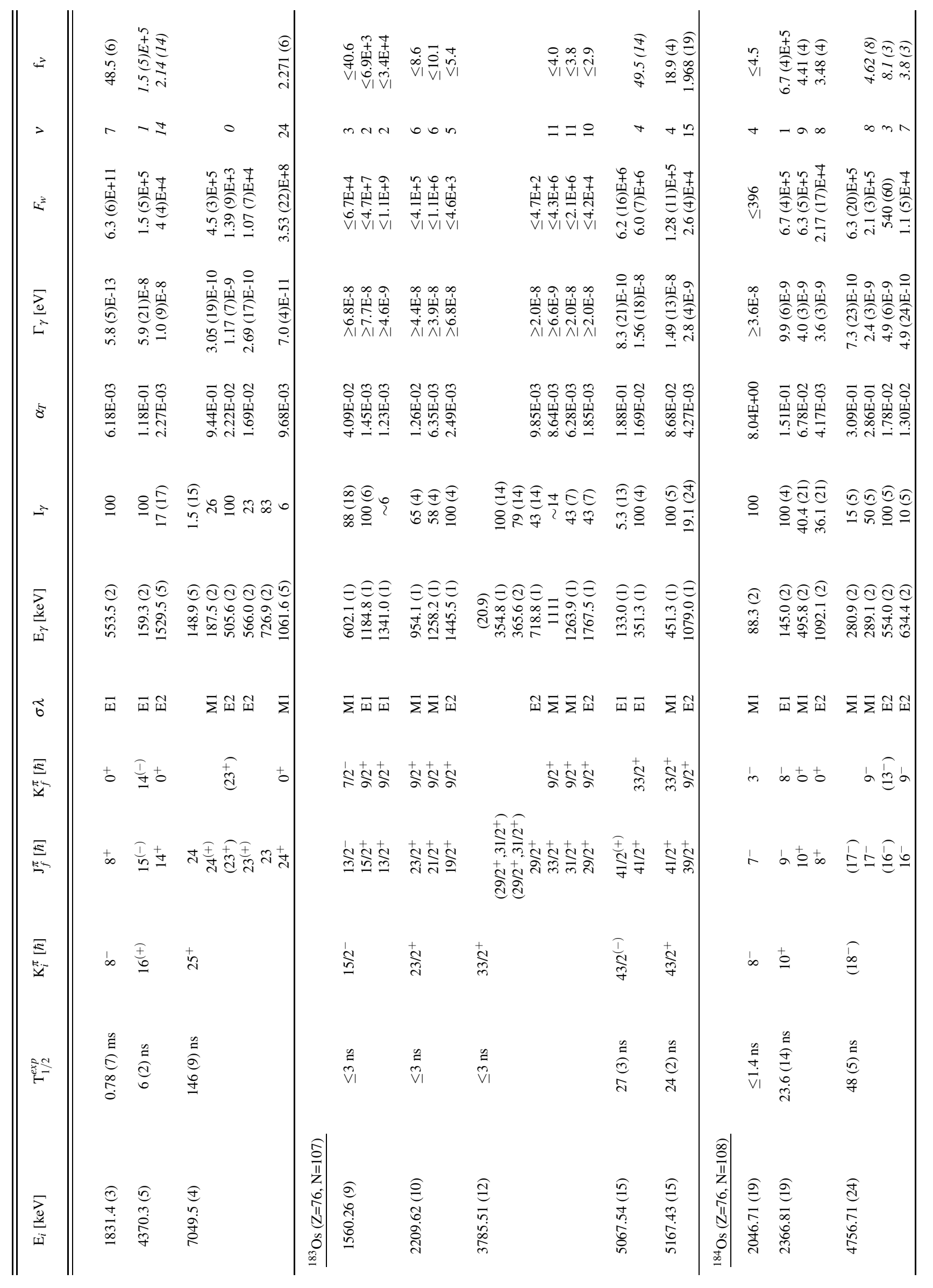




\begin{tabular}{|c|c|c|c|c|c|c|c|c|c|c|c|c|c|c|c|c|}
\hline$\rightarrow$ & $\stackrel{n}{\bar{v} \mid}$ & & & & 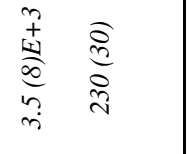 & & 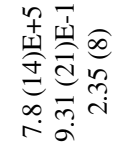 & & & 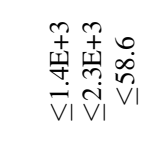 & $\begin{array}{l}\underset{f}{N} \\
\stackrel{n}{m}\end{array}$ & 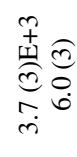 & $\overline{\hat{v}}$ & $\overrightarrow{\hat{D}_{1}} \stackrel{?}{\vec{V}}$ & 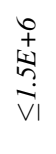 & $\stackrel{0}{\overrightarrow{\mathrm{V}}}$ \\
\hline$>$ & $\infty$ & 0 & 0 & 00 & $\begin{array}{lll}- & \sim & 0\end{array}$ & & $-\infty a$ & & & $O N N T$ & 00 & -4 & $00 \infty$ & -6 & - & $0 \infty$ \\
\hline$x^{3}$ & 字 & 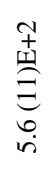 & 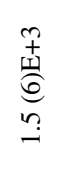 & 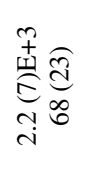 & 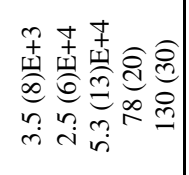 & & 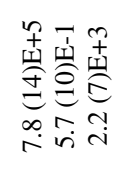 & & & 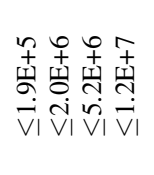 & 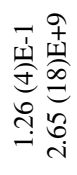 & 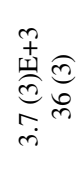 & 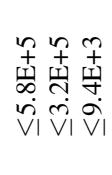 & $\begin{array}{l}\overrightarrow{\dot{\partial}} \stackrel{\sim}{\tilde{\hat{N}}} \\
\vec{v} \mid\end{array}$ & 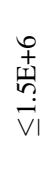 & 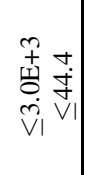 \\
\hline$\frac{5}{\frac{5}{2}}$ & $\begin{array}{l}\text { in } \\
\text { ते } \\
\text { तi }\end{array}$ & 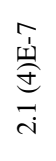 & 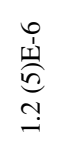 & 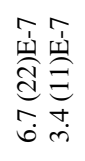 & 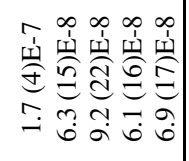 & & 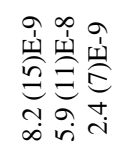 & & & 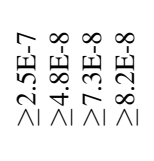 & 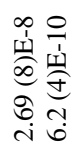 & 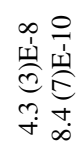 & 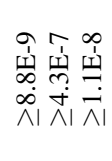 & 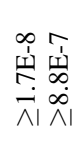 & 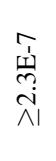 & 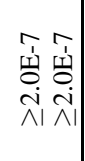 \\
\hline 8 & 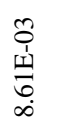 & $\begin{array}{l}8 \\
+ \\
\text { 壱 } \\
\\
-\end{array}$ & 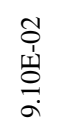 & 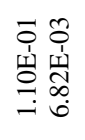 & 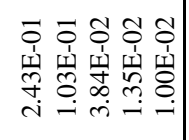 & & 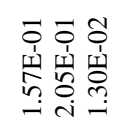 & & & 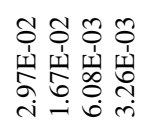 & 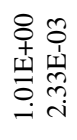 & 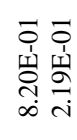 & 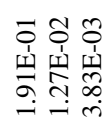 & 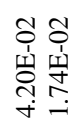 & 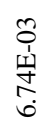 & 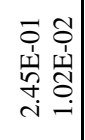 \\
\hline$\Rightarrow$ & 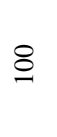 & 8 & 8 & 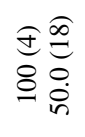 & 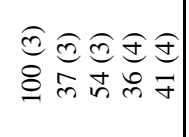 & & 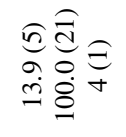 & 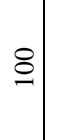 & & 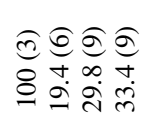 & 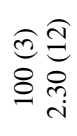 & 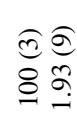 & 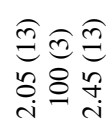 & 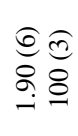 & 8 & 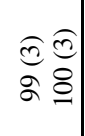 \\
\hline 贫 & 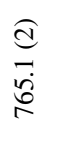 & 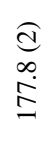 & $\begin{array}{l}\widehat{d} \\
\text { r } \\
\text { d } \\
\text { J }\end{array}$ & 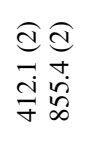 & 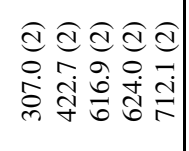 & & 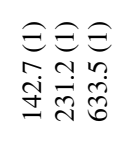 & 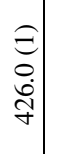 & & 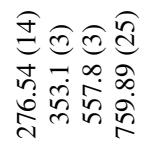 & 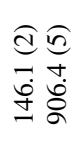 & 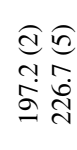 & 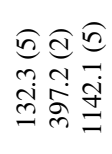 & 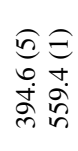 & $\begin{array}{l}\hat{\Xi} \\
\stackrel{.}{n} \\
i\end{array}$ & 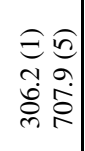 \\
\hline$\widetilde{b}$ & స్త & $\bar{\Sigma}$ & $\bar{\Sigma}$ & $\sum \widetilde{\Sigma}$ & $\bar{\Sigma} \bar{\Sigma} \bar{\Sigma} \widetilde{I}$ & & 피포 포 & & & 포피피 피 & 피피 & $\bar{\Sigma} \widetilde{I}$ & 포피조 & 또포 & $\bar{I}$ & $\bar{\Sigma} \widetilde{x}$ \\
\hline$E$ & to & 总 & $\stackrel{f}{d}$ & $\stackrel{\sqrt{d}}{a} \bar{d}$ & $\begin{array}{ccc}\mp & f & f \\
\stackrel{d}{d} & d & d\end{array}$ & & 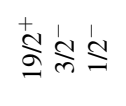 & & & $\stackrel{+}{+} \stackrel{+}{\sim} \stackrel{+}{0}$ & in 0 & in & Һす。 & $\stackrel{+}{\stackrel{+}{=}}=$ & 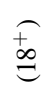 & $\stackrel{\infty}{=} a$ \\
\hline$\sum_{5}^{E}$ & $\stackrel{+}{\infty}$ & $\stackrel{+}{*}$ & $\stackrel{+}{\sim}$ & $\stackrel{+}{*} \stackrel{+}{\sim}$ & 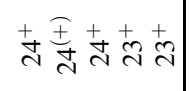 & & 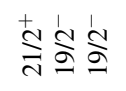 & बิ & & 芓志古古 & in 6 & $\infty$ & ○一大 & 志声 & $\stackrel{+}{=}$ & $\stackrel{\infty}{=}=$ \\
\hline $\begin{array}{l}E \\
E \\
E\end{array}$ & 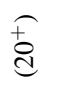 & $\stackrel{\frac{1}{d}}{d}$ & 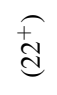 & 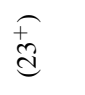 & $\stackrel{\substack{a \\
d}}{a}$ & & 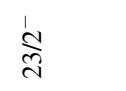 & $\underset{\mathrm{s}}{\stackrel{\mathrm{J}}{\mathrm{J}}}$ & & in & i & a & $\stackrel{\Im}{\varrho}$ & $\stackrel{f}{\stackrel{\infty}{=}}$ & 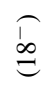 & $\hat{\sigma}$ \\
\hline$\stackrel{5}{5}$ & $\begin{array}{l}\stackrel{n}{E} \\
\stackrel{\vec{v}}{\vec{V}} \mid\end{array}$ & 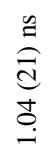 & 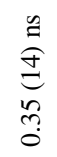 & 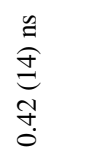 & 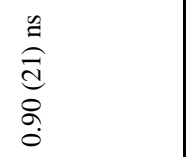 & & $\underset{\substack{a \\
ٍ}}{\stackrel{n}{\sigma}}$ & $\begin{array}{l}0 \\
\stackrel{0}{a} \\
\infty \\
-\infty\end{array}$ & & $\begin{array}{l}\stackrel{\varrho}{\Xi} \\
\vec{V} \mid\end{array}$ & 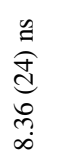 & 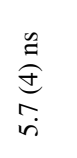 & $\begin{array}{l}\stackrel{a}{\Xi} \\
\vec{V} \mid\end{array}$ & $\begin{array}{l}n \\
\vdots \\
n \\
\tilde{\nabla} \\
\text { | }\end{array}$ & $\begin{array}{l}\mathscr{a} \\
\tilde{V} \\
V_{1}\end{array}$ & $\stackrel{\mathscr{g}}{\vec{\nabla} \mid}$ \\
\hline 离 & 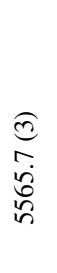 & 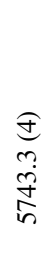 & $\begin{array}{l}\int \\
\vdots \\
0 \\
\infty \\
0\end{array}$ & 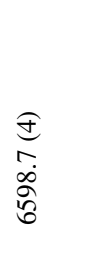 & 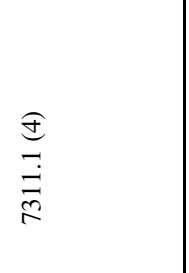 & 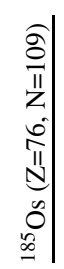 & 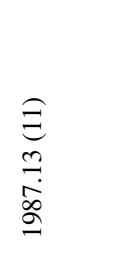 & 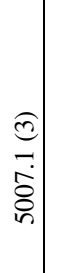 & $\begin{array}{l}0 \\
0 \\
\pi \\
0 \\
0 \\
\pi \\
0 \\
0 \\
0 \\
0\end{array}$ & 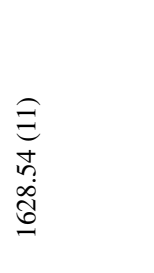 & 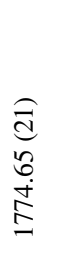 & $\begin{array}{l}\widehat{0} \\
n \\
n \\
\frac{n}{2}\end{array}$ & 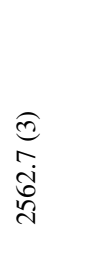 & 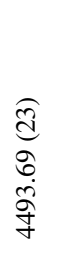 & 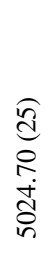 & $\begin{array}{l}\hat{n} \\
\text { òे } \\
\text { हె } \\
\text { nn }\end{array}$ \\
\hline
\end{tabular}




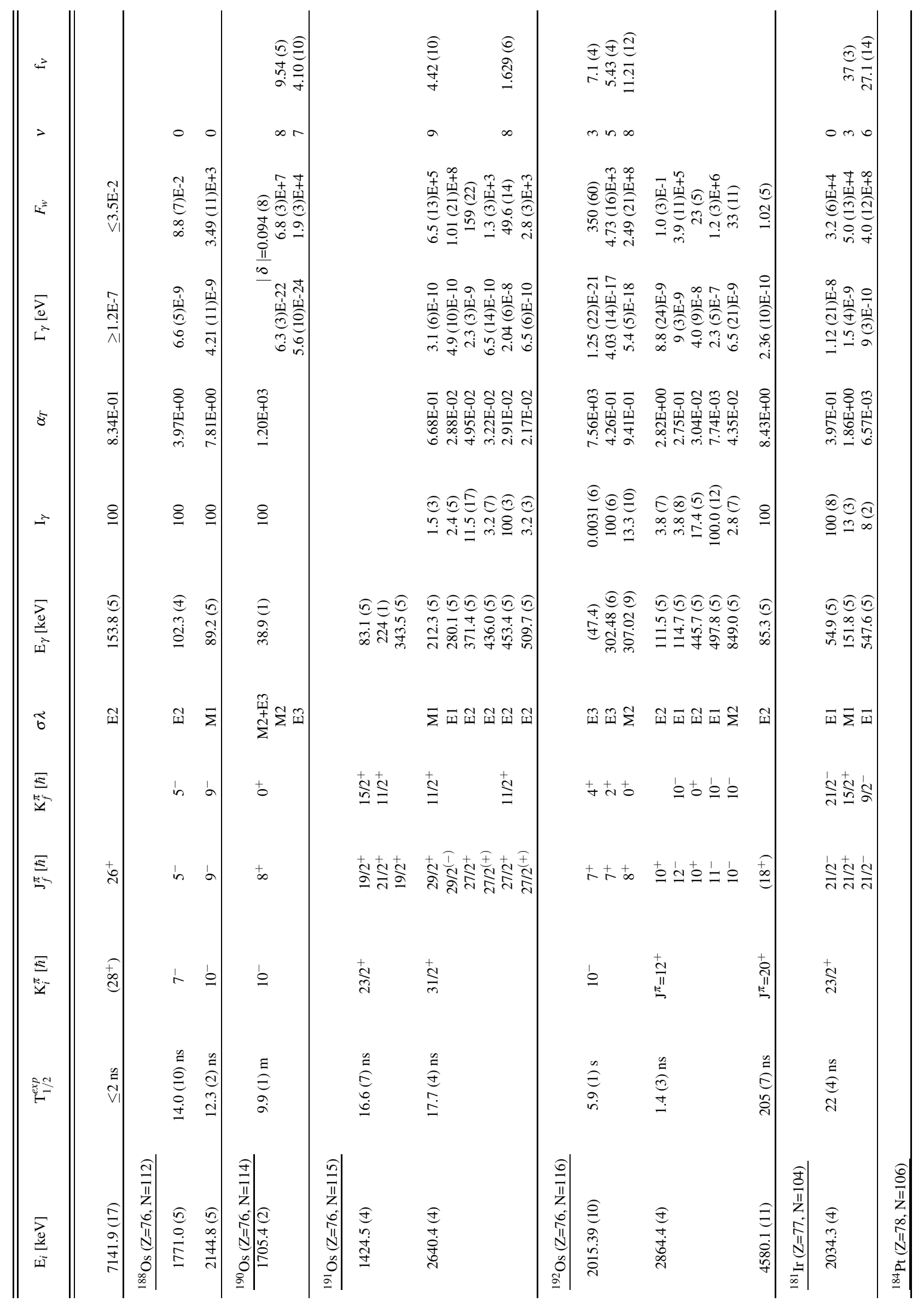




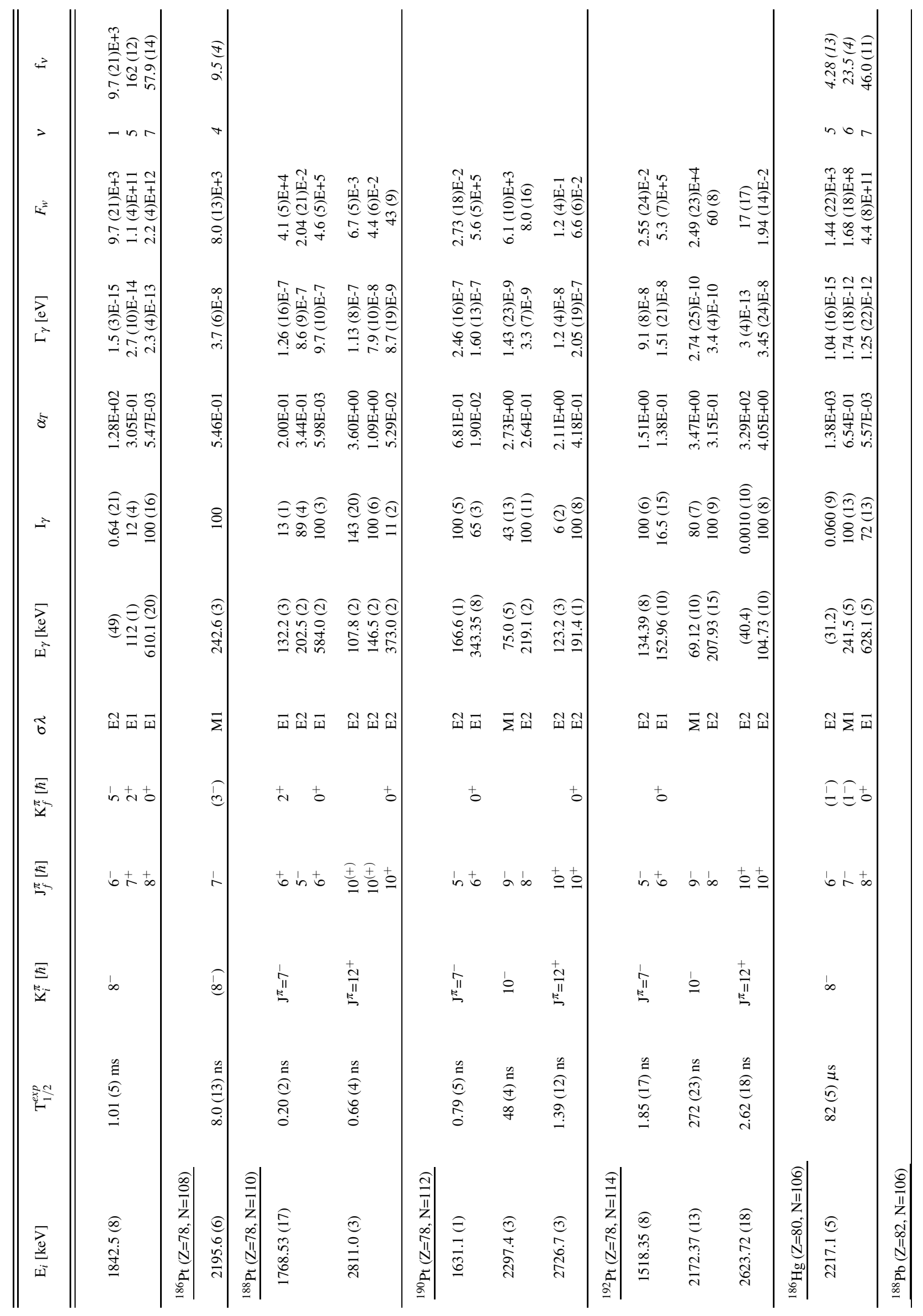




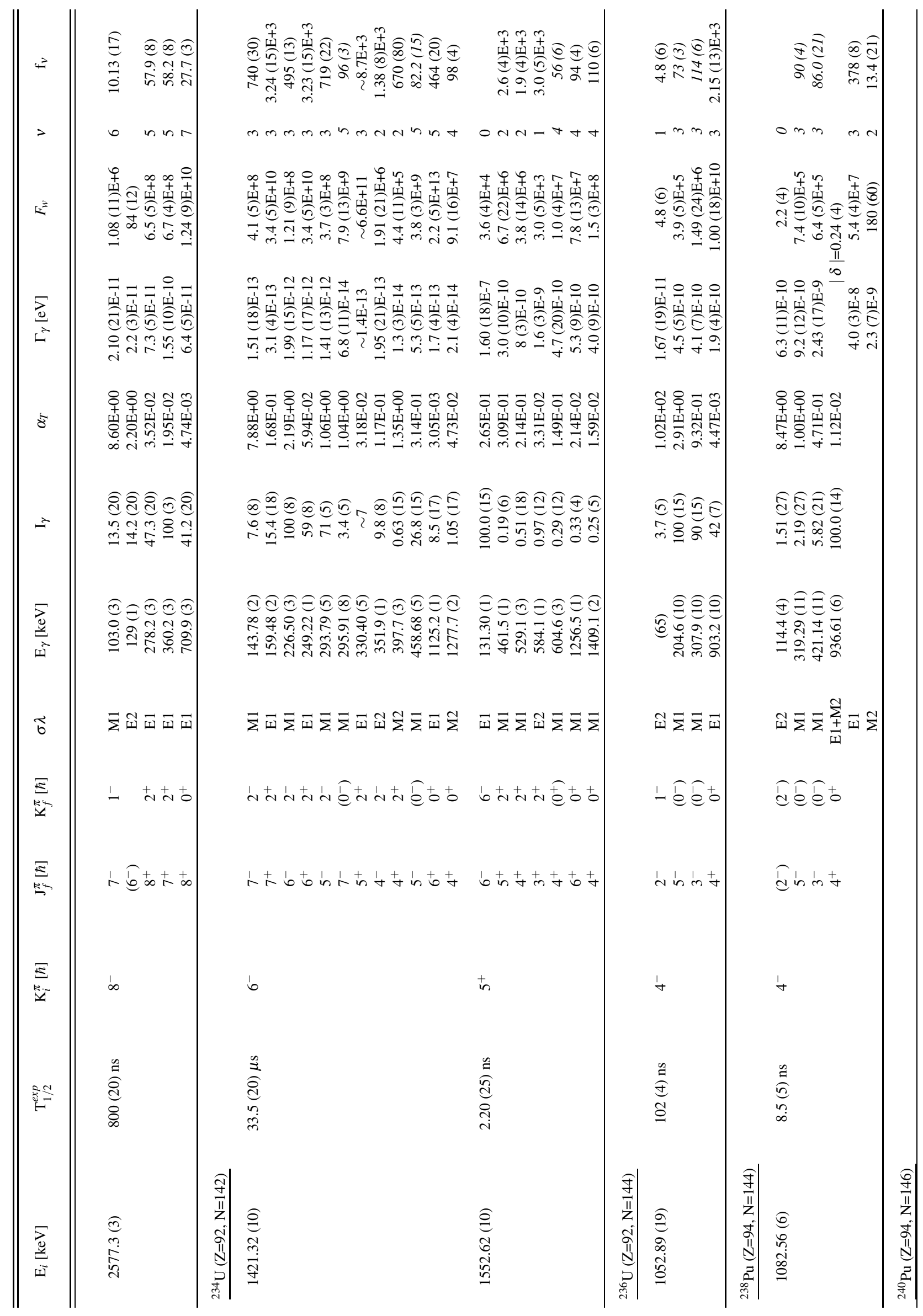




\begin{tabular}{|c|c|c|}
\hline 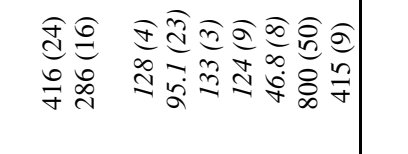 & 玹 & 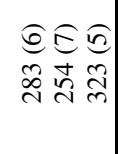 \\
\hline 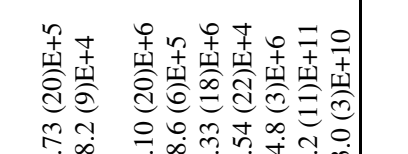 & 譻 & \\
\hline 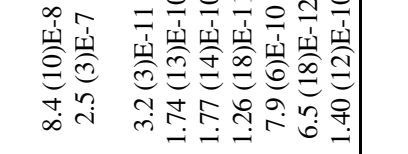 & 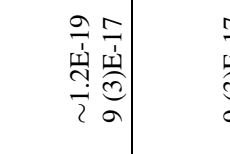 & 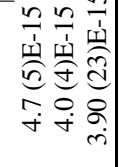 \\
\hline 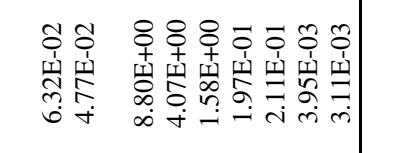 & 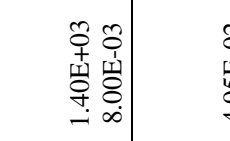 & 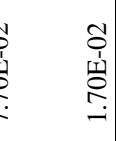 \\
\hline 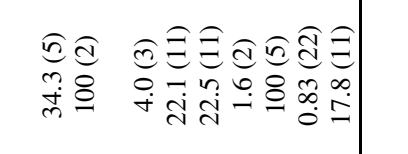 & 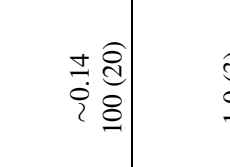 & 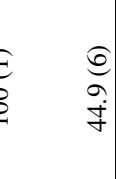 \\
\hline & 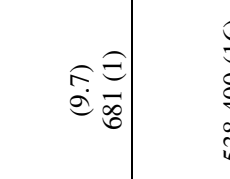 & \\
\hline & $\equiv \bar{\equiv}$ & $\$ 0$ \\
\hline & sot & \\
\hline & 64 & \\
\hline & & \\
\hline 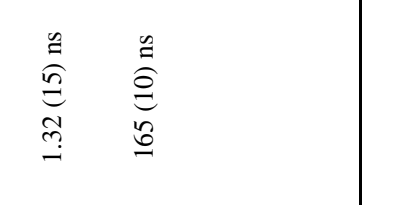 & 蓘 & \\
\hline 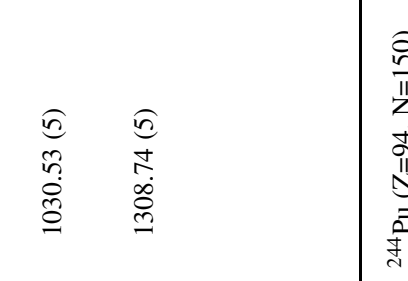 & 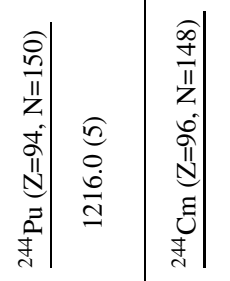 & \\
\hline
\end{tabular}




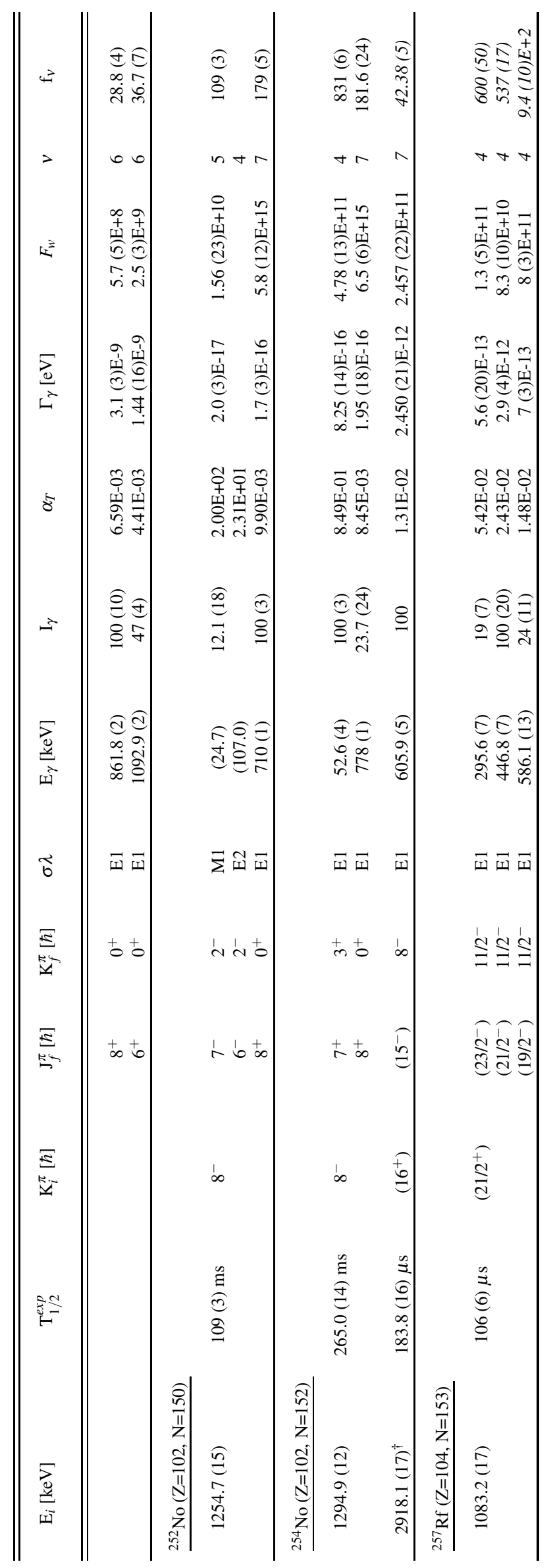


Table 2

$\mathrm{K}$-isomer configurations and bibliography.

\begin{tabular}{ccccc}
\hline \hline $\mathrm{E}_{i}[\mathrm{keV}]$ & $\mathrm{T}_{1 / 2}^{\exp }$ & $\mathrm{K}_{i}^{\pi}[\hbar]$ & Configuration & \\
& & neutrons & protons \\
\hline \hline
\end{tabular}

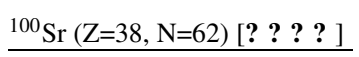

$1618.72(20) \quad 85(7) \mathrm{ns} \quad\left(4^{-}\right)^{*} \quad 3 / 2^{+}[411], 5 / 2^{-}[523]^{\dagger}$

${ }^{99} \mathrm{Y}(\mathrm{Z}=39, \mathrm{~N}=60)[? ? ?$ ? ? ]

$\begin{array}{lrlll}1654.78(23) & \sim 1.4 \mathrm{~ns} & \left(11 / 2^{+}\right)^{*} & 1 / 2^{-}[541], 5 / 2^{-}[532]^{\dagger} & 5 / 2^{+}[422]^{\dagger} \\ 2141.70(22) & 8.6(8) \mu \mathrm{s} & \left(17 / 2^{+}\right) & 3 / 2^{+}[411], 9 / 2^{+}[404]^{\dagger} & 5 / 2^{+}[422]^{\dagger}\end{array}$

${ }^{101} \mathrm{Y}(\mathrm{Z}=39, \mathrm{~N}=62)[? ?]$

$1207.0(16) \quad 0.87(9) \mu \mathrm{s}$

${ }^{108} \mathrm{Zr}(\mathrm{Z}=40, \mathrm{~N}=68)[? ?]$

$2074.5(8) \quad 0.540(30) \mu \mathrm{s} \quad\left(6^{+}\right) \quad 5 / 2^{-}[532], 7 / 2^{-}[523]^{\dagger}$

${ }^{128} \mathrm{Xe}(\mathrm{Z}=54, \mathrm{~N}=74)[? ? ? ?]$

$\begin{array}{rrrr}2228.4(4) & \leq 3.5 \mathrm{~ns} & 5^{-*} & 1 / 2^{+}[400], 9 / 2^{-}[514] \\ 2786.4(4) & 73(3) \mathrm{ns} & 8^{-*} & 7 / 2^{+}[404], 9 / 2^{-}[514]\end{array}$

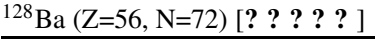

$2395.79(23) \quad 6.1(2) \mathrm{ns} \quad 7^{-*} \quad 7 / 2^{+}[404], 7 / 2^{-}[523]$

${ }^{129} \mathrm{Ba}(\mathrm{Z}=56, \mathrm{~N}=73)[? ? ?]$

$2462.6(2) \quad 47(2) \mathrm{ns} \quad 23 / 2^{+*} \quad 7 / 2^{+}[404], 7 / 2^{-}[523], 9 / 2^{-}[514]$

${ }^{130} \mathrm{Ba}(\mathrm{Z}=56, \mathrm{~N}=74)[?$ ? ? ? ? ? ]

$2475.12(18) \quad 9.4(4) \mathrm{ms} \quad 8^{-} \quad 7 / 2^{+}[404], 9 / 2^{-}[514]$

${ }^{131} \mathrm{La}(\mathrm{Z}=57, \mathrm{~N}=74)[? ?]$
$2121.8(3)$
38 (2) ns
$21 / 2^{-*} \quad 7 / 2^{+}[404], 9 / 2^{-}[514]$
$5 / 2^{+}[413]$

${ }^{130} \mathrm{Ce}(\mathrm{Z}=58, \mathrm{~N}=72)[? ? ?]$

$2453.6(3) \quad 101(9) \mathrm{ns} \quad 7^{-*} \quad 7 / 2^{+}[404], 7 / 2^{-}[523]$

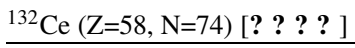

$2341.15(21) \quad 9.4(3) \mathrm{ms} \quad 8^{-*} \quad 7 / 2^{+}[404], 9 / 2^{-}[514]$

${ }^{134} \mathrm{Nd}(\mathrm{Z}=60, \mathrm{~N}=74)[? ? ? ?$ ? $]$

$2293.0(4) \quad 410(30) \mu \mathrm{s} \quad 8^{-*} \quad 7 / 2^{+}[404], 9 / 2^{-}[514]$

${ }^{152} \mathrm{Nd}(\mathrm{Z}=60, \mathrm{~N}=92)[? ? ?]$ 


\begin{tabular}{|c|c|c|c|}
\hline $\mathrm{E}_{i}[\mathrm{keV}]$ & $\mathrm{T}_{1 / 2}^{\exp }$ & $\mathrm{K}_{i}^{\pi}[\hbar]$ & Configuration \\
\hline & & neutrons & protons \\
\hline
\end{tabular}

${ }^{154} \mathrm{Nd}(\mathrm{Z}=60, \mathrm{~N}=94)[? ? ?]$

$1297.90(24) \quad 3.2(3) \mu \mathrm{s} \quad\left(4^{-}\right)^{*} \quad 3 / 2^{-}[521], 5 / 2^{+}[642]^{\dagger}$

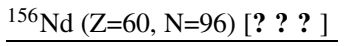

$1431.3(5) \quad 0.37(15) \mu \mathrm{s} \quad\left(5^{-}\right)^{*} \quad 5 / 2^{-}[523], 5 / 2^{+}[642]^{\dagger}$

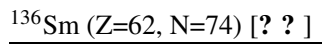

$2264.5(5) \quad 15(1) \mu \mathrm{s} \quad 8^{-*} \quad 7 / 2^{+}[404], 9 / 2^{-}[514]$

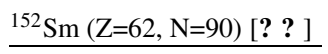

$2120.97(8) \quad 2.4(2) \mathrm{ns} \quad 7^{-*} \quad 3 / 2^{+}[651], 11 / 2^{-}[505]$

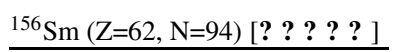

$\begin{array}{llcc}1397.55(9) & 185(7) \mathrm{ns} & 5^{-*} & 5 / 2^{-}[523], 5 / 2^{+}[642] \\ 1515.04(9) & 4.5(2) \mathrm{ns} & 5^{-} & \end{array}$

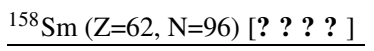

$1279.8(8) \quad 83(12) \mathrm{ns} \quad\left(5^{-}\right)^{*} \quad 5 / 2^{-}[523], 5 / 2^{+}[642]^{\dagger}$

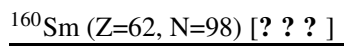

$1361.3(5) \quad 120(46) \mathrm{ns} \quad\left(5^{-}\right) \quad 5 / 2^{-}[523], 5 / 2^{+}[642]^{\dagger}$

${ }^{153} \mathrm{Eu}(\mathrm{Z}=63, \mathrm{~N}=90)[? ?]$

$1771.5(6) \quad 475(10) \mathrm{ns}$
$19 / 2^{-*} \quad 3 / 2^{+}[651], 11 / 2^{-}[505]$
$5 / 2^{+}[413]$
OR
$3 / 2^{+}[402], 11 / 2^{-}[505]$
$5 / 2^{+}[413]$

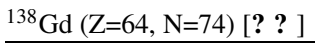

$2232.7(11) \quad 6(1) \mu \mathrm{s} \quad 8^{-*} \quad 7 / 2^{+}[404], 9 / 2^{-}[514]$

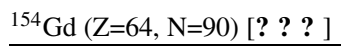

$2137.51(6) \quad 68(4)$
$7^{-*} \quad 3 / 2^{+}[651], 11 / 2^{-}[505]$
OR
$3 / 2^{+}[402], 11 / 2^{-}[505]$

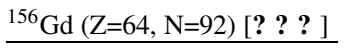

$2137.86(5) \quad 1.3(1) \mu \mathrm{s}$
$7^{-} \quad 3 / 2^{+}[651], 11 / 2^{-}[505]$
OR

$3 / 2^{+}[402], 11 / 2^{-}[505]$

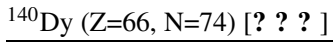

$2166.1(5) \quad 7.0(5) \mu \mathrm{s} \quad 8^{-} \quad 7 / 2^{+}[404], 9 / 2^{-}[514]$

${ }^{162} \mathrm{Dy}(\mathrm{Z}=66, \mathrm{~N}=96)$ [? ? ? ? ] 


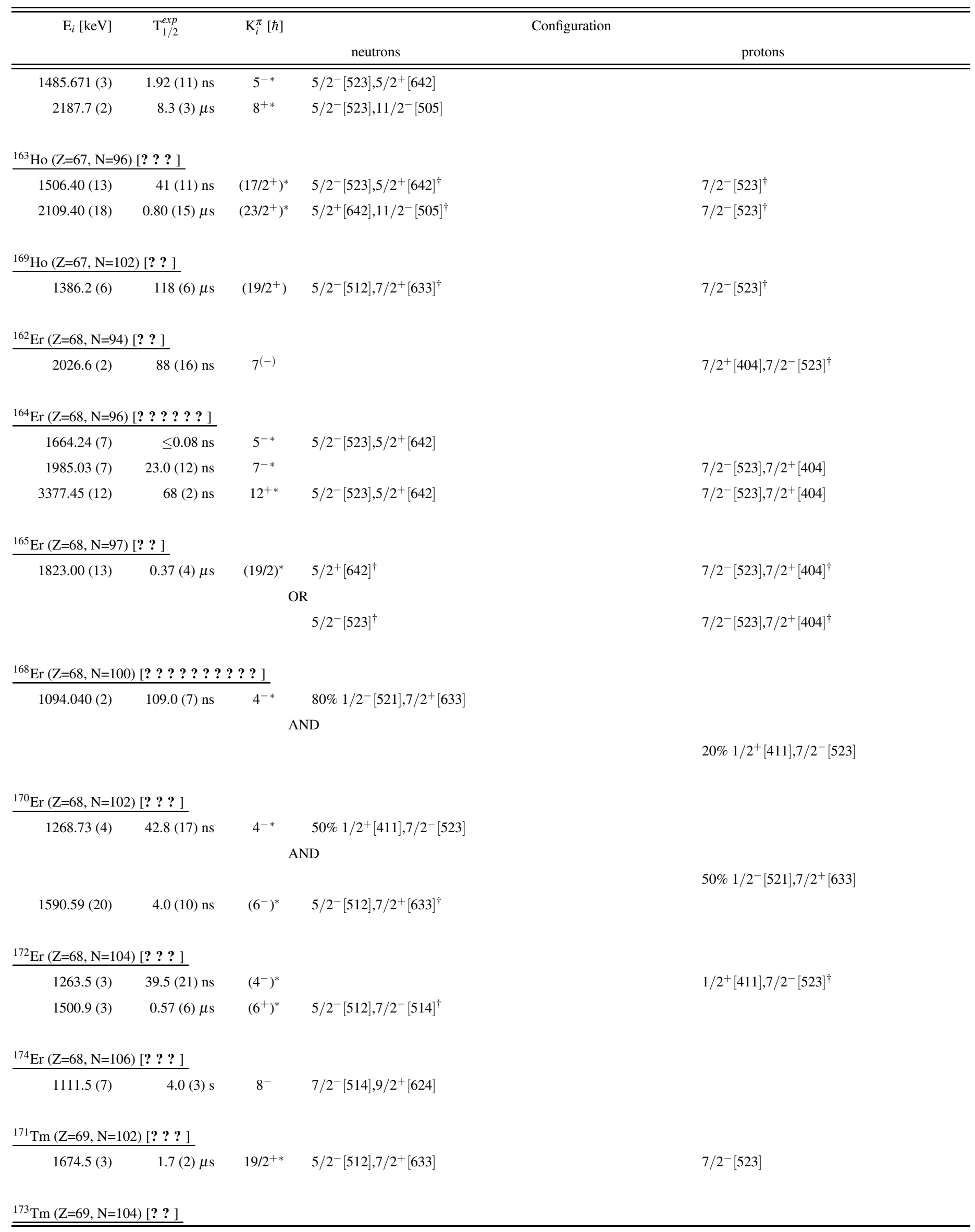




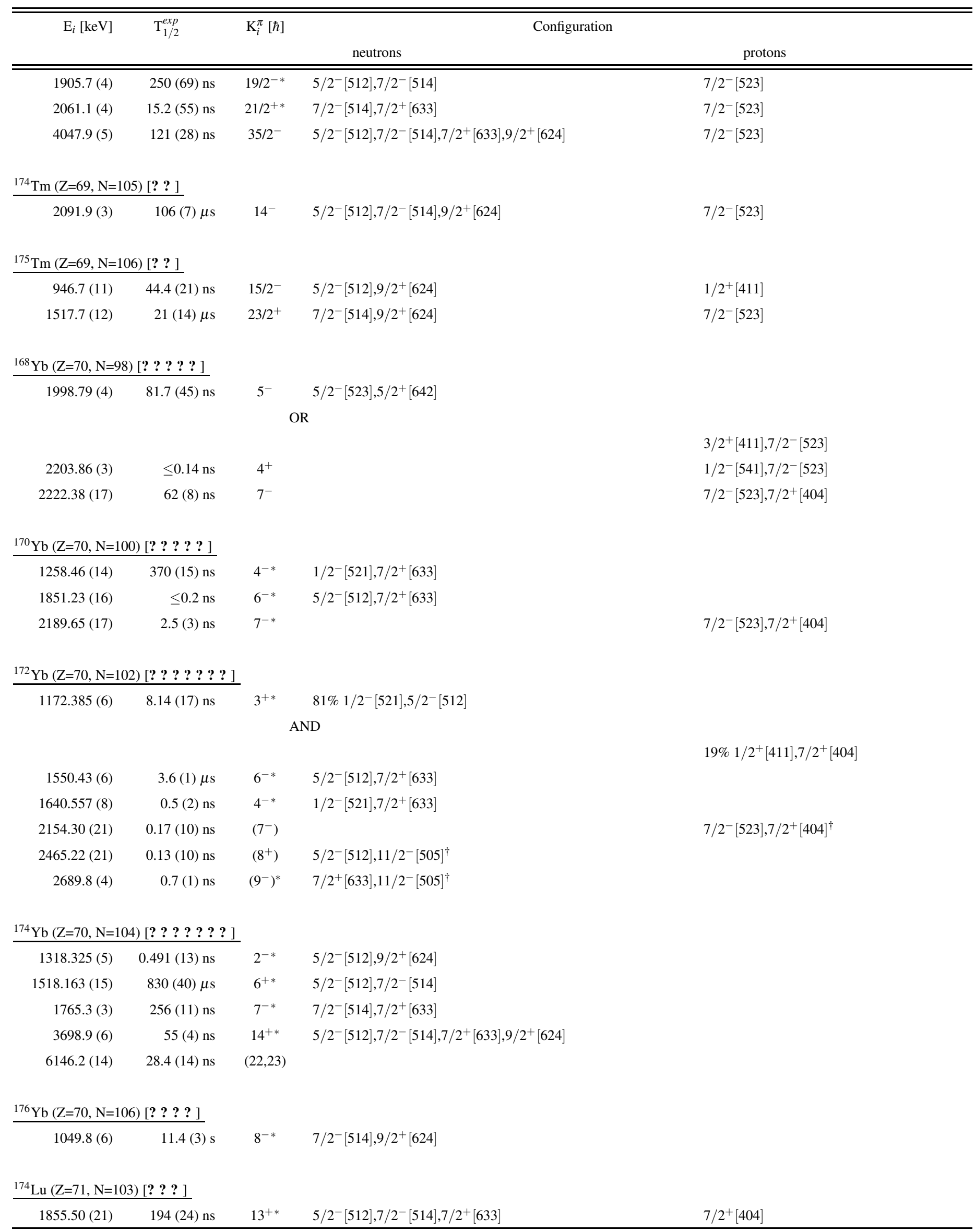




\begin{tabular}{|c|c|c|c|c|}
\hline \multirow[t]{2}{*}{$\mathrm{E}_{i}[\mathrm{keV}]$} & \multirow[t]{2}{*}{$\mathrm{T}_{1 / 2}^{\exp }$} & \multirow[t]{2}{*}{$\mathrm{K}_{i}^{\pi}[\hbar]$} & \multicolumn{2}{|l|}{ Configuration } \\
\hline & & & neutrons & protons \\
\hline $2062.3(6)$ & $38(4) \mathrm{ns}$ & $14^{-*}$ & $5 / 2^{-}[512], 7 / 2^{-}[514], 7 / 2^{+}[633]$ & $9 / 2^{-}[514]$ \\
\hline $2731.9(8)$ & $\leq 2 \mathrm{~ns}$ & $\left(15^{+}\right)^{*}$ & $5 / 2^{-}[512], 7 / 2^{+}[633], 9 / 2^{+}[624]^{\dagger}$ & $9 / 2^{-}[514]^{\dagger}$ \\
\hline $2875.5(9)$ & $\leq 2 \mathrm{~ns}$ & $\left(16^{+}\right)^{*}$ & $7 / 2^{-}[514], 7 / 2^{+}[633], 9 / 2^{+}[624]^{\dagger}$ & $9 / 2^{-}[514]^{\dagger}$ \\
\hline $3740.3(10)$ & $\leq 2 \mathrm{~ns}$ & $\left(19^{+}\right)$ & $1 / 2^{-}[521], 5 / 2^{-}[512], 7 / 2^{-}[514], 7 / 2^{+}[633], 9 / 2^{+}[624]^{\dagger}$ & $9 / 2^{-}[514]^{\dagger}$ \\
\hline $4068.4(12)$ & $97(10) \mathrm{ns}$ & $\left(21^{+}\right)^{*}$ & $5 / 2^{-}[512], 7 / 2^{-}[514], 7 / 2^{+}[633]^{\dagger}$ & $7 / 2^{+}[404], 7 / 2^{-}[523], 9 / 2^{-}[514]^{\dagger}$ \\
\hline $5849.4(14)$ & $242(19) \mathrm{ns}$ & $\left(26^{-}\right)$ & $1 / 2^{-}[521], 5 / 2^{-}[512], 7 / 2^{-}[514], 7 / 2^{+}[633], 9 / 2^{+}[624]^{\dagger}$ & $7 / 2^{+}[404], 7 / 2^{-}[523], 9 / 2^{-}[514]^{\dagger}$ \\
\hline \multicolumn{5}{|c|}{${ }^{175} \mathrm{Lu}(\mathrm{Z}=71, \mathrm{~N}=104)[? ? ? ?]$} \\
\hline $1392.4(4)$ & $957(27) \mu \mathrm{s}$ & $19 / 2^{+}$ & $5 / 2^{-}[512], 7 / 2^{-}[514]$ & $7 / 2^{+}[404]$ \\
\hline \multicolumn{5}{|c|}{${ }^{176} \mathrm{Lu}(\mathrm{Z}=71, \mathrm{~N}=105)[? ?]$} \\
\hline $1514.76(22)$ & $0.31(7) \mu \mathrm{s}$ & $12^{+}$ & $1 / 2^{-}[521], 7 / 2^{-}[514], 9 / 2^{+}[624]$ & $7 / 2^{+}[404]$ \\
\hline $1587.8(6)$ & $40(3) \mu \mathrm{s}$ & $14^{+}$ & $5 / 2^{-}[512], 7 / 2^{-}[514], 9 / 2^{+}[624]$ & $7 / 2^{+}[404]$ \\
\hline \multicolumn{5}{|c|}{${ }^{177} \mathrm{Lu}(\mathrm{Z}=71, \mathrm{~N}=106)[? ? ? ? ?]$} \\
\hline $970.1749(24)$ & $160.44(6) \mathrm{d}$ & $23 / 2^{-*}$ & $7 / 2^{-}[514], 9 / 2^{+}[624]$ & $7 / 2^{+}[404]$ \\
\hline $1324.39(23)$ & $62.4(35) \mathrm{ns}$ & $25 / 2^{+*}$ & $7 / 2^{-}[514], 9 / 2^{+}[624]$ & $9 / 2^{-}[514]$ \\
\hline $2771.6(3)$ & $625(62) \mathrm{ns}$ & $33 / 2^{+*}$ & $7 / 2^{-}[514], 9 / 2^{+}[624]$ & $1 / 2^{+}[411], 7 / 2^{+}[404], 9 / 2^{-}[514]$ \\
\hline $3530.3(4)$ & $6(2) \mu \mathrm{s}$ & $39 / 2^{-}$ & $7 / 2^{-}[514], 9 / 2^{+}[624]$ & $7 / 2^{+}[404], 7 / 2^{-}[523], 9 / 2^{-}[514]$ \\
\hline \multicolumn{5}{|c|}{${ }^{170} \mathrm{Hf}(\mathrm{Z}=72, \mathrm{~N}=98)[? ? ?$ ? ? ] } \\
\hline $1773.36(16)$ & $\leq 5 \mathrm{~ns}$ & $6^{+*}$ & & $5 / 2^{+}[402], 7 / 2^{+}[404]$ \\
\hline $2182.57(21)$ & $23(2) \mathrm{ns}$ & $8^{-*}$ & & $7 / 2^{+}[404], 9 / 2^{-}[514]$ \\
\hline \multicolumn{5}{|c|}{${ }^{171} \mathrm{Hf}(\mathrm{Z}=72, \mathrm{~N}=99)[? ? ? ? ?]$} \\
\hline $1646.34(25)$ & $6.2(14) \mathrm{ns}$ & $19 / 2^{+*}$ & $7 / 2^{+}[633]$ & $5 / 2^{+}[402], 7 / 2^{+}[404]$ \\
\hline $1985.8(4)$ & $18(2) \mathrm{ns}$ & $23 / 2^{-*}$ & $7 / 2^{+}[633]$ & $7 / 2^{+}[404], 9 / 2^{-}[514]$ \\
\hline \multicolumn{5}{|c|}{${ }^{172} \mathrm{Hf}(\mathrm{Z}=72, \mathrm{~N}=100)[? ? ? ? ?$ ? ? ? ? ] } \\
\hline $1418.50(10)$ & $\sim 1 \mathrm{~ns}$ & $4^{-*}$ & $1 / 2^{-}[521], 7 / 2^{+}[633]$ & \\
\hline $1684.69(12)$ & $4.8(4) \mathrm{ns}$ & $6^{+*}$ & & $5 / 2^{+}[402], 7 / 2^{+}[404]$ \\
\hline $1856.71(14)$ & $\leq 16 \mathrm{~ns}$ & $6^{-*}$ & $5 / 2^{-}[512], 7 / 2^{+}[633]$ & \\
\hline $2005.45(14)$ & $163(3) \mathrm{ns}$ & $8^{-*}$ & & $7 / 2^{+}[404], 9 / 2^{-}[514]$ \\
\hline $3319.05(15)$ & $\leq 2 \mathrm{~ns}$ & $12^{+*}$ & $1 / 2^{-}[521], 7 / 2^{+}[633]$ & $7 / 2^{+}[404], 9 / 2^{-}[514]$ \\
\hline $3663.15(20)$ & $\leq 1 \mathrm{~ns}$ & $14^{+*}$ & $5 / 2^{-}[512], 7 / 2^{+}[633]$ & $7 / 2^{+}[404], 9 / 2^{-}[514]$ \\
\hline \multicolumn{5}{|c|}{${ }^{173} \mathrm{Hf}(\mathrm{Z}=72, \mathrm{~N}=101)[? ? ? ?]$} \\
\hline $1700.5(3)$ & $\leq 5 \mathrm{~ns}$ & $19 / 2^{+*}$ & $7 / 2^{+}[633]$ & $5 / 2^{+}[402], 7 / 2^{+}[404]$ \\
\hline $1982.1(5)$ & $19.5(6) \mathrm{ns}$ & $23 / 2^{-*}$ & $7 / 2^{+}[633]$ & $7 / 2^{+}[404], 9 / 2^{-}[514]$ \\
\hline 2814.7 (7) & $\leq 3.5 \mathrm{~ns}$ & $29 / 2^{-*}$ & $1 / 2^{-}[521], 5 / 2^{-}[512], 7 / 2^{+}[633]$ & $7 / 2^{+}[404], 9 / 2^{-}[514]$ \\
\hline \multicolumn{5}{|c|}{${ }^{174} \mathrm{Hf}(\mathrm{Z}=72, \mathrm{~N}=102)[?$} \\
\hline $1549.26(4)$ & $138(4) \mathrm{ns}$ & $6^{+*}$ & & $5 / 2^{+}[402], 7 / 2^{+}[404]$ \\
\hline $1713.49(7)$ & $0.45(10) \mathrm{ns}$ & $6^{-*}$ & $5 / 2^{-}[512], 7 / 2^{+}[633]$ & \\
\hline $1797.59(7)$ & $2.39(4) \mu \mathrm{s}$ & $8^{-*}$ & $7 / 2^{-}[514], 9 / 2^{+}[624]$ & \\
\hline
\end{tabular}




\begin{tabular}{|c|c|c|c|c|}
\hline \multirow[t]{2}{*}{$\mathrm{E}_{i}[\mathrm{keV}]$} & \multirow[t]{2}{*}{$\mathrm{T}_{1 / 2}^{\exp }$} & $\mathrm{K}_{i}^{\pi}[\hbar]$ & \multicolumn{2}{|l|}{ Configuration } \\
\hline & & & neutrons & protons \\
\hline $3312.07(6)$ & $3.7(2) \mu \mathrm{s}$ & $14^{+*}$ & $5 / 2^{-}[512], 7 / 2^{+}[633]$ & $7 / 2^{+}[404], 9 / 2^{-}[514]$ \\
\hline \multicolumn{5}{|c|}{${ }^{175} \mathrm{Hf}(\mathrm{Z}=72, \mathrm{~N}=103)[? ? ? ? ? ? ? ?]$} \\
\hline $1433.54(10)$ & $1.10(8) \mu \mathrm{s}$ & $19 / 2^{+*}$ & $7 / 2^{+}[633]$ & $5 / 2^{+}[402], 7 / 2^{+}[404]$ \\
\hline $1766.45(23)$ & $1.16(11) \mathrm{ns}$ & $23 / 2^{-*}$ & $7 / 2^{+}[633]$ & $7 / 2^{+}[404], 9 / 2^{-}[514]$ \\
\hline $3015.7(4)$ & $1.21(15) \mu \mathrm{s}$ & $35 / 2^{-*}$ & $5 / 2^{-}[512], 7 / 2^{-}[514], 7 / 2^{+}[633]$ & $7 / 2^{+}[404], 9 / 2^{-}[514]$ \\
\hline $3819.6(5)$ & $\leq 7 \mathrm{~ns}$ & $39 / 2^{+*}$ & $7 / 2^{-}[514], 7 / 2^{+}[633], 9 / 2^{+}[624]$ & $7 / 2^{+}[404], 9 / 2^{-}[514]$ \\
\hline $4635.9(6)$ & $1.94(7) \mu \mathrm{s}$ & $45 / 2^{+*}$ & $1 / 2^{-}[521], 5 / 2^{-}[512], 7 / 2^{-}[514], 7 / 2^{+}[633], 9 / 2^{+}[624]$ & $7 / 2^{+}[404], 9 / 2^{-}[514]$ \\
\hline $7455.1(7)$ & $22(2) \mathrm{ns}$ & $57 / 2^{-*}$ & $1 / 2^{-}[521], 5 / 2^{-}[512], 7 / 2^{-}[514], 7 / 2^{+}[633], 9 / 2^{+}[624]$ & $5 / 2^{+}[402], 7 / 2^{+}[404], 7 / 2^{-}[523], 9 / 2^{-}[514]$ \\
\hline $8373.3(8)$ & $\leq 2 \mathrm{~ns}$ & $\left(61 / 2^{+}\right)^{*}$ & $5 / 2^{-}[512], 7 / 2^{-}[514], 5 / 2^{+}[642], 7 / 2^{+}[633], 9 / 2^{+}[624]^{\dagger}$ & $5 / 2^{+}[402], 7 / 2^{+}[404], 7 / 2^{-}[523], 9 / 2^{-}[514]^{\dagger}$ \\
\hline \multicolumn{5}{|c|}{${ }^{176} \mathrm{Hf}(\mathrm{Z}=72, \mathrm{~N}=104)[$ ? ? ? ? ? ? ? ? ? ? ] } \\
\hline \multirow[t]{3}{*}{$1333.16(8)$} & $9.5(2) \mu \mathrm{s}$ & $6^{+*}$ & $61 \% 5 / 2^{-}[512], 7 / 2^{-}[514]$ & \\
\hline & \multicolumn{3}{|r|}{ AND } & \\
\hline & & & & $39 \% 5 / 2^{+}[402], 7 / 2^{+}[404]$ \\
\hline \multirow[t]{3}{*}{$1559.40(9)$} & $9.8(2) \mu \mathrm{s}$ & $8^{-*}$ & $38 \% 7 / 2^{-}[514], 9 / 2^{+}[624]$ & \\
\hline & \multicolumn{3}{|r|}{ AND } & \\
\hline & & & & $62 \% 7 / 2^{+}[404], 9 / 2^{-}[514]$ \\
\hline $2865.8(4)$ & $401(6) \mu \mathrm{s}$ & $14^{-*}$ & $5 / 2^{-}[512], 7 / 2^{-}[514]$ & $7 / 2^{+}[404], 9 / 2^{-}[514]$ \\
\hline $3080.2(6)$ & $0.20(8) \mathrm{ns}$ & $\left(15^{+}\right)^{*}$ & $5 / 2^{-}[512], 9 / 2^{+}[624]^{\dagger}$ & $7 / 2^{+}[404], 9 / 2^{-}[514]^{\dagger}$ \\
\hline $4376.7(9)$ & $34(4) \mathrm{ns}$ & $19^{+}$ & $1 / 2^{-}[521], 5 / 2^{-}[512], 7 / 2^{-}[514], 9 / 2^{+}[624]$ & $7 / 2^{+}[404], 9 / 2^{-}[514]$ \\
\hline $4863.6(9)$ & $43(4) \mu \mathrm{s}$ & $22^{-}$ & $5 / 2^{-}[512], 7 / 2^{-}[514], 7 / 2^{+}[633], 9 / 2^{+}[624]$ & $7 / 2^{+}[404], 9 / 2^{-}[514]$ \\
\hline \multicolumn{5}{|c|}{ 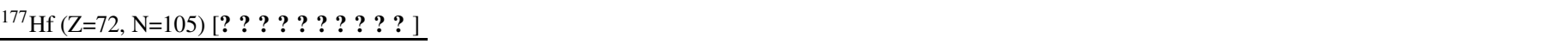 } \\
\hline $1315.4502(8)$ & $1.09(5) \mathrm{s}$ & $23 / 2^{+*}$ & $7 / 2^{-}[514]$ & $7 / 2^{+}[404], 9 / 2^{-}[514]$ \\
\hline $1342.38(20)$ & $55.9(12) \mu \mathrm{s}$ & $\left(19 / 2^{-}\right)$ & $7 / 2^{-}[514]^{\dagger}$ & $5 / 2^{+}[402], 7 / 2^{+}[404]^{\dagger}$ \\
\hline $1713.15(16)$ & $\leq 1 \mathrm{~ns}$ & $25 / 2^{-*}$ & $9 / 2^{+}[624]$ & $7 / 2^{+}[404], 9 / 2^{-}[514]$ \\
\hline $2740.04(15)$ & $51.4(5) \mathrm{m}$ & $37 / 2^{-*}$ & $5 / 2^{-}[512], 7 / 2^{-}[514], 9 / 2^{+}[624]$ & $7 / 2^{+}[404], 9 / 2^{-}[514]$ \\
\hline $3105.72(20)$ & $\leq 1 \mathrm{~ns}$ & $39 / 2^{+*}$ & $7 / 2^{-}[514], 7 / 2^{+}[633], 9 / 2^{+}[624]$ & $7 / 2^{+}[404], 9 / 2^{-}[514]$ \\
\hline \multicolumn{5}{|c|}{${ }^{178} \mathrm{Hf}(\mathrm{Z}=72, \mathrm{~N}=106)[? ? ? ? ? ? ? ? ?$ ? ? ] } \\
\hline \multirow[t]{3}{*}{$1147.398(4)$} & $4.0(2) \mathrm{s}$ & $8^{-*}$ & $64 \% 7 / 2^{-}[514], 9 / 2^{+}[624]$ & \\
\hline & \multicolumn{3}{|c|}{ AND } & \\
\hline & & & & $36 \% 7 / 2^{+}[404], 9 / 2^{-}[514]$ \\
\hline $1553.984(4)$ & $77.5(7) \mathrm{ns}$ & $6^{+*}$ & & $5 / 2^{+}[402], 7 / 2^{+}[404]$ \\
\hline $1636.715(4)$ & $0.4(1) \mathrm{ns}$ & $5^{-*}$ & $1 / 2^{-}[510], 9 / 2^{+}[624]$ & \\
\hline $2446.09(8)$ & $31(1) y$ & $16^{+*}$ & $7 / 2^{-}[514], 9 / 2^{+}[624]$ & $7 / 2^{+}[404], 9 / 2^{-}[514]$ \\
\hline $2572.4(3)$ & $68(2) \mu \mathrm{s}$ & $14^{-*}$ & $7 / 2^{-}[514], 9 / 2^{+}[624]$ & $5 / 2^{+}[402], 7 / 2^{+}[404]$ \\
\hline \multicolumn{5}{|c|}{${ }^{179} \mathrm{Hf}(\mathrm{Z}=72, \mathrm{~N}=107)[?$ ? ? ? ] } \\
\hline $1105.74(16)$ & $25.05(25) \mathrm{d}$ & $25 / 2^{-*}$ & $9 / 2^{+}[624]$ & $7 / 2^{+}[404], 9 / 2^{-}[514]$ \\
\hline $1309.8(6)$ & $3(1) \mathrm{ns}$ & $17 / 2^{+*}$ & $1 / 2^{-}[521], 7 / 2^{-}[514], 9 / 2^{+}[624]$ & \\
\hline $1404.5(11)$ & $4(1) \mathrm{ns}$ & $23 / 2^{+*}$ & $7 / 2^{-}[514]$ & $7 / 2^{+}[404], 9 / 2^{-}[514]$ \\
\hline $1404.5(11)+X^{\dagger}$ & $14(2) \mathrm{ns}$ & $\left(21 / 2^{+}\right)$ & $9 / 2^{+}[624]^{\dagger}$ & $5 / 2^{+}[402], 7 / 2^{+}[404]^{\dagger}$ \\
\hline $2549.6(13)$ & $30(10) \mathrm{ns}$ & $\left(33 / 2^{-}\right)^{*}$ & $1 / 2^{-}[521], 7 / 2^{-}[514], 9 / 2^{+}[624]^{\dagger}$ & $7 / 2^{+}[404], 9 / 2^{-}[514]^{\dagger}$ \\
\hline
\end{tabular}




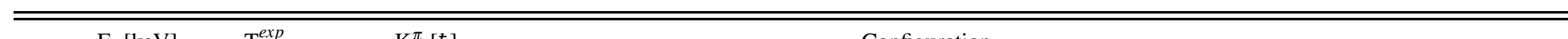

$\begin{array}{llll}\mathrm{E}_{i}[\mathrm{keV}] & \mathrm{T}_{1 / 2}^{\exp } & \mathrm{K}_{i}^{\pi}[\hbar] & \text { Configuration }\end{array}$

neutrons

protons

\begin{tabular}{lllll}
\hline \hline $3439.2(18)$ & $12(6) \mathrm{ns}$ & $\left(39 / 2^{-}\right)$ & $7 / 2^{-}[503], 7 / 2^{-}[514], 9 / 2^{+}[624]^{\dagger}$ & $7 / 2^{+}[404], 9 / 2^{-}[514]^{\dagger}$ \\
$3775.2(21)$ & $15(5) \mu \mathrm{s}$ & $\left(43 / 2^{+}\right)$ & $7 / 2^{-}[514], 9 / 2^{+}[624], 11 / 2^{+}[615]^{\dagger}$ & $7 / 2^{+}[404], 9 / 2^{-}[514]^{\dagger}$
\end{tabular}

${ }^{180} \mathrm{Hf}(\mathrm{Z}=72, \mathrm{~N}=108)[$ ? ? ? ? ? ? ? ? ? ? ? ? ]

$\begin{array}{rrclc}1141.62(6) & 5.47(4) \mathrm{h} & 8^{-*} & & 7 / 2^{+}[404], 9 / 2^{-}[514] \\ 1374.40(5) & 0.545(25) \mu \mathrm{s} & 4^{-*} & 1 / 2^{-}[510], 9 / 2^{+}[624] & 5 / 2^{+}[402], 7 / 2^{+}[404] \\ 1702.7(7) & 2.8(3) \mathrm{ns} & 6^{+*} & & \\ 2426.0(10) & \leq 2 \mathrm{~ns} & 10^{+*} & 9 / 2^{+}[624], 11 / 2^{+}[615] & 7 / 2^{+}[404], 9 / 2^{-}[514] \\ 2486.5(9) & 0.94(11) \mu \mathrm{s} & 12^{+*} & 1 / 2^{-}[510], 9 / 2^{+}[624] & 7 / 2^{+}[404], 9 / 2^{-}[514]^{\dagger} \\ 3597.5(15) & 90(10) \mu \mathrm{s} & \left(18^{-}\right) & 9 / 2^{+}[624], 11 / 2^{+}[615]^{\dagger} & \end{array}$

${ }^{181} \mathrm{Hf}(\mathrm{Z}=72, \mathrm{~N}=109)[? ?]$

$\begin{array}{lrrrr}1040.5(10) & \sim 100 \mu \mathrm{s} & \left(17 / 2^{+}\right)^{*} & 1 / 2^{-}[510]^{\dagger} & 7 / 2^{+}[404], 9 / 2^{-}[514]^{\dagger} \\ 1738.9(11) & 1.5(5) \mathrm{ms} & \left(25 / 2^{-}\right) & 9 / 2^{+}[624]^{\dagger} & 7 / 2^{+}[404], 9 / 2^{-}[514]^{\dagger}\end{array}$

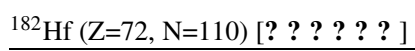

\begin{tabular}{|c|c|c|c|}
\hline $1172.87(18)$ & $61.5(15) \mathrm{m}$ & $8^{-*}$ & $7 / 2^{+}[404], 9 / 2^{-}[514]$ \\
\hline $2571.3(12)$ & $40(10) \mu \mathrm{s}$ & $1 / 2^{-}[510], 11 / 2^{+}[615]^{\dagger}$ & $7 / 2^{+}[404], 9 / 2^{-}[514]^{\dagger}$ \\
\hline
\end{tabular}

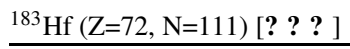

$1464(64) \quad 10(+48-5) \mathrm{s} \quad\left(27 / 2^{-}\right) \quad 11 / 2^{+}[615]^{\dagger} \quad 7 / 2^{+}[404], 9 / 2^{-}[514]^{\dagger}$

${ }^{184} \mathrm{Hf}(\mathrm{Z}=72, \mathrm{~N}=112)[? ? ? ? ?]$

\begin{tabular}{|c|c|c|c|c|}
\hline $1272.2(4)$ & $48(10) \mathrm{s}$ & $8^{-}$ & & $7 / 2^{+}[404], 9 / 2^{-}[514]$ \\
\hline 2477 (10) & $12(+10-4) \mathrm{m}$ & $\left(15^{+}\right)$ & $3 / 2^{-}[512], 11 / 2^{+}[615]^{\dagger}$ & $7 / 2^{+}[404], 9 / 2^{-}[514]^{\dagger}$ \\
\hline
\end{tabular}

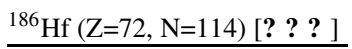

$2968(43) \quad \geq 20 \mathrm{~s} \quad\left(17^{+}\right) \quad 7 / 2^{-}[503], 11 / 2^{+}[615]^{\dagger} \quad 7 / 2^{+}[404], 9 / 2^{-}[514]^{\dagger}$

${ }^{173} \mathrm{Ta}(\mathrm{Z}=73, \mathrm{~N}=100)[? ? ? ?]$

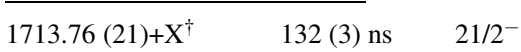

$5 / 2^{+}[402], 7 / 2^{+}[404], 9 / 2^{-}[514]$

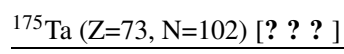

$1279.5(3) \leq 1 \mathrm{~ns}$

$1552.00(19) \quad 5.1(6) \mathrm{ns} \quad 17 / 2^{+*}$

$1566.2(3) \quad 1.95(15) \mu \mathrm{s} \quad 21 / 2^{-*}$

$1729.6(4) \quad 0.9(3) \mathrm{ns} \quad 21 / 2^{+*} \quad 5 / 2^{-}[512], 7 / 2^{+}[633]$

$3526.3(5) \leq 0.5 \mathrm{~ns} \quad\left(33 / 2^{-}\right) \quad 5 / 2^{-}[512], 7 / 2^{-}[514]^{\dagger}$

$3762.0(5) \quad \leq 2 \mathrm{~ns} \quad(35 / 2,37 / 2)^{*}$

$1 / 2^{-}[541], 7 / 2^{+}[404], 9 / 2^{-}[514]$

$5 / 2^{+}[402], 7 / 2^{+}[404], 9 / 2^{-}[514]$

$9 / 2^{-}[514]$

$5 / 2^{+}[402], 7 / 2^{+}[404], 9 / 2^{-}[514]^{\dagger}$

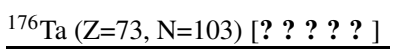

$\begin{array}{rrrll}1370.4(4) & 3.8(4) \mu \mathrm{s} & 14^{-*} & 7 / 2^{+}[633] & 5 / 2^{+}[402], 7 / 2^{+}[404], 9 / 2^{-}[514] \\ 1381.5(6) & 2.0(4) \mathrm{ns} & \left(11^{+}\right)^{*} & 1 / 2^{-}[521], 7 / 2^{-}[514], 7 / 2^{+}[633]^{\dagger} & 7 / 2^{+}[404]^{\dagger} \\ 1431.8(6) & 25(8) \mathrm{ns} & \left(13^{+}\right) & 5 / 2^{-}[512]^{\dagger} & 5 / 2^{+}[402], 7 / 2^{+}[404], 9 / 2^{-}[514]^{\dagger} \\ 1665.7(8) & \leq 1 \mathrm{~ns} & \left(14^{-}\right)^{*} & 5 / 2^{-}[512], 7 / 2^{-}[514], 7 / 2^{+}[633]^{\dagger} & 9 / 2^{-}[514]^{\dagger}\end{array}$




\begin{tabular}{|c|c|c|c|c|}
\hline \multirow[t]{2}{*}{$\mathrm{E}_{i}[\mathrm{keV}]$} & \multirow[t]{2}{*}{$\mathrm{T}_{1 / 2}^{\exp }$} & $\mathrm{K}_{i}^{\pi}[\hbar]$ & \multicolumn{2}{|l|}{ Configuration } \\
\hline & & & neutrons & protons \\
\hline $2770.4(7)$ & $0.97(7) \mathrm{ms}$ & $20^{-*}$ & $5 / 2^{-}[512], 7 / 2^{-}[514], 7 / 2^{+}[633]$ & $5 / 2^{+}[402], 7 / 2^{+}[404], 9 / 2^{-}[514]$ \\
\hline $4422.5(10)$ & $\leq 0.5 \mathrm{~ns}$ & (24) & & \\
\hline $5293.5(10)$ & $\leq 1 \mathrm{~ns}$ & $\left(27^{-}\right)^{*}$ & $5 / 2^{-}[512], 5 / 2^{+}[642], 7 / 2^{-}[514], 7 / 2^{+}[633], 9 / 2^{+}[624]^{\dagger}$ & $5 / 2^{+}[402], 7 / 2^{+}[404], 9 / 2^{-}[514]^{\dagger}$ \\
\hline \multicolumn{5}{|c|}{${ }^{177} \mathrm{Ta}(\mathrm{Z}=73, \mathrm{~N}=104)[$ ? ? ? ? ? ? ? ? ] } \\
\hline $1355.01(19)$ & $5.31(25) \mu \mathrm{s}$ & $21 / 2^{-*}$ & & $5 / 2^{+}[402], 7 / 2^{+}[404], 9 / 2^{-}[514]$ \\
\hline $1475.79(21)$ & $\leq 1.4 \mathrm{~ns}$ & $\left(17 / 2^{-}\right)^{*}$ & $1 / 2^{-}[521], 7 / 2^{-}[514]^{\dagger}$ & $9 / 2^{-}[514]^{\dagger}$ \\
\hline $1522.95(15)$ & $5.5(14) \mathrm{ns}$ & $17 / 2^{+*}$ & & $1 / 2^{-}[541], 7 / 2^{+}[404], 9 / 2^{-}[514]$ \\
\hline $1602.71(18)$ & $\leq 1.4 \mathrm{~ns}$ & $19 / 2^{-*}$ & $7 / 2^{-}[514], 7 / 2^{+}[633]$ & $5 / 2^{+}[402]$ \\
\hline $1698.50(22)$ & $\leq 1 \mathrm{~ns}$ & $23 / 2^{+*}$ & $7 / 2^{-}[514], 7 / 2^{+}[633]$ & $9 / 2^{-}[514]$ \\
\hline $2098.3(3)$ & $\leq 2.8 \mathrm{~ns}$ & $25 / 2^{+*}$ & $7 / 2^{-}[514], 9 / 2^{+}[624]$ & $9 / 2^{-}[514]$ \\
\hline $2671.22(23)$ & $\leq 2.8 \mathrm{~ns}$ & $\left(29 / 2^{+}\right)$ & $5 / 2^{-}[512], 7 / 2^{-}[514]^{\dagger}$ & $1 / 2^{-}[541], 7 / 2^{+}[404], 9 / 2^{-}[514]^{\dagger}$ \\
\hline $2826.46(21)$ & $23(4) \mathrm{ns}$ & $31 / 2^{+*}$ & $1 / 2^{-}[521], 5 / 2^{-}[512], 7 / 2^{-}[514], 9 / 2^{+}[624]$ & $9 / 2^{-}[514]$ \\
\hline $2852.7(3)$ & $46(4) \mathrm{ns}$ & $33 / 2^{-*}$ & $5 / 2^{-}[512], 7 / 2^{-}[514]$ & $5 / 2^{+}[402], 7 / 2^{+}[404], 9 / 2^{-}[514]$ \\
\hline $2956.9(4)$ & $1.8(3) \mathrm{ns}$ & $35 / 2^{+*}$ & $7 / 2^{-}[514], 7 / 2^{+}[633]$ & $5 / 2^{+}[402], 7 / 2^{+}[404], 9 / 2^{-}[514]$ \\
\hline $3287.1(4)$ & $\leq 1.4 \mathrm{~ns}$ & $(37 / 2)^{-*}$ & $5 / 2^{-}[512], 7 / 2^{-}[514], 7 / 2^{+}[633], 9 / 2^{+}[624]^{\dagger}$ & $9 / 2^{-}[514]^{\dagger}$ \\
\hline $4329.4(4)$ & $0.69(21) \mathrm{ns}$ & $43 / 2^{+*}$ & $1 / 2^{-}[521], 5 / 2^{-}[512], 7 / 2^{-}[514], 9 / 2^{+}[624]$ & $5 / 2^{+}[402], 7 / 2^{+}[404], 9 / 2^{-}[514]$ \\
\hline $4570.2(5)$ & $\leq 0.7 \mathrm{~ns}$ & $45 / 2^{-*}$ & $1 / 2^{-}[521], 7 / 2^{-}[514], 7 / 2^{+}[633], 9 / 2^{+}[624]$ & $5 / 2^{+}[402], 7 / 2^{+}[404], 9 / 2^{-}[514]$ \\
\hline $4656.3(5)$ & $133(4) \mu \mathrm{s}$ & $49 / 2^{-}$ & $5 / 2^{-}[512], 7 / 2^{-}[514], 7 / 2^{+}[633], 9 / 2^{+}[624]$ & $5 / 2^{+}[402], 7 / 2^{+}[404], 9 / 2^{-}[514]$ \\
\hline \multicolumn{5}{|c|}{${ }^{178} \mathrm{Ta}(\mathrm{Z}=73, \mathrm{~N}=105)[?$ ? ? ? $]$} \\
\hline $1468.4(4)$ & $59(3) \mathrm{ms}$ & $15^{-*}$ & $5 / 2^{-}[512], 7 / 2^{-}[514], 9 / 2^{+}[624]$ & $9 / 2^{-}[514]$ \\
\hline $1552.5(4)$ & $43(8) \mathrm{ns}$ & $14^{+*}$ & $7 / 2^{-}[514]$ & $5 / 2^{+}[402], 7 / 2^{+}[404], 9 / 2^{-}[514]$ \\
\hline $1892.9(4)$ & $\leq 0.5 \mathrm{~ns}$ & $16^{+*}$ & $7 / 2^{-}[514], 7 / 2^{+}[633], 9 / 2^{+}[624]$ & $9 / 2^{-}[514]$ \\
\hline $2902.9(5)$ & $290(12) \mathrm{ms}$ & $21^{-}$ & $5 / 2^{-}[512], 7 / 2^{-}[514], 9 / 2^{+}[624]$ & $5 / 2^{+}[402], 7 / 2^{+}[404], 9 / 2^{-}[514]$ \\
\hline $2957.7(4)$ & $6.6(7) \mathrm{ns}$ & $19^{+*}$ & $5 / 2^{-}[512], 7 / 2^{-}[514], 9 / 2^{+}[624]$ & $1 / 2^{-}[541], 7 / 2^{+}[404], 9 / 2^{-}[514]$ \\
\hline $3135.0(6)$ & $\leq 0.5 \mathrm{~ns}$ & $22^{+*}$ & $7 / 2^{-}[514], 7 / 2^{+}[633], 9 / 2^{+}[624]$ & $5 / 2^{+}[402], 7 / 2^{+}[404], 9 / 2^{-}[514]$ \\
\hline $3402.7(5)$ & $\leq 1 \mathrm{~ns}$ & $\left(20^{-}\right)^{*}$ & $7 / 2^{-}[514], 7 / 2^{+}[633], 9 / 2^{+}[624]^{\dagger}$ & $1 / 2^{-}[541], 7 / 2^{+}[404], 9 / 2^{-}[514]^{\dagger}$ \\
\hline \multicolumn{5}{|c|}{${ }^{179} \mathrm{Ta}(\mathrm{Z}=73, \mathrm{~N}=106)[? ? ? ? ? ?]$} \\
\hline $1252.13(20)$ & $322(16) \mathrm{ns}$ & $21 / 2^{-*}$ & & $5 / 2^{+}[402], 7 / 2^{+}[404], 9 / 2^{-}[514]$ \\
\hline $1316.8(4)$ & $9.0(2) \mathrm{ms}$ & $25 / 2^{+*}$ & $7 / 2^{-}[514], 9 / 2^{+}[624]$ & $9 / 2^{-}[514]$ \\
\hline $1327.4(4)$ & $1.6(4) \mu \mathrm{s}$ & $23 / 2^{-*}$ & $7 / 2^{-}[514], 9 / 2^{+}[624]$ & $7 / 2^{+}[404]$ \\
\hline \multicolumn{5}{|c|}{$\leq 1 \mathrm{~ns}\left(19 / 2^{+}, 21 / 2^{-}\right)^{*}$} \\
\hline $2638.6(5)$ & $54.1(17) \mathrm{ms}$ & $37 / 2^{+*}$ & $7 / 2^{-}[514], 9 / 2^{+}[624]$ & $5 / 2^{+}[402], 7 / 2^{+}[404], 9 / 2^{-}[514]$ \\
\hline $2791.8(5)$ & $17(3) \mathrm{ns}$ & $33 / 2^{-*}$ & $7 / 2^{-}[514], 9 / 2^{+}[624]$ & $1 / 2^{-}[541], 7 / 2^{+}[404], 9 / 2^{-}[514]$ \\
\hline $5389.8(8)$ & $50(5) \mathrm{ns}$ & $\left(49 / 2^{+}\right)^{*}$ & $5 / 2^{-}[512], 7 / 2^{-}[503], 7 / 2^{-}[514], 9 / 2^{+}[624]^{\dagger}$ & $5 / 2^{+}[402], 7 / 2^{+}[404], 9 / 2^{-}[514]^{\dagger}$ \\
\hline \multicolumn{5}{|c|}{${ }^{180} \mathrm{Ta}(\mathrm{Z}=73, \mathrm{~N}=107)[? ? ? ? ? ? ?]$} \\
\hline $1452.66(13)$ & $31.2(14) \mu \mathrm{s}$ & $15^{-*}$ & $9 / 2^{+}[624]$ & $5 / 2^{+}[402], 7 / 2^{+}[404], 9 / 2^{-}[514]$ \\
\hline $1597.53(11)$ & $\leq 2.4 \mathrm{~ns}$ & $\left(13^{-}\right)^{*}$ & $3 / 2^{-}[512], 5 / 2^{-}[512], 9 / 2^{+}[624]^{\dagger}$ & $9 / 2^{-}[514]^{\dagger}$ \\
\hline $1708.21(18)$ & $\leq 1.0 \mathrm{~ns}$ & $11^{(+) *}$ & $1 / 2^{-}[510], 7 / 2^{-}[514], 9 / 2^{+}[624]^{\dagger}$ & $7 / 2^{+}[404]^{\dagger}$ \\
\hline $1941.53(15)$ & $\leq 1.0 \mathrm{~ns}$ & $\left(14^{+}\right)^{*}$ & $7 / 2^{-}[514]^{\dagger}$ & $5 / 2^{+}[402], 7 / 2^{+}[404], 9 / 2^{-}[514]^{\dagger}$ \\
\hline $2589.48(23)$ & $22(2) \mathrm{ns}$ & $18^{(+) *}$ & $7 / 2^{-}[514], 9 / 2^{+}[624], 11 / 2^{+}[615]$ & $9 / 2^{-}[514]$ \\
\hline $2900.94(24)$ & $\leq 2 \mathrm{~ns}$ & $\left(19^{-}\right)^{*}$ & $1 / 2^{-}[510], 7 / 2^{-}[514], 9 / 2^{+}[624]^{\dagger}$ & $5 / 2^{+}[402], 7 / 2^{+}[404], 9 / 2^{-}[514]^{\dagger}$ \\
\hline
\end{tabular}




\begin{tabular}{|c|c|c|c|c|}
\hline \multirow[t]{2}{*}{$\mathrm{E}_{i}[\mathrm{keV}]$} & \multirow[t]{2}{*}{$\mathrm{T}_{1 / 2}^{\exp }$} & \multirow[t]{2}{*}{$\mathrm{K}_{i}^{\pi}[\hbar]$} & \multicolumn{2}{|c|}{ Configuration } \\
\hline & & & neutrons & protons \\
\hline $3680.2(12)$ & $2.0(5) \mu \mathrm{s}$ & $\left(22^{-}\right)$ & $7 / 2^{-}[503], 7 / 2^{-}[514], 9 / 2^{+}[624]^{\dagger}$ & $5 / 2^{+}[402], 7 / 2^{+}[404], 9 / 2^{-}[514]^{\dagger}$ \\
\hline $4172.2(16)$ & $17(5) \mu \mathrm{s}$ & $\left(24^{+}\right)$ & $7 / 2^{-}[514], 9 / 2^{+}[624], 11 / 2^{+}[615]^{\dagger}$ & $5 / 2^{+}[402], 7 / 2^{+}[404], 9 / 2^{-}[514]^{\dagger}$ \\
\hline \multicolumn{5}{|c|}{${ }^{181} \mathrm{Ta}(\mathrm{Z}=73, \mathrm{~N}=108)[$ ? ? ? ? ] } \\
\hline $1403.0(21)$ & $3.3(1) \mathrm{ns}$ & $\left(17 / 2^{-}\right)^{*}$ & $1 / 2^{-}[510], 7 / 2^{-}[514]^{\dagger}$ & $9 / 2^{-}[514]^{\dagger}$ \\
\hline $1403.0(21)+\mathrm{X}^{\dagger}$ & $140(36) \mathrm{ns}$ & $\left(19 / 2^{+}\right)^{*}$ & $1 / 2^{-}[510], 9 / 2^{+}[624]^{\dagger}$ & $9 / 2^{-}[514]^{\dagger}$ \\
\hline $1483.43(21)$ & $25(2) \mu \mathrm{s}$ & $21 / 2^{-*}$ & & $5 / 2^{+}[402], 7 / 2^{+}[404], 9 / 2^{-}[514]$ \\
\hline $2227.9(9)$ & $210(20) \mu \mathrm{s}$ & $29 / 2^{-}$ & $9 / 2^{+}[624], 11 / 2^{+}[615]$ & $9 / 2^{-}[514]$ \\
\hline \multicolumn{5}{|c|}{${ }^{183} \mathrm{Ta}(\mathrm{Z}=73, \mathrm{~N}=110)[? ? ? ?]$} \\
\hline $1333.4(8)$ & $0.9(3) \mu \mathrm{s}$ & $19 / 2^{+*}$ & $1 / 2^{-}[510], 11 / 2^{+}[615]^{\dagger}$ & $9 / 2^{-}[514]^{\dagger}$ \\
\hline \multicolumn{5}{|c|}{${ }^{185} \mathrm{Ta}(\mathrm{Z}=73, \mathrm{~N}=112)[? ? ? ?]$} \\
\hline $1273.4(4)$ & $11.8(14) \mathrm{ms}$ & $21 / 2^{-}$ & $3 / 2^{-}[512], 11 / 2^{+}[615]$ & $7 / 2^{+}[404]$ \\
\hline \multicolumn{5}{|c|}{${ }^{187} \mathrm{Ta}(\mathrm{Z}=73, \mathrm{~N}=114)[?$ ? ? ] } \\
\hline $1793(10)$ & $22(9) \mathrm{s}$ & $\left(27 / 2^{+}\right)$ & $7 / 2^{-}[503], 11 / 2^{+}[615]^{\dagger}$ & $9 / 2^{-}[514]^{\dagger}$ \\
\hline $2933(14)$ & $\geq 5 \mathrm{~m}$ & $\left(41 / 2^{+}\right)$ & $9 / 2^{-}[505], 11 / 2^{+}[615]^{\dagger}$ & $5 / 2^{+}[402], 7 / 2^{+}[402], 9 / 2^{-}[514]^{\dagger}$ \\
\hline \multicolumn{5}{|c|}{${ }^{173} \mathrm{~W}(\mathrm{Z}=74, \mathrm{~N}=99)[?]$} \\
\hline $0+X^{\dagger}$ & $20(5) \mathrm{ns}$ & $19 / 2^{+}$ & $7 / 2^{+}[633]$ & $5 / 2^{+}[402], 7 / 2^{+}[404]$ \\
\hline \multicolumn{5}{|c|}{${ }^{174} \mathrm{~W}(\mathrm{Z}=74, \mathrm{~N}=100)[? ? ?]$} \\
\hline $2267.8(4)$ & $158(3) \mathrm{ns}$ & $8^{-*}$ & & $7 / 2^{+}[404], 9 / 2^{-}[514]$ \\
\hline $3515.6(4)$ & $128(8) \mathrm{ns}$ & $12^{+*}$ & $1 / 2^{-}[521], 7 / 2^{+}[633]$ & $7 / 2^{+}[404], 9 / 2^{-}[514]$ \\
\hline \multicolumn{5}{|c|}{${ }^{176} \mathrm{~W}(\mathrm{Z}=74, \mathrm{~N}=102)[? ? ? ? ?]$} \\
\hline $3747.0(8)$ & $41(1) \mathrm{ns}$ & $14^{+*}$ & $5 / 2^{-}[512], 7 / 2^{+}[633]$ & $7 / 2^{+}[404], 9 / 2^{-}[514]$ \\
\hline \multicolumn{5}{|c|}{${ }^{177} \mathrm{~W}(\mathrm{Z}=74, \mathrm{~N}=103)[? ?]$} \\
\hline $1645.54(15)$ & $\leq 1 \mathrm{~ns}$ & $19 / 2^{+*}$ & $5 / 2^{-}[512], 7 / 2^{-}[514], 7 / 2^{+}[633]$ & \\
\hline $3202.98(16)$ & $\leq 1 \mathrm{~ns}$ & $29 / 2^{+*}$ & $5 / 2^{-}[512], 7 / 2^{-}[514], 7 / 2^{+}[633]$ & $1 / 2^{-}[541], 9 / 2^{-}[514]$ \\
\hline $3431.24(22)$ & $9(2) \mathrm{ns}$ & $31 / 2^{(+) *}$ & $5 / 2^{-}[512], 7 / 2^{-}[514], 7 / 2^{+}[633]^{\dagger}$ & $5 / 2^{+}[402], 7 / 2^{+}[404]^{\dagger}$ \\
\hline \multicolumn{5}{|c|}{${ }^{178} \mathrm{~W}(\mathrm{Z}=74, \mathrm{~N}=104)[?$ ? ? ? ? ? ] } \\
\hline $1664.94(11)$ & $3.0(4) \mathrm{ns}$ & $6^{+*}$ & $5 / 2^{-}[512], 7 / 2^{-}[514]$ & \\
\hline $1738.70(12)$ & $9.6(5) \mathrm{ns}$ & $7^{-*}$ & $7 / 2^{-}[514], 7 / 2^{+}[633]$ & \\
\hline $3053.81(13)$ & $\leq 2 \mathrm{~ns}$ & $11^{-*}$ & $1 / 2^{-}[521], 5 / 2^{-}[512], 7 / 2^{-}[514], 9 / 2^{+}[624]$ & \\
\hline \multirow[t]{2}{*}{$3235.34(12)$} & $\leq 1 \mathrm{~ns}$ & $12^{+*}$ & $1 / 2^{-}[521], 7 / 2^{-}[514], 7 / 2^{+}[633], 9 / 2^{+}[624]$ or & \\
\hline & & & $5 / 2^{-}[512], 7 / 2^{-}[514]$ & $5 / 2^{+}[402], 7 / 2^{+}[404]$ \\
\hline $3525.53(15)$ & $\leq 1 \mathrm{~ns}$ & $13^{-*}$ & $7 / 2^{-}[514], 7 / 2^{+}[633]$ & $5 / 2^{+}[402], 7 / 2^{+}[404]$ \\
\hline $3593.63(18)$ & $3(1) \mathrm{ns}$ & $14^{-*}$ & $5 / 2^{-}[512], 7 / 2^{-}[514]$ & $7 / 2^{+}[404], 9 / 2^{-}[514]$ \\
\hline $3654.93(19)$ & $30(1) \mathrm{ns}$ & $15^{+*}$ & $7 / 2^{-}[514], 7 / 2^{+}[633]$ & $7 / 2^{+}[404], 9 / 2^{-}[514]$ \\
\hline $4879.72(19)$ & $\leq 3 \mathrm{~ns}$ & $18^{-*}$ & $7 / 2^{-}[514], 7 / 2^{+}[633]$ & $1 / 2^{-}[541], 5 / 2^{+}[402], 7 / 2^{+}[404], 9 / 2^{-}[514]$ \\
\hline $5313.7(3)$ & $64(2) \mathrm{ns}$ & $21^{-*}$ & $5 / 2^{-}[512], 7 / 2^{-}[514], 7 / 2^{+}[633], 9 / 2^{+}[624]$ & $5 / 2^{+}[402], 9 / 2^{-}[514]$ \\
\hline
\end{tabular}




\begin{tabular}{|c|c|c|c|c|}
\hline \multirow[t]{2}{*}{$\mathrm{E}_{i}[\mathrm{keV}]$} & \multirow[t]{2}{*}{$\mathrm{T}_{1 / 2}^{\exp }$} & \multirow[t]{2}{*}{$\mathrm{K}_{i}^{\pi}[\hbar]$} & \multicolumn{2}{|l|}{ Configuration } \\
\hline & & & neutrons & protons \\
\hline $6572.7(3)$ & $220(10) \mathrm{ns}$ & $25^{+*}$ & $5 / 2^{-}[512], 7 / 2^{-}[514], 7 / 2^{+}[633], 9 / 2^{+}[624]$ & $1 / 2^{-}[541], 5 / 2^{+}[402], 7 / 2^{+}[404], 9 / 2^{-}[514]$ \\
\hline $8148.4(4)$ & $\leq 5 \mathrm{~ns}$ & $28^{-*}$ & $5 / 2^{-}[512], 7 / 2^{-}[514], 7 / 2^{+}[633], 9 / 2^{+}[624]$ & $1 / 2^{-}[541], 7 / 2^{+}[404], 9 / 2^{-}[514], 11 / 2^{-}[505]$ \\
\hline $8800.3(4)$ & $\leq 1 \mathrm{~ns}$ & $30^{+*}$ & $5 / 2^{-}[512], 7 / 2^{-}[514], 7 / 2^{+}[633], 9 / 2^{+}[624]$ & $5 / 2^{+}[402], 7 / 2^{+}[404], 9 / 2^{-}[514], 11 / 2^{-}[505]$ \\
\hline $8905.6(4)$ & $\leq 1 \mathrm{~ns}$ & $\left(29^{+}\right)^{*}$ & $1 / 2^{-}[521], 5 / 2^{-}[512], 7 / 2^{-}[514], 7 / 2^{-}[503], 7 / 2^{+}[633], 9 / 2^{+}[624]^{\dagger}$ & $1 / 2^{-}[541], 5 / 2^{+}[402], 7 / 2^{+}[404], 9 / 2^{-}[514]^{\dagger}$ \\
\hline $11075.5(5)$ & $\leq 1 \mathrm{~ns}$ & $\left(34^{+}\right)$ & $1 / 2^{-}[521], 5 / 2^{-}[512], 7 / 2^{-}[514], 7 / 2^{-}[503], 7 / 2^{+}[633], 9 / 2^{+}[624]^{\dagger}$ & $5 / 2^{+}[402], 7 / 2^{+}[404], 9 / 2^{-}[514], 11 / 2^{-}[505]^{\dagger}$ \\
\hline \multicolumn{5}{|c|}{${ }^{179} \mathrm{~W}(\mathrm{Z}=74, \mathrm{~N}=105)[? ? ? ? ?]$} \\
\hline $1631.90(8)$ & $390(30) \mathrm{ns}$ & $21 / 2^{+*}$ & $5 / 2^{-}[512], 7 / 2^{-}[514], 9 / 2^{+}[624]$ & \\
\hline $1832.28(14)$ & $\leq 0.5 \mathrm{~ns}$ & $23 / 2^{-*}$ & $7 / 2^{-}[514], 7 / 2^{+}[633], 9 / 2^{+}[624]$ & \\
\hline $2012.10(14)$ & $\leq 1.0 \mathrm{~ns}$ & $(23 / 2)^{+*}$ & $7 / 2^{-}[514]^{\dagger}$ & $7 / 2^{+}[404], 9 / 2^{-}[514]^{\dagger}$ \\
\hline $2088.50(22)$ & $\leq 0.5 \mathrm{~ns}$ & $\left(23 / 2^{-}\right)^{*}$ & $9 / 2^{+}[624]^{\dagger}$ & $5 / 2^{+}[402], 9 / 2^{-}[514]^{\dagger}$ \\
\hline $3224.98(17)$ & $\leq 0.5 \mathrm{~ns}$ & $\left(31 / 2^{+}\right)^{*}$ & $5 / 2^{-}[512], 7 / 2^{-}[514], 9 / 2^{+}[624]^{\dagger}$ & $1 / 2^{-}[541], 9 / 2^{-}[514]^{\dagger}$ \\
\hline $3348.41(14)$ & $750(80) \mathrm{ns}$ & $35 / 2^{-*}$ & $5 / 2^{-}[512], 7 / 2^{-}[514], 9 / 2^{+}[624]$ & $5 / 2^{+}[402], 9 / 2^{-}[514]$ \\
\hline $3582.60(22)$ & $\leq 0.5 \mathrm{~ns}$ & $37 / 2^{+*}$ & $7 / 2^{-}[514], 7 / 2^{+}[633], 9 / 2^{+}[624]$ & $5 / 2^{+}[402], 9 / 2^{-}[514]$ \\
\hline $3596.53(21)$ & $0.7(2) \mathrm{ns}$ & $37 / 2^{-*}$ & $5 / 2^{-}[512], 7 / 2^{-}[514], 9 / 2^{+}[624]$ & $7 / 2^{+}[404], 9 / 2^{-}[514]$ \\
\hline $3778.12(24)$ & $\leq 0.5 \mathrm{~ns}$ & $39 / 2^{+*}$ & $7 / 2^{-}[514], 7 / 2^{+}[633], 9 / 2^{+}[624]$ & $7 / 2^{+}[404], 9 / 2^{-}[514]$ \\
\hline $4609.8(3)$ & $0.7(2) \mathrm{ns}$ & $43 / 2^{+*}$ & $5 / 2^{-}[512], 7 / 2^{-}[514], 9 / 2^{+}[624]$ & $1 / 2^{-}[541], 5 / 2^{+}[402], 7 / 2^{+}[404], 9 / 2^{-}[514]$ \\
\hline \multicolumn{5}{|c|}{${ }^{180} \mathrm{~W}(\mathrm{Z}=74, \mathrm{~N}=106)[$ ? ? ? ? ? ? ? ? ? ?] } \\
\hline $1529.01(3)$ & $5.47(9) \mathrm{ms}$ & $8^{-*}$ & $7 / 2^{-}[514], 9 / 2^{+}[624]$ & \\
\hline $1639.768(20)$ & $19.2(3) \mathrm{ns}$ & $5^{-*}$ & $1 / 2^{-}[521], 9 / 2^{+}[624]$ & \\
\hline $3264.7(3)$ & $2.33(19) \mu \mathrm{s}$ & $14^{-}$ & $7 / 2^{-}[514], 9 / 2^{+}[624]$ & $5 / 2^{+}[402], 7 / 2^{+}[404]$ \\
\hline $3389.7(6)$ & $14.9(6) \mathrm{ns}$ & $15^{+}$ & $7 / 2^{-}[514], 9 / 2^{+}[624]$ & $5 / 2^{+}[402], 9 / 2^{-}[514]$ \\
\hline $3547.7(8)$ & $25.1(5) \mathrm{ns}$ & $16^{+*}$ & $7 / 2^{-}[514], 9 / 2^{+}[624]$ & $7 / 2^{+}[404], 9 / 2^{-}[514]$ \\
\hline $3745.1(8)$ & $\leq 1 \mathrm{~ns}$ & $\left(15^{-}\right)^{*}$ & $7 / 2^{+}[633], 9 / 2^{+}[624]^{\dagger}$ & $5 / 2^{+}[402], 9 / 2^{-}[514]^{\dagger}$ \\
\hline $4711.1(9)$ & $\leq 2 \mathrm{~ns}$ & $19^{-*}$ & $7 / 2^{-}[514], 9 / 2^{+}[624]$ & $1 / 2^{-}[541], 5 / 2^{+}[402], 7 / 2^{+}[404], 9 / 2^{-}[514]$ \\
\hline $5975.5(13)$ & $\leq 1 \mathrm{~ns}$ & $\left(23^{-}\right)$ & & \\
\hline $6115.2(12)$ & $\sim 1.5 \mathrm{~ns}$ & $\left(23^{-}\right)$ & & \\
\hline $6304.4(13)$ & $\leq 1 \mathrm{~ns}$ & $\left(24^{+}\right)^{*}$ & $7 / 2^{+}[633], 9 / 2^{+}[624]^{\dagger}$ & $5 / 2^{+}[402], 7 / 2^{+}[404], 9 / 2^{-}[514], 11 / 2^{-}[505]^{\dagger}$ \\
\hline \multicolumn{5}{|c|}{${ }^{181} \mathrm{~W}(\mathrm{Z}=74, \mathrm{~N}=107)[? ? ?]$} \\
\hline $1653.0(3)$ & $182(28) \mathrm{ns}$ & $21 / 2^{+*}$ & $9 / 2^{+}[624]$ & $5 / 2^{+}[402], 7 / 2^{+}[404]$ \\
\hline $1744.8(6)$ & $27(3) \mathrm{ns}$ & $23 / 2^{-*}$ & $9 / 2^{+}[624]$ & $5 / 2^{+}[402], 9 / 2^{-}[514]$ \\
\hline $3943.7(8)$ & $23.6(14) \mathrm{ns}$ & $37 / 2^{-}$ & $7 / 2^{-}[503], 7 / 2^{-}[514], 9 / 2^{+}[624]$ & $5 / 2^{+}[402], 9 / 2^{-}[514]$ \\
\hline \multicolumn{5}{|c|}{${ }^{182} \mathrm{~W}(\mathrm{Z}=74, \mathrm{~N}=108)[$ ? ? ? ? ? ? ? ? ] } \\
\hline $1553.2240(10)$ & $1.27(4) \mathrm{ns}$ & $4^{-*}$ & $1 / 2^{-}[510], 9 / 2^{+}[624]$ & \\
\hline $2230.65(14)$ & $1.3(1) \mu \mathrm{s}$ & $10^{+*}$ & $9 / 2^{+}[624], 11 / 2^{+}[615]$ & \\
\hline $3754.89(21)$ & $37(2) \mathrm{ns}$ & $15^{+*}$ & $7 / 2^{-}[503], 9 / 2^{+}[624]$ & $5 / 2^{+}[402], 9 / 2^{-}[514]$ \\
\hline $3893.69(23)$ & $\leq 7 \mathrm{~ns}$ & $16^{+*}$ & $9 / 2^{+}[624], 11 / 2^{+}[615]$ & $5 / 2^{+}[402], 7 / 2^{+}[404]$ \\
\hline $4040.6(3)$ & $20(1) \mathrm{ns}$ & $17^{-*}$ & $9 / 2^{+}[624], 11 / 2^{+}[615]$ & $5 / 2^{+}[402], 9 / 2^{-}[514]$ \\
\hline \multicolumn{5}{|c|}{${ }^{183} \mathrm{~W}(\mathrm{Z}=74, \mathrm{~N}=109)[? ?]$} \\
\hline $1746.55(11)$ & $12.7(20) \mathrm{ns}$ & $19 / 2^{-*}$ & $1 / 2^{-}[510], 9 / 2^{+}[624], 11 / 2^{+}[615]$ & \\
\hline $1900.96(13)$ & $\leq 3.0 \mathrm{~ns}$ & $\left(19 / 2^{+}\right)^{*}$ & $1 / 2^{-}[510], 7 / 2^{-}[514], 11 / 2^{+}[615]^{\dagger}$ & \\
\hline
\end{tabular}




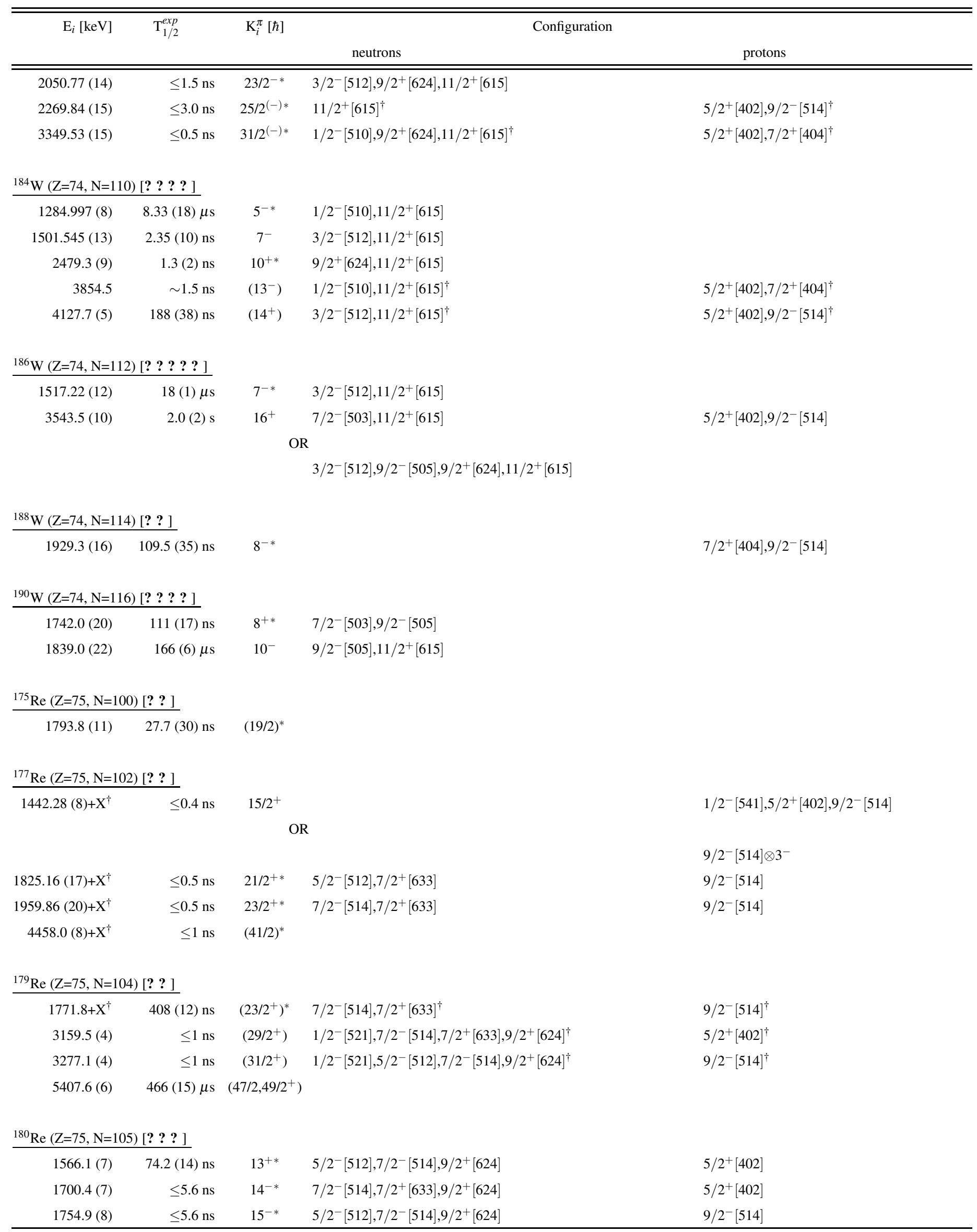




\begin{tabular}{|c|c|c|c|c|}
\hline \multirow[t]{2}{*}{$\mathrm{E}_{i}[\mathrm{keV}]$} & \multirow[t]{2}{*}{$\mathrm{T}_{1 / 2}^{\exp }$} & \multirow[t]{2}{*}{$\mathrm{K}_{i}^{\pi}[\hbar]$} & \multicolumn{2}{|c|}{ Configuration } \\
\hline & & & neutrons & protons \\
\hline $3471.0(8)$ & $9.0(7) \mu \mathrm{s}$ & $21^{-*}$ & $5 / 2^{-}[512], 7 / 2^{-}[514], 9 / 2^{+}[624]$ & $5 / 2^{+}[402], 7 / 2^{+}[404], 9 / 2^{-}[514]$ \\
\hline \multicolumn{5}{|c|}{${ }^{181} \operatorname{Re}(\mathrm{Z}=75, \mathrm{~N}=106)[? ? ? ? ? ? ?]$} \\
\hline $1656.37(14)$ & $250(10) \mathrm{ns}$ & $21 / 2^{-*}$ & $9 / 2^{+}[624], 7 / 2^{-}[514]$ & $5 / 2^{+}[402]$ \\
\hline $1880.57(16)$ & $11.5(9) \mu \mathrm{s}$ & $25 / 2^{+*}$ & $9 / 2^{+}[624], 7 / 2^{-}[514]$ & $9 / 2^{-}[514]$ \\
\hline $3869.40(18)$ & $1.2(2) \mu \mathrm{s}$ & $\left(35 / 2^{-}\right)^{*}$ & $1 / 2^{-}[521], 9 / 2^{+}[624]^{\dagger}$ & $5 / 2^{+}[402], 9 / 2^{-}[514], 11 / 2^{-}[505]^{\dagger}$ \\
\hline $3990.02(17)$ & $22.2(5) \mathrm{ns}$ & $\left(37 / 2^{-}\right)^{*}$ & $9 / 2^{+}[624], 5 / 2^{-}[512], 7 / 2^{+}[633], 7 / 2^{-}[503]^{\dagger}$ & $9 / 2^{-}[514]^{\dagger}$ \\
\hline \multicolumn{5}{|c|}{${ }^{182} \operatorname{Re}(\mathrm{Z}=75, \mathrm{~N}=107)[?$ ? ? ? $]$} \\
\hline $2256.48(19)$ & $82(1) \mathrm{ns}$ & $16^{-*}$ & $7 / 2^{-}[514], 7 / 2^{-}[503], 9 / 2^{+}[624]$ & $9 / 2^{-}[514]$ \\
\hline \multicolumn{5}{|c|}{${ }^{183} \operatorname{Re}(\mathrm{Z}=75, \mathrm{~N}=108)[? ? ? ? ? ?$ ? ? ] } \\
\hline $1907.29(16)$ & $1.04(5) \mathrm{ms}$ & $25 / 2^{+*}$ & $9 / 2^{+}[624], 11 / 2^{+}[615]$ & $5 / 2^{+}[402]$ \\
\hline $1907.29+X^{\dagger}$ & $10(4) \mathrm{ns}$ & $(21 / 2)^{*}$ & & \\
\hline $2737.26(16)$ & $6.0(5) \mathrm{ns}$ & $29 / 2^{-*}$ & $9 / 2^{+}[624], 11 / 2^{+}[615]$ & $9 / 2^{-}[514]$ \\
\hline \multicolumn{5}{|c|}{${ }^{184} \operatorname{Re}(\mathrm{Z}=75, \mathrm{~N}=109)[? ? ?]$} \\
\hline $1543.15(22)$ & $\leq 6 \mathrm{~ns}$ & $\left(12^{-}\right)^{*}$ & $1 / 2^{-}[510], 9 / 2^{+}[624], 11 / 2^{+}[615]^{\dagger}$ & $5 / 2^{+}[402]^{\dagger}$ \\
\hline $1834.0(3)$ & $\leq 5 \mathrm{~ns}$ & $(14)^{*}$ & & \\
\hline $2406.2(4)$ & $\leq 5 \mathrm{~ns}$ & $(16)^{*}$ & & \\
\hline \multicolumn{5}{|c|}{${ }^{185} \operatorname{Re}(\mathrm{Z}=75, \mathrm{~N}=110)[? ? ?$ ? ] } \\
\hline \multirow[t]{3}{*}{$1206.7(3)$} & $1.3(6) \mathrm{ns}$ & $15 / 2^{-}$ & $1 / 2^{-}[510], 9 / 2^{+}[624]$ & $5 / 2^{+}[402]$ \\
\hline & \multicolumn{3}{|c|}{ OR } & \\
\hline & & & $1 / 2^{-}[510], 11 / 2^{+}[615]$ & $5 / 2^{+}[402]$ \\
\hline $1410.9(4)$ & $5.5(14) \mathrm{ns}$ & $19 / 2^{-*}$ & $3 / 2^{-}[512], 11 / 2^{+}[615]$ & $5 / 2^{+}[402]$ \\
\hline $2124.1(4)$ & $200(4) \mathrm{ns}$ & $25 / 2^{+}$ & $9 / 2^{+}[624], 11 / 2^{+}[615]$ & $5 / 2^{+}[402]$ \\
\hline \multicolumn{5}{|c|}{${ }^{187} \operatorname{Re}(\mathrm{Z}=75, \mathrm{~N}=112)[? ?]$} \\
\hline $1474.38(13)$ & $\leq 3 \mathrm{~ns}$ & $\left(19 / 2^{-}\right)$ & $3 / 2^{-}[512], 11 / 2^{+}[615]^{\dagger}$ & $5 / 2^{+}[402]^{\dagger}$ \\
\hline $1681.68(16)$ & $114(23) \mathrm{ns}$ & $\left(19 / 2^{+}\right)$ & $1 / 2^{-}[510], 11 / 2^{+}[615]^{\dagger}$ & $9 / 2^{-}[514]^{\dagger}$ \\
\hline \multicolumn{5}{|c|}{${ }^{180} \mathrm{Os}(\mathrm{Z}=76, \mathrm{~N}=104)[? ? ? ? ? ?]$} \\
\hline $1928.62(19)$ & $24.9(20) \mathrm{ns}$ & $7^{-*}$ & $7 / 2^{-}[514], 7 / 2^{+}[633]$ & \\
\hline $5848^{\dagger}$ & $0.8(2) \mu \mathrm{s}$ & & & \\
\hline $7592^{\dagger}$ & $72(6) \mathrm{ns}$ & & & \\
\hline \multicolumn{5}{|c|}{${ }^{181} \mathrm{Os}(\mathrm{Z}=76, \mathrm{~N}=105)[? ? ?]$} \\
\hline $1743.3(4)$ & $7(2) \mathrm{ns}$ & $21 / 2^{+*}$ & $5 / 2^{-}[512], 7 / 2^{-}[514], 9 / 2^{+}[624]$ & \\
\hline $3578.3(3)$ & $\leq 5 \mathrm{~ns}$ & $33 / 2^{-}$ & $1 / 2^{-}[521], 7 / 2^{-}[514], 9 / 2^{+}[624]$ & $5 / 2^{+}[402], 11 / 2^{-}[505]$ \\
\hline $3737.7(3)$ & $24(4) \mathrm{ns}$ & $35 / 2^{-}$ & $1 / 2^{-}[521], 7 / 2^{-}[514], 9 / 2^{+}[624]$ & $7 / 2^{+}[404], 11 / 2^{-}[505]$ \\
\hline \multicolumn{5}{|c|}{${ }^{182} \mathrm{Os}(\mathrm{Z}=76, \mathrm{~N}=106)[? ? ? ? ? ?$ ? $]$} \\
\hline $1831.4(3)$ & $0.78(7) \mathrm{ms}$ & $8^{-*}$ & $7 / 2^{-}[514], 9 / 2^{+}[624]$ & \\
\hline $4370.3(5)$ & $6(2) \mathrm{ns}$ & $16^{(+) *}$ & $7 / 2^{-}[514], 9 / 2^{+}[624]^{\dagger}$ & $5 / 2^{+}[402], 11 / 2^{-}[505]^{\dagger}$ \\
\hline
\end{tabular}




\begin{tabular}{|c|c|c|c|c|}
\hline \multirow[t]{2}{*}{$\mathrm{E}_{i}[\mathrm{keV}]$} & \multirow[t]{2}{*}{$\mathrm{T}_{1 / 2}^{\exp }$} & \multirow[t]{2}{*}{$\mathrm{K}_{i}^{\pi}[\hbar]$} & \multicolumn{2}{|l|}{ Configuration } \\
\hline & & & neutrons & protons \\
\hline $7049.5(4)$ & $146(9) \mathrm{ns}$ & $25^{+*}$ & $7 / 2^{-}[514], 7 / 2^{-}[503], 7 / 2^{+}[633], 9 / 2^{+}[624]$ & $9 / 2^{-}[514], 11 / 2^{-}[505]$ \\
\hline \multicolumn{5}{|c|}{${ }^{183} \mathrm{Os}(\mathrm{Z}=76, \mathrm{~N}=107)[? ? ?]$} \\
\hline $1560.26(9)$ & $\leq 3 \mathrm{~ns}$ & $15 / 2^{-*}$ & $9 / 2^{+}[624]$ & $1 / 2^{-}[541], 5 / 2^{+}[402]$ \\
\hline $2209.62(10)$ & $\leq 3 \mathrm{~ns}$ & $23 / 2^{+}$ & $7 / 2^{-}[503], 7 / 2^{-}[514], 9 / 2^{+}[624]$ & \\
\hline $3785.51(12)$ & $\leq 3 \mathrm{~ns}$ & $33 / 2^{+*}$ & $7 / 2^{-}[503], 7 / 2^{-}[514], 9 / 2^{+}[624]$ & $1 / 2^{-}[541], 9 / 2^{-}[514]$ \\
\hline $5067.54(15)$ & $27(3) \mathrm{ns}$ & $43 / 2^{(-) *}$ & $1 / 2^{-}[512], 5 / 2^{-}[512], 7 / 2^{-}[503], 7 / 2^{-}[514], 9 / 2^{+}[624]^{\dagger}$ & $5 / 2^{+}[402], 9 / 2^{-}[514]^{\dagger}$ \\
\hline $5167.43(15)$ & $24(2) \mathrm{ns}$ & $43 / 2^{+*}$ & $7 / 2^{-}[503], 7 / 2^{-}[514], 9 / 2^{+}[624]$ & $1 / 2^{-}[541], 5 / 2^{+}[402], 7 / 2^{+}[404], 9 / 2^{-}[514]$ \\
\hline \multicolumn{5}{|c|}{${ }^{184} \mathrm{Os}(\mathrm{Z}=76, \mathrm{~N}=108)[? ? ? ? ?]$} \\
\hline $2046.71(19)$ & $\leq 1.4 \mathrm{~ns}$ & $8^{-*}$ & $7 / 2^{-}[503], 9 / 2^{+}[624]$ & \\
\hline $2366.81(19)$ & $23.6(14) \mathrm{ns}$ & $10^{+*}$ & $9 / 2^{+}[624], 11 / 2^{+}[615]$ & \\
\hline $4756.71(24)$ & $48(5) \mathrm{ns}$ & $\left(18^{-}\right)$ & $9 / 2^{+}[624], 11 / 2^{+}[615]^{\dagger}$ & $5 / 2^{+}[402], 11 / 2^{-}[505]^{\dagger}$ \\
\hline $5565.7(3)$ & $\leq 1.4 \mathrm{~ns}$ & $\left(20^{+}\right)$ & $9 / 2^{+}[624], 11 / 2^{+}[615]^{\dagger}$ & $9 / 2^{-}[514], 11 / 2^{-}[505]^{\dagger}$ \\
\hline $5743.3(4)$ & $1.04(21) \mathrm{ns}$ & $\left(21^{+}\right)$ & $7 / 2^{-}[503], 7 / 2^{-}[514], 9 / 2^{+}[624], 11 / 2^{+}[615]^{\dagger}$ & $3 / 2^{+}[402], 5 / 2^{+}[402]^{\dagger}$ \\
\hline $6186.5(4)$ & $0.35(14) \mathrm{ns}$ & $\left(22^{+}\right)$ & $7 / 2^{-}[503], 7 / 2^{-}[514], 9 / 2^{+}[624], 11 / 2^{+}[615]^{\dagger}$ & $1 / 2^{-}[541], 9 / 2^{-}[514]^{\dagger}$ \\
\hline $6598.7(4)$ & $0.42(14) \mathrm{ns}$ & $\left(23^{+}\right)$ & $3 / 2^{-}[512], 7 / 2^{+}[633], 9 / 2^{+}[624], 11 / 2^{+}[615]^{\dagger}$ & $5 / 2^{+}[402], 11 / 2^{-}[505]^{\dagger}$ \\
\hline $7311.1(4)$ & $0.90(21) \mathrm{ns}$ & $\left(25^{+}\right)$ & $3 / 2^{-}[512], 7 / 2^{-}[514], 9 / 2^{+}[624], 11 / 2^{+}[615]^{\dagger}$ & $9 / 2^{-}[514], 11 / 2^{-}[505]^{\dagger}$ \\
\hline \multicolumn{5}{|c|}{${ }^{185} \mathrm{Os}(\mathrm{Z}=76, \mathrm{~N}=109)[? ? ? ?]$} \\
\hline $1987.13(11)$ & $5.5(10) \mathrm{ns}$ & $23 / 2^{-*}$ & $3 / 2^{-}[512], 9 / 2^{+}[624], 11 / 2^{+}[615]$ & \\
\hline $5007.1(3)$ & $18(2) \mathrm{ns}$ & $(41 / 2)$ & & \\
\hline \multicolumn{5}{|c|}{${ }^{186} \mathrm{Os}(\mathrm{Z}=76, \mathrm{~N}=110)[? ? ? ? ? ? ? \mathrm{e}]$} \\
\hline $1628.54(11)$ & $\leq 1 \mathrm{~ns}$ & $5^{-*}$ & $1 / 2^{-}[510], 11 / 2^{+}[615]$ & \\
\hline $1774.65(21)$ & $8.36(24) \mathrm{ns}$ & $7^{-*}$ & $3 / 2^{-}[512], 11 / 2^{+}[615]$ & \\
\hline $2165.5(3)$ & $5.7(4) \mathrm{ns}$ & $9^{-*}$ & $7 / 2^{-}[503], 11 / 2^{+}[615]$ & \\
\hline $2562.7(3)$ & $\leq 1 \mathrm{~ns}$ & $\left(10^{+}\right)^{*}$ & $9 / 2^{+}[624], 11 / 2^{+}[615]^{\dagger}$ & \\
\hline $4493.69(23)$ & $\leq 0.5 \mathrm{~ns}$ & $\left(18^{+}\right)$ & $7 / 2^{-}[503], 9 / 2^{-}[505], 9 / 2^{+}[624], 11 / 2^{+}[615]^{\dagger}$ & \\
\hline $5024.70(25)$ & $\leq 2 \mathrm{~ns}$ & $\left(18^{-}\right)^{*}$ & $9 / 2^{+}[624], 11 / 2^{+}[615]^{\dagger}$ & $5 / 2^{+}[402], 11 / 2^{-}[505]^{\dagger}$ \\
\hline $5330.9(3)$ & $\leq 1 \mathrm{~ns}$ & $\left(19^{-}\right)^{*}$ & $7 / 2^{-}[503], 11 / 2^{+}[615]^{\dagger}$ & $9 / 2^{-}[514], 11 / 2^{-}[505]^{\dagger}$ \\
\hline $7141.9(17)$ & $\leq 2 \mathrm{~ns}$ & $\left(28^{+}\right)$ & $7 / 2^{-}[503], 9 / 2^{-}[505], 9 / 2^{+}[624], 11 / 2^{+}[615]^{\dagger}$ & $9 / 2^{-}[514], 11 / 2^{-}[505]^{\dagger}$ \\
\hline \multicolumn{5}{|c|}{${ }^{188} \mathrm{Os}(\mathrm{Z}=76, \mathrm{~N}=112)[? ? ?]$} \\
\hline $1771.0(5)$ & $14.0(10) \mathrm{ns}$ & $7^{-*}$ & $3 / 2^{-}[512], 11 / 2^{+}[615]$ & \\
\hline $2144.8(5)$ & $12.3(2) \mathrm{ns}$ & $10^{-*}$ & $9 / 2^{-}[505], 11 / 2^{+}[615]$ & \\
\hline \multicolumn{5}{|c|}{${ }^{190} \mathrm{Os}(\mathrm{Z}=76, \mathrm{~N}=114)[? ? ? ? ? ?]$} \\
\hline $1705.4(2)$ & $9.9(1) \mathrm{m}$ & $10^{-}$ & $9 / 2^{-}[505], 11 / 2^{+}[615]$ & \\
\hline \multicolumn{5}{|c|}{${ }^{191}$ Os $(\mathrm{Z}=76, \mathrm{~N}=115)[? ? ? ? ? ?]$} \\
\hline $1424.5(4)$ & $16.6(7) \mathrm{ns}$ & $23 / 2^{+}$ & $3 / 2^{-}[512], 9 / 2^{-}[505], 11 / 2^{+}[615]$ & \\
\hline $2640.4(4)$ & $17.7(4) \mathrm{ns}$ & $31 / 2^{+*}$ & $11 / 2^{+}[615]$ & $9 / 2^{-}[514], 11 / 2^{-}[505]$ \\
\hline${ }^{192} \mathrm{Os}(\mathrm{Z}=76, \mathrm{~N}=11$ & [? ? ? ? ? ? & & & \\
\hline
\end{tabular}




\begin{tabular}{|c|c|c|c|c|}
\hline \multirow[t]{2}{*}{$\mathrm{E}_{i}[\mathrm{keV}]$} & \multirow[t]{2}{*}{$\mathrm{T}_{1 / 2}^{\exp }$} & \multirow[t]{2}{*}{$\mathrm{K}_{i}^{\pi}[\hbar]$} & \multicolumn{2}{|r|}{ Configuration } \\
\hline & & & neutrons & protons \\
\hline $2015.39(10)$ & $5.9(1) \mathrm{s}$ & $10^{-*}$ & $9 / 2^{-}[505], 11 / 2^{+}[615]$ & \\
\hline $2864.4(4)$ & $1.4(3) \mathrm{ns}$ & $\mathrm{I}^{\pi}=12^{+*}$ & & \\
\hline $4580.1(11)$ & $205(7) \mathrm{ns}$ & $\mathrm{I}^{\pi}=20^{+*}$ & & \\
\hline \multicolumn{5}{|c|}{${ }^{181} \operatorname{Ir}(\mathrm{Z}=77, \mathrm{~N}=104)[? ? ?]$} \\
\hline $2034.3(4)$ & $22(4) \mathrm{ns}$ & $23 / 2^{+*}$ & $7 / 2^{+}[633], 7 / 2^{-}[514]$ & $9 / 2^{-}[514]$ \\
\hline \multicolumn{5}{|c|}{${ }^{184} \mathrm{Pt}(\mathrm{Z}=78, \mathrm{~N}=106)[? ? ?]$} \\
\hline $1842.5(8)$ & $1.01(5) \mathrm{ms}$ & $8^{-*}$ & $7 / 2^{-}[514], 9 / 2^{+}[624]$ & \\
\hline \multicolumn{5}{|c|}{${ }^{186} \mathrm{Pt}(\mathrm{Z}=78, \mathrm{~N}=108)[? ? ? ?]$} \\
\hline $2195.6(6)$ & $8.0(13) \mathrm{ns}$ & $\left(8^{-}\right)^{*}$ & $7 / 2^{-}[514], 9 / 2^{+}[624]$ & \\
\hline \multicolumn{5}{|c|}{${ }^{188} \mathrm{Pt}(\mathrm{Z}=78, \mathrm{~N}=110)[? ?]$} \\
\hline $1768.53(17)$ & $0.20(2) \mathrm{ns}$ & $\mathrm{I}^{\pi}=7^{-}$ & & \\
\hline $2811.0(3)$ & $0.66(4) \mathrm{ns}$ & $\mathrm{I}^{\pi}=12^{+}$ & & \\
\hline \multicolumn{5}{|c|}{${ }^{190} \mathrm{Pt}(\mathrm{Z}=78, \mathrm{~N}=112)[$ ? ? ? ? ? ? ? ] } \\
\hline $1631.1(1)$ & $0.79(5) \mathrm{ns}$ & $\mathrm{I}^{\pi}=7^{-}$ & & \\
\hline $2297.4(3)$ & $48(4) \mathrm{ns}$ & $10^{-}$ & $9 / 2^{-}[505], 11 / 2^{+}[615]$ & \\
\hline $2726.7(3)$ & $1.39(12) \mathrm{ns}$ & $\mathrm{I}^{\pi}=12^{+}$ & & \\
\hline \multicolumn{5}{|c|}{${ }^{192} \mathrm{Pt}(\mathrm{Z}=78, \mathrm{~N}=114)[? ? ? ? ? ? ? ? ? ?$ ? $]$} \\
\hline $1518.35(8)$ & $1.85(17) \mathrm{ns}$ & $\mathrm{I}^{\pi}=7^{-}$ & & \\
\hline $2172.37(13)$ & $272(23) \mathrm{ns}$ & $10^{-}$ & $9 / 2^{-}[505], 11 / 2^{+}[615]$ & \\
\hline $2623.72(18)$ & $2.62(18) \mathrm{ns}$ & $\mathrm{I}^{\pi}=12^{+}$ & & \\
\hline \multicolumn{5}{|c|}{${ }^{186} \mathrm{Hg}(\mathrm{Z}=80, \mathrm{~N}=106)[? ? ? ? ?$ ? $]$} \\
\hline $2217.1(5)$ & $82(5) \mu \mathrm{s}$ & $8^{-*}$ & $7 / 2^{-}[514], 9 / 2^{+}[624]$ & \\
\hline \multicolumn{5}{|c|}{${ }^{188} \mathrm{~Pb}(\mathrm{Z}=82, \mathrm{~N}=106)[? ? ?]$} \\
\hline $2577.3(3)$ & $800(20) \mathrm{ns}$ & $8^{-*}$ & $7 / 2^{-}[514], 9 / 2^{+}[624]$ & \\
\hline \multicolumn{5}{|c|}{${ }^{234} \mathrm{U}(\mathrm{Z}=92, \mathrm{~N}=142)[?$ ? ? ? ? ] } \\
\hline $1421.32(10)$ & $33.5(20) \mu \mathrm{s}$ & $6^{-}$ & $5 / 2^{+}[633], 7 / 2^{-}[743]$ & \\
\hline $1552.62(10)$ & $2.20(25) \mathrm{ns}$ & $5^{+}$ & $5 / 2^{+}[633], 5 / 2^{+}[622]$ & \\
\hline \multicolumn{5}{|c|}{${ }^{236} \mathrm{U}(\mathrm{Z}=92, \mathrm{~N}=144)[?$ ? ? ? ] } \\
\hline $1052.89(19)$ & $102(4) \mathrm{ns}$ & $4^{-}$ & $1 / 2^{+}[631], 7 / 2^{-}[743]$ & \\
\hline \multicolumn{5}{|c|}{${ }^{238} \mathrm{Pu}(\mathrm{Z}=94, \mathrm{~N}=144)[? ? ?]$} \\
\hline $1082.56(6)$ & $8.5(5) \mathrm{ns}$ & $4^{-}$ & $1 / 2^{+}[631], 7 / 2^{-}[743]$ & \\
\hline \multicolumn{5}{|c|}{${ }^{240} \mathrm{Pu}(\mathrm{Z}=94, \mathrm{~N}=146)[? ? ?]$} \\
\hline $1030.53(5)$ & $1.32(15) \mathrm{ns}$ & $3^{+}$ & $1 / 2^{+}[631], 5 / 2^{+}[622]$ & \\
\hline
\end{tabular}




\begin{tabular}{ccccc}
\hline \hline $\mathrm{E}_{i}[\mathrm{keV}]$ & $\mathrm{T}_{1 / 2}^{\text {exp }}$ & $\mathrm{K}_{i}^{\pi}[\hbar]$ & Configuration & \\
& & neutrons & protons \\
\hline \hline
\end{tabular}

$1308.74(5) \quad 165(10) \mathrm{ns} \quad 5^{-} \quad 5 / 2^{-}[523], 5 / 2^{+}[622]$

${ }^{244} \mathrm{Pu}(\mathrm{Z}=94, \mathrm{~N}=150)[? ? ?$ ? $]$

$1216.0(5) \quad 1.75(12) \mathrm{s} \quad\left(8^{-}\right) \quad 7 / 2^{+}[624], 9 / 2^{-}[734]^{\dagger}$

${ }^{244} \mathrm{Cm}(\mathrm{Z}=96, \mathrm{~N}=148)[$ ? ? ? ? ? ]

$1040.188(12) \quad 34(2) \mathrm{ms} \quad 6^{+} \quad 5 / 2^{+}[622], 7 / 2^{+}[624]$

${ }^{246} \mathrm{Cm}(\mathrm{Z}=96, \mathrm{~N}=150)[$ ? ? ? ? ? ? ? ]

$1179.66(13) \quad 1.12(24) \mathrm{s} \quad 8^{-} \quad 7 / 2^{+}[624], 9 / 2^{-}[734]$

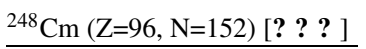

$1458.1(10) \quad 146(18) \mu \mathrm{s} \quad\left(8^{-}\right) \quad 7 / 2^{+}[633], 9 / 2^{-}[734]^{\dagger}$ or

$7 / 2^{-}[514], 9 / 2^{+}[624]^{\dagger}$

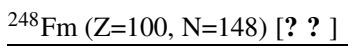
$1188^{\dagger}$
$10.1(6) \mathrm{ms}$
$\left(6^{+}\right) \quad 5 / 2^{+}[622], 7 / 2^{+}[624]^{\dagger}$

${ }^{250} \mathrm{Fm}(\mathrm{Z}=100, \mathrm{~N}=150)[$ ? ? ? ? ? ? ? ]

$1199.3(15) \quad 1.92(5) \mathrm{s} \quad 8^{-*} \quad 7 / 2^{+}[624], 9 / 2^{-}[734]$

$1199.3(15)+\mathrm{X}^{\dagger} \quad 8(2) \mu \mathrm{s}$

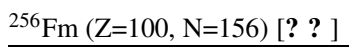

$1425.3(4) \quad 70(5) \mathrm{ns} \quad 7^{-}$

$7 / 2^{-}[514], 7 / 2^{+}[633]$

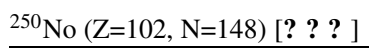

$800+\mathrm{X}^{\dagger} \quad 54(10) \mu \mathrm{s} \quad\left(6^{+}\right) \quad 5 / 2^{+}[622], 7 / 2^{+}[624]^{\dagger}$

$\frac{{ }^{252} \mathrm{No}(\mathrm{Z}=102, \mathrm{~N}=150)[? ? ? ? \text { ? ? ? }]}{1254.7(15)} \quad 8^{-*} \quad 7 / 2^{+}[624], 9 / 2^{-}[734]$

$\stackrel{{ }^{253} \mathrm{No}(\mathrm{Z}=102, \mathrm{~N}=151)[? ? ? ?]}{ }$

$1128.2+X^{\dagger} \quad 706(24) \mu \mathrm{s} \quad\left(25 / 2^{+}\right) \quad 9 / 2^{-}[734]^{\dagger} \quad 7 / 2^{-}[514], 9 / 2^{+}[624]^{\dagger}$

${ }^{254}$ No $(\mathrm{Z}=102, \mathrm{~N}=152)[$ ? ? ? ? ? ? ? ? ? ? ]

\begin{tabular}{|c|c|c|c|c|}
\hline $1294.9(12)$ & $265.0(14) \mathrm{ms}$ & $8^{-*}$ & & $7 / 2^{-}[514], 9 / 2^{+}[624]$ \\
\hline $2918.1(17)^{\dagger}$ & $183.8(16) \mu \mathrm{s}$ & $\left(16^{+}\right)$ & $7 / 2^{+}[624], 9 / 2^{-}[734]^{\dagger}$ & $7 / 2^{-}[514], 9 / 2^{+}[624]^{\dagger}$ \\
\hline
\end{tabular}

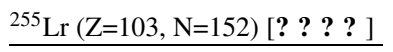

$740.0+\mathrm{Y}^{\dagger} \quad 1.76(6) \mathrm{ms}$

${ }^{256} \mathrm{Rf}(\mathrm{Z}=104, \mathrm{~N}=152)$ [? ? ? ? ]

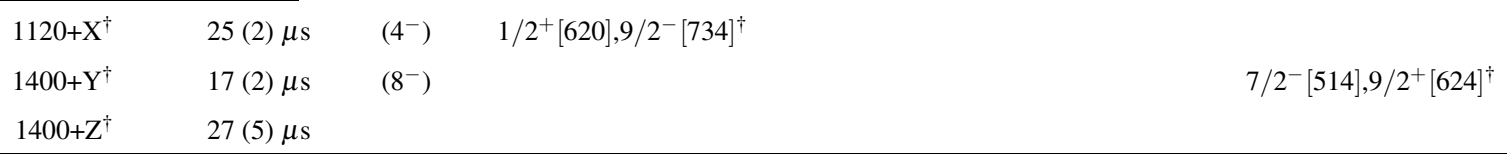




\begin{tabular}{ccccc}
\hline \hline $\mathrm{E}_{i}[\mathrm{keV}]$ & $\mathrm{T}_{1 / 2}^{\exp }$ & $\mathrm{K}_{i}^{\pi}[\hbar]$ & Configuration & protons \\
& & neutrons & \\
\hline \hline
\end{tabular}

${ }^{257} \operatorname{Rf}(\mathrm{Z}=104, \mathrm{~N}=153)$ [? ? ? ? ? ?]

$1083.2(17) \quad 106(6) \mu \mathrm{s} \quad\left(21 / 2^{+}\right) \quad 11 / 2^{-}[725]^{\dagger} \quad 1 / 2^{-}[521], 9 / 2^{+}[624]^{\dagger}$

${ }^{262} \operatorname{Rf}(\mathrm{Z}=104, \mathrm{~N}=158)$ [? ? ]

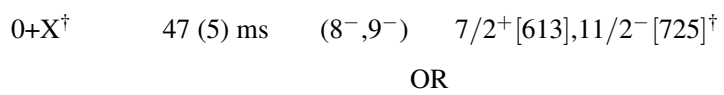

$7 / 2^{-}[514], 9 / 2^{+}[624]^{\dagger}$

${ }^{266} \mathrm{Hs}(\mathrm{Z}=108, \mathrm{~N}=158)[? ?]$
1404 (97) 74 (+354-34) ms
$\left(9^{-}\right) \quad 7 / 2^{+}[613], 11 / 2^{-}[725]^{\dagger}$

${ }^{270}$ Ds $(\mathrm{Z}=110, \mathrm{~N}=160)[? ? ?]$

$1348(66) \quad 3.9(+13-8) \mathrm{ms} \quad\left(10^{-}\right) \quad 9 / 2^{+}[615], 11 / 2^{-}[725]^{\dagger}$ 